\title{
Resource Allocation in Cognitive Radio Networks
}

by

\author{
Athipat Limmanee \\ Submitted in total fulfilment of \\ the requirements for the degree of
}

Doctor of Philosophy

Department of Electrical and Electronic Engineering

The University of Melbourne

Australia

September, 2012

Produced on acid-free paper 
The University of Melbourne

Australia

\section{Abstract \\ Resource Allocation in Cognitive Radio Networks}

by Athipat Limmanee

This thesis focuses on optimal power allocation problems for various types of spectrum-sharing based cognitive radio networks in the presence of delay-sensitive primary links. To guarantee the quality of service in the delay-sensitive primary network, primary user's outage probability constraint (POC) is imposed such that the transmission outage probability of each primary user is confined under the predefined threshold.

We first consider a cognitive radio network consisting of a secondary user (SU) equipped with orthogonal frequency-division multiplexing (OFDM) technology able to access $N$ randomly fading frequency bands for transmitting delay-insensitive as well as delay-sensitive traffic. Each band is licensed to an individual single-antenna and delay-sensitive primary user (PU) whose quality of service is assured by a POC. Assuming full channel state information (CSI) is available at the secondary network, we solve the SU's ergodic capacity maximization problem subject to SU's average transmit power, SU's outage probability constraints (SOC) and all POCs by using a rigorous probabilistic power allocation technique. A suboptimal power control policy is also proposed to reduce the high computational complexity when $N$ is large.

Next, we study cognitive broadcast channels with a single-antenna secondary base station (SBS) and $M$ single-antenna secondary receivers (SRs) sharing the same spectrum band with one single-antenna and delay-sensitive PU. The SBS aims to maximize the ergodic sum downlink throughput to all $M$ SRs subject to a POC and a transmit power constraint at the SBS. With full CSI available at the secondary network, the optimal solution reveals that at each timeslot SBS will choose the SR with the highest direct channel power gain and allocate the timeslot to that user. The 
opportunistic scheduling aspect from the optimality condition allows us to further analyze the downlink throughput scaling behavior in Rayleigh fading channel as $M$ grows large.

We then examine a cognitive multiple-access channels with a single-antenna SBS and $M$ single-antenna secondary transmitters sharing the same spectrum band with a single-antenna and delay-sensitive PU. Under an average transmit power constraint in each secondary transmitters and a POC at the primary link, we characterize the ergodic capacity region and two outage capacity regions, i.e. common outage capacity region and individual outage capacity region, in the secondary uplink network by exploiting the polymatroid structure of the problems. Also, the derivation of the associated optimal power allocation schemes are provided. The optimal solutions for the problems demonstrate that successive decoding is optimal and the decoding order can be solved explicitly as a function of joint channel state.

Finally, we investigate a transmit power allocation problem for minimizing outage probability of a single-antenna SU subject to a POC at a delay-sensitive and singleantenna PU and an average transmit power constraint at the SU, providing that the SU has quantized channel side information via $B$-bit feedback from the band manager. By using nearest neighbourhood condition, we can derive the optimal channel partition structure for the vector channel space, making Karush-Kuhn-Tucker condition applicable as a necessary condition for finding a locally optimal solution. We also propose another low-complexity suboptimal algorithm. Numerical results show that the SU's outage probability performance from the suboptimal algorithm approaches the SU's outage probability performance in the locally-optimal algorithm as the number of feedback bits, $B$, increases. Besides, we include the asymptotic analysis on the SU's outage probability when $B$ is large. 
This is to certify that

(i) the thesis comprises only my original work,

(ii) due acknowledgment has been made in the text to all other material used,

(iii) the thesis is less than 100,000 words in length, exclusive of table, maps, bibliographies, appendices and footnotes.

Signature

Date 


\section{Acknowledgments}

Foremost, I would like to thank my supervisor Professor Subhrakanti Dey for his continuing guidance, encouragement and support throughout my PhD study. I have benefited greatly from his technical insights. In addition, I am indebted Dr. Randa Zakhour for her insightful and careful way of looking at problems, leading to the result in Chapter 5. I wish to thank my lab colleagues, including Ehsan Nekouei, Wang Chih-Hong, Paul Tune, Hsu Chih-yu and Guo Xiaoxi for their friendship. Especially, Chapter 3 was the result of joint work with Ehsan.

My gratitude goes to all people who support me through PhD study in Melbourne. I wish to thank Panit Terdsudthironapoom who had spent his effort to find my first accommodation before I arrived Melbourne. My thanks also go to Hoey ${ }^{+}$ and $\mathrm{P}^{\prime}$ Mae for being my peculiarly inspiring friends.

Further, I wish to express my thankfulness to all who have shaped and supported me throughout my life: my teachers and my friends at Suankularb Wittayalai School particularly Ajarn Pornthip Hiranburana and Ajarn Malee Tantikul; my teachers at SIIT especially Associate Professor Chalie Charoenlarpnopparut and Associate Professor Waree Kongprawechnon for their support when I was searching for a graduate scholarship; The Vipatakanok family - in particular: Mrs. Sa-ingmas, Mr. Nipon and Ms. Supawan, whom I spent some part of my childhood with; my friends at SIIT especially Opart Sanmontrikul whom I owe a great deal for his friendship; Thanachai Leelanuchakul and Warongporn Pongpinyopap for being my very good friends for more than a decade.

Last but not least, I would like to thank my parents for their love, understanding and patience. 


\section{List of Publications}

\section{Conference Papers}

1. A. Limmanee, S. Dey, and J.S. Evans. "Service-outage Capacity Maximization in Cognitive Radio". In ICC'2011, Kyoto, Japan, June 2011.

2. A. Limmanee and S. Dey. "Optimal Power Policy and Throughput Analysis in Cognitive Broadcast Channel under Primary's Outage Constraint". In RAWNET'2012, Paderborn, Germany, May 2012.

\section{Journal Papers}

1. A. Limmanee, S. Dey, and J.S. Evans. "Service-outage Capacity Maximization in Cognitive Radio for Parallel Fading Channels". IEEE Trans. Communications (In press).

2. A. Limmanee, S. Dey, and E. Nekouei. "Optimal Power Policies and Throughput Scaling Analyses in Fading Cognitive Broadcast Channels with Primary Outage Probability Constraint". IEEE Trans. Communications (Submitted).

3. A. Limmanee and S. Dey. "Optimal Power Allocation Policies in Fading Cognitive Multiple-access Channels with A Primary Outage Constraint" (In preparation).

4. A. Limmanee, R. Zakhour, and S. Dey. "Outage Minimization under Primary Outage Constraint with Quantized Feedback in Cognitive Radio" (In preparation). 


\section{Contents}

1 Introduction $\quad 1$

1.1 Motivation . . . . . . . . . . . . . . . . . . 1

1.2 Literature review . . . . . . . . . . . . . . . . . . . 3

1.2.1 Capacity in wireless channel . . . . . . . . . . . . . . 4

1.2.2 Cognitive radio network paradigms . . . . . . . . . . 8

1.3 Outline and contribution of the thesis . . . . . . . . . . . . 15

2 Service-outage Capacity Maximization in Cognitive Radio for Par$\begin{array}{ll}\text { allel Fading Channels } & 21\end{array}$

2.1 System model . . . . . . . . . . . . . . . . . . . . . . . . . . . 24

2.2 Problem formulation . . . . . . . . . . . . . . . . . . . 26

2.3 Main results . . . . . . . . . . . . . . . . . . . . . . . 29

2.3.1 Feasibility of the service-outage problem . . . . . . . . . 33

2.3.2 Proposed suboptimal power control . . . . . . . . . . . . 36

2.4 Numerical results . . . . . . . . . . . . . . . . . . . . . . . . . . 38

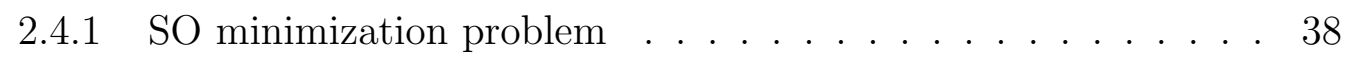

2.4.2 SEC maximization problem . . . . . . . . . . . . . . 40

2.4.3 Suboptimal power allocation scheme . . . . . . . . . . . 44

2.5 Conclusion . . . . . . . . . . . . . . . . . . . . . . . . 46

3 Power Allocation in Cognitive Broadcast Channels with Primary $\begin{array}{ll}\text { Outage Probability Constraint } & 47\end{array}$

3.1 System model . . . . . . . . . . . . . . . . . . . 50

3.2 Optimal power strategies . . . . . . . . . . . . . . . . 53

3.2.1 Optimal power policy for ATPC . . . . . . . . . . . 54

3.2.2 Optimal power policy for PTPC . . . . . . . . . . . 55

3.3 SU throughput scaling with $\mathrm{ON}-\mathrm{OFF}$ power policy at PU . . . . . . . 56

3.3.1 Throughput scaling in ATPC case . . . . . . . . . . . . 58

3.3.2 Throughput scaling in PTPC case . . . . . . . . . . . . 60

3.4 SU throughput scaling with TCI power policy at PU . . . . . . . . 63

3.4.1 Throughput scaling in ATPC case . . . . . . . . . . . 65

3.4.2 Throughput scaling in PTPC case . . . . . . . . . . . . 66

3.5 Numerical results . . . . . . . . . . . . . . . . . . . . . . . . . . 67

3.5.1 The effect of POC on sum ergodic capacity in C-BC channel . 67

3.5.2 Throughput scaling results in the secondary network . . . . 68

3.6 Conclusion . . . . . . . . . . . . . . . . . . . . . . . . 73

4 Power Allocation in Cognitive Multiple-access Channels with Pri$\begin{array}{ll}\text { mary Outage Probability Constraint } & 75\end{array}$

4.1 System model . . . . . . . . . . . . . . . . . . 78 
4.2 Ergodic capacity region . . . . . . . . . . . . . . . 80

4.2.1 Special case: Ergodic sum rate maximization problem with ATPC and POC .................. . . 81

4.2.2 General case: Ergodic Capacity Region for Secondary Network with ATPC and POC . . . . . . . . . . . . . 85

4.2 .3 Discussion . . . . . . . . . . . . . . . . . . . . . . . . . . . . . . . 92

4.3 Outage capacity region . . . . . . . . . . . . . . . . . 94

4.3.1 Definition of common outage capacity and individual outage

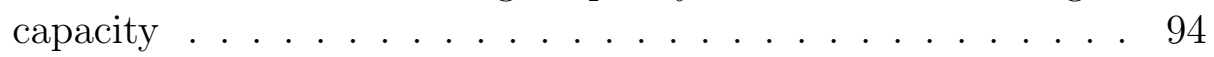

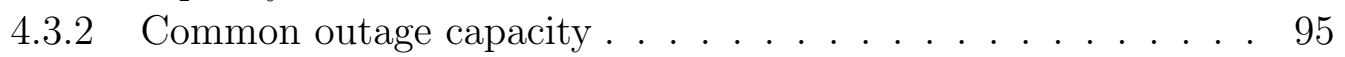

4.3.3 Individual outage capacity . . . . . . . . . . . . . . . . . 101

4.4 Simulation results . . . . . . . . . . . . . . . . . . . . . . . . 108

4.4.1 Ergodic capacity results . . . . . . . . . . . . . . . 108

4.4 .2 Outage probability results . . . . . . . . . . . . 110

4.5 Conclusions . . . . . . . . . . . . . . . . . . . . . . . . 114

5 Outage Minimization under Primary Outage Constraint with Quan$\begin{array}{ll}\text { tized Feedback } & 117\end{array}$

5.1 Problem statement and system model . . . . . . . . . . . . . . 120

5.2 SU outage minimization problem with quantized feedback . . . . . . 121

5.2.1 Optimal channel partition structure and searching algorithm . 123

5.2.2 Locally optimal power codebook from KKT condition . . . . . 125

5.2.3 Proposed suboptimal algorithm . . . . . . . . . . . . . 126

5.3 Asymptotic analysis on SU outage probability for ZFLP . . . . . . . 128

5.4 Simulation results . . . . . . . . . . . . . . . . . . . . 130

5.5 Conclusion . . . . . . . . . . . . . . . . . . . 134

6 Conclusions 137

6.1 Summary . . . . . . . . . . . . . . . . . . . 137

6.2 Future research . . . . . . . . . . . . . . . . . . . . 139

$\begin{array}{lr}\text { A Proofs in Chapter 2 } & 141\end{array}$

A.1 Proof of Lemma 2.2.1 . . . . . . . . . . . . . . . . . . . 141

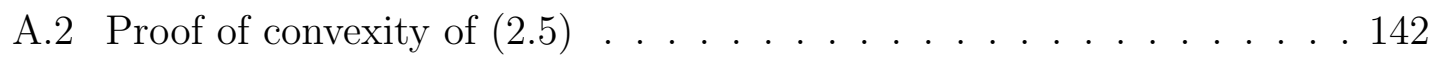

A.3 Proof of Theorem 2.3.1 . . . . . . . . . . . . . . . . 143

B Proofs in Chapter 3 153

B.1 Proof of Lemma 3.2.1 f . . . . . . . . . . . . . . . 153

B.2 KKT conditions for ATPC . . . . . . . . . . . . . . . . . . . . . . 154

B.3 Proof of Theorem 3.2.1 . . . . . . . . . . . . . . . . . . 154

B.4 KKT conditions for PTPC . . . . . . . . . . . . 155

B.5 Proof of Theorem $3.2 .2 \ldots \ldots \ldots \ldots$

B.6 Proof of Lemma 3.3.1 . . . . . . . . . . . . . . . . . 156

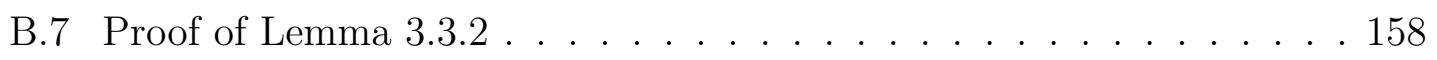

B.8 Lower bound on $k_{o}$ when $\epsilon_{p}>\epsilon_{p}^{0}$ in ATPC with ON-OFF power control at the primary user . . . . . . . . . . . . . . . 159 
B.9 Lower bound on $E\left[r_{s}^{*} \mid \mathcal{S}_{1}^{c}\right]$ when $\epsilon_{p}=\epsilon_{p}^{0}$ in ATPC with ON-OFF power control at the primary user . . . . . . . . . . . 160

B.10 Conclusion for throughput scaling in ATPC case . . . . . . . . . . . . 162

B.11 Proof of Lemma 3.3.3 . . . . . . . . . . . . . . . . . . . . . 163

B.12 Expression of $\delta\left(P_{o}, c_{o}, \gamma_{\epsilon}\right) \ldots \ldots \ldots \ldots$. . . . . . . . . . . . . . . . .

B.13 Lower bound on $E\left[r_{s}^{*} \mid g \geq g_{T}\right]$ when $\epsilon_{p}=\epsilon_{p}^{0}$ for PTPC with ON-OFF power policy at the $\mathrm{PU} \ldots \ldots$. . . . . . . . . . . 165

B.14 Conclusion for throughput scaling in PTPC case . . . . . . . . . . 166

B.15 Proof of Theorem 3.4.1 for $\epsilon_{p}=\epsilon_{p}^{0} \ldots \ldots$. . . . . . . . . 167

B.16 Proof of Theorem 3.4.1 for $\epsilon_{p}>\epsilon_{p}^{0} \ldots \ldots$. . . . . . . . . 167

B.17 Proof of Theorem 3.4.2 for $\epsilon_{p}=\epsilon_{p}^{0}$. . . . . . . . . . . . . . . . . 169

B.18 Proof of Theorem 3.4.2 for $\epsilon_{p}>\epsilon_{p}^{0} \ldots \ldots$. . . . . . . . . . . 169

$\begin{array}{lr}\text { C Proofs in Chapter } 4 & 171\end{array}$

C.1 Proof of Lemma 4.2.1 . . . . . . . . . . . . . . . . . . . 171

C.2 KKT conditions corresponding to Problem (4.9) . . . . . . . . . . . 172

C.3 Proof of Lemma 4.2.3 . . . . . . . . . . . . . . . . . . . . . . . 172

C.4 KKT conditions corresponding to Problem (4.20) . . . . . . . . . . 173

C.5 Proof of Lemma 4.3.1 . . . . . . . . . . . . . . . . . . 174

C.6 Proof of convexity of the set $\mathcal{Q}_{C}\left(\mathbf{r}_{o}\right) \ldots \ldots$. . . . . . . . . . 174

C.7 KKT conditions corresponding to Problem (4.43) . . . . . . . . . 175

C.8 Proof of Proposition 4.3.2 . . . . . . . . . . . . . . . 176

C.9 Proof of Lemma 4.3.3 . . . . . . . . . . . . . . . . . . 177

C.10 Proof of convexity of the set $\mathcal{Q}_{I}\left(\mathbf{r}_{o}\right) \ldots \ldots \ldots \ldots$

C.11 KKT conditions corresponding to Problem (4.56) . . . . . . . . 178

C.12 Proof of Proposition 4.3.4 . . . . . . . . . . . . . 179

$\begin{array}{lr}\text { D Proofs in Chapter 5 } & 181\end{array}$

D.1 Proof of Lemma 5.2.1 . . . . . . . . . . . . . . . . . . . . 181

D.2 All KKT conditions for a locally optimal solution . . . . . . . . . . . 182

D.3 Proof of Lemma 5.2.2 . . . . . . . . . . . . . . . . . . . 183

D.4 GLA+SFA . . . . . . . . . . . . . . . . . . . 185

D.5 All KKT conditions for ZFLP . . . . . . . . . . . . . . . . . 185

D.6 Proof of Lemma 5.3.1 . . . . . . . . . . . . . . 186

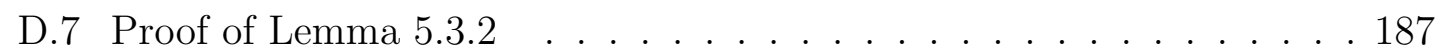

D.8 Proof of Lemma $5.3 .3 \ldots \ldots \ldots \ldots$

D.9 Expressions of $\mathcal{F}_{\text {POC }}(\theta, k)$ and $\mathcal{F}_{\text {ATPC }}(\theta, k) \ldots \ldots$. . . . . . 188 


\section{List of Figures}

1.1 Australian radiofrequency spectrum allocation chart in 2009 by ACMA. 2

1.2 Block diagram of thesis structure and contribution items. . . . . . . . 16

2.1 SU outage probability performance from SU outage probability minimization problem with POCs with varying $r_{s}^{0}\left(\epsilon_{p i}=0.1, \epsilon_{s}=0.1, r_{p i}^{0}=\right.$

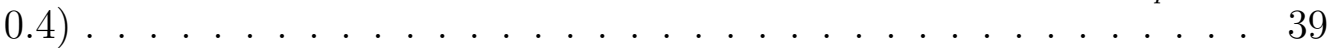

2.2 SU outage probability performance from SU outage probability minimization problem with POCs with varying $r_{p i}^{0}\left(\epsilon_{p i}=0.1, \epsilon_{s}=0.1, r_{p i}^{0}=\right.$

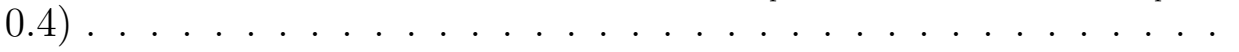

2.3 SU outage capacity versus average power budget from SU outage probability minimization problem with 4 subchannels $\left(\epsilon_{p i}=0.1, \epsilon_{s}=\right.$ $\left.0.1, r_{p i}^{0}=0.4\right) \ldots \ldots \ldots \ldots \ldots \ldots \ldots \ldots \ldots \ldots \ldots \ldots \ldots$

2.4 Average SU rate performance from SU Ergodic capacity maximization problem with $\mathrm{POC}$ and SOC with related bounds $\left(\epsilon_{p i}=0.1, \epsilon_{s}=\right.$ $\left.0.1, r_{p i}^{0}=0.4, r_{s}^{0}=0.6\right) \quad \ldots \ldots \ldots \ldots \ldots \ldots \ldots$

2.5 Average SU rate performance from SU Ergodic capacity maximization problem with POC and SOC with varying $r_{s}^{0}\left(\epsilon_{p i}=0.1, \epsilon_{s}=0.1, r_{p i}^{0}=\right.$ $0.4 \ldots \ldots \ldots \ldots \ldots \ldots$

2.6 The effect of the noisy primary channel estimates on SU ergodic capacity 44

2.7 Performance comparison between optimal and proposed suboptimal power strategies $(N=16) \ldots \ldots \ldots$. . . . . . . . . . 45

3.1 System model for cognitive BC . . . . . . . . . . . . . . . . . . 51

3.2 Region in ATPC case given that $g \geq g_{T}$ when the PU uses ON-OFF power strategy . . . . . . . . . . . . . . . . . . 59

3.3 Region in PTPC case given that $g \geq g_{T}$ when the PU uses ON-OFF power strategy . . . . . . . . . . . . . . 62

3.4 SU ergodic sum capacity in BC problem against average transmit power budget : $r_{p}^{0}=1.25, \epsilon_{p}=0.1$, and $P_{c}=15 \mathrm{~dB}$. with $\mathrm{ON}-\mathrm{OFF}$ power policy at the $\mathrm{PU} \ldots \ldots . \ldots 68$

3.5 SU ergodic sum capacity in BC problem against peak transmit power budget : $r_{p}^{0}=1.25, \epsilon_{p}=0.1$, and $P_{c}=15 \mathrm{~dB}$. with ON-OFF power policy at the $\mathrm{PU} \ldots \ldots \ldots \ldots \ldots$

3.6 Normalized SBS sum throughput with ON-OFF power policy at the $\mathrm{PU} \ldots \ldots \ldots \ldots \ldots 70 \ldots \ldots \ldots$

3.7 The effect of related parameters on SBS sum throughput with POC against $M$ for ON-OFF power policy at PU and ATPC . . . . . . . 71

3.8 The effect of related variables on SBS sum throughput with POC against $M$ for ON-OFF power policy at PU and PTPC . . . . . . . . 72

3.9 Normalized throughput in TCI power policy at the PU . . . . . . . . 72 
3.10 The effect of related variables on SU throughput scaling with POC against $M$ with TCI at PU. for ATPC . . . . . . . . . . . . 73

3.11 The effect of related variables on SU throughput scaling with POC against $M$ with TCI at PU. for PTPC . . . . . . . . . . . . . 74

4.1 System model for cognitive MAC . . . . . . . . . . . . . . . . . . 79

4.2 SU ergodic sum capacity against average power budget per user : $P_{c}=15$ dB., $r_{p}^{0}=0.3$, and $\epsilon_{p}=0.1 \ldots \ldots . \ldots . \ldots . . \ldots 109$

4.3 Ergodic-capacity region corresponding to feasible power control policy for the problem without POC and with POC: $\epsilon_{p}=0.1, P_{c}=15 \mathrm{~dB}$, $P_{a v, i}=15$ dB. . . . . . . . . . . . . . . . . 109

4.4 Common outage probability against average power constraint per user: $P_{c}=15 \mathrm{~dB} ., \epsilon_{p}=0.1, M=2, \mathbf{r}=\left[\begin{array}{ll}0.3 & 0.3\end{array}\right] \ldots \ldots . . . .110$

4.5 Common outage probability against average power constraint per user: $P_{c}=15 \mathrm{~dB} ., r_{p}^{0}=0.25, M=2, \mathbf{r}=\left[\begin{array}{ll}0.3 & 0.3\end{array}\right] \ldots \ldots 111$

4.6 Common outage probability against average power constraint per user with $M=1$ and $M=2: P_{c}=15$ dB., $r_{p}^{0}=0.25, r_{o, i}=0.3 \ldots 112$

4.7 Common outage probability and individual outage probability performance against average power constraint per user: $P_{c}=15 \mathrm{~dB}$, $r_{p}^{0}=0.25, M=2, \mathbf{r}=\left[\begin{array}{ll}0.3 & 0.3\end{array}\right] \ldots \ldots \ldots \ldots$

4.8 Individual outage probability performance against average power constraint per user: $P_{c}=15 \mathrm{~dB} ., M=2, r_{o, i}=0.3 \ldots \ldots . . . . .114$

4.9 Effects of optimal and suboptimal decoding strategies on the SU individual outage probability and actual PU's outage probability: $P_{c}=15$ dB., $M=3, r_{p}^{0}=0.25, r_{o, i}=0.4 \ldots \ldots 115$

5.1 System Model in Chapter 5 . . . . . . . . . . . . . . . . . . 120

5.2 Channel partition structure with power levels for outage minimization

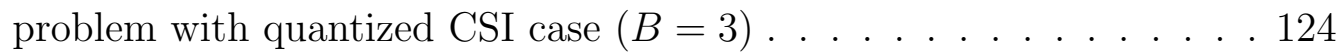

5.3 SU outage probability against average power $P_{a v}$ with varying number of feedback bits by the three algorithms . . . . . . . . . . . . . . 131

5.4 SU outage probability against average power $P_{a v}$ with varying $r_{p}^{0} \ldots 132$

5.5 SU outage probability from ZFLP and from asymptotic analysis against average power $P_{a v} \ldots \ldots \ldots \ldots \ldots \ldots$. . . . . . . . . . . . . . . . . . .

5.6 SU outage probability from ZFLP and from asymptotic analysis against

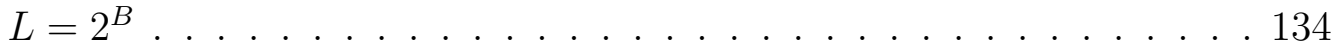




\section{List of Tables}

2.1 Three possible cases in $i$-th subchannel for a given $\boldsymbol{\nu}$. . . . . . . . . . 35

3.1 Throughput analyses in Chapter $3 \ldots \ldots$. . . . . . . . . . . 50

3.2 Four possible cases for the fading channel state $\boldsymbol{\chi}$ with ATPC and ON-OFF power policy at the $\mathrm{PU} \ldots \ldots . \ldots 56$

3.3 Four possible cases in fading channel state $\chi$ for PTPC and ON-OFF power policy at the $\mathrm{PU} \ldots \ldots . \ldots . \ldots 57$

3.4 Three possible cases in fading channel state $\chi$ for ATPC with TCI power policy at the $\mathrm{PU} \ldots \ldots$. . . . . . . . . . . 64

3.5 Three possible cases in fading channel state $\chi$ for PTPC with TCI power policy at the PU . . . . . . . . . . . . . . . . . 64

4.1 Three possible cases in common outage problem for a given $\boldsymbol{\nu}$. . . . 101 


\section{List of Acronyms}

$\begin{array}{ll}\text { AIPC } & \text { Average interference power constraint } \\ \text { AWGN } & \text { Additive white Gaussian noise } \\ \text { ATPC } & \text { Average transmit power constraint } \\ \text { BC } & \text { Broadcast channels } \\ \text { BF } & \text { Block-fading } \\ \text { C-BC } & \text { Cognitive radio broadcast channels } \\ \text { C-MAC } & \text { Cognitive radio multiple-access channels } \\ \text { CDF } & \text { Cumulative distribution function } \\ \text { CPS } & \text { Channel partition structure } \\ \text { CSI } & \text { Channel state information } \\ \text { CSIT } & \text { CSI at the transmitter } \\ \text { CR } & \text { Cognitive radio } \\ \text { D-TDMA } & \text { Dynamic time-division-multiple-access } \\ \text { ESC } & \text { Ergodic sum capacity } \\ \text { i.i.d. } & \text { independent and identically distributed } \\ \text { KKT } & \text { Karush-Kuhn-Tucker } \\ \text { NNC } & \text { Nearest-neighbourhood condition } \\ \text { MAC } & \text { Multiple-access channels } \\ \text { MIMO } & \text { Multiple-input-multiple-output } \\ \text { MISO } & \text { Multiple-input-single-output } \\ \text { OFDM } & \text { Orthogonal frequency-division multiplexing } \\ \text { OFDMA } & \text { Orthogonal frequency division multiple access } \\ \text { PCLC } & \text { Primary capacity loss constraint } \\ \text { PDF } & \text { Probability density function } \\ \text { PIPC } & \text { Peak interference power constraint } \\ & \end{array}$


POC Primary user's outage probability constraint

PR Primary receiver

PT Primary transmitter

PTPC Peak transmit power constraint

PU Primary user

QoS Quality of Service

SBS Secondary base station

SDR Software defined radio

SEC Secondary user's ergodic capacity

SNR Signal-to-noise ratio

SO Secondary user outage probability

SOC Secondary user's outage probability constraint

SPSA Simultaneous Perturbation Stochastic Approximation

SR Secondary receiver

ST Secondary transmitter

SU Secondary user

TCI Truncated channel inversion

WF Water-filling 


\section{Chapter 1}

\section{Introduction}

In this chapter, we will first briefly describe the basic concepts of cognitive radio networks and related works to pave the way for the more detailed contributions of the thesis in the subsequent chapters. In Section 1.1, we provide the rationale behind cognitive radio (CR) technology. Section 1.2 is divided into two parts. Section 1.2.1 will give an overview of information theoretic notions utilized to constitute important performance measures in general wireless communication networks and also address some important works related to this thesis. In Section 1.2.2, related literature in the context of energy-efficient resource allocation problem for different CR network paradigms will be discussed. Section 1.3 completes this chapter with an organization of the thesis and its contributions.

\subsection{Motivation}

The significance of ubiquitous wireless access has been dramatically increasing in the last decade since new wireless applications become more and more popular. For example, a mobile phone is not just a voice-only phone as we can enjoy watching video, talking to friends from different parts of the world or even conducting financial transactions through this device. Undoubtedly, the availability of high quality wireless services has fueled the acute growth in mobile subscriber demands. However, the transition from voice-only communications to multimedia communications along with the increase in the number of wireless subscribers has made wireless spectrum severely crowded. According to the Australian radiofrequency spectrum allocation chart in Fig.1.1, almost all frequency bands have been licensed and very

little new bandwidth remains for the rising number of wireless products [1]. The 


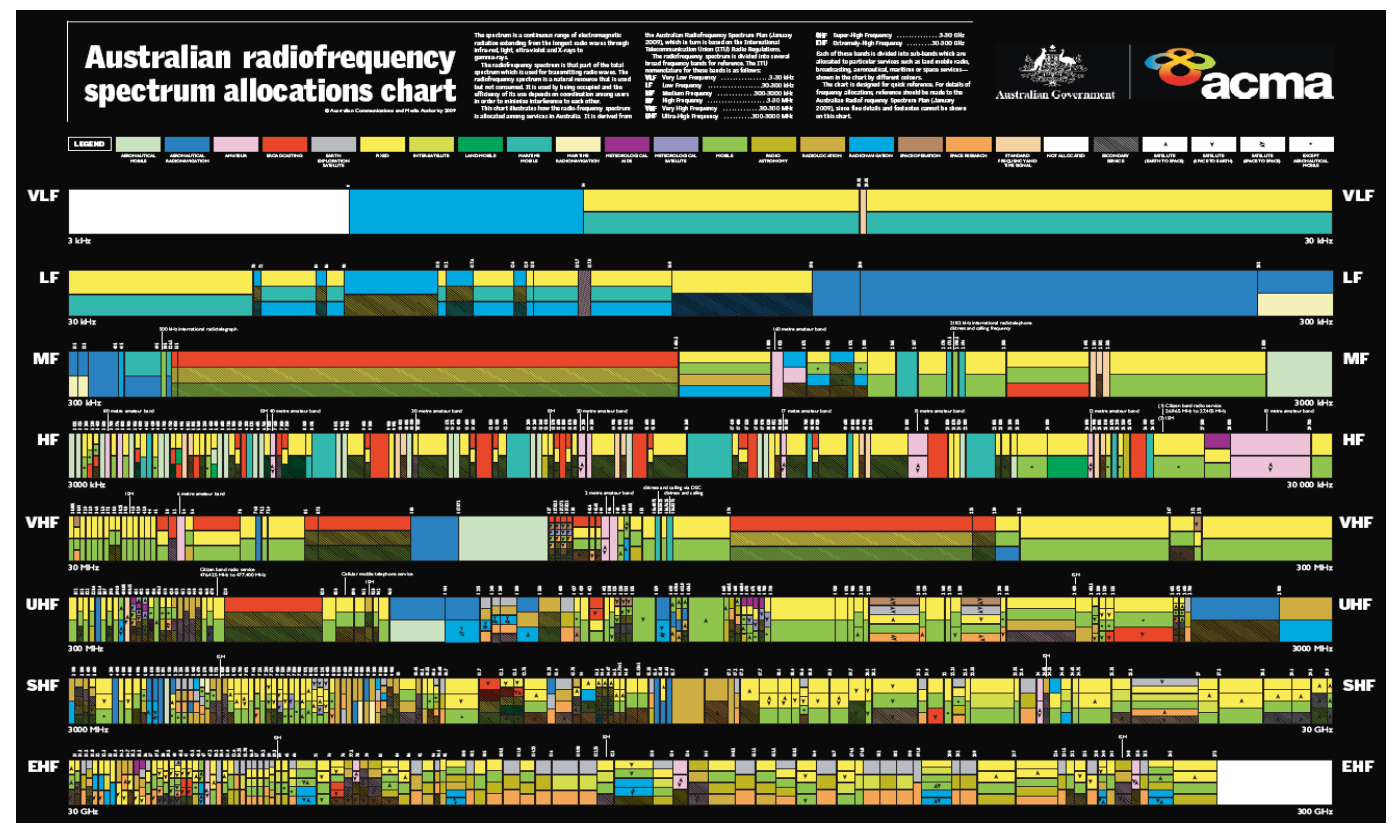

Figure 1.1: Australian radiofrequency spectrum allocation chart in 2009 by ACMA.

main benefit of the licensing approach is that the licensee can completely control its assigned spectrum and unilaterally manage interference among the users and the users' quality-of-service (QoS). Nevertheless, the bursty nature of most data traffic still provides a great deal of opportunities to use the vacant spectrum, even though a licensed channel is actively used. Furthermore, Spectrum Policy Task Force has recognized that most of licensed spectrum bands are actually not in short supply and in fact they are quiet most of the time. This implies that the spectrum drought, as perceived today, is mainly because of the static licensed spectrum management policy rather than any physical shortage [2]. In 2003, the Federal Communication Committee suggested a new concept for dynamically allocating the spectrum to encounter the problem of spectrum scarcity in the future. The idea is that additional wireless devices can exploit sophisticated technology to opportunistically share the same spectrum with the entrenched licensed users or primary users (PUs) and thus facilitate a more efficient spectrum utilization.

The cognitive radio concept can trace its roots back to an early work by Joseph Mitola in 1999. In [3], Mitola defined the term software defined radio (SDR). SDRs 
are wireless devices that can be rapidly upgraded and adapted to the environment in real time, empowered by a flexible software architecture. In [4], Mitola then coined the term cognitive radio for the SDRs that are capable of sensing the environment, for example channel, codebook, or activity side information of the other nodes with which they share the spectrum [5-7]. By exploiting the side information, the cognitive radio users can either utilize the spectrum when they sense the spectrum hole or simultaneously operate as long as their interferences do not degrade the QoS of the primary transmission to an unacceptable level. Clearly, the cognitive radio users' performances are not only bounded by their own resources, but also restricted by the service quality in the primary networks. So, it is necessary for cognitive radio users or secondary users (SUs) to wisely manage their resources in order to achieve maximum performance without disturbing service quality in primary links. To this end, several optimization techniques are applied as main tools to tackle resource management problems, making optimization one of the most attractive methodologies in this research arena.

This thesis focuses on the resource allocation problem in cognitive wireless networks where the transmission power budget is one of the limited resources. Essentially in cognitive radio networks, the service quality in the primary network also restricts the interference caused by the secondary network. Thus, the design of efficient power allocation strategies for the secondary network also depends upon the specific type of wireless service in the primary network. In many common wireless networks, real-time applications such as voice and video services constitute an important part of the overall traffic. This motivates us to investigate optimal power allocation schemes for maximizing information theoretic performance in various types of secondary networks under the QoS requirements in delay-sensitive primary networks.

\subsection{Literature review}

This section is divided into two parts. The first part will provide a review of the existing literature related to information theoretic performance measures used in 
fading wireless channels. The second part will discuss some selected articles that previously dealt with power allocation policies in cognitive radio networks, especially for the underlay paradigm based cognitive radio network, which is the cognitive radio paradigm of interest in the thesis.

\subsubsection{Capacity in wireless channel}

The channel capacity is one of the most important performance measures in wireless communication as it represents the maximum data rate can be transmitted through the wireless channel with asymptotically small error probability. The breakthrough study in channel capacity was pioneered in 1948 by Shannon based on the notion of the mutual information between input and output of a channel [8]. More specifically, capacity is defined as the mutual information maximized over all possible input distributions. However in practical situations, a wireless channel varies randomly and continuously over time and the transmitters cannot have infinite power budget. Wolfowitz then considered the capacity of time-varying channel, proving that if the channel stochastic process is assumed to be stationary and ergodic, the capacity of the time-varying channel is the expectation of the capacity of the particular channel state [9] and the result is the same even though the number of channel state is infinite [10]. Under the assumption of stationarity and ergodicity, the maximum achievable capacity assuming no constraints on delay is thus called the ergodic capacity. In a single user scenario, [10] argued that if the transmitter and the receiver know the channel state, i.e. perfect channel side information (CSI) is assumed, the transmitter can adapt its power to achieve the maximum ergodic capacity subject to its average power constraint. The optimal power control scheme is the well-known water-filling (WF) policy. The idea is to allocate more power to the better channel power gain while stop transmitting when the channel power gain is really poor [11]. After [10], there were several works that looked at ergodic capacity maximization problems in various sorts of wireless networks. In orthogonal frequency-division multiplexing (OFDM) technology where the transmitter sends information through parallel subchannels, the optimal power policy to achieve maximum ergodic capacity subject to its average power constraint is also in water-filling manner, i.e. water-filling is done 
over the OFDM subcarriers, alias multi-dimension water-filling [12]. In single-input single-output (SISO) fading multiple-access channels (MAC) with continuous fading channel scenario, [13] showed in 1995 that the base station allocates a given time slot (over which the fading channel remains invariant) to the user with the strongest channel power gain only so as to maximize the ergodic uplink sum capacity in a MAC with identical transmitters, implying that a dynamic time-division-multiple-access (D-TDMA) is the optimal scheme. In 1998, [14] studied the analogous scenario but investigated more general cases by considering unequal rate rewards in each user for the sake of fairness among users. By exploiting the convexity and the polymatroid structure of the problem, [14] rigorously proved that each point on the boundary of the ergodic capacity region is attainable by successive decoding. Coincidentally, Tse proposed the optimal power allocation scheme for maximizing ergodic sum downlink capacity in SISO fading broadcast channels (BC) in 1997, showing that D-TDMA is optimal by designating the whole time slot for the user with the strongest reception only [15]. Then in 2001, [16] investigated the ergodic capacity region of $M$-user fading $\mathrm{BC}$ with perfect CSI at the base station and the receivers. These results are obtained for code-division with and without successive decoding, frequency-division and time-division, showing that the achievable regions for frequency-division, timedivision and code-division without successive decoding are equivalent whereas the achievable region for code-division with successive decoding has the largest capacity region.

However, ergodic capacity is the performance measure of the long-term achievable rate averaged over the time-varying channel. To average out the fluctuation of the channel, the coded symbols must span many coherence time periods, and this coding/decoding delay can be quite significant [12]. For applications that have a tight delay constraint relative to the channel coherence time, this notion of capacity is not meaningful because the user may suffer from outage. The outage event happens when the instantaneous rate drops below the encoding rate or target rate at the receiver. Then, the decoding error probability at the receiver cannot be made arbitrarily small regardless of the code used by the transmitter [12]. The probability of the outage event for the corresponding target rate is called the outage probability. 
In the delay-limited case, we restrict ourselves to power control policies such that the instantaneous mutual information is kept constant at all times, i.e. zero outage probability. The notion of delay-limitedness is inferred in many articles. Several works on power control, such as [17] and [18], assume that a desired signal-to-interference noise ratio must be met for every fading realization so as to the encoding rate is kept constant. While it is straightforward that the single user delay-limited power control policy is channel inversion, the multiple user scenario is more interesting since it involves tradeoffs between the power and rate allocation and the interference among the users. In [19] the authors defined the formal concept of delay-limited capacity, also known as zero-outage capacity, for MAC. Similar to [14], [19] utilizes the convexity and polymatroid structure of the problem to characterize the entire delaylimited capacity region and the corresponding optimal power control schemes and proves that the optimal solution is always successive decoding. Nonetheless, there are some fading distributions for which zero outage probability cannot be achieved, such as Rayleigh fading, since the probability that the channel is in deep fade is non-zero [12]. Another performance measure called the $\epsilon$-outage capacity is also used to define the largest rate of transmission $R$ such that the outage probability is less than $\epsilon$. In the single-user case, the well-known power strategy, called truncated channel inversion (TCI), is the optimal solution to $\epsilon$-outage capacity maximization under average transmit power constraint [20]. In [21], the authors solved the outage minimizing problem for the $M$-parallel block-fading additive white Gaussian noise (BF-AWGN) channel with perfect CSI assumed at both transmitter and receiver. The optimal power solution suggests that deterministic schemes are generally suboptimal but a probabilistic power allocation scheme is optimal for channel distribution consisting of discrete fading states, i.e. the optimal solution is randomized between zero-power and a multi-dimensional basic rate allocation policies. In [22] the authors studied two outage scenarios for the SISO fading MAC. The first one is common outage where an outage must be declared simultaneously for all users and the second one is individual outage where an outage can be declared individually. Significantly, it was shown in [22] that finding the outage capacity region is equivalent to deriving the outage probability region for a given rate vector. Similar to the results in [14] 
and [19], [22] also proved that successive decoding is the optimal decoding strategy. In SISO fading BC, [23] looked at several types of time-division multiple-access similar to [16] and proved by the same technique as in [22] that the outage capacity region can be implicitly acquired by deriving the outage probability region for a given rate vector. The results presented in [13] and [15], in [14] and [16] and in [22] and [23] reflect the hidden relationship between MAC and BC problems. The relationship is later clarified in [24] which emphasized the duality of the capacity region of Gaussian MAC and BC by showing that the capacity region of the Gaussian $\mathrm{BC}$ under a sum power constraint is exactly the same as the capacity region of a dual Gaussian MAC subject to the same sum power constraint instead of individual power constraints.

In [25], the authors argued that neither the ergodic capacity nor the outage capacity is exclusively appropriate in some variable rate applications. For example in some video or audio applications, the source rate can be adjusted due to the fading channel conditions in order to provide multiple QoS levels. Generally, a non-zero basic rate $R$ is needed to ensure a minimum acceptable service quality. Therefore, the authors solved the problem of the ergodic capacity maximization subject to an average power constraint and the constraint that the outage probability is no more than $\epsilon$ for a given target instantaneous rate $R$. Note that the outage probability constraint is imposed on the problem in order to guarantee the minimum QoS. The problem is also known as service-outage maximization problem, for an $M$-parallel block fading channel. The derivation reveals that the optimal power strategy is randomized between two deterministic power allocation schemes [25], i.e. a multi-dimensional water-filling allocation and a multi-dimensional basic-rate allocation scheme. In free-space optical communication channel, [26] examined the service-outage maximization problem by suggesting that intensity modulation direct detection optical block fading channel with Poisson receiver statistics can be modelled as Poisson fading channel and again the optimal power solution shows the randomization manner between two deterministic schemes. Since outage probability is used as the QoS metric for delay-sensitive users in this thesis, the idea of probabilistic power allocation technique shown in $[21,25,26]$ serves as a vital tool to help 
solving problems from Chapter 2 to Chapter 4.

\subsubsection{Cognitive radio network paradigms}

In cognitive radio networks, the QoS of primary networks must be guaranteed, making power control schemes in non-cognitive environments unable to be directly applied. Based on the type of available network side information, there are three main approaches for $\mathrm{CR}$ to manage its resultant interference: interweave, overlay, and underlay $[6]$.

The interweave or spectrum sensing paradigm is regarded as the simplest approach to prevent secondary networks from degrading the QoS in primary network by allowing SUs to transmit only the frequency band is detected to be idle. The idea is originally outlined in [4]. In this paradigm, occupancy knowledge of the primary users in each spectrum is required for the cognitive transmitters, making an accurate sensing of the presence of non-cognitive users quite crucial. Theoretically, the resultant interference from secondary networks is zero when primary network is active. In practice, sensing ability is imperfect due to multipath fading of wireless channels and the fluctuations in noise/interference level [27], leading to the the occurrence of probability of missed detection and probability of false alarm. Also, the frame structure of any cognitive radio system consists of a sensing time slot and a data transmission slot, leading to the tradeoff between sensing time and data transmission time [28]. Motivated by these limitations, the topic on resource allocation in the interweave paradigm becomes attractive. For example, [29] considers the achievable throughput from a queue stability perspective by deriving the optimal power control for maximizing the cognitive users arrival rate whereas PU's packet arrival is held fixed to assure a certain primary average throughput. The optimal sensing time and power allocation that maximizes the average throughput subject to the probability of detection of the PUs under two different power constraints: peak (short-term) transmit power constraint and average (long-term) transmit power constraint were derived in [28] for a single frequency band and in [30] for a wideband scenario.

For the overlay paradigm, the codebook and message side information of primary networks are presumed to be available at secondary networks. Thus, concurrent 
cognitive and non-cognitive users transmissions are allowed and the cognitive radio users may even facilitate the transmission of the non-cognitive users. The overlay paradigm is also referred in the information theory literature as an interference channel with asymmetric message knowledge, degraded message sets, or one cooperating encoder [6]. One of the notable works on the overlay paradigm is [7] which addressed four causal protocols and investigated corresponding achievable rate regions of cognitive and non-cognitive users which combines Gel'fand-Pinkser coding [31] with an achievable rate region construction for the interference channel [32]. In the additive Gaussian noise case, this represents the similarity to dirty-paper coding [33], a technique used in the computation of the capacity of the Gaussian multiple-input multiple-output (MIMO) BC [34]. Providing that there is no rate degradation for the PU in the SU's vicinity and the primary receiver adopts a single-user decoder, [35] characterized the largest reliable rate for the SU and also demonstrated that multiuser decoding at the primary receiver is optimal in the high-interference regime for the viewpoint of maximal jointly achievable rates in the overlay network with a pair of primary and secondary users.

In the underlay paradigm $[6,36]$ or horizontal spectrum sharing $[5,7]$, which is the main paradigm of interest in this thesis, cognitive networks can simultaneously share the same spectrum with primary networks. Unlike the overlay paradigm, the cognitive radio users in underlay paradigm are assumed to have CSI of the network. By exploiting channel knowledge, the cognitive radio users must control the amount of their interference to avoid deteriorating the QoS of the primary networks to deficient level. The simplest method is to limit interference from secondary network to each primary receiver or the so-called interference temperature [5]. The idea of interference temperature limit is to quantify and manage the source of interference by real-time interactions between transmitter and receiver in an adaptive manner so as to ensure that the total interference from the secondary network to each primary receiver is no more than a predefined value. So, given a particular frequency band in which the interference temperature is not exceeded, that band could be made available to cognitive radio users [5]. Motivated by the idea of interference temperature limit, the study on the channel capacity for secondary users in underlay paradigm 
has drawn a lot of attention. Gastpar derived the behaviour of capacity of different additive white Gaussian noise (AWGN) channels subject to a received power constraint at the intended receiver or at the third party in [37]. The capacity under receive power constraints at the receiver side is shown to be very similar to that under transmit power constraints at the transmitter side because, for such invariant channels, the received power at the third-party receiver is merely a deterministically scaled version of the transmitted power. Therefore, analyzing the channel capacity problem subject to received power constraints is tantamount to analyzing it subject to transmit power constraints. However in fading wireless channels, the secondary network can gain benefits from the presence of channel fading, for example when the channel gain from a secondary transmitter to a primary receiver is in deep fade, it is more likely that the secondary transmitter can opportunistically increase transmit power to achieve higher rate.

Based on the results of [37] and the fading property of typical wireless channels, a number of works have addressed the design of optimal power allocation policy to achieve maximum transmission performance in the secondary network. For point-topoint communication in the underlay paradigm, [38] studied the SU ergodic capacity maximization subject to either an average or a peak interference power constraint at each single-antenna equipped primary receiver. The corresponding power control policies were derived and later used to compute the closed-form expression of the ergodic capacity in various types of channel fading statistics. Nevertheless, [38] overlooked the effect of transmit power budget which is a limited resource for any wireless devices in practice. Under the identical problem considered in [38], [39] also imposed transmit power constraint at the secondary transmitter, i.e. either an average transmit power constraint or a peak transmit power constraint, and analyzed both ergodic capacity maximization and outage probability minimization problems under different channel fading scenarios. In 2009, [40] investigated the analogous scenario but assumed that the SU utilizes sensing-based spectrum sharing approach to avoid exceeding the average interference threshold at the primary receiver. More specifically, the model consists of two stage for each frame duration time $T$. In the first stage, the SU listens to activity of the PU with sensing time $\tau$. In the second 
stage, the SU adapts its transmit power based on the sensing results in the remaining duration time $T-\tau$. The optimal power allocation policies for both when PU is active and when PU is inactive and optimal sensing time $\tau$ were derived. In [41], authors derived the optimal power allocation scheme that maximizes secondary user's effective capacity [42] subject to an average interference constraint (the simplified version of outage probability constraint at the PU) in Rayleigh fading channel which ensures the QoS of the delay-sensitive PU.

Several articles, such as $[43,44]$, have also suggested that the secondary networks can further increase transmission performance by exploiting CSI between secondary transmitter and primary receiver channels and between primary transmitter and primary receiver channels. For secondary transmitter-primary receiver channels, it is suggested that the secondary transmitter can estimate this gain by measuring the received power of signals transmitted by the primary receiver and under the assumption of channel reciprocity and that secondary transmitter knows the primary receiver transmission power. For primary transmitter-primary receiver channels, various suggestions have been made including that of eavesdropping on primary receiver feedback to primary transmitter [45], and receiving feedback from a cooperative SU node employed near the primary receiver [46]. Instead of using an average interference power constraint to guarantee PU's QoS, a new constraint called primary capacity loss constraint (PCLC) was introduced into SU ergodic capacity maximization problem [43]. The idea is that the SU will not cause an unsatisfactory loss in PU's ergodic capacity if it knows an additional CSI between the primary transmitter and the primary receiver. The solution for the problem is called modified water-filling power control since the power level is adapted based on not merely the channel power gain between the secondary transmitter-receiver pair, but also the channel power gains between primary receiver and secondary transmitter and between the primary transmitter-receiver pair. By using Jensen's inequality, the author also showed that the proposed SU power control policy is superior to the conventional strategy under interference constraint by showing that the achievable ergodic capacity region of PU and SU under PCLC is larger than that under an average interference power constraint. Although considering PCLC instead of aver- 
age interference power constraint gives an advantage to secondary network, PCLC is not an appropriate constraint when PU is interested in a delay-sensitive application, such as live video streaming, because ergodic capacity does not take a tight delay constraint into an account. Hence, primary user's outage probability constraint (POC) was introduced in [44] where PU's outage probability must not exceed the given threshold. The optimal power policies to maximize SU ergodic capacity and to minimize SU outage probability subject to POC and transmit power constraint (either peak or average power constraint) were provided in [44].

Optimal power allocation problems in the underlay paradigm are also extensively studied in other types of secondary networks. There are several articles considering a parallel fading channel in secondary network where a SU is equipped with OFDM technology. OFDM has gained reputation with the emergence of wireless communications and wideband systems because of simplicity in channel equalization and coding and the ability to compensate for multipath fading [47]. Especially in OFDM-based CR system, SUs will have more alternatives to enhance its transmission efficiency, for instance, by using the spectrum slots left by PUs [48], transmitting opportunistically through unoccupied subcarriers in the primary systems [49], or even sharing the subcarriers with PUs on the condition that the QoS of the primary system is guaranteed $[50,51]$. In [51], the authors studied channel capacity maximization of an OFDM-equipped secondary user sharing the subcarriers of the orthogonal frequency division multiple access (OFDMA) based primary system. Each subcarrier of the primary system is protected by either an interference constraint or PCLC, i.e. hybrid protection constraint. While in [49], the effect of SU cross-band interference to the side-by-side PU frequency bands was elaborated by solving the total transmission rate maximization problem subject to total cross-band interference and transmit power constraint in each subcarrier.

There are also a lot of works paying attention to the multiple-user scenario for both cognitive MAC (C-MAC) and cognitive BC (C-BC). For example, [52] extended the results from fading non-cognitive MAC in $[13,14]$ to fading C-MAC and from fading non-cognitive BC in $[15,16]$ to fading C-BC. The authors in [52] derived the optimal power policies that maximize ergodic sum capacity for both uplink and 
downlink channel for secondary network subject to average/peak transmit power constraint at each transmitter and average/peak interference power constraint at each receiver. In $\mathrm{C}-\mathrm{BC}$, it was shown that the base station allocates a given time slot to only one user so as to maximize the ergodic sum downlink capacity, implying that a dynamic time-division-multiple-access (D-TDMA) is the optimal scheme. However, it is not always the case in C-MAC. Particularly, more than one user can transmit at the same time when peak interference constraints are included in the ergodic sum uplink capacity maximization problem. Recently, [53] characterized common outage capacity region and individual outage capacity region in cognitive MAC subject to peak interference power constraint at each primary receiver and peak transmit power constraint at each secondary transmitter and solved the corresponding optimal power control schemes. By using a similar idea proposed in [22] for the non-cognitive MAC problem, [53] argued that the outage capacity region can be implicitly obtained by deriving the outage probability region for a given rate vector. Another challenging problem for the multiple-user scenario in cognitive system is to analyze how the sum throughput in the secondary network scales as the number of secondary users, $M$, grows large. Originally, Sharif and Hassibi [54] studied how the ergodic sum downlink throughput in non-cognitive multiple-inputmultiple-output (MIMO) broadcast channel scales with number of transmit antennas, number of receive antennas and number of receivers. In [54], the base station is presumed to allocate the whole time slot to the receiver with the maximum signalto-interference-noise ratio with the constant power allocation policy. Note that [54] became the first work that adopts extreme value theory to rigorously render throughput scaling analysis. Extreme value theory is utilized to specify the possible forms for the limiting distribution of maxima in sequences of independent and identically distributed (i.i.d.) random variables. Apart from the telecommunication arena, extreme value theory is also used in other aspects, such as geological engineering [55] and financial risk analysis [56]. With the aid of extreme value theory, the secondary throughput scaling is later analyzed for three types of cognitive networks, including cognitive MAC, cognitive BC, and cognitive parallel access channel, under peak transmit power and peak interference power constraints in [57]. Then, [58] examined 
the multiuser diversity gain due to the optimal power allocation policy in cognitive MAC under an average interference power constraint and an average transmit power constraint with various types of fading channel statistics.

Although the knowledge of CSI is assumed at secondary transmitters in the underlay paradigm, to obtain perfect CSI theoretically requires infinite feedback bits from receivers even in non-cognitive networks, making the assumption impractical. Therefore, various works looked at power allocation problems using limited feedback scenario. Many results, for example in [59-61], even attested that allowing the receiver to feedback a small number of bits to the transmitter can make the performance of the system approach the optimal performance with full CSI. In [59], outage minimization problem subject to average transmit power constraint for a single-user non-cognitive MIMO system with quantized feedback was investigated. With a given set of power levels, the optimal channel state partitioning can be derived by using the nearest-neighbourhood condition (NNC) and the problem can be rewritten as a function of power. Then, the optimal power control can be derived by using Karush-Kuhn-Tucker (KKT) necessary condition. By using NNC and KKT conditions, several papers used this approach to solve the optimization problem with quantized feedback in various types of networks. In [62], outage probability minimization subject to average transmit power constraint in parallel fading channels was solved. In cognitive radio scenarios, SU ergodic capacity maximization problem in parallel channel subject to average transmit power constraint at the SU and average interference power constraint at PU in each subchannel was addressed in [60] and the optimal power policy was derived accordingly. Later, [63] investigated the same network setup as [60] but an average interference power constraint is replaced by a peak power constraint at PU in each subchannel. The SU outage probability minimizing problem in a narrow band network subject to average transmit power and average interference power constraint was derived in [61]. In [60-63], various suboptimal algorithms were elaborated to ease complexity in computational problem in the optimal solutions and asymptotic analyses of the SU performance were also studied. In multi-antenna radio networks systems, the resource allocation problem is still challenging as beamforming and power control codebooks are required to be 
optimally designed. However, existing works design these codebooks independently, i.e. either assume fixed transmission power and find the optimal beamforming codebook [64-66] or presume an isotropic transmission at the transmitter and solve the optimal power control [67-69]. Although [70] recently proposed a joint design of power control and beamforming codebook for outage minimizing problem in noncognitive single user multiple-input-single-output (MISO) network, the problem is even more complicated in cognitive radio environment due to constraint imposed by the primary networks. This makes the design and analysis of limited feedback based algorithms for coexisting multiple antenna networks a largely unchartered research arena. In [71], the authors considered the outage minimization problem of MISO system in secondary network under interference constraint and proposed two cognitive beamforming algorithms for cognitive beamforming based on quantized cooperative feedback from single-antenna equipped primary receiver to the secondary transmitter based on the orthogonality of the channel between the SU transmit beamformer and the feedback channel. Also, the effect of the cooperative feedforward of the secondary-link CSI from secondary transmitter to the primary receiver was examined.

\subsection{Outline and contribution of the thesis}

In this thesis, we focus on optimal transmission power allocation problems in specific types of secondary networks which share the same frequency band licensed to delaysensitive primary links. Throughout the thesis, an outage probability constraint is imposed at each delay-sensitive PU, i.e. the service quality in each primary link is guaranteed by a POC.

The block diagram in Fig. 1.2 illustrates the organization and the contribution items of the thesis. More specifically, the contributions to the thesis from Chapter 2 to Chapter 5 are as follows:

\section{- Chapter 2}

We consider a CR network with an OFDM-based SU and $N$ delay-sensitive PUs. The SU aims at transmitting delay-insensitive as well as delay-sensitive 


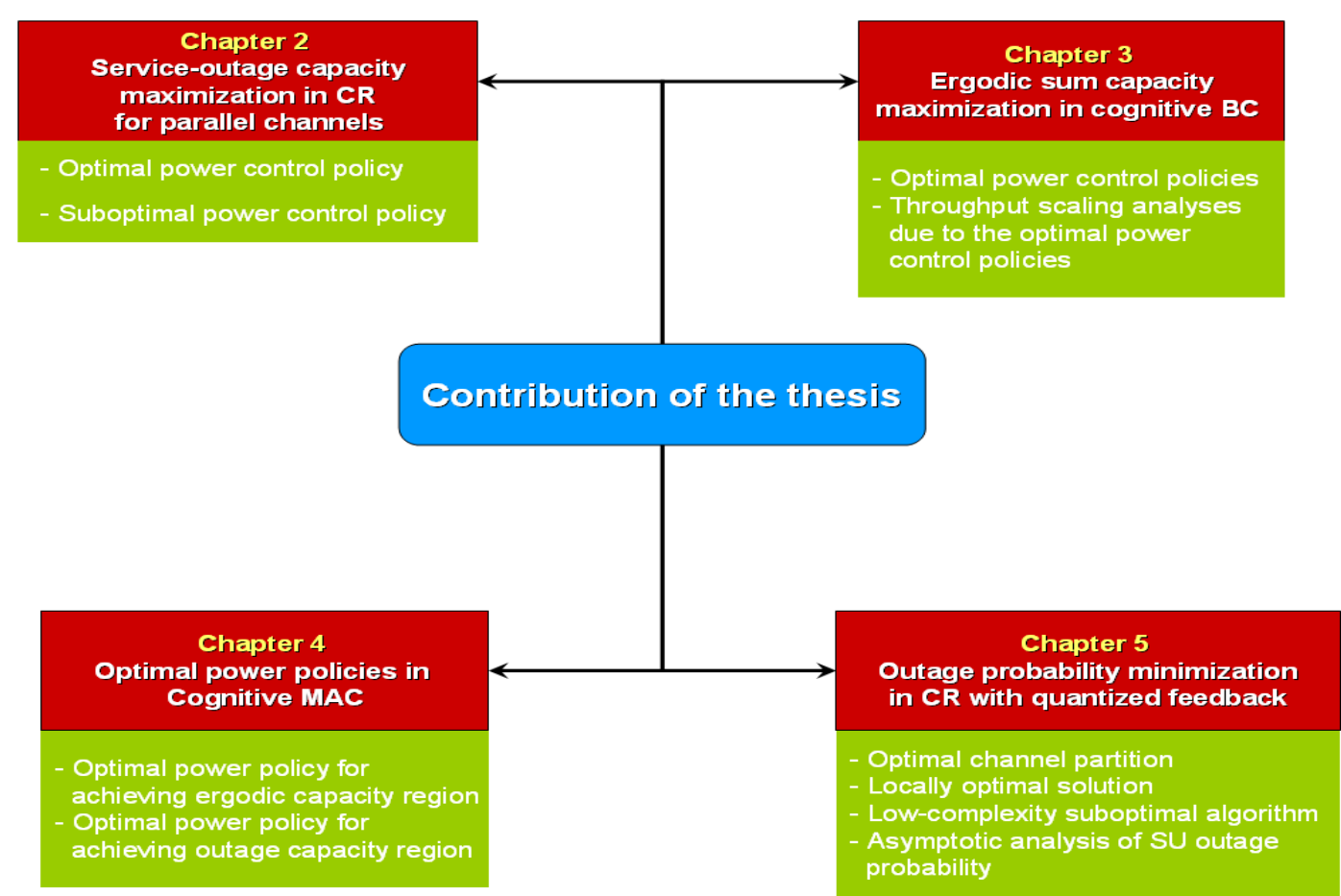

Figure 1.2: Block diagram of thesis structure and contribution items.

data by accessing $N$ randomly fading frequency bands which each band is licensed to an individual delay-sensitive PU which is interested in meeting a minimum rate guarantee for delay-sensitive services with a maximum allowable primary outage probability, i.e. POC. Typically, PUs has its own power policy based on the CSI of its direct gain between the PU transmitter and the PU receiver only regardless the existence of secondary networks. Under the assumption that PUs' power policies and CSI of the network are revealed to the SU, we solve the SU's ergodic capacity maximization problem subject to SU's average transmit power and outage probability constraints (SOC) and all POCs or the so-called service-outage based capacity maximization for SU with POCs. With a rigorous probabilistic power allocation technique, it allows us to derive optimal power policies applicable to both continuous and discrete fading channels. Also, a suboptimal power control policy is proposed in order to alleviate the high computational complexity of the optimal policy when $N$ is large. 


\section{- Chapter 3}

This chapter focuses on a spectrum-sharing based fading cognitive radio broadcast channel (BC) with a single-antenna secondary base station (SBS) and $M$ single-antenna secondary receivers (SRs) concurrently utilizing the same spectrum band with a delay-sensitive primary user (PU). The quality-of-service requirement for the primary user is set by an outage probability constraint (POC). We address the optimal power allocation problem for the SBS ergodic sum capacity (ESC) maximization in the secondary BC network subject to POC and a transmit power constraint at SBS described by either an average or a peak power constraint. Optimality conditions reveal that in each timeslot (or fading block over which the channels remain invariant) SBS will choose only one SR that has the highest value of a certain metric consisting of the ratio of the SR's direct channel gain and the sum of interference and noise at the

SR, and allocate the timeslot to that user. Furthermore, the secondary network throughput scaling analysis as the number of secondary users $M \rightarrow \infty$, is also investigated, showing that if PU utilizes an ON-OFF transmission power control policy with a constant power when ON, the SBS ESC in the cognitive $\mathrm{BC}$ scales according to $\log (\log M)$. If PU applies a truncated channel inversion (TCI) power policy (which is equivalent to the optimal power control policy for primary outage minimization in the absence of the secondary network), the SBS ESC scales like $\epsilon_{p} \log (\log M)$ where $\epsilon_{p}$ is the PU outage probability threshold.

\section{- Chapter 4}

This chapter concerns a spectrum-sharing based fading cognitive multipleaccess channel (C-MAC) with a single-antenna secondary base station (SBS) and $M$ single-antenna secondary transmitters, simultaneously sharing the same spectrum band with one delay-sensitive primary user $(\mathrm{PU})$ and aiming to communicate with the SBS. The quality-of-service in primary network is guaranteed by a primary user's outage probability constraint (POC) while an individual power resource for each secondary transmitter is limited by an average 
transmit power constraint (ATPC). This chapter considers the problem of optimal power allocation depending on whether the traffic in the secondary network is delay-sensitive or not. In delay-insensitive traffic, we first study the optimal power allocation policy that maximizes ergodic sum uplink capacity subject to POC and ATPC. Then, we extend to the more generalized case, i.e. the optimal power strategy for achieving ergodic capacity region subject to POC and ATPC. In delay-sensitive traffic, we discuss two types of outage situation, i.e. an outage must be declared simultaneously for all users (common outage) and outages are declared individually for each user (individual outage). Then, the power policies to achieve the common outage capacity region and the individual outage capacity region subject to POC and ATPC are presented. Under the assumption that each SU knows the channel state perfectly in a continuous fading channel scenario, the optimal power strategies for these problems are derived by using a rigorous probabilistic power allocation technique and the solution reveals that successive decoding is optimal and the decoding order can be solved explicitly as a function of channel state.

\section{- Chapter 5}

Finally, this chapter looks at the more practical scenario with quantized CSI presumed in the secondary network. In this chapter, a single-antenna SU coexists with a delay-sensitive and single-antenna PU in a narrowband underlay CR network and receives quantized CSI through a finite $B$-bit feedback from a band manager or a cognitive radio network manager. We consider the power allocation problem for minimizing outage probability of a single-antenna SU under POC and average transmit power constraint at SU. Exploiting a nearest-neighbourhood condition, we can derive the optimal channel partition structure (CPS) for the vector channel space, making KKT condition applicable as a necessary condition for finding a locally optimal power codebook. A low-complexity suboptimal algorithm is also proposed. Furthermore, an asymptotic analysis of the SU outage probability is also investigated.

Last but not least, Chapter 6 provides some concluding remarks as well as pos- 
sible extensions for future works. 


\section{Chapter 2}

\section{Service-outage Capacity Maximization in Cognitive Radio for Parallel Fading Channels}

Orthogonal frequency division multiplexing (OFDM) is regarded as a potential transmission technique for broadband wireless systems due to its high transmission efficiency, its robustness against inter-symbol interference in frequency selective channels, and especially due to its great flexibility in dynamically allocating transmission resources, making OFDM widely accepted as a promising candidate for future CR systems [48]. In OFDM-based CR system, SUs will have more alternatives to enhance its transmission efficiency, for instance, by using the spectrum gaps left by PUs [48], transmitting opportunistically through vacant subcarriers in the primary systems [49], or even sharing the subcarriers with PUs on the condition that the QoS of the primary system is guaranteed [50] [51].

This chapter will focus on a transmit power allocation problem in an OFDMbased CR system within the underlay paradigm where an OFDM-based SU seeks a fundamental tradeoff between maximizing its own throughput with limited resources and minimizing the performance loss in the primary system. Resource allocation problems in OFDM-based CR systems have already attracted wide attention. In [12] and [72], it is shown that the water-filling power policy is the optimal transmission strategy to maximize ergodic channel capacity in a conventional OFDM system with a total ATPC across the sub-bands . This water-filling policy cannot be used when PUs' service quality constraints are taken into an account. In [50], the authors derived the solution of SU's instantaneous rate maximization problem with transmit power constraint and an individual interference power constraint on each subcarrier to protect the corresponding primary transmission. Then in [51], the authors proposed a new type of constraint to protect the PU QoS, called the 'rate 
loss constraint' (RLC), defined as the upper bound of the PU rate loss due to SU transmission. In [49], the authors derived the optimal power control policy for the SUs' sum rate-maximizing power allocation problem under the constraint that SU cross band interference incurred by the side-by-side PU frequency bands is limited. However, if the PUs are engaged in transmission of delay-sensitive information, the PU outage probability constraint becomes a more suitable measure for protecting a delay-sensitive primary user in $[73,74]$ where the optimal power allocation problems for the secondary ergodic and outage capacity maximization problem with a primary outage constraint were addressed and later further extended in [44]. Finally, an effective capacity based delay QoS constraint on the SU and a PU outage constraint was considered in [75], where the authors propose a variable-rate variable-power based MQAM scheme to solved the associated optimization problems with full CSI.

In this chapter, we consider a CR network where an OFDM-based SU operates in an orthogonal frequency division multiple access (OFDMA) based primary system. The SU aims to transmit both delay-sensitive and delay-insensitive information (such as integrated voice/video and packet data) over $N$ subcarriers of the OFDMA-based primary system. Each subcarrier is licensed to an individual PU that wishes to maintain a basic rate with a certain outage probability. In other words, we solve the SU's ergodic capacity (SEC) maximization problem under an SU's outage probability constraint (SOC), $N$ PUs' outage probability constraints (POCs), and an SU's average transmit power constraint (ATPC). This problem is closely related to the 'service-outage capacity problem in parallel fading channel' [25] in the sense that if all $N$ POCs are discarded from our problem, the two problem become exactly the same. The idea is that as soon as the service quality of the delay-sensitive information (voice) is ensured by a guaranteed outage probability, any excess rate can be used to delay-insensitive information (data) in a best-effort fashion. The problem also extends the result in [76], where only one single frequency band is considered. Furthermore, this chapter addresses the relationship between the feasibility of the SEC maximization problem and the problem of SU's outage probability (SO) minimization subject to all $N$ POCs and an ATPC. This SO problem was initially addressed in [74] for $N=1$. In this chapter, the result of [74] is 
generalized to the case when $N>1$. Under the assumption that the PU in each subcarrier has a transmission policy that is based on its own channel between its corresponding primary transmitter and receiver only and that the SU has knowledge of all PUs' transmission policies as well as the full channel state information (CSI) of the entire network, both SEC and SO problems are solved by using a rigorous 'probabilistic power allocation' technique, originally proposed in [21]. This method allows us to treat the optimal power allocation problem as a convex optimization problem and renders our power allocation results applicable to both continuous as well as discrete fading channels. However, the optimal power control for the SEC problem results in a high computational complexity (exponential in $N$ ) when the number of sub-carriers, $N$ is large. Thus motivated, we also propose a suboptimal power control policy with a reduced real-time computational complexity to alleviate this problem. Numerical studies illustrate the performance of the optimal power policies and demonstrate that our proposed suboptimal policy is not only computationally efficient, but also satisfies the SOC and all POCs incurring a small SU ergodic capacity loss.

The rest of this chapter is organized as follows. Section 2.1 presents the system model. We formulate SEC maximization problems in Section 2.2. Section 2.3 demonstrates the derivation on the optimal solution for the SEC problem, discusses the feasibility of SEC problem and its relationship with the extended SO minimization problem, and proposes a low-complexity suboptimal power scheme for the SEC maximization problem. Illustrative numerical results are provided in Section 2.4 followed by some concluding remarks in Section 2.5.

List of notations in Chapter 2: $\frac{\partial y}{\partial x^{*}}$ denotes the derivative of $y$ over $x$ evaluated at $x=x^{*} . \prec, \preceq$ denote componentwise strict inequality and componentwise inequality in $\mathbf{R}^{N}$, respectively. $\langle\mathbf{x}\rangle=\sum_{i=1}^{N} x_{i} \cdot[x]^{+}=\max (0, x) . \mathbf{1}\{\mathscr{Z}\}$ represents indicator function, i.e. $1\{\mathscr{Z}\}=1$ if the event $\mathscr{Z}$ is true and it is zero for otherwise. 


\subsection{System model}

We consider a cognitive radio environment with $N$ primary transmitter-receiver pairs (PT-PR) and a single OFDM-based secondary transmitter-receiver pair (ST-SR). The SU can access all $N$ frequency bands of which the $i$-th band is licensed to the $i$ th $\mathrm{PU}\left(\mathrm{PU}_{i}\right)$ for $i \in\{1,2, \ldots, N\}$ in an OFDMA-based primary system. All channels involved in this cognitive radio network are assumed to be block fading additive white Gaussian noise (BF-AWGN) channels [21]. The instantaneous channel power gains in the $i$-th subchannel for the link $\mathrm{PT}_{i}-\mathrm{PR}_{i}, \mathrm{ST}-\mathrm{SR}, \mathrm{PT}_{i}$-SR, and $\mathrm{ST}_{-} \mathrm{PR}_{i}$ are denoted

by $g_{i}, h_{i}, \alpha_{i}$, and $\beta_{i}$, respectively. Let $\nu_{i} \triangleq\left[g_{i}, h_{i}, \alpha_{i}, \beta_{i}\right]$ and $\boldsymbol{\nu} \triangleq\left[\nu_{1}, \nu_{2}, \ldots, \nu_{N}\right]$ represent the combined channel state vector. The vector fading process $\boldsymbol{\nu}$ is presumed to be stationary and ergodic with a cumulative density function $F(\boldsymbol{\nu})$. The additive noises at PR and SR in $i$-th subchannel are assumed to be independent Gaussian random variables with zero mean and variance $N_{0}$. We assume that SU transmitter has full CSI of $\boldsymbol{\nu}$, i.e. all channel gains in the network, while the $i$-th PU has full CSI for the direct channel power gain $g_{i}$ between $\mathrm{PT}_{i}$ and $\mathrm{PR}_{i}$ only.

Remark 2.1.1. In our problem formulation, we do not allow the primary users to share the $N$ channel bands to avoid PUs causing interference to each other. Consideration of PU generated mutual interference or an appropriate scheduling policy that allocates each band to a distinct PU in every fading block will render our problem formulation rather complex and is beyond the scope of our work. Note however that it is easy to extend the results in this chapter to the case where each primary user has a distinct set of subcarriers (so that the primary users do not cause interference to each other) that it can use, although this will result in an increased complexity for the power allocation problem.

Remark 2.1.2. Note that the assumption of full CSI at the ST of all channels is not realistic for a practical cognitive radio system, as much as in any existing wireless communication systems. In particular, obtaining full channel information of the SU-PR channels and PT-PR channels may be difficult. In recent literature however, some practical schemes have been suggested for obtaining such information at the $\mathrm{ST}$ in [43]. For ST-PR channels, it is suggested that the ST can estimate this gain by 
measuring received power of signals transmitted by the $\mathrm{PR}$ and under the assumption of channel reciprocity and that ST knows the PR transmission power. For PT-PR channels, various suggestions have been made including that of eavesdropping on PR feedback to PT [77], and receiving feedback from a cooperative SU node employed near the PR [46], while information about ST-SR channels can be obtained via classical channel feedback and training schemes. Furthermore, power allocation for the secondary user's ergodic capacity maximization under average transmit power and average interference (peak interference) (at the primary receiver) constraints in a spectrum sharing scenario with quantized CSI (or limited feedback) has been investigated in [60]. Design and analysis of such limited feedback based design for the service-outage considered in this current submission is a considerably much harder problem and will be investigated in future work. The results obtained in this chapter based on full CSI will serve as a benchmark for any such future results based on partial or imperfect CSI.

Remark 2.1.3. We have addressed the above issue of possible unavailability of full CSI at the ST further by illustrating the effect of imperfect or partial CSI at the ST in terms of SU ergodic capacity loss. We have carried out a sensitivity analysis with respect to noisy estimated CSI of the PT-PR channels $g_{i}, i=1,2, \ldots, N$. For further details, see Figure 2.6 in Section 2.4.

Our main focus is on the service-outage based power allocation problem which combines the concepts of ergodic capacity and outage capacity. Let $P_{p i}\left(g_{i}\right)$ represent $\mathrm{PU}_{i}$ 's power strategy as $\mathrm{PU}_{i}$ 's power policy is assumed to be determined by the direct channel power gain $g_{i}$ between $\mathrm{PT}_{i}$ and $\mathrm{PR}_{i}$ only, due to a common assumption that the PUs are generally oblivious to the presence of the SU. $\mathbf{P}_{s}(\boldsymbol{\nu})=\left[P_{s 1}(\boldsymbol{\nu}), \ldots, P_{s N}(\boldsymbol{\nu})\right]$ denotes SU's power allocation strategy as a function of the channel state $\boldsymbol{\nu}$, where $P_{s i}(\boldsymbol{\nu})$ is the transmission power for the $i$-th band. The instantaneous transmission rates $r_{p i}$ for the $i$-th PU and $r_{s}$ for the SU can be defined as $r_{p i}\left(\boldsymbol{\nu}, P_{s i}(\boldsymbol{\nu})\right)=\log \left(1+\frac{g_{i} P_{p i}\left(g_{i}\right)}{\beta_{i} P_{s i}(\boldsymbol{\nu})+N_{0}}\right)$ and $r_{s}(\boldsymbol{\nu}, \mathbf{P}(\boldsymbol{\nu}))=\sum_{i=1}^{N} r_{s i}\left(\boldsymbol{\nu}, P_{s i}(\boldsymbol{\nu})\right)=$ $\sum_{i=1}^{N} \log \left(1+\frac{h_{i} P_{s i}(\boldsymbol{\nu})}{\alpha_{i} P_{p i}\left(g_{i}\right)+N_{0}}\right)$. Note that we drop the constant $\frac{1}{2}$ and use natural logarithm for simplicity and $g_{i}$ is in fact an element of $\boldsymbol{\nu}$. Let $r_{p i}^{0}$ and $r_{s}^{0}$ denote the service 
rates of $\mathrm{PU}_{i}$ and the $\mathrm{SU}$, respectively, and $\mathbf{r}_{p}^{0}=\left[r_{p 1}^{0}, r_{p 2}^{0}, \ldots, r_{p N}^{0}\right]$. In this work, we assume that each PU adopts an ON-OFF power control policy, i.e. $P_{p i}\left(g_{i}\right)=P_{c i}$ if $g_{i} \geq g_{T, i}=\frac{\left(e^{r_{p i}^{0}}-1\right) N_{0}}{P_{c i}}$, i.e. $g_{i}$ is good enough to support $r_{p i}^{0}$, and $P_{p i}\left(g_{i}\right)=0$ otherwise. In the absence of any $\mathrm{SU}$ transmission, $\mathrm{PU}_{i}$ 's outage probability becomes $\epsilon_{p i}^{c}=\operatorname{Pr}\left\{\log \left(1+\frac{g_{i} P_{c i}}{N_{0}}\right)<r_{p i}^{0}\right\}$. Therefore, when the $\mathrm{SU}$ is active, $\mathrm{PU}_{i}$ 's communication is protected as long as $\epsilon_{p i}^{c} \leq \operatorname{Pr}\left\{\log \left(1+\frac{g_{i} P_{c i}}{\beta_{i} P_{s i}(\boldsymbol{\nu})+N_{0}}\right)<r_{p i}^{0}\right\} \leq \epsilon_{p i}$, where $\epsilon_{p i}$ is the outage probability threshold of $\mathrm{PU}_{i}$. Define $\boldsymbol{\epsilon}_{p}=\left[\epsilon_{p 1}, \ldots, \epsilon_{p N}\right]$ and let $\epsilon_{s}$ represent the SU outage probability threshold. Note that the results in this chapter can be extended to any other transmission power policy for the primary users as long as this policy is known to the SU.

\subsection{Problem formulation}

The service-outage problem in this chapter can be formulated as follows:

$$
\begin{array}{ll}
\max _{\mathbf{P}_{s}(\boldsymbol{\nu}) \succeq 0} & E\left[\sum_{i=1}^{N} r_{s i}\left(\boldsymbol{\nu}, P_{s i}(\boldsymbol{\nu})\right)\right] \\
\text { s.t. } & (a) E\left[\sum_{i=1}^{N} P_{s i}(\boldsymbol{\nu})\right] \leq P_{a v}, \quad(b) \operatorname{Pr}\left\{\sum_{i=1}^{N} r_{s i}\left(\boldsymbol{\nu}, P_{s i}(\boldsymbol{\nu})\right)<r_{s}^{0}\right\} \leq \epsilon_{s} \\
& (c) \operatorname{Pr}\left\{r_{p i}\left(\boldsymbol{\nu}, P_{s i}(\boldsymbol{\nu})\right)<r_{p i}^{0}\right\} \leq \epsilon_{p i}, \forall i
\end{array}
$$

It was shown in $[21,25]$ that for such outage-based optimization problems, a deterministic power allocation policy is not optimal in general, especially for discrete fading channel distributions. Similar to [25] we show that (2.1) can be solved by using a probabilistic power allocation technique, i.e. by treating $\mathbf{P}_{s}(\boldsymbol{\nu})$ as a probabilistic power allocation scheme with a conditional probability density function $(\mathrm{PDF}) f_{\mathbf{P}_{s} \mid \boldsymbol{\nu}}\left(\mathbf{p}_{s} \mid \boldsymbol{\nu}\right)$, having transmit power $P_{s i}(\boldsymbol{\nu})$ through $i$-th subchannel with conditional PDF $f_{P_{s i} \mid \boldsymbol{\nu}}\left(p_{s i} \mid \boldsymbol{\nu}\right)$. Note also that such a probabilistic power allocation policy helps transform the above optimization problem (2.1) into a standard convex optimization problem following similar lines as in $[25] . \mathbf{p}_{s}(\boldsymbol{\nu})=\left[p_{s 1}(\boldsymbol{\nu}), \ldots, p_{s N}(\boldsymbol{\nu})\right]$ where $p_{s i}(\boldsymbol{\nu})$ indicates a deterministic power allocation policy for the $i$-th subchannel. With the assumptions of ergodicity of the fading channels and perfect CSI at 
the SU transmitter, we have

$$
\begin{aligned}
& E\left[r_{s}\left(\boldsymbol{\nu}, \mathbf{P}_{s}(\boldsymbol{\nu})\right)\right]=\iint r_{s}\left(\boldsymbol{\nu}, \mathbf{p}_{s}(\boldsymbol{\nu})\right) f_{\mathbf{P}_{s} \mid \boldsymbol{\nu}}\left(\mathbf{p}_{s} \mid \boldsymbol{\nu}\right) d \mathbf{p}_{s}(\boldsymbol{\nu}) d F(\boldsymbol{\nu}) \\
& E\left[\left\langle\mathbf{P}_{s}(\boldsymbol{\nu})\right\rangle\right]=\iint\left\langle\mathbf{p}_{s}(\boldsymbol{\nu})\right\rangle f_{\mathbf{P}_{s} \mid \boldsymbol{\nu}}\left(\mathbf{p}_{s} \mid \boldsymbol{\nu}\right) d \mathbf{p}_{s}(\boldsymbol{\nu}) d F(\boldsymbol{\nu}) \\
& \operatorname{Pr}\left\{r_{s}\left(\boldsymbol{\nu}, \mathbf{P}_{s}(\boldsymbol{\nu})\right)<r_{s}^{0}\right\}=\iint 1\left(r_{s}\left(\boldsymbol{\nu}, \mathbf{p}_{s}(\boldsymbol{\nu})\right)<r_{s}^{0}\right) f_{\mathbf{P}_{s} \mid \boldsymbol{\nu}}\left(\mathbf{p}_{s} \mid \boldsymbol{\nu}\right) d \mathbf{p}_{s}(\boldsymbol{\nu}) d F(\boldsymbol{\nu}) \\
& \operatorname{Pr}\left\{r_{p i}\left(\boldsymbol{\nu}, P_{s i}(\boldsymbol{\nu})\right)<r_{p i}^{0}\right\}=\iint 1\left(r_{p i}\left(\boldsymbol{\nu}, p_{s i}(\boldsymbol{\nu})\right)<r_{p i}^{0}\right) f_{P_{s i} \mid \boldsymbol{\nu}}\left(p_{s i} \mid \boldsymbol{\nu}\right) d p_{s i}(\boldsymbol{\nu}) d F(\boldsymbol{\nu})
\end{aligned}
$$

where all conditional PDFs are nonnegative, i.e. $f_{\mathbf{P}_{s} \mid \boldsymbol{\nu}}\left(\mathbf{p}_{s} \mid \boldsymbol{\nu}\right) \geq 0$ and $f_{P_{s i} \mid \boldsymbol{\nu}}\left(p_{s i} \mid \boldsymbol{\nu}\right) \geq$ 0 , and $\int f_{\mathbf{P}_{s} \mid \boldsymbol{\nu}}\left(\mathbf{p}_{s} \mid \boldsymbol{\nu}\right) d \mathbf{p}_{s}(\boldsymbol{\nu})$ and $\int f_{P_{s i} \mid \boldsymbol{\nu}}\left(p_{s i} \mid \boldsymbol{\nu}\right) d p_{s i}(\boldsymbol{\nu})$ are 1 for all $i$.

Adapting a technique similar to the one used in [25], we define the 4 weighting functions and 4 corresponding deterministic power schemes for each of the subchannels as shown in (2.3) and (2.4) below.

$$
\begin{array}{cc}
w_{1 i}(\boldsymbol{\nu})= & \operatorname{Pr}\left\{r_{p i}\left(\boldsymbol{\nu}, P_{s i}(\boldsymbol{\nu})\right) \geq r_{p i}^{0}, \sum_{i=1}^{N} r_{s i}\left(\boldsymbol{\nu}, P_{s i}(\boldsymbol{\nu})\right) \geq r_{s}^{0} \mid \boldsymbol{\nu}\right\} \\
w_{2 i}(\boldsymbol{\nu})= & \operatorname{Pr}\left\{r_{p i}\left(\boldsymbol{\nu}, P_{s i}(\boldsymbol{\nu})\right)<r_{p i}^{0}, \sum_{i=1}^{N} r_{s i}\left(\boldsymbol{\nu}, P_{s i}(\boldsymbol{\nu})\right) \geq r_{s}^{0} \mid \boldsymbol{\nu}\right\} \\
w_{3 i}(\boldsymbol{\nu})= & \operatorname{Pr}\left\{r_{p i}\left(\boldsymbol{\nu}, P_{s i}(\boldsymbol{\nu})\right) \geq r_{p i}^{0}, \sum_{i=1}^{N} r_{s i}\left(\boldsymbol{\nu}, P_{s i}(\boldsymbol{\nu})\right)<r_{s}^{0} \mid \boldsymbol{\nu}\right\} \\
w_{4 i}(\boldsymbol{\nu})= & \operatorname{Pr}\left\{r_{p i}\left(\boldsymbol{\nu}, P_{s i}(\boldsymbol{\nu})\right)<r_{p i}^{0}, \sum_{i=1}^{N} r_{s i}\left(\boldsymbol{\nu}, P_{s i}(\boldsymbol{\nu})\right)<r_{s}^{0} \mid \boldsymbol{\nu}\right\} \\
p_{1 i}(\boldsymbol{\nu})= & E\left[P_{s i}(\boldsymbol{\nu}) \mid r_{p i}\left(\boldsymbol{\nu}, p_{s i}(\boldsymbol{\nu})\right) \geq r_{p i}^{0}, \sum_{i=1}^{N} r_{s i}\left(\boldsymbol{\nu}, p_{s i}(\boldsymbol{\nu})\right) \geq r_{s}^{0}, \boldsymbol{\nu}\right] \\
p_{2 i}(\boldsymbol{\nu})= & E\left[P_{s i}(\boldsymbol{\nu}) \mid r_{p i}\left(\boldsymbol{\nu}, p_{s i}(\boldsymbol{\nu})\right)<r_{p i}^{0}, \sum_{i=1}^{N} r_{s i}\left(\boldsymbol{\nu}, p_{s i}(\boldsymbol{\nu})\right) \geq r_{s}^{0}, \boldsymbol{\nu}\right] \\
p_{3 i}(\boldsymbol{\nu})= & E\left[P_{s i}(\boldsymbol{\nu}) \mid r_{p i}\left(\boldsymbol{\nu}, p_{s i}(\boldsymbol{\nu})\right) \geq r_{p i}^{0}, \sum_{i=1}^{N} r_{s i}\left(\boldsymbol{\nu}, p_{s i}(\boldsymbol{\nu})\right)<r_{s}^{0}, \boldsymbol{\nu}\right] \\
p_{4 i}(\boldsymbol{\nu})= & E\left[P_{s i}(\boldsymbol{\nu}) \mid r_{p i}\left(\boldsymbol{\nu}, p_{s i}(\boldsymbol{\nu})\right)<r_{p i}^{0}, \sum_{i=1}^{N} r_{s i}\left(\boldsymbol{\nu}, p_{s i}(\boldsymbol{\nu})\right)<r_{s}^{0}, \boldsymbol{\nu}\right]
\end{array}
$$

Note that the weighting function $w_{k i}(\boldsymbol{\nu})$ represents the probability of using the deterministic power strategy $p_{k i}(\boldsymbol{\nu})$, which is computed as the expectation of the probabilistic power control $P_{s i}(\boldsymbol{\nu})$ within the set that corresponds to $w_{k i}(\boldsymbol{\nu})$.

Let $w_{a}(\boldsymbol{\nu})=\operatorname{Pr}\left\{\sum_{i=1}^{N} r_{s i}\left(\boldsymbol{\nu}, P_{s i}(\boldsymbol{\nu})\right) \geq r_{s}^{0} \mid \boldsymbol{\nu}\right\}$. It follows that $w_{2 i}(\boldsymbol{\nu})=w_{a}(\boldsymbol{\nu})-$ $w_{1 i}(\boldsymbol{\nu})$ and $w_{4 i}(\boldsymbol{\nu})=1-w_{a}(\boldsymbol{\nu})-w_{3 i}(\boldsymbol{\nu})$ for all $i$. Also, let $X_{w_{k i}^{*}}$ be a Bernoulli random variable which is equal to 1 with probability $w_{k i}^{*}$ and 0 for otherwise. We now have the following Lemma the detailed proof of which can be found in Appendix A.1. 
Essentially, given an arbitrary feasibly policy, one can construct a randomized policy as stated in the Lemma below and show that this policy satisfies all the constraints and achieves an equal or better rate than the arbitrary feasible policy.

Lemma 2.2.1. There exists an optimum solution $\mathbf{P}_{s}^{*}(\boldsymbol{\nu})$ of problem (2.1) of the form $P_{s i}^{*}(\boldsymbol{\nu})=\sum_{k=1}^{4} X_{w_{k i}^{*}}(\boldsymbol{\nu}) p_{k i}^{*}(\boldsymbol{\nu})$ where

$$
\begin{aligned}
& \text { - (1) }\left[\sum_{i=1}^{N} r_{s i}\left(\boldsymbol{\nu}, \frac{w_{1 i}^{*}(\boldsymbol{\nu})}{w_{1 i}^{*}(\boldsymbol{\nu})+w_{2 i}^{*}(\boldsymbol{\nu})} p_{1 i}^{*}(\boldsymbol{\nu})+\frac{w_{2 i}^{*}(\boldsymbol{\nu})}{w_{1 i}^{*}(\boldsymbol{\nu})+w_{2 i}^{*}(\boldsymbol{\nu})} p_{2 i}^{*}(\boldsymbol{\nu})\right)-r_{s}^{0}\right] \geq 0 \text {, } \\
& \text { (2) } w_{1 i}^{*}(\boldsymbol{\nu})\left[\frac{1}{\beta_{i}}\left(\frac{g_{i} P_{p i}\left(g_{i}\right)}{e^{r_{p i}^{0}}-1}-N_{0}\right)^{+}-p_{1 i}^{*}(\boldsymbol{\nu})\right] \\
& +w_{3 i}^{*}(\boldsymbol{\nu})\left[\frac{1}{\beta_{i}}\left(\frac{g_{i} P_{p i}\left(g_{i}\right)}{e^{r_{p i}^{0}}-1}-N_{0}\right)^{+}-p_{3 i}^{*}(\boldsymbol{\nu})\right] \geq 0, \\
& \text { (3) } w_{1 i}^{*}(\boldsymbol{\nu})+w_{2 i}^{*}(\boldsymbol{\nu})=w_{1 j}^{*}(\boldsymbol{\nu})+w_{2 j}^{*}(\boldsymbol{\nu})=w_{a}^{*}(\boldsymbol{\nu}), \quad \text { (4) } \sum_{k=1}^{4} w_{k i}^{*}(\boldsymbol{\nu})=1 \text {, } \\
& \text { (5) } E\left[w_{1 i}^{*}(\boldsymbol{\nu})+w_{2 i}^{*}(\boldsymbol{\nu})\right]=E\left[w_{a}^{*}(\boldsymbol{\nu})\right] \geq 1-\epsilon_{s}, \\
& \text { (6) } E\left[w_{1 i}^{*}(\boldsymbol{\nu})+w_{3 i}^{*}(\boldsymbol{\nu})\right] \geq 1-\epsilon_{p i}, \quad \text { (7) } E\left[\sum_{i=1}^{N} \sum_{k=1}^{4} w_{k i}^{*}(\boldsymbol{\nu}) p_{k i}^{*}(\boldsymbol{\nu})\right] \leq P_{a v}
\end{aligned}
$$

for all $i, j \in\{1,2, \ldots N\}$ and $i \neq j$.

Using Lemma 2.2.1, (2.1) can be rewritten as

$$
\begin{aligned}
& \max _{p_{k i} \geq 0, w_{a}, w_{1 i}, w_{3 i}} E\left[\sum_{i=1}^{N} w_{1 i}(\boldsymbol{\nu}) r_{s i}\left(\boldsymbol{\nu}, p_{1 i}(\boldsymbol{\nu})\right)+\left(w_{a}(\boldsymbol{\nu})-w_{1 i}(\boldsymbol{\nu})\right) r_{s i}\left(\boldsymbol{\nu}, p_{2 i}(\boldsymbol{\nu})\right)\right. \\
&\left.+w_{3 i}(\boldsymbol{\nu}) r_{s i}\left(\boldsymbol{\nu}, p_{3 i}(\boldsymbol{\nu})\right)+\left(1-w_{a}(\boldsymbol{\nu})-w_{3 i}(\boldsymbol{\nu})\right) r_{s i}\left(\boldsymbol{\nu}, p_{4 i}(\boldsymbol{\nu})\right)\right]
\end{aligned}
$$

s.t. $(a) E\left[\sum_{i=1}^{N} w_{1 i}(\boldsymbol{\nu}) p_{1 i}(\boldsymbol{\nu})+\left(w_{a}(\boldsymbol{\nu})-w_{1 i}(\boldsymbol{\nu})\right) p_{2 i}(\boldsymbol{\nu})\right.$

$$
\left.+w_{3 i}(\boldsymbol{\nu}) p_{3 i}(\boldsymbol{\nu})+\left(1-w_{a}(\boldsymbol{\nu})-w_{3 i}(\boldsymbol{\nu})\right) p_{4 i}(\boldsymbol{\nu})\right] \leq P_{a v}
$$

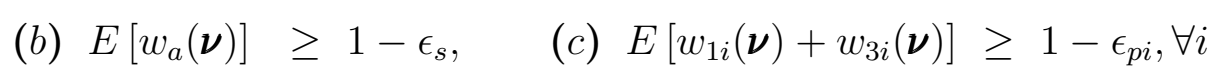

(d) $\left[\sum_{i=1}^{N} r_{s i}\left(\boldsymbol{\nu}, \frac{w_{1 i}(\boldsymbol{\nu})}{w_{a}(\boldsymbol{\nu})} p_{1 i}(\boldsymbol{\nu})+\frac{\left(w_{a}(\boldsymbol{\nu})-w_{1 i}(\boldsymbol{\nu})\right)}{w_{a}(\boldsymbol{\nu})} p_{2 i}(\boldsymbol{\nu})\right)-r_{s}^{0}\right] \geq 0, \forall i$

(e) $w_{1 i}(\boldsymbol{\nu})\left[\frac{1}{\beta_{i}}\left(\frac{g_{i} P_{p i}\left(g_{i}\right)}{e^{r_{p i}^{0}}-1}-N_{0}\right)^{+}-p_{1 i}(\boldsymbol{\nu})\right]+w_{3 i}(\boldsymbol{\nu})\left[\frac{1}{\beta_{i}}\left(\frac{g_{i} P_{p i}\left(g_{i}\right)}{e^{r_{p i}^{0}}-1}-N_{0}\right)^{+}-p_{3 i}(\boldsymbol{\nu})\right] \geq 0, \forall i$

(f) $w_{a}(\boldsymbol{\nu})-w_{1 i}(\boldsymbol{\nu}) \geq 0, \forall i \quad(g) 1-w_{a}(\boldsymbol{\nu})-w_{3 i}(\boldsymbol{\nu}) \geq 0, \forall i$

(h) $w_{1 i}(\boldsymbol{\nu}), w_{3 i}(\boldsymbol{\nu}) \geq 0, \forall i$. 
where $\forall i$ stands for $\forall i=1,2, \ldots, N$. It can be shown that $(2.5)$ is a convex optimization problem. The proof of convexity can be found in Appendix A.2. For convenience, we define $\mathbf{p}_{r s}(\boldsymbol{\nu})=\left[p_{r s, 1}(\boldsymbol{\nu}), \ldots, p_{r s, N}(\boldsymbol{\nu})\right]$ with $p_{r s, i}(\boldsymbol{\nu})=\left(\mu(\boldsymbol{\nu})-\frac{\alpha_{i} P_{p i}\left(g_{i}\right)+N_{0}}{h_{i}}\right)^{+}$ where $\mu(\boldsymbol{\nu})$ satisfies $\sum_{i=1}^{N} r_{s i}\left(\boldsymbol{\nu}, p_{r s, i}(\boldsymbol{\nu})\right)=r_{s}^{0}$, and is derived by minimizing $\sum_{i=1}^{N} p_{r s, i}(\boldsymbol{\nu})$ subject to $\sum_{i=1}^{N} r_{s i}\left(\boldsymbol{\nu}, p_{r s, i}(\boldsymbol{\nu})\right) \geq r_{s}^{0}$. Note that $\mu(\boldsymbol{\nu})$ is obviously strictly positive. Also, define $p_{r p, i}(\boldsymbol{\nu})=\frac{1}{\beta_{i}}\left(\frac{g_{i} P_{p i}\left(g_{i}\right)}{e^{r_{p i}^{0}}-1}-N_{0}\right)^{+}$so that $r_{p i}\left(\boldsymbol{\nu}, p_{r p, i}(\boldsymbol{\nu})\right)=r_{p i}^{0}$.

\subsection{Main results}

In this section, we will focus on solving the optimization Problem (2.1) by a functional optimization technique similar to [25] and conclude with results regarding the feasibility of this problem.

First, note that the Lagrangian for (2.5) can be written as follows

$$
\begin{aligned}
l\left(\boldsymbol{\nu}, p_{k i}(\boldsymbol{\nu}), w_{a}(\boldsymbol{\nu}), w_{1 i}(\boldsymbol{\nu}), w_{3 i}(\boldsymbol{\nu}), \lambda, s_{a}, \mathbf{s}, q(\boldsymbol{\nu}), u_{i}(\boldsymbol{\nu}), \gamma_{i}(\boldsymbol{\nu}), \eta_{i}(\boldsymbol{\nu})\right) \\
=w_{a}(\boldsymbol{\nu})\left[s_{a}+\sum_{i=1}^{N}\left(r_{s i}\left(\boldsymbol{\nu}, p_{2 i}(\boldsymbol{\nu})\right)-\lambda p_{2 i}(\boldsymbol{\nu})+\gamma_{i}(\boldsymbol{\nu})\right)-\sum_{i=1}^{N}\left(r_{s i}\left(\boldsymbol{\nu}, p_{4 i}(\boldsymbol{\nu})\right)-\lambda p_{4 i}(\boldsymbol{\nu})+\eta_{i}(\boldsymbol{\nu})\right)\right]+ \\
\quad \sum_{i=1}^{N} w_{1 i}(\boldsymbol{\nu})\left(s_{i}+r_{s i}\left(\boldsymbol{\nu}, p_{1 i}(\boldsymbol{\nu})\right)-\lambda p_{1 i}(\boldsymbol{\nu})-\left(r_{s i}\left(\boldsymbol{\nu}, p_{2 i}(\boldsymbol{\nu})\right)-\lambda p_{2 i}(\boldsymbol{\nu})\right)-\gamma_{i}(\boldsymbol{\nu})\right)+ \\
\quad \sum_{i=1}^{N} w_{3 i}(\boldsymbol{\nu})\left(s_{i}+r_{s i}\left(\boldsymbol{\nu}, p_{3 i}(\boldsymbol{\nu})\right)-\lambda p_{3 i}(\boldsymbol{\nu})-\left(r_{s i}\left(\boldsymbol{\nu}, p_{4 i}(\boldsymbol{\nu})\right)-\lambda p_{4 i}(\boldsymbol{\nu})\right)-\eta_{i}(\boldsymbol{\nu})\right)+ \\
\quad q(\boldsymbol{\nu})\left(\sum_{i=1}^{N} r_{s i}\left(\boldsymbol{\nu}, \frac{w_{1 i}(\boldsymbol{\nu})}{w_{a}(\boldsymbol{\nu})} p_{1 i}(\boldsymbol{\nu})+\frac{w_{a}(\boldsymbol{\nu})-w_{1 i}(\boldsymbol{\nu})}{w_{a}(\boldsymbol{\nu})} p_{2 i}(\boldsymbol{\nu})\right)-r_{s}^{0}\right)+ \\
\quad \sum_{i=1}^{N} u_{i}(\boldsymbol{\nu})\left(w_{1 i}(\boldsymbol{\nu})\left[p_{r p, i}(\boldsymbol{\nu})-p_{1 i}(\boldsymbol{\nu})\right]+w_{3 i}(\boldsymbol{\nu})\left[p_{r p, i}(\boldsymbol{\nu})-p_{3 i}(\boldsymbol{\nu})\right]\right)
\end{aligned}
$$

where $\lambda, s_{a}, s_{i}, q(\boldsymbol{\nu}), u_{i}(\boldsymbol{\nu}), \gamma_{i}(\boldsymbol{\nu})$, and $\eta_{i}(\boldsymbol{\nu})$ are the nonnegative Lagrange multipliers corresponding to constraints $(2.5 \mathrm{a})$ to $(2.5 \mathrm{~g})$. From the associated (necessary and sufficient) KKT conditions, we have for $i=1,2, \ldots, N$,

$$
\begin{aligned}
& \frac{\partial l(\ldots)}{\partial p_{k i}^{*}(\boldsymbol{\nu})} \begin{cases}=0, & p_{k i}^{*}(\boldsymbol{\nu})>0 \\
\leq 0, & p_{k i}^{*}(\boldsymbol{\nu})=0\end{cases} \\
& \frac{\partial l(\ldots)}{\partial w_{a}^{*}(\boldsymbol{\nu})}=0 \\
& \frac{\partial l(\ldots)}{\partial w_{m i}^{*}(\boldsymbol{\nu})}\left\{\begin{array}{l}
=0, \quad w_{m i}^{*}(\boldsymbol{\nu})>0 \\
\leq 0, \quad w_{m i}^{*}(\boldsymbol{\nu})=0
\end{array} \quad \text {,for } m=1,3\right.
\end{aligned}
$$




$$
\begin{gathered}
\lambda^{*}\left(E \left[\sum_{i=1}^{N} w_{1 i}^{*}(\boldsymbol{\nu}) p_{1 i}^{*}(\boldsymbol{\nu})+\left(w_{a}^{*}(\boldsymbol{\nu})-w_{1 i}^{*}(\boldsymbol{\nu})\right) p_{2 i}^{*}(\boldsymbol{\nu})\right.\right. \\
\left.\left.+w_{3 i}^{*}(\boldsymbol{\nu}) p_{3 i}^{*}(\boldsymbol{\nu})+\left(1-w_{a}^{*}(\boldsymbol{\nu})-w_{3 i}^{*}(\boldsymbol{\nu})\right) p_{4 i}^{*}(\boldsymbol{\nu})\right]-P_{a v}\right)=0 \\
s_{a}^{*}\left(E\left[w_{a}^{*}(\boldsymbol{\nu})\right]-\left(1-\epsilon_{s}\right)\right)=0 \\
s_{i}^{*}\left(E\left[w_{1 i}^{*}(\boldsymbol{\nu})+w_{3 i}^{*}(\boldsymbol{\nu})\right]-\left(1-\epsilon_{p i}\right)\right)=0 \\
q^{*}(\boldsymbol{\nu})\left(\sum_{i=1}^{N} r_{s i}\left(\boldsymbol{\nu}, \frac{w_{1 i}^{*}(\boldsymbol{\nu})}{w_{a}^{*}(\boldsymbol{\nu})} p_{1 i}^{*}(\boldsymbol{\nu})+\frac{\left(w_{a}^{*}(\boldsymbol{\nu})-w_{1 i}^{*}(\boldsymbol{\nu})\right)}{w_{a}^{*}(\boldsymbol{\nu})} p_{2 i}^{*}(\boldsymbol{\nu})\right)-r_{s}^{0}\right)=0 \\
u_{i}^{*}(\boldsymbol{\nu})\left(w_{1 i}^{*}(\boldsymbol{\nu})\left[p_{r p, i}^{*}(\boldsymbol{\nu})-p_{1 i}^{*}(\boldsymbol{\nu})\right]+w_{3 i}^{*}(\boldsymbol{\nu})\left[p_{r p, i}^{*}(\boldsymbol{\nu})-p_{3 i}^{*}(\boldsymbol{\nu})\right]\right)=0 \\
\gamma_{i}^{*}(\boldsymbol{\nu})\left[w_{a}^{*}(\boldsymbol{\nu})-w_{1 i}^{*}(\boldsymbol{\nu})\right]=0 \\
\eta_{i}^{*}(\boldsymbol{\nu})\left[1-w_{a}^{*}(\boldsymbol{\nu})-w_{3 i}^{*}(\boldsymbol{\nu})\right]=0 \\
w_{1 i}^{*}(\boldsymbol{\nu}), w_{3 i}^{*}(\boldsymbol{\nu}) \geq 0 \\
p_{k i}^{*}(\boldsymbol{\nu}) \geq 0, k=1,2,3,4
\end{gathered}
$$

Using the conditions from (2.7) to (2.19), the Lagrangian at the optimal solution for each state $\boldsymbol{\nu}$ must satisfy

$$
\begin{aligned}
& l\left(\boldsymbol{\nu}, p_{k i}^{*}(\boldsymbol{\nu}), w_{a}^{*}(\boldsymbol{\nu}), w_{1 i}^{*}(\boldsymbol{\nu}), w_{3 i}^{*}(\boldsymbol{\nu}), w_{4 i}^{*}(\boldsymbol{\nu}), \lambda^{*}, s_{a}^{*}, \mathbf{s}^{*}, \gamma_{i}^{*}(\boldsymbol{\nu}), \eta_{i}^{*}(\boldsymbol{\nu})\right) \\
&=w_{a}^{*}(\boldsymbol{\nu})\left(\sum_{i=1}^{N}\left[r_{s i}\left(\boldsymbol{\nu}, p_{2 i}^{*}(\boldsymbol{\nu})\right)-\lambda^{*} p_{2 i}^{*}(\boldsymbol{\nu})\right]-\sum_{i=1}^{N}\left[r_{s i}\left(\boldsymbol{\nu}, p_{4 i}^{*}(\boldsymbol{\nu})\right)-\lambda^{*} p_{4 i}^{*}(\boldsymbol{\nu})\right]\right)+ \\
& \sum_{i=1}^{N} w_{1 i}^{*}(\boldsymbol{\nu})\left[\left(r_{s i}\left(\boldsymbol{\nu}, p_{1 i}^{*}(\boldsymbol{\nu})\right)-\lambda^{*} p_{1 i}^{*}(\boldsymbol{\nu})\right)-\left(r_{s i}\left(\boldsymbol{\nu}, p_{2 i}^{*}(\boldsymbol{\nu})\right)-\lambda^{*} p_{2 i}^{*}(\boldsymbol{\nu})\right)\right]+ \\
& \sum_{i=1}^{N} w_{3 i}^{*}(\boldsymbol{\nu})\left[\left(r_{s i}\left(\boldsymbol{\nu}, p_{3 i}^{*}(\boldsymbol{\nu})\right)-\lambda^{*} p_{3 i}^{*}(\boldsymbol{\nu})\right)-\left(r_{s i}\left(\boldsymbol{\nu}, p_{4 i}^{*}(\boldsymbol{\nu})\right)-\lambda^{*} p_{4 i}^{*}(\boldsymbol{\nu})\right)\right]+ \\
& s_{a}^{*} w_{a}^{*}(\boldsymbol{\nu})+\sum_{i=1}^{N} s_{i}^{*}\left[w_{1 i}^{*}(\boldsymbol{\nu})+w_{3 i}^{*}(\boldsymbol{\nu})\right]
\end{aligned}
$$

We now define the "subchannel benefit functions" as follows

$$
\begin{array}{lll}
\mathscr{B}_{1 i}\left(\boldsymbol{\nu}, p_{1 i}^{*}(\boldsymbol{\nu}), \lambda^{*}, s_{i}^{*}\right) & \triangleq \mathscr{B}_{1 i, \boldsymbol{\nu}} \triangleq r_{s i}\left(\boldsymbol{\nu}, p_{1 i}^{*}(\boldsymbol{\nu})\right)-\lambda^{*} p_{1 i}^{*}(\boldsymbol{\nu})+s_{i}^{*} \\
\mathscr{B}_{2 i}\left(\boldsymbol{\nu}, p_{2 i}^{*}(\boldsymbol{\nu}), \lambda^{*}\right) & \triangleq \mathscr{B}_{2 i, \boldsymbol{\nu}} & \triangleq r_{s i}\left(\boldsymbol{\nu}, p_{2 i}^{*}(\boldsymbol{\nu})\right)-\lambda^{*} p_{2 i}^{*}(\boldsymbol{\nu}) \\
\mathscr{B}_{3 i}\left(\boldsymbol{\nu}, p_{3 i}^{*}(\boldsymbol{\nu}), \lambda^{*}, s_{i}^{*}\right) & \triangleq \mathscr{B}_{3 i, \boldsymbol{\nu}} & \triangleq r_{s i}\left(\boldsymbol{\nu}, p_{3 i}^{*}(\boldsymbol{\nu})\right)-\lambda^{*} p_{3 i}^{*}(\boldsymbol{\nu})+s_{i}^{*} \\
\mathscr{B}_{4 i}\left(\boldsymbol{\nu}, p_{4 i}^{*}(\boldsymbol{\nu}), \lambda^{*}\right) & \triangleq \mathscr{B}_{4 i, \boldsymbol{\nu}} & \triangleq r_{s i}\left(\boldsymbol{\nu}, p_{4 i}^{*}(\boldsymbol{\nu})\right)-\lambda^{*} p_{4 i}^{*}(\boldsymbol{\nu})
\end{array}
$$

Also, define $\mathbf{p}_{w f}\left(\boldsymbol{\nu}, \lambda^{*}\right)=\left[p_{w f, 1}\left(\boldsymbol{\nu}, \lambda^{*}\right), \ldots, p_{w f, N}\left(\boldsymbol{\nu}, \lambda^{*}\right)\right]$ where $p_{w f, i}\left(\boldsymbol{\nu}, \lambda^{*}\right)=\left(\frac{1}{\lambda^{*}}-\frac{\alpha_{i} P_{p i}\left(g_{i}\right)+N_{0}}{h_{i}}\right)^{+}$.

With the above definitions and notations, Theorem 2.3.1 below summarizes the optimal solution to Problem (2.1). The detailed proof can be found in in Appendix A.3. 
Theorem 2.3.1. There exists a minimum average power $P_{\min }$ such that if $P_{a v}=$ $P_{\min }$, we have $P_{s i}^{*}(\boldsymbol{\nu})=\left(X_{w_{1 i}^{*}}(\boldsymbol{\nu})+X_{w_{2 i}^{*}}(\boldsymbol{\nu})\right) p_{r s, i}(\boldsymbol{\nu})$. If $P_{a v}>P_{\min }$,

$$
\begin{aligned}
P_{s i}^{*}(\boldsymbol{\nu})= & p_{w f, i}\left(\boldsymbol{\nu}, \lambda^{*}\right)+X_{w_{1 i}^{*}}(\boldsymbol{\nu})\left[\left(p_{r s, i}(\boldsymbol{\nu})-p_{w f, i}\left(\boldsymbol{\nu}, \lambda^{*}\right)\right)^{+}-\left(p_{w f, i}\left(\boldsymbol{\nu}, \lambda^{*}\right)-p_{r p, i}(\boldsymbol{\nu})\right)^{+}\right] \\
& +X_{w_{2 i}^{*}}(\boldsymbol{\nu})\left[\left(p_{r s, i}(\boldsymbol{\nu})-p_{w f, i}\left(\boldsymbol{\nu}, \lambda^{*}\right)\right)^{+}\right]+X_{w_{3 i}^{*}}(\boldsymbol{\nu})\left[-\left(p_{w f, i}\left(\boldsymbol{\nu}, \lambda^{*}\right)-p_{r p, i}(\boldsymbol{\nu})\right)^{+}\right],
\end{aligned}
$$

where

$$
\begin{aligned}
& w_{1 i}^{*}(\boldsymbol{\nu})= \begin{cases}0, & \mathscr{B}_{1 i, \boldsymbol{\nu}} \leq \mathscr{B}_{2 i, \boldsymbol{\nu}} \\
\kappa_{1 i}^{*}(\boldsymbol{\nu}), & \mathscr{B}_{1 i, \boldsymbol{\nu}}=\mathscr{B}_{2 i, \boldsymbol{\nu}} \quad, \quad w_{2 i}^{*}(\boldsymbol{\nu})=w_{a}^{*}(\boldsymbol{\nu})-w_{1 i}^{*}(\boldsymbol{\nu}), \\
w_{a}^{*}(\boldsymbol{\nu}), & \mathscr{B}_{1 i, \boldsymbol{\nu}} \geq \mathscr{B}_{2 i, \boldsymbol{\nu}}\end{cases} \\
& w_{3 i}^{*}(\boldsymbol{\nu})= \begin{cases}0, & \mathscr{B}_{3 i, \boldsymbol{\nu}} \leq \mathscr{B}_{4 i, \boldsymbol{\nu}} \\
\kappa_{3 i}^{*}(\boldsymbol{\nu}), & \mathscr{B}_{3 i, \boldsymbol{\nu}}=\mathscr{B}_{4 i, \boldsymbol{\nu}} \quad, \quad w_{4 i}^{*}(\boldsymbol{\nu})=\left(1-w_{a}^{*}(\boldsymbol{\nu})\right)-w_{3 i}^{*}(\boldsymbol{\nu}), \\
1-w_{a}^{*}(\boldsymbol{\nu}), & \mathscr{B}_{3 i, \boldsymbol{\nu}} \geq \mathscr{B}_{4 i, \boldsymbol{\nu}}\end{cases} \\
& w_{a}^{*}(\boldsymbol{\nu})=\left\{\begin{aligned}
0, \quad s_{a}^{*} & +\sum_{i \in \mathscr{S}_{1}} \mathscr{B}_{1 i, \boldsymbol{\nu}}+\sum_{i \in \mathscr{S}_{2}} \mathscr{B}_{2 i, \boldsymbol{\nu}}-\sum_{i \in \mathscr{S}_{1} \cap \mathscr{S}_{2}} \mathscr{B}_{1 i, \boldsymbol{\nu}} \\
& \leq \sum_{i \in \mathscr{S}_{3}} \mathscr{B}_{3 i, \boldsymbol{\nu}}+\sum_{i \in \mathscr{S}_{4}} \mathscr{B}_{4 i, \boldsymbol{\nu}}-\sum_{i \in \mathscr{S}_{3} \cap \mathscr{S}_{4}} \mathscr{B}_{3 i, \boldsymbol{\nu}} \\
\kappa_{a}^{*}(\boldsymbol{\nu}), s_{a}^{*} & +\sum_{i \in \mathscr{S}_{1}} \mathscr{B}_{1 i, \boldsymbol{\nu}}+\sum_{i \in \mathscr{S}_{2}} \mathscr{B}_{2 i, \boldsymbol{\nu}}-\sum_{i \in \mathscr{S}_{1} \cap \mathscr{S}_{2}} \mathscr{B}_{1 i, \boldsymbol{\nu}} \\
& =\sum_{i \in \mathscr{S}_{3}} \mathscr{B}_{3 i, \boldsymbol{\nu}}+\sum_{i \in \mathscr{S}_{4}} \mathscr{B}_{4 i, \boldsymbol{\nu}}-\sum_{i \in \mathscr{S}_{3} \cap \mathscr{S}_{4}} \mathscr{B}_{3 i, \boldsymbol{\nu}} \\
1, \quad s_{a}^{*} & +\sum_{i \in \mathscr{S}_{1}} \mathscr{B}_{1 i, \boldsymbol{\nu}}+\sum_{i \in \mathscr{S}_{2}} \mathscr{B}_{2 i, \boldsymbol{\nu}}-\sum_{i \in \mathscr{S}_{1} \cap \mathscr{S}_{2}} \mathscr{B}_{1 i, \boldsymbol{\nu}} \\
& \geq \sum_{i \in \mathscr{S}_{3}} \mathscr{B}_{3 i, \boldsymbol{\nu}}+\sum_{i \in \mathscr{S}_{4}} \mathscr{B}_{4 i, \boldsymbol{\nu}}-\sum_{i \in \mathscr{S}_{3} \cap \mathscr{S}_{4}} \mathscr{B}_{3 i, \boldsymbol{\nu}}
\end{aligned}\right.
\end{aligned}
$$

where $\mathscr{S}_{k}$ is the set of subchannel $i$ that use power policy $p_{k i}^{*}, 0<\kappa_{1 i}^{*}(\boldsymbol{\nu})<$ $w_{a}^{*}(\boldsymbol{\nu}), 0<\kappa_{3 i}^{*}(\boldsymbol{\nu})<1-w_{a}^{*}(\boldsymbol{\nu})$, and $0<\kappa_{a}^{*}(\boldsymbol{\nu})<1 . \lambda^{*}, s_{a}^{*}$, and $s_{i}^{*}, \kappa_{1 i}^{*}(\boldsymbol{\nu})$, $\kappa_{3 i}^{*}(\boldsymbol{\nu})$ and $\kappa_{a}^{*}(\boldsymbol{\nu})$ are the solutions to $E\left[\sum_{i=1}^{N} P_{s i}^{*}(\boldsymbol{\nu})\right]=P_{a v}, E\left[w_{a}^{*}(\boldsymbol{\nu})\right] \geq 1-\epsilon_{s}$, and $E\left[w_{1 i}^{*}(\boldsymbol{\nu})+w_{3 i}^{*}(\boldsymbol{\nu})\right] \geq 1-\epsilon_{p i}$ for all $i$, respectively.

\section{Discussions on the optimal power control scheme:}

- $P_{\text {min }}$ denotes the minimum average power required by the SU to make Problem (2.1) feasible. A discussion on the feasibility condition will be provided in Section IV.A.

- For continuous fading channels, $\mathscr{S}_{1} \cap \mathscr{S}_{2}$ and $\mathscr{S}_{3} \cap \mathscr{S}_{4}$ are empty sets since 
$\mathscr{B}_{1 i, \boldsymbol{\nu}}=\mathscr{B}_{2 i, \boldsymbol{\nu}}$ and $\mathscr{B}_{3 i, \boldsymbol{\nu}}=\mathscr{B}_{4 i, \boldsymbol{\nu}}$ can occur with probability of measure zero for every $i$, making the optimal power allocation scheme deterministic, i.e. only one deterministic power strategy is used for each $\boldsymbol{\nu}$. Obviously, there are at most $2^{N}+1$ possible candidate power control policies, i.e. each channel can use $p_{w f, i}$ or $p_{r p, i}$ or all channels use $\mathbf{p}_{r s}$. When $N=1$, this reduces to only 3 possible power policies, as presented in [76]. This raises the question regarding the necessity of using a probabilistic power allocation policy for continuous fading channels. The justification behind using a probabilistic power allocation law as a candidate for an optimal policy is twofold. First and foremost this transforms Problem (2.1) to a convex optimization problem (2.5) which can then easily be solved using the necessary and sufficient KKT conditions. In addition, this makes the optimal power allocation law applicable to general fading distributions which may have discrete components. In the case of continuous fading distributions (as considered in the Numerical results section), the probabilistic power allocation law simplifies to a switching policy amongst a number of deterministic policies (as explained above). In this case, the benefit of using a probabilistic power allocation technique lies in making the original problem convex and thus easily solvable. More details on this can be found in [25] for the non-cognitive setting.

In order to compute the optimal power allocation policy, we first compute $\mathbf{p}_{r s}(\boldsymbol{\nu})$ and $\mathbf{p}_{w f}\left(\boldsymbol{\nu}, \lambda^{*}\right)$ and $p_{r p, i}(\boldsymbol{\nu})$ for every subchannel for a given $\boldsymbol{\nu}$. Then, the possibility of using each of these deterministic schemes is checked, e.g. $\mathbf{p}_{r s}(\boldsymbol{\nu})$ can be used only when $\mathbf{p}_{w f}\left(\boldsymbol{\nu}, \lambda^{*}\right) \prec \mathbf{p}_{r s}(\boldsymbol{\nu})$ for all subchannels and, for the $i$-th subchannel, $p_{r p, i}(\boldsymbol{\nu})$ can be used only when $p_{r p, i}(\boldsymbol{\nu})>p_{w f, i}\left(\boldsymbol{\nu}, \lambda^{*}\right)$. From the remaining deterministic power allocation policies, for a given channel state, SU selects the power policy that results in the largest value of the Lagrangian function (2.20), which can be interpreted as the total benefit for the SU. Essentially, for a price of $\lambda^{*} \sum_{i=1}^{N} P_{s i}^{*}(\boldsymbol{\nu})$, the SU gets a rate $r_{s}\left(\boldsymbol{\nu}, \boldsymbol{P}_{s}^{*}(\boldsymbol{\nu})\right)$ and gains a benefit of $s_{a}^{*}$ if $\mathrm{SU}$ is not in outage and gains a benefit of $s_{i}^{*}$ if the $i$-th PU is not in outage. Note that these power allocation policies are computed (for every channel state) as a function of a fixed set of values of 
the Lagrange multipliers. In order to determine the optimal values of the Lagrange multipliers, one can use either subgradient based iterative methods or other appropriate numerical search methods in order to satisfy the average SU transmit power and the SU and PU outage probability constraints.

Note that for every channel realization, one has to find an optimal policy from a set of $2^{N}+1$ policies, which can be computationally prohibitive to implement in real time if $N$ is large. Thus motivated, we propose a suboptimal power scheme in Section IV.B which reduces the number of candidate power allocation policies to just three for each channel realization.

- Additionally, we emphasize the role of $\lambda^{*}$ in the derived optimal power allocation scheme. When $\lambda^{*}$ is large, implying that the SU has a low power budget $P_{a v}, \mathrm{SU}$ is more likely to adopt the policy $\boldsymbol{P}_{s}^{*}(\boldsymbol{\nu})=\mathbf{p}_{r s}(\boldsymbol{\nu})$ to support its target rate. However, if $\lambda^{*}$ is small corresponding to a high SU $P_{a v}$ budget, it tends to use $p_{r p, i}(\boldsymbol{\nu})$ in each subchannel to avoid causing disruptions to $\mathrm{PU}_{i}$ 's communication. Also, if all POCs and SOC are dropped, $\lambda^{*}$ simply controls the threshold corresponding to the well known water-filling power allocation policy represented by $\mathbf{p}_{w f}(\boldsymbol{\nu})$.

\subsubsection{Feasibility of the service-outage problem}

It can be seen from Theorem 2.3.1 that an average power budget $P_{a v} \geq P_{\min }$ is required for Problem (2.1) to be feasible, i.e. the $\mathrm{SU}$ requires a minimum average power $P_{\min }$ to at least support the service rate while satisfying all the POCs. The feasibility of the problem $(2.1)$ is therefore directly related to the problem of SU outage capacity maximization subject to all $N$ POCs and an average SU transmission power constraint. In order to compute $P_{\min }$, it is therefore necessary to address the optimal power control problem for SU's outage probability (SO) minimization problem subject to all POCs and ATPC.

The SO minimization problem subject to $N$ POCs and SU's ATPC can be ex- 
pressed as follows:

$$
\begin{array}{ll}
\min _{\mathbf{P}_{s}(\boldsymbol{\nu}) \succeq 0} & \operatorname{Pr}\left\{\sum_{i=1}^{N} r_{s i}\left(\boldsymbol{\nu}, P_{s i}(\boldsymbol{\nu})\right)<r_{s}^{0}\right\} \\
\text { s.t. } & (a) E\left[\sum_{i=1}^{N} P_{s i}(\boldsymbol{\nu})\right] \leq P_{a v}, \quad \text { (b) } \operatorname{Pr}\left\{r_{p i}\left(\boldsymbol{\nu}, P_{s i}(\boldsymbol{\nu})\right)<r_{p i}^{0}\right\} \leq \epsilon_{p i}, \forall i
\end{array}
$$

Note that Problem (2.22) can be regarded as an extension of [74] to the $N>1$ case. This problem can also be solved using the probabilistic power allocation technique. By using the definition of $w_{k i}^{*}(\boldsymbol{\nu})$ and $p_{k i}^{*}(\boldsymbol{\nu})$ as shown in (2.3) and (2.4), we can prove the following result shown in Theorem 2.3.2 which presents the optimal power allocation policy for Problem (2.22). The proof is similar to that of Theorem 2.3.1 and is therefore omitted to avoid repetition.

Theorem 2.3.2. The optimal solution for (2.22) is $P_{s i}^{*}(\boldsymbol{\nu})=\left(X_{w_{1 i}^{*}}(\boldsymbol{\nu})+X_{w_{2 i}^{*}}(\boldsymbol{\nu})\right) p_{r s, i}(\boldsymbol{\nu})=$ $X_{w_{a}^{*}}(\boldsymbol{\nu}) p_{r s, i}(\boldsymbol{\nu})$, where

$$
\begin{aligned}
& w_{1 i}^{*}(\boldsymbol{\nu})=\left\{\begin{array}{ll}
w_{a}^{*}(\boldsymbol{\nu}) & , i \in \mathscr{M} \\
0 & , i \notin \mathscr{M}
\end{array}, \quad w_{2 i}^{*}(\boldsymbol{\nu})=w_{a}^{*}(\boldsymbol{\nu})-w_{1 i}^{*}(\boldsymbol{\nu}),\right. \\
& w_{3 i}^{*}(\boldsymbol{\nu})=\left\{\begin{array}{ll}
1-w_{a}^{*}(\boldsymbol{\nu}) & , i \in \mathscr{J} \\
0 & , i \notin \mathscr{J}
\end{array}, \quad w_{4 i}^{*}(\boldsymbol{\nu})=\left(1-w_{a}^{*}(\boldsymbol{\nu})\right)-w_{3 i}^{*}(\boldsymbol{\nu}),\right. \\
& w_{a}^{*}(\boldsymbol{\nu})= \begin{cases}1 & , \sum_{i \in \mathscr{J}} S_{i}^{*} \leq 1-\Lambda^{*} \sum_{i=1}^{N} p_{r s, i}(\boldsymbol{\nu})+\sum_{i \in \mathscr{M}} S_{i}^{*} \\
\kappa_{a o}^{*}(\boldsymbol{\nu}) & , \sum_{i \in \mathscr{J}} S_{i}^{*}=1-\Lambda^{*} \sum_{i=1}^{N} p_{r s, i}(\boldsymbol{\nu})+\sum_{i \in \mathscr{M}} S_{i}^{*} \\
0 & , \sum_{i \in \mathscr{J}} S_{i}^{*} \geq 1-\Lambda^{*} \sum_{i=1}^{N} p_{r s, i}(\boldsymbol{\nu})+\sum_{i \in \mathscr{M}} S_{i}^{*}\end{cases}
\end{aligned}
$$

where $\mathscr{J}=\left\{j \mid r_{p j}(\boldsymbol{\nu}, 0) \geq r_{p j}^{0}\right\}$ and $\mathscr{M}=\left\{m \mid r_{p m}\left(\boldsymbol{\nu}, p_{r s, m}\right) \geq r_{p m}^{0}\right\}$, and $\mathscr{M} \subseteq \mathscr{J}$. $\Lambda^{*}, S_{i}^{*}$ and $\kappa_{a o}^{*}(\boldsymbol{\nu})$ are solutions to the constraints $E\left[\sum_{i=1}^{N} P_{s i}^{*}(\boldsymbol{\nu})\right]=P_{a v}$ and $E\left[w_{1 i}^{*}(\boldsymbol{\nu})+w_{3 i}^{*}(\boldsymbol{\nu})\right] \geq 1-\epsilon_{p i}$ for all $i$.

Table 2.1 reveals the possible non-negative weighting functions $w_{k i}(\boldsymbol{\nu})$ in each subchannel and corresponding power control policies $p_{k i}(\boldsymbol{\nu})$ for a given channel state $\boldsymbol{\nu}$, leading to the final result in Theorem 2.3.2 which shows that there are 2 deterministic power schemes involved in this problem. Either the SU is OFF in order to allow an outage or it is $\mathrm{ON}$ with power policy $\mathbf{p}_{r s}^{*}(\boldsymbol{\nu})$. In other words, the SU decides to 
Table 2.1: Three possible cases in $i$-th subchannel for a given $\boldsymbol{\nu}$

\begin{tabular}{c|ll}
\hline \hline Cases & Power allocation policy & Candidate non-negative $w_{k i}$ \\
\hline$r_{p i}\left(\boldsymbol{\nu}, p_{r s, i}\right) \geq r_{p i}^{0}$ and & $p_{1 i}^{*}=p_{r s, i}$ and $p_{3 i}^{*}=0$ & $w_{1 i}^{*}=w_{a}^{*}, w_{3 i}^{*}=1-w_{a}^{*}$ \\
$r_{p i}(\boldsymbol{\nu}, 0) \geq r_{p i}^{0}$ & & \\
\hline$r_{p i}\left(\boldsymbol{\nu}, p_{r s, i}\right)<r_{p i}^{0}$ and & $p_{2 i}^{*}=p_{r s, i}$ and $p_{3 i}^{*}=0$ & $w_{2 i}^{*}=1-w_{1 i}^{*}=w_{a}^{*}$, \\
$r_{p i}(\boldsymbol{\nu}, 0) \geq r_{p i}^{0}$ & & $w_{3 i}^{*}=1-w_{a}^{*}$ \\
\hline$r_{p i}\left(\boldsymbol{\nu}, p_{r s, i}\right)<r_{p i}^{0}$ and & $p_{2 i}^{*}=p_{r s, i}$ and $p_{4 i}^{*}=0$ & $w_{2 i}^{*}=1-w_{1 i}^{*}=w_{a}^{*}$, \\
$r_{p i}(\boldsymbol{\nu}, 0)<r_{p i}^{0}$ & & $w_{4 i}^{*}=1-w_{a}^{*}$ \\
\hline \hline
\end{tabular}

gain a benefit of $\sum_{i \in \mathscr{J}} S_{i}^{*}$ when it keeps silent and a benefit of $1-\Lambda^{*} \sum_{i=1}^{N} p_{r s, i}(\boldsymbol{\nu})+\sum_{i \in \mathscr{M}} S_{i}^{*}$ when it is active. It is to be noted also that $S_{i}^{*}$ represents the benefit when $\mathrm{PU}_{i}$ is not in outage. It is not hard to show that the solution above can be specialized to the one proposed in [74] when $N=1$ and can also be specialized to the solution of the SO minimization problem without POCs in parallel fading channels [21].

We now go back to the discussion on feasibility of Problem (2.22) and how to compute $P_{\min }$. Clearly, it is directly related to SU's $\epsilon_{s}$-outage capacity which can be computed through the solution to Problem (2.22). Let $C_{\epsilon_{s}}\left(P_{a v}, \mathbf{r}_{p}^{0}, \boldsymbol{\epsilon}_{p}\right)$ stand for SU's $\epsilon_{s}$-outage capacity with POCs given fixed $\mathbf{r}_{p}^{0}, \boldsymbol{\epsilon}_{p}, \epsilon_{s}$, and $P_{a v} . C_{\epsilon_{s}}\left(P_{a v}, \mathbf{r}_{p}^{0}, \boldsymbol{\epsilon}_{p}\right)$ is the maximum instantaneous rate at which the $\mathrm{SU}$ can transmit with an outage probability $\epsilon_{s}$ under POCs. So, problem (2.1) is feasible iff the SU target rate $r_{s}^{0} \leq C_{\epsilon_{s}}\left(P_{a v}, \mathbf{r}_{p}^{0}, \boldsymbol{\epsilon}_{p}\right)$. For simplicity, let $P_{\min }\left(r_{s}^{0}, \mathbf{r}_{p}^{0}, \boldsymbol{\epsilon}_{p}\right)=P_{\min }$ be the minimum SU average power needed to support $r_{s}^{0}$ with outage probability $\epsilon_{s}$ while satisfying all $N$ POCs. Hence, the feasibility condition for our main problem is then $P_{a v} \geq P_{\min }$. If $P_{a v}=P_{\min }$, then $\mathbf{p}_{1}^{*}(\boldsymbol{\nu})=\mathbf{p}_{2}^{*}(\boldsymbol{\nu})=\mathbf{p}_{r s}(\boldsymbol{\nu})$ and $\mathbf{p}_{3}^{*}(\boldsymbol{\nu})=\mathbf{p}_{4}^{*}(\boldsymbol{\nu})=0$ due to Theorem 2.3.2.

To compute $P_{\min }$, we search for the optimal $\Lambda^{*}$ and all $S_{i}^{*}$ that solve $E\left[w_{a}^{*}(\boldsymbol{\nu})\right]=$ $1-\epsilon_{s}, E\left[w_{1 i}^{*}(\boldsymbol{\nu})+w_{3 i}^{*}(\boldsymbol{\nu})\right] \geq 1-\epsilon_{p i}$ for all $i$. Once $\Lambda^{*}$ and $S_{i}^{*}$ are obtained, $P_{\min }$ can be computed as $E\left[\left\langle\mathbf{P}_{s}^{*}\right\rangle\right]$. 


\subsubsection{Proposed suboptimal power control}

The optimal solution to Problem (2.1) shows that the optimal power allocation policy can be evaluated to be one of at most $2^{N}+1$ candidate policies for every channel realization $\boldsymbol{\nu}$, based on the pre-computed Lagrange multipliers $\lambda, s_{a}$, and all $s_{i}$, leading to a high computational complexity when $N$ is large. In this section we will investigate a suboptimal power control policy which can help lower this computational burden. Note that the Lagrange multipliers $\lambda, s_{a}$, and all $s_{i}$ can be computed off-line as they depend on long term average constraints, and thus only on the channel statistics and not on the instantaneous channel values. Below we show that our suboptimal power allocation scheme reduces the number of candidate power control policies from at most $2^{N}+1$ candidate policies to only at most 3 candidate policies.

We first make some useful observations about the optimal power allocation solution presented in Theorem 2.3.1.

- If $s_{a}^{*}=0$, the optimization problem reduces to $N$ separate optimization subproblems coupled by the same $\lambda^{*}$, allowing each subchannel of SU to select a power strategy between $p_{w f, i}(\boldsymbol{\nu})$ and $p_{w f, i}(\boldsymbol{\nu})-\left(p_{w f, i}(\boldsymbol{\nu})-p_{r p, i}(\boldsymbol{\nu})\right)^{+}$independently of the strategies used in other subchannels. Roughly speaking, the SU is always allowed to transmit with $p_{w f, i}$ when $p_{w f, i}<p_{r p, i}$. Otherwise, SU has to decide whether $p_{w f, i}(\boldsymbol{\nu})$ or $p_{r p, i}(\boldsymbol{\nu})$ will return a higher benefit. It is not hard to show that if there exists some $s_{i}^{*}=0$, the corresponding power control in that $i$-th subchannel is $p_{w f, i}$. Furthermore, note that there is no need to use the strategy $\mathbf{p}_{r s}(\boldsymbol{\nu})$ since the SOC is inactive.

- If $s_{a}^{*}>0$ and $s_{i}^{*}=0$ for all $i$, there are just two candidate power strategies which are $\mathbf{p}_{w f}(\boldsymbol{\nu})+\left(\mathbf{p}_{r s}(\boldsymbol{\nu})-\mathbf{p}_{w f}(\boldsymbol{\nu})\right)^{+}$and $\mathbf{p}_{w f}(\boldsymbol{\nu})$. In this case, all POCs are inactive.

- The least complicated circumstance arises when both $s_{a}^{*}$ and all $s_{i}^{*}$ are zero. The optimal power control is just $\mathbf{p}_{w f}(\boldsymbol{\nu})$.

- The most complicated scenario arises when both $s_{a}^{*}$ and all $s_{i}^{*}$ are positive. In 
this case, the SU can either decide to transmit with $\mathbf{p}_{w f}(\boldsymbol{\nu})+\left(\mathbf{p}_{r s}(\boldsymbol{\nu})-\mathbf{p}_{w f}(\boldsymbol{\nu})\right)^{+}$ or select whether to transmit with $p_{w f, i}(\boldsymbol{\nu})$ or with $p_{w f, i}(\boldsymbol{\nu})-\left(p_{w f, i}(\boldsymbol{\nu})-\right.$ $\left.p_{r p, i}(\boldsymbol{\nu})\right)^{+}$in the $i$-th band for a given channel state $\boldsymbol{\nu}$. Thus the SU has to select one strategy out of at most $2^{N}+1$ strategies for every realization $\boldsymbol{\nu}$. Moreover, the tradeoff between satisfying the SU service rate and avoiding causing PU outages makes the optimal power strategy impossible to be selected independently in each subchannel.

The proposed suboptimal power control will therefore focus on the worst case scenario when both $s_{a}^{*}$ and some or all $s_{i}^{*}$ are positive, so that the number of candidate power strategies in each $\boldsymbol{\nu}$ is substantially reduced while sacrificing a small SU ergodic capacity loss. Note that for the first three cases above, the suboptimal power control is kept the same as the optimal one due to its low complexity. However, the remaining power strategies must be selected carefully since the SU must satisfy both SOC and all POCs. Below we describe how we choose the three candidate power vector policies $\mathbf{P}_{1}, \mathbf{P}_{2}$ and $\mathbf{P}_{3}$ ) which constitute the suboptimal power control policies for each $\boldsymbol{\nu}$.

(1): $\mathbf{P}_{1}=\mathbf{p}_{w f}(\boldsymbol{\nu})$ is utilized when SU decides to neglect the outage situation for all PUs and SU itself.

(2): $\mathbf{P}_{2}=\mathbf{p}_{w f}(\boldsymbol{\nu})+\left(\mathbf{p}_{r s}(\boldsymbol{\nu})-\mathbf{p}_{w f}(\boldsymbol{\nu})\right)^{+}$is kept as one of the candidates since it helps SU support its service rate.

(3): For $\mathbf{P}_{3}$, we assign the $i$-th power element to be $P_{3 i}=p_{w f, i}$ for $s_{i}^{*}=0$. For $s_{i}^{*}>0, P_{3 i}=p_{w f, i}(\boldsymbol{\nu})-\left(p_{w f, i}(\boldsymbol{\nu})-p_{r p, i}(\boldsymbol{\nu})\right)^{+}$. Note that this power policy is used to prevent PUs' outage situation in all subchannels (1 power policy) instead of considering all possible candidates that can protect each subchannel (up to $2^{N}$ policies) in the optimal solution.

In our proposed suboptimal power control, the number of candidate power strategies reduces from at most $2^{N}+1$ to just 3 for each $\boldsymbol{\nu}$. Furthermore, it is not hard to show that this suboptimal scheme is in fact optimal when $N=1$. 


\subsection{Numerical results}

In this section, we will illustrate via simulation results the performance of the optimal solutions to the service-outage based capacity optimization problem (2.1), the generalized SO minimization problem (2.22), and also the performance of the suboptimal power allocation policy. All channel power gains are assumed to be mutually independent and exponentially distributed (Rayleigh fading) with unit mean. Noises at all PR and SR for each subchannel are presumed to be equal and AWGN with a normalized unit variance, i.e. $N_{0}=1$. This allows us to consider the SU and PU transmit powers and the SU average transmit power constraint in unitless terms, and thus expressed in $\mathrm{dB}$. Note also that the SU average transmission power can be regarded as the average transmit SNR at the SU transmitter. We assume that all PUs are symmetric with identical transmit power $P_{c i}=15 \mathrm{~dB}$, identical maximum outage probability $\epsilon_{p i}=0.1$, and identical service rates, the exact value of which will be specified within the individual simulation descriptions below. The SU outage probability threshold $\epsilon_{s}$ is also set to be 0.1 . The units of the service rates for $\mathrm{SU}$ and PU are expressed in nats/transmission. In the various figures illustrating the simulation results, $R_{s}$ (in nats/transmission) denotes the SU ergodic capacity achieved under various combinations of primary and secondary outage constraints.

\subsubsection{SO minimization problem}

Fig. 2.1 illustrates the SU outage probability performance of the optimal solution to Problem (2.22) for $N=2$ subchannels with $r_{s}^{0}=0.8, r_{s}^{0}=2$ when $r_{p i}^{0}=$ 0.4. The results for the SO minimization problem without POCs are also included as a lower bound to the solution to Problem (2.22). Noticeably, POCs make the outage probability graph saturate in the high average power region, i.e. the SU cannot reduce its outage probability substantially regardless of how large its average transmission power budget is. Also as expected, the SU can have a lower outage probability when the service rate is lower. For a fixed $r_{s}^{0}=0.8$, Fig. 2.2 illustrates the SU outage probability performance for various values of $r_{p i}^{0}$. Clearly, SU can achieve a lower outage probability when PUs' service rates are decreased. However, 


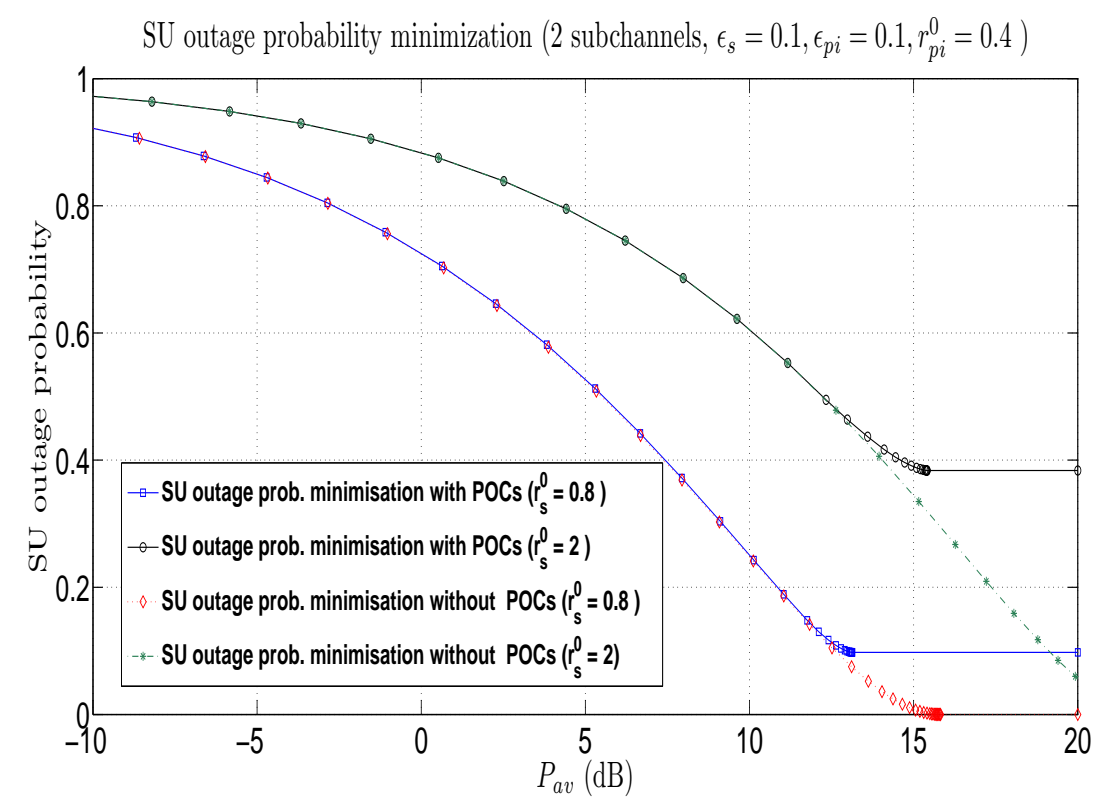

Figure 2.1: SU outage probability performance from SU outage probability minimization problem with POCs with varying $r_{s}^{0}\left(\epsilon_{p i}=0.1, \epsilon_{s}=0.1, r_{p i}^{0}=0.4\right)$

one may wonder why the SU outage probability with higher PUs' target rates are marginally better in the low SNR region (indicated by the green oval). This is due to the ON-OFF PU power control policy. In particular, in the low SNR region, with higher $r_{p i}^{0}, \mathrm{PU}_{i}$ is more likely to turn OFF and thus be in outage since the threshold of $g_{T, i}$ is high. This allows the SU more opportunities to transmit. However, at high SNR, POCs become active and they get stricter with higher $r_{p i}^{0}$, thus restricting the SU transmission opportunities, and increasing its outage probability. In Fig.2.3, we compare the SU $\epsilon_{s}$-outage capacities with POCs and without POCs for $N=$ 4 subchannels. Obviously, the one without POCs will serve as an upper bound of the one with POCs. The results also show that the under the POCs, the SU outage capacity eventually saturate and cannot increase any further, regardless of the average transmission power. This implies that the SU cannot have a service rate $r_{s}^{0}$ to be more than a certain 'maximum target rate', say $r_{s, M A X}^{0}$ when the POCs are present. This observation is important for Problem (2.1) since this problem is feasible iff $P_{a v} \geq P_{\min }$ where $P_{\min }$ is the minimum power that can support $\mathrm{SU} \epsilon_{s^{-}}$ outage capacity $r_{s}^{0}$ when the POCs are present. Hence, if $r_{s}^{0}>r_{s, \text { MAX }}^{0}$, then $P_{\min }$ becomes infinity. 


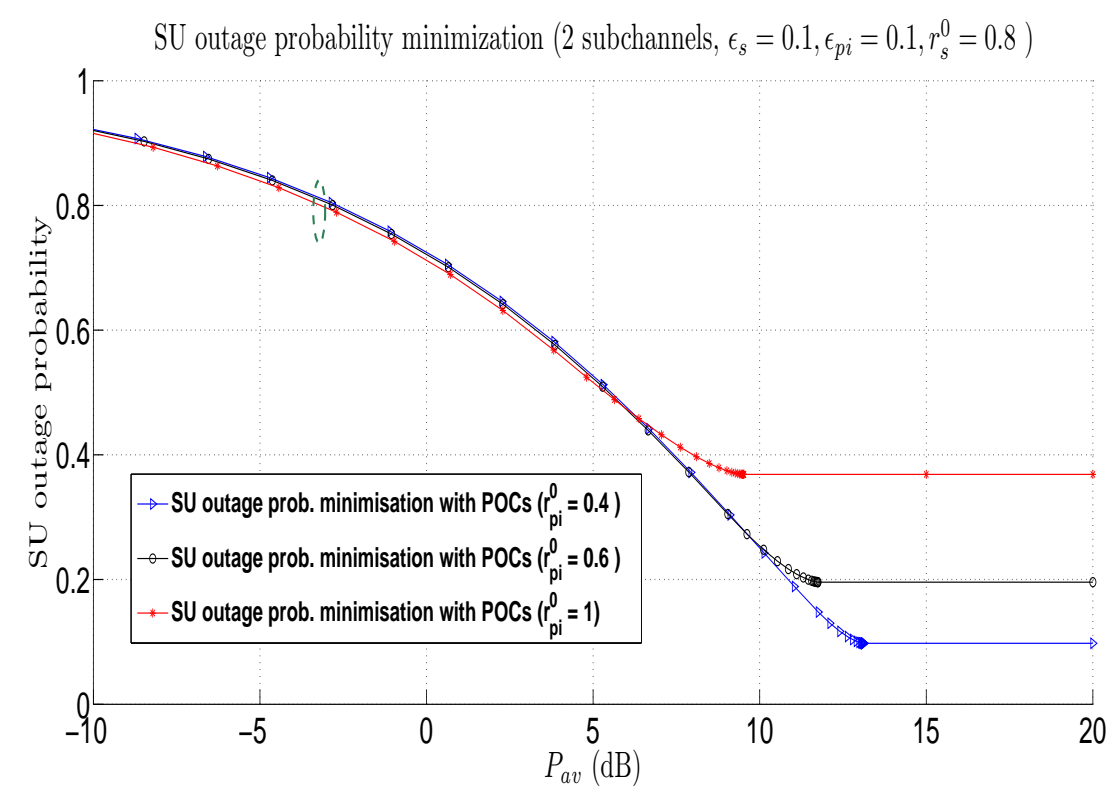

Figure 2.2: SU outage probability performance from SU outage probability minimization problem with POCs with varying $r_{p i}^{0}\left(\epsilon_{p i}=0.1, \epsilon_{s}=0.1, r_{p i}^{0}=0.4\right)$

\subsubsection{SEC maximization problem}

In Fig.2.4, we compare the the SU ergodic capacity achieved by the optimal solution to Problem (2.1) with related bounds for 2 subchannels with $r_{s}^{0}=0.6$ and $r_{p i}^{0}=0.4$. The performance of the optimal solution to Problem (2.1) is denoted by (P1). The optimal SU ergodic capacity performance when the POCs are discarded and only $\mathrm{SOC}$ is present, is represented by (P3), and the SU ergodic capacity performance with the POCs present and SOC discarded is represented by (P2), whereas (P4) represents the $\mathrm{SU} \epsilon_{s}$-outage capacity with all the POCs present. As expected, (P1) and $(\mathrm{P} 4)$ start at the same point $P_{a v}=P_{\min }$, which is the minimum power necessary for feasibility of Problem (2.1). As the available average power $P_{a v}$ increases, the SU ergodic capacity achieved as a solution to Problem (2.1) can enter up to three different stages, as shown in Fig. 2.4. In the first stage, the ergodic capacity graphs (P1) and (P3) are identical since POCs are still inactive. Once at least one POC becomes active (in the second stage), (P1) is upper bounded by both (P3) and (P2). Eventually, as $P_{a v}$ increases even further, (P1) becomes identical with (P2) when the SOC become completely inactive in the third stage. However, it should be clarified that there may not always be a third stage as illustrated by Fig.2.5(a) 


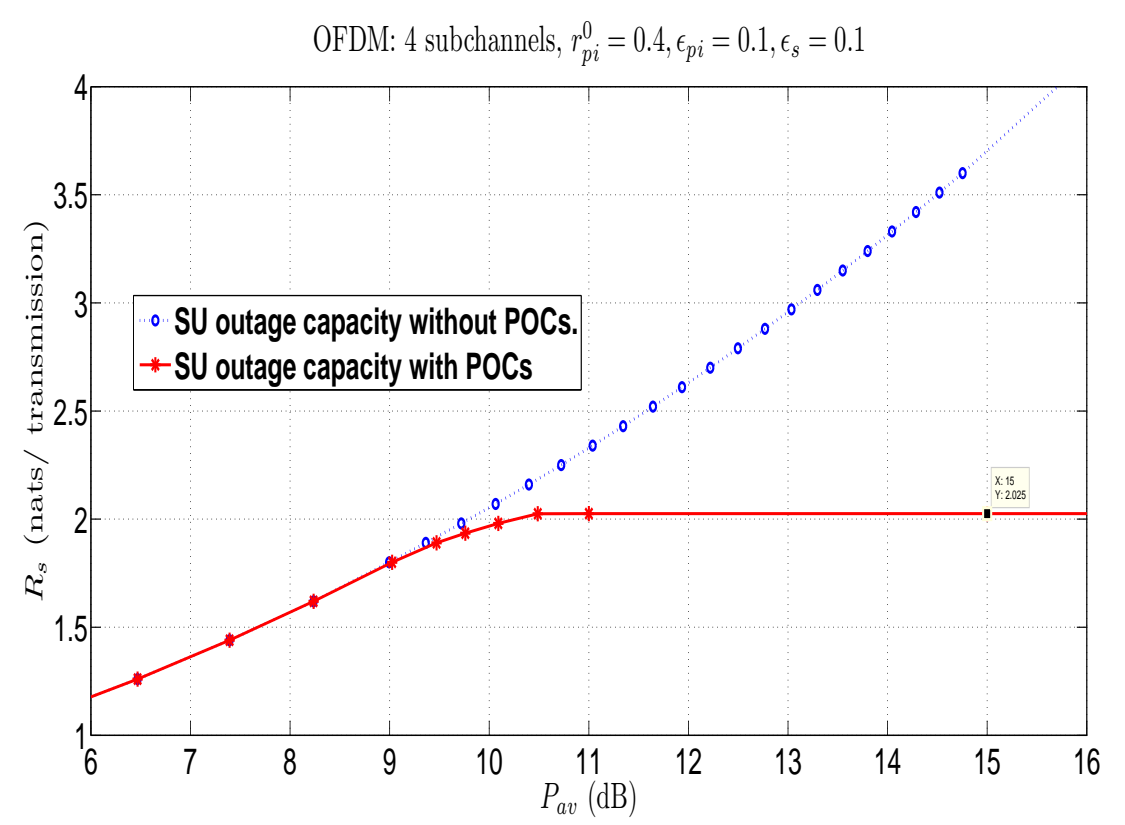

Figure 2.3: SU outage capacity versus average power budget from SU outage probability minimization problem with 4 subchannels $\left(\epsilon_{p i}=0.1, \epsilon_{s}=0.1, r_{p i}^{0}=0.4\right)$

where $r_{s}^{0}$ is varied while all $r_{p i}^{0}$ are still kept at 0.4 . Note that in the third stage, the SU outage constraint becomes inactive, thus, in this stage the SU ergodic capacity results should be independent of $r_{s}^{0}$. As seen in Fig.2.5(a), the SU ergodic capacity graphs of $r_{s}^{0}=0.4$ and $r_{s}^{0}=0.6$ become strictly positive at different $P_{a v}$ (due to different $P_{\min }$ requirements), but they become exactly the same when $P_{a v}$ is high enough which implies that they reach the third stage where SOC becomes inactive. However,this is not the case when $r_{s}^{0}$ is considerably high such that the power strategy $\mathbf{p}_{r s}$ is unavoidable for some channel states. Indeed, when $r_{s}^{0}=0.8$, the SU ergodic capacity does not enter the third stage no matter how high $P_{a v}$ is, as illustrated by Fig.2.5(b), via the performance gap with the SU ergodic capacities for $r_{s}^{0}=0.4$ and $r_{s}^{0}=0.6$. Additionally, in Fig. 2.6, the effect of a noisy estimated channel gain between each $\mathrm{PT}_{i}$ and $\mathrm{PR}_{i}$ on the $\mathrm{SU}$ average rate performance is demonstrated. More specifically, we model the noisy estimated version of $g_{i}$ using the well established model used in $[63,78]$. Let the complex channel amplitude gain between $\mathrm{PU}_{i}$ and SU be $a_{g, i}$. Thus, $g_{i}=\left|a_{g, i}\right|^{2}$, and the noisy estimated version of $a_{g, i}$ is modelled as $\hat{a}_{g, i}=\rho a_{g, i}+\sqrt{1-\rho^{2}} n_{i}$, where $0 \leq \rho \leq 1$ is the correlation coefficient between true and the estimated channel amplitude. $n_{i}$ is the estimation error of $\mathrm{i}$-th 


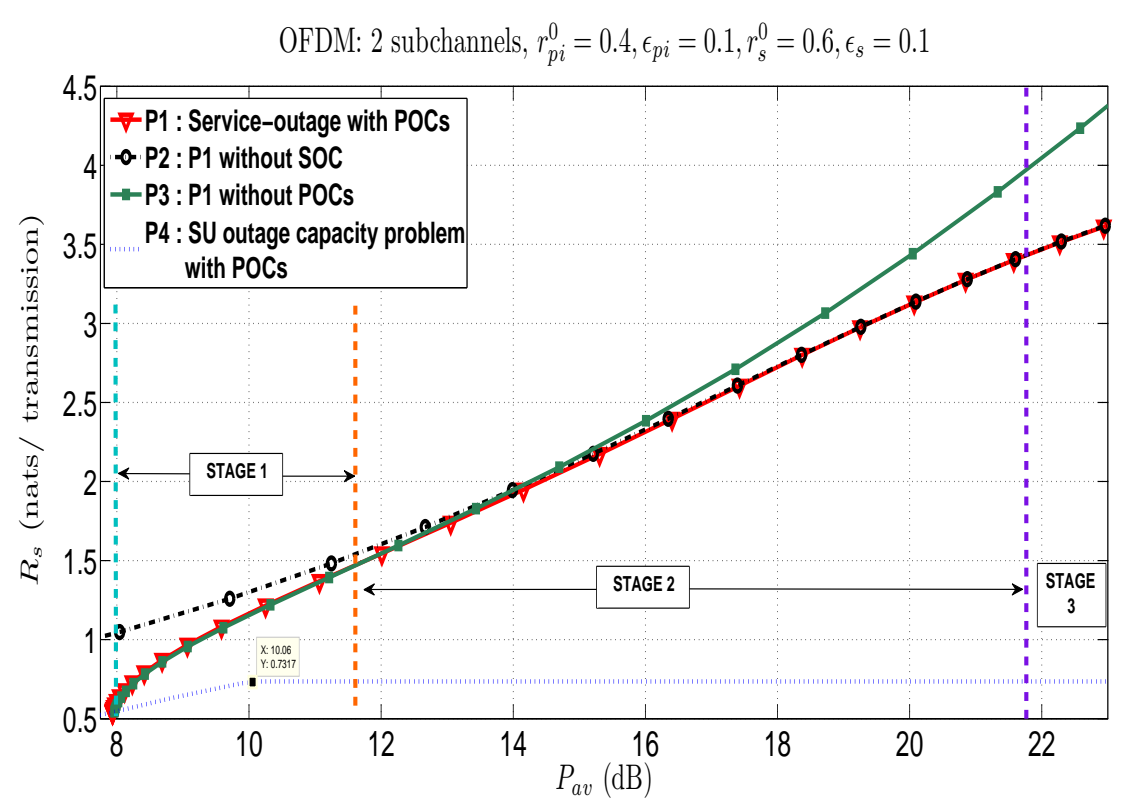

Figure 2.4: Average SU rate performance from SU Ergodic capacity maximization problem with POC and SOC with related bounds $\left(\epsilon_{p i}=0.1, \epsilon_{s}=0.1, r_{p i}^{0}=0.4, r_{s}^{0}=\right.$ $0.6)$

subchannel which is distributed according to a complex normal distribution with zero mean and unit variance. Thus, the estimated channel power gain becomes $\hat{g}_{i}=\left|\hat{a}_{g, i}\right|^{2}$, which has an exponential distribution with unity mean identical to that of $g_{i}$. Figure 2.6 illustrates the effect of $\rho$ on the SU average rate when $N=2$, $r_{s}^{0}=0.6$ nats/transmission, $r_{p i}^{0}=0.4$ nats/transmission for all $i$, and the maximum primary and secondary outage probabilities are 0.1. Note that in designing the power allocation policy, $\hat{g}_{i}$ is used instead of $g_{i}$, but the Lagrange multipliers are chosen such that the PU and SU outage constraints as well as the SU average power constraint are satisfied. Compared to the case of perfect knowledge of $g_{i}$ (i.e. $\rho=1$ ), the average rate loss for the $\mathrm{SU}$ is measured as approximately $6.4925 \%$, $10.1505 \%$ and $20.3530 \%$ for $\rho=0.95,0.9$ and 0.8 , respectively for fixed $P_{a v}=15$ dB. (indicated by blue dashed line). This result indicates that, as $\rho$ decreases, the unreliability in estimated channel gain increases and thus places more restriction on the SU power policy in order to protect QoS in each primary link. Also, the SU requires more average power to make the problem feasible if $\rho$ decreases (indicated by the black dashed oval), since $\hat{g}_{i}$ also affects the term $\frac{h_{i}}{\alpha P_{p}\left(\hat{g}_{i}\right)+N_{0}}$. This results in 
OFDM: 2 subchannels, $r_{p i}^{0}=0.4, \epsilon_{p i}=0.1, \epsilon_{s}=0.1$

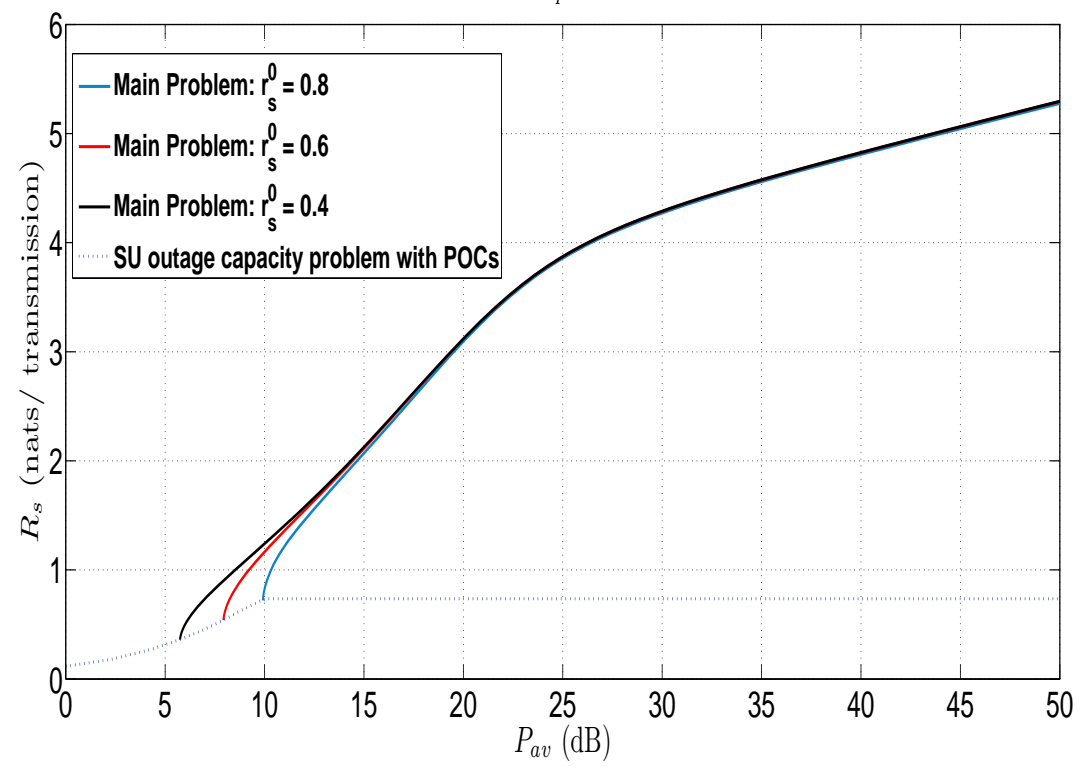

(a)

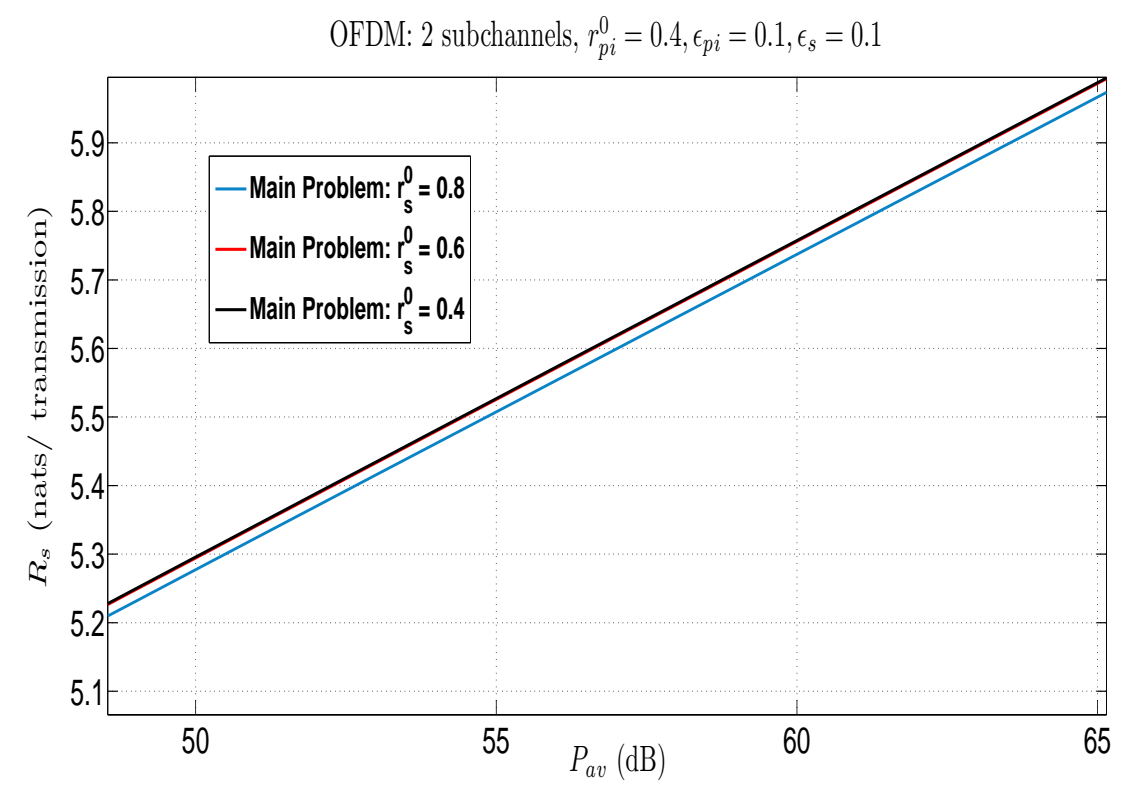

(b) Zooming the area of large $P_{a v}$

Figure 2.5: Average SU rate performance from SU Ergodic capacity maximization problem with POC and SOC with varying $r_{s}^{0}\left(\epsilon_{p i}=0.1, \epsilon_{s}=0.1, r_{p i}^{0}=0.4\right.$ 
the SU misinterpreting the ON-OFF status of $\mathrm{PU}_{i}$ and allocating incorrect power to the individual subchannels.

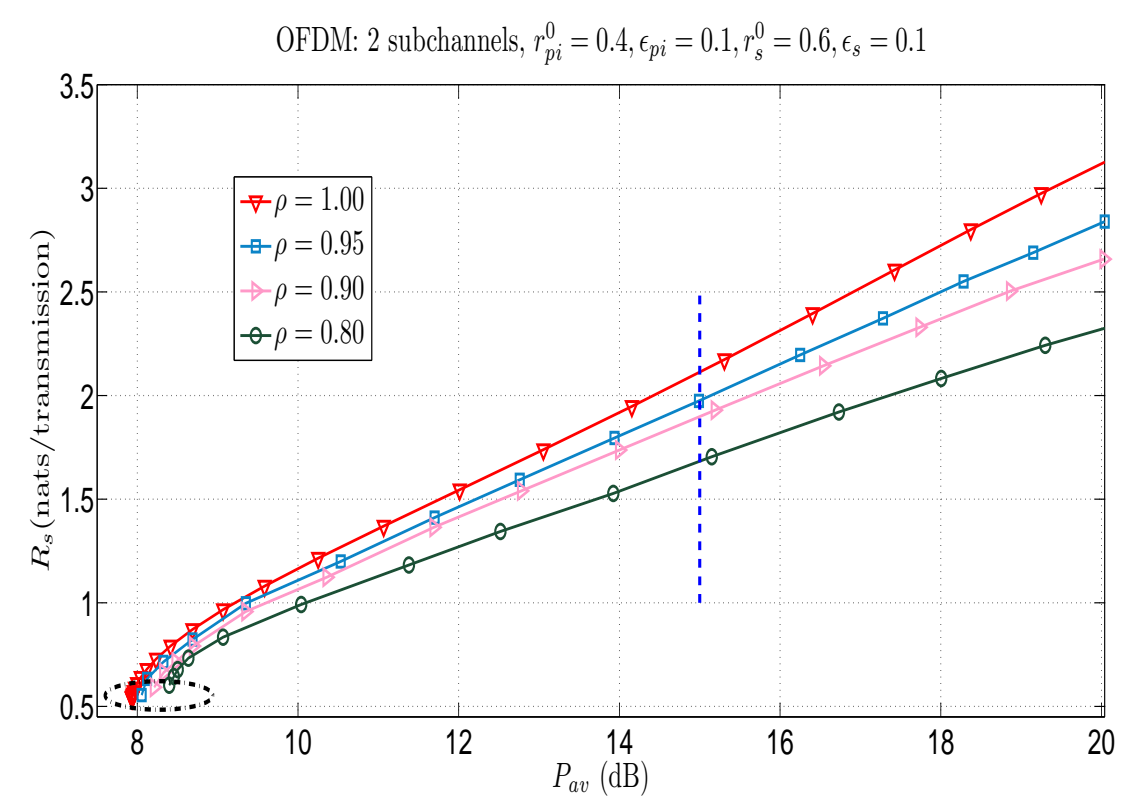

Figure 2.6: The effect of the noisy primary channel estimates on SU ergodic capacity

\subsubsection{Suboptimal power allocation scheme}

In this part we will illustrate the performance of the suboptimal power scheme in comparison with the optimal one. For these simulations, we use $N=16$ subchannels, $r_{s}^{0}=2, r_{p i}^{0}=1.25, \epsilon_{s}=\epsilon_{p i}=0.1$. In Fig. 2.7(a), it is seen that there is a small SU ergodic capacity loss of approximately $5.36 \%$ when the suboptimal algorithm is used, compared to the optimal power control policy, when $s_{a}^{*}$ and all $s_{i}^{*}$ is positive (Stage 2 mentioned above). However, our suboptimal power allocation policy is chosen carefully so that all POC and SOC are ensured, as illustrated in Fig. 2.7(b) for the same example. It implies that the SU can possibly sacrifice a small capacity loss by adopting this suboptimal power strategy, in return for a substantial reduction in real-time computational complexity while still ensuring that SOC and all POCs are satisfied. 


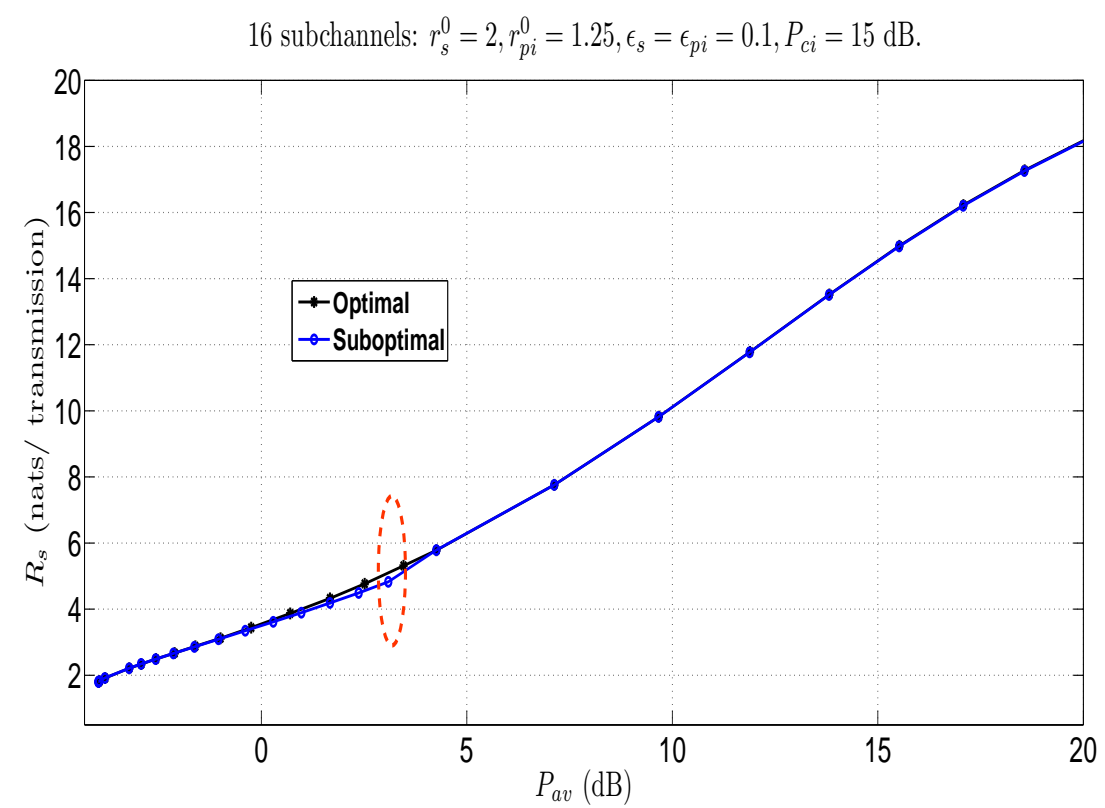

(a) SU capacity

16 subchannels: $r_{s}^{0}=2, r_{p i}^{0}=1.25, \epsilon_{s}=\epsilon_{p i}=0.1, P_{c i}=15 \mathrm{~dB}$.

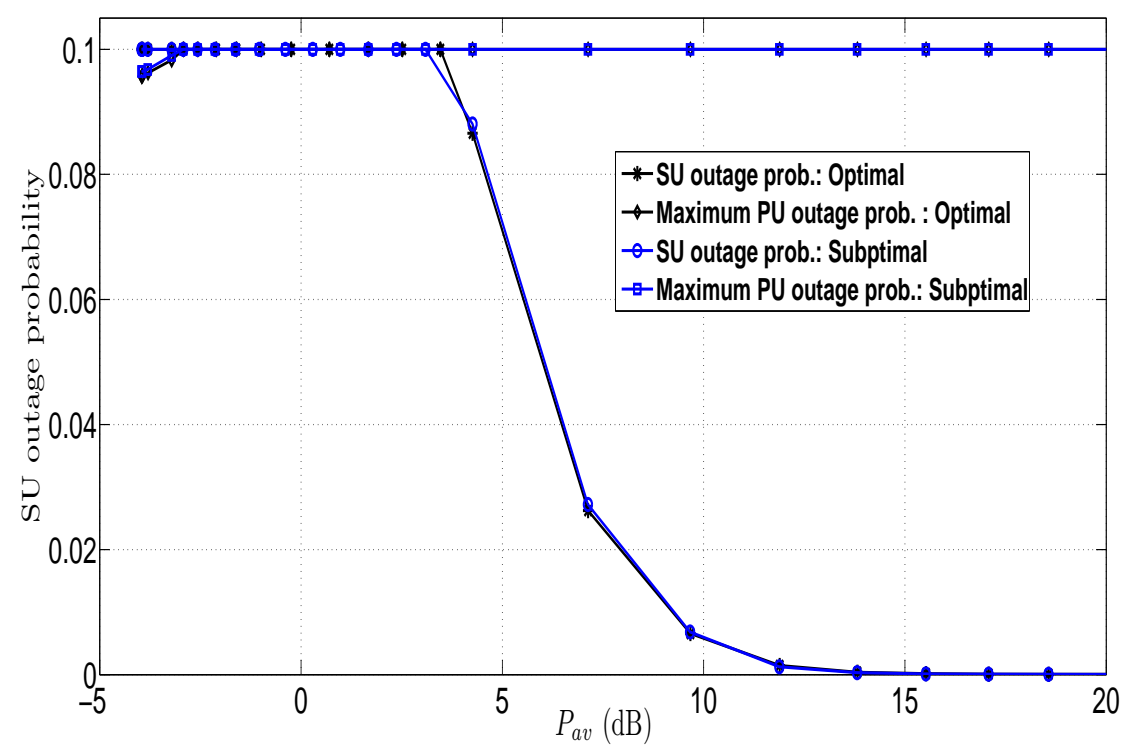

(b) Outage probability

Figure 2.7: Performance comparison between optimal and proposed suboptimal power strategies $(N=16)$ 


\subsection{Conclusion}

This chapter has derived an optimal power allocation policy (under full CSI at the OFDM-based SU transmitter and receiver) in a spectrum sharing cognitive radio network over $N$ parallel fading channels, that maximizes SU ergodic capacity under a SU outage probability constraint, outage probability constraints on all $N$ primary users, and an average transmit power constraint at the SU transmitter. As a special case, we have also solved the secondary outage minimization problem when $N \geq 1$, thus generalizing previous work with $N=1$. The optimal solutions are derived by using a probabilistic power allocation technique that allows our results to be applicable to both continuous and discrete fading channels. To avoid an exponential computational complexity for deriving the SU's optimal power policy in real time for a given channel realization, we have proposed a low-complexity suboptimal power control policy, which substantially reduces the number of computations in each channel realization, while guaranteeing both PU's and SU's outage probability constraints for a small loss in the SU ergodic capacity performance. Numerical results are presented to illustrate the performance of all derived algorithms. 


\section{Chapter 3}

\section{Power Allocation in Cognitive Broadcast Channels with Primary Outage Probability Constraint}

In this chapter, we focus on a single-input single-output (SISO) fading cognitive broadcast channel which co-exists with a delay-sensitive primary link under average and peak transmit power constraint at the secondary base station in underlay cognitive radio paradigm. For non-cognitive SISO fading broadcast channels, information theoretic capacity notions were investigated in, e.g. [15, 16]. In [15], the authors showed that the base station allocates a given time slot (over which the fading channel remains invariant) to the user with the strongest reception only so as to maximize the total throughput, implying that a dynamic time-division-multipleaccess (D-TDMA) is the optimal scheme. Later, the authors of [24] re-emphasized the duality of the capacity region of Gaussian multiple-access channels (MAC) and broadcast channels (BC) by showing that the capacity region of the Gaussian BC under a sum power constraint is exactly the same as the capacity region of a dual Gaussian MAC subject to the same sum power constraint instead of the individual power constraints. Using this result from [24], the authors of [52] investigated the optimal power control for ergodic sum capacity (ESC) maximization in the SISO fading cognitive $\mathrm{BC}$ (C-BC) under both average/peak transmit power constraints and PIPC/AIPC, proving that D-TDMA is the optimal scheme for achieving the ESC in C-BC, reflecting the optimality of the opportunistic scheduling strategy.

Opportunistic user selection strategies have also motivated researchers to analyze how the ESC scales as the number of users $M$ increases. The analysis for throughput scaling in non-cognitive multiple-input multiple-output (MIMO) BC is provided 
in [54]. For underlay cognitive radio networks, there are a number of works studying multiuser diversity. For example, in [79], the authors studied the C-MAC system under a peak transmit power constraint at each secondary transmitter and a peak interference power constraint at the primary receiver and analyzed capacity scaling as the ratio of the transmit power to the interference power approaches infinity. In [80], secondary capacity gains in C-MAC are investigated with the user selection criterion based on the strongest secondary direct channel and the weakest interference channel under a peak interference constraint. Later in [57], the multiuser interference diversity is examined for three types of cognitive networks, including C-MAC, C-BC, and cognitive parallel access channel (C-PAC), under peak transmit power and peak interference power constraints. Recently, the multiuser diversity gain due to optimal power control in C-MAC under average transmit and average interference power constraints with various types of fading channels was investigated in [81] [58]. In contrast to [57] and [58], in this chapter we study the MUD gain under optimal power control for a SISO C-BC under a probabilistic interference constraint for protecting the delay-sensitive primary's transmission, namely, POC.

The novel contributions of this chapter can be summarized as the following:

- We derive the optimal power control policy for the ESC maximization problem in a SISO C-BC under a PU outage probability constraint (POC), under both average transmit power constraint (ATPC) and peak transmit power constraint (PTPC) at the SBS transmitter assuming that perfect knowledge of all involved channel gains at the SBS along with the PU's transmission power control policy. We use a rigorous probabilistic power allocation technique [21, 25, 76] in order to derive the optimal power schemes and we show that for both ATPC and PTPC, a D-TDMA based scheme is optimal when continuous fading channels are considered.

- We derive asymptotic scaling laws for the SBS ergodic sum capacity as the number of SUs, $M \rightarrow \infty$ when SBS uses the optimal power control policy mentioned above, for two types of PU power control policy assuming all channels undergo independent Rayleigh fading: 
1. When the delay-sensitive PU uses an ON-OFF power control policy with a constant power when $\mathrm{ON}$ to meet its basic rate requirement, we show that the SBS ESC scales as $\log \log M$.

2. When the delay-sensitive PU uses a truncated channel inversion (TCI) policy (that minimizes its own outage probability under an average PU transmit power constraint [21] in the absence of the secondary network), we show that the SBS ESC scales according to $\epsilon_{p} \log (\log M)$ with a pre$\log$ factor $\epsilon_{p}, \epsilon_{p}$ being the maximum allowable outage probability at the $\mathrm{PU}$ receiver in presence of the $\mathrm{SU}$ network.

- We also present a set of comprehensive numerical results illustrating our claims on the asymptotic throughput scaling laws as well as the effect of the various parameters involved in the optimization problems.

The remainder of Chapter 3 is organized as follows. The description of our system model is presented in Section 3.1. Under the assumption of full channel side information at SBS and PU's power strategy being known to SBS, the optimal power control policies for the problem with ATPC and PTPC are presented in Section 3.2. When all pertinent channels undergo independent Rayleigh fading, Section 3.3 provides the asymptotic SBS sum throughput scaling results under the optimal power strategy subject to either ATPC or PTPC, given PU's power policy is ON-OFF with a constant power when ON, showing that the SBS sum throughput grows as $\log (\log M)$. In Section 3.4, the asymptotic SBS sum throughput scaling law of $\epsilon_{p} \log (\log M)$ is derived under Rayleigh fading and a TCI power control policy at the PU transmitter and the optimal power control policy at the SBS with both ATPC and PTPC. Table 3.1 summarizes the contributions in Section 3.3 and Section 3.4. The numerical results are presented in Section 3.5 followed by some concluding remarks in Section 3.6.

List of notations in Chapter 3 : Here is a list of important notations used in this chapter. $E[$.$] denotes the statistical expectation. \operatorname{Pr}\{$.$\} represents probability.$ The cumulative density function $(\mathrm{CDF})$ of a random variable $Z$ is given by $F_{Z}(z)$ whereas $F_{Z}(z \mid Y)$ expresses the conditional CDF of $Z$ given $Y$. Let $X_{w}$ be a Bernoulli 
Table 3.1: Throughput analyses in Chapter 3

\begin{tabular}{c|cc|c}
\hline \hline Section & PU's power policy & SU power constraint & Throuput scaling result \\
\hline 3.3 .1 & ON-OFF & ATPC & $\log (\log M)$ \\
\hline 3.3 .2 & ON-OFF & PTPC & $\log (\log M)$ \\
\hline 3.4 .1 & TCI & ATPC & $\epsilon_{p} \log (\log M)$ \\
\hline 3.4 .2 & TCI & PTPC & $\epsilon_{p} \log (\log M)$ \\
\hline \hline
\end{tabular}

random variable such that $X_{w}=1$ with probability $w$ and $X_{w}=0$ with probability $1-w \cdot \frac{\partial y}{\partial x^{*}}$ denotes the partial derivative of $y$ with respect to $x$, evaluated at $x=$ $x^{*} . \quad \mathbf{p}^{T}$ represents the transpose of vector $\mathbf{p} . \quad \mathcal{S}^{c}$ represents the complement of the set $\mathcal{S}$. We also use the notation $f(x)=O(g(x))$ as $x \rightarrow \infty$ to imply that $\lim \sup _{x \rightarrow \infty}\left|\frac{f(x)}{g(x)}\right|<\infty, f(x)=o(g(x))$ as $x \rightarrow \infty$ to imply that $\lim _{x \rightarrow \infty}\left|\frac{f(x)}{g(x)}\right|=0$ and $f(x)=\Theta(g(x))$ as $x \rightarrow \infty$ to imply that there exist positive constants $K_{1}$ and $K_{2}$ such that $K_{1}<\lim _{x \rightarrow \infty}\left|\frac{f(x)}{g(x)}\right|<K_{2}$.

\subsection{System model}

We consider a cognitive fading broadcast channel (C-BC) with one secondary base station (SBS) transmitting to $M$ secondary receivers (SRs), sharing the same spectrum as a primary transmitter-receiver pair (PT-PR). All terminals involved are equipped with a single antenna. All channels involved in this cognitive radio network are assumed to be mutually independent block fading additive white Gaussian noise (BF-AWGN) channels [21] with continuous CDFs. Let the channel gains from SBS to the $i$-th SR, PT to PR, PT to the $i$-th SR, and SBS to PT be denoted by $h_{i}, g, \alpha_{i}$, and $\beta$, respectively, as illustrated in Fig.3.1. Let $\chi$ represent the combined channel state vector, i.e. $\chi=\left\{g, \beta, h_{1}, \ldots, h_{M}, \alpha_{1}, \ldots, \alpha_{M}\right\}$. As usual, we assume the primary user's transmit power control strategy is based only on the direct gain $g$ between PT and PR, regardless of the interference from the secondary network. We also assume that SBS has perfect CSI on $\chi$ and primary user's power policy, so 
that it also knows the PU's power allocation for every realization of $\boldsymbol{\chi}$.

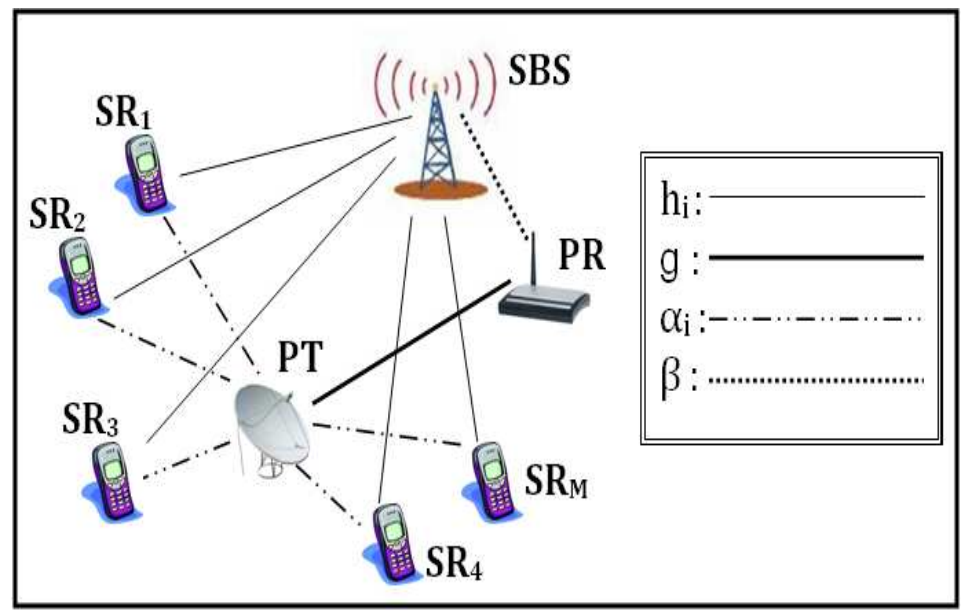

Figure 3.1: System model for cognitive BC

In this chapter, we assume that the QoS guarantee of the delay-sensitive PU with a target rate $r_{p}^{0}$ allows a maximum primary outage probability of $\epsilon_{p}$. In a typical wireless fading environment, even in the absence of interference from the secondary network, the PU may not be able to avoid an outage event when the PTPR channel is in deep fade. For example, with an average or peak transmit power constraint, the PU cannot meet the target rate for a Rayleigh fading channel if it falls below a certain threshold [21] unless it is equipped with multiple antennas. We further presume that the PU's power policy is designed to allow for a maximum PU outage probability of $\epsilon_{p}^{0} \leq \epsilon_{p}$ in the absence of the secondary interference. In this chapter, we assume that the PU employs either an ON-OFF power strategy with a constant power when $O N(O N-O F F)$ or a truncated channel inversion (TCI) power strategy. For the ON-OFF power strategy, the PU transmits with a constant power $P_{p}(g)=P_{c}$ when $g \geq g_{T}=\frac{\left(e^{r_{p}^{0}}-1\right) N_{0}}{P_{c}}$ to meet the target rate and $P_{p}(g)=0$ for $g<g_{T}$, where $N_{0}$ denotes the AWGN variance at the primary receiver. For the TCI power control policy, the amount of the PU's transmit power adapts according to the direct channel gain $g$ when the PU is ON, i.e. $P_{p}(g)=\frac{\left(e^{r_{p}^{0}}-1\right) N_{0}}{g}$ when $g \geq g_{T}$ and $P_{p}(g)=0$ otherwise. Note that [21] has shown that the above TCI power policy is the solution to the primary outage probability minimization problem subject to 
an average transmit power constraint. Note also that $g_{T}$ is chosen such that for $\operatorname{Pr}\left(g<g_{T}\right)=\epsilon_{p}^{0}$. It is worth noting that these two PU power policies are designed based on the PU's direct channel gain $g$ only, reflecting the obliviousness of the PU to the interference from the secondary network.

Let $\mathbf{P}(\boldsymbol{\chi})=\left[P_{1}(\boldsymbol{\chi}), \ldots, P_{M}(\boldsymbol{\chi})\right]^{T}$ where $P_{i}(\boldsymbol{\chi})$ denote the SBS's transmit power allocated for the $i$-th SU receiver. The PU's instantaneous rate expression can be written as

$$
r_{p}(\boldsymbol{\chi}, \mathbf{P}(\boldsymbol{\chi}))=\log \left(1+\frac{g P_{p}(g)}{\left(\beta \sum_{i=1}^{M} P_{i}(\boldsymbol{\chi})\right)+N_{0}}\right)
$$

The delay-sensitive primary network has an outage probability constraint (POC) with a target rate $r_{p}^{0}$ and a maximum outage probability threshold $\epsilon_{p}$, such that

$$
\operatorname{Pr}\left\{r_{p}(\chi, \mathbf{P}(\chi))<r_{p}^{0}\right\} \leq \epsilon_{p}
$$

The power budget at the SBS can be either ATPC or PTPC as shown in (3.3) and (3.4), respectively.

$$
\begin{gathered}
E\left[\sum_{i=1}^{M} P_{i}(\chi)\right] \leq P_{a v} \\
\sum_{i=1}^{M} P_{i}(\chi) \leq P_{O}
\end{gathered}
$$

In this chapter, we focus on solving the ergodic sum capacity maximizing problem in the secondary downlink subject to (3.2) and either (3.3) or (3.4). Similar to [52], by applying the MAC-BC duality result in [24], the capacity region of the C-BC can be written in terms of the capacity region of the dual MAC under an average or peak sum power constraint. Thus, the ergodic sum capacity achieved by the secondary network in this problem can be written as follows

$$
C_{s}=\max _{\mathbf{P}(\chi) \succeq 0} E\left[r_{s}(\boldsymbol{\chi}, \mathbf{P}(\boldsymbol{\chi}))\right] \quad \text { s.t. (3.2) and either (3.3) or (3.4) }
$$

where $r_{s}(\chi, \mathbf{P}(\chi))$ is the instantaneous sum rate of the auxiliary cognitive MAC 
expressed as

$$
r_{s}(\chi, \mathbf{P}(\chi))=\quad \log \left(1+\sum_{i=1}^{M} \frac{h_{i}}{\alpha_{i} P_{p}(g)+N_{0}} P_{i}(\boldsymbol{\chi})\right)
$$

Note that we drop the constant $\frac{1}{2}$ in the instantaneous rate expressions in (3.1) and (3.5) and use natural logarithm for simplicity. We also assume that the AWGN variances in each $\mathrm{SU}$ receiver is given by $N_{0}$ as well.

\subsection{Optimal power strategies}

This section will derive the optimal power schemes such that maximize ergodic sum downlink capacity subject to a POC with either an ATPC in Section 3.2.1 or a PTPC in Section 3.2.2.

The ergodic sum capacity maximization problem from the SBS to $M$ SUs with a POC and an ATPC, (P1), is defined as follows.

$$
\begin{array}{ll}
\text { (P1) } \max _{\mathbf{P}(\boldsymbol{\chi})} & E\left[r_{s}(\boldsymbol{\chi}, \mathbf{P}(\boldsymbol{\chi}))\right] \\
\text { s.t. } & \operatorname{Pr}\left\{r_{p}(\boldsymbol{\chi}, \mathbf{P}(\boldsymbol{\chi}))<r_{p}^{0}\right\} \leq \epsilon_{p} \\
& E\left[\sum_{i=1}^{M} P_{i}(\boldsymbol{\chi})\right] \leq P_{a v} \\
& \mathbf{P}(\boldsymbol{\chi}) \succeq 0
\end{array}
$$

If the constraint (3.6c) is replaced by $\sum_{i=1}^{M} P_{i}(\boldsymbol{\chi}) \leq P_{O}$, then we have an ergodic sum capacity maximization problem with a POC and a PTPC, labelled by (P2). By applying the same technique as in $[21,25,76]$, we can prove that the optimal power control for (P1) and (P2) is randomized between two deterministic schemes, i.e. $\mathbf{p}_{1}(\boldsymbol{\chi})=E\left[\mathbf{P}(\boldsymbol{\chi}) \mid r_{p}(\boldsymbol{\chi}, \mathbf{p}(\boldsymbol{\chi})) \geq r_{p}^{0}\right]$ and $\mathbf{p}_{2}(\boldsymbol{\chi})=E\left[\mathbf{P}(\boldsymbol{\chi}) \mid r_{p}(\boldsymbol{\chi}, \mathbf{p}(\boldsymbol{\chi}))<r_{p}^{0}\right]$, with the probability indicated by the weighting function $w(\chi)$ can be expressed as $w(\boldsymbol{\chi})=\operatorname{Pr}\left\{r_{p}(\boldsymbol{\chi}, \mathbf{P}(\boldsymbol{\chi})) \geq r_{p}^{0} \mid \boldsymbol{\chi}\right\}$. 


\subsubsection{Optimal power policy for ATPC}

Lemma 3.2.1. The optimal solution of the problem (P1) can be expressed by $\mathbf{P}^{*}(\boldsymbol{\chi})=w(\boldsymbol{\chi}) \mathbf{p}_{1}(\boldsymbol{\chi})+(1-w(\boldsymbol{\chi})) \mathbf{p}_{2}(\boldsymbol{\chi})$, where $E[w(\boldsymbol{\chi})] \geq 1-\epsilon_{p}, E\left[\sum_{i=1}^{M} P_{i}^{*}(\boldsymbol{\chi})\right]$ and $r_{p}\left(\chi, \mathbf{p}_{1}(\chi)\right) \geq r_{p}^{0}$ for all $\chi$.

The proof of Lemma 3.2.1 can be found in Appendix B.1. For convenience, we further define $\mathscr{P}_{p}(g)=\left(\frac{g P_{p}(g)}{e^{r} r_{p}^{0}}-N_{0}\right)^{+}$. Reformulating $(\mathbf{P 1})$ by Lemma 3.2.1, we obtain

$$
\begin{array}{ll}
(\mathbf{P 1}) \max _{\mathbf{p}_{k}(\boldsymbol{\chi}), w(\boldsymbol{\chi})} & E\left[w(\boldsymbol{\chi}) r_{s}\left(\boldsymbol{\chi}, \mathbf{p}_{1}(\boldsymbol{\chi})\right)+(1-w(\boldsymbol{\chi})) r_{s}\left(\boldsymbol{\chi}, \mathbf{p}_{2}(\boldsymbol{\chi})\right)\right] \\
\text { s.t. } & E\left[\mathbf{1}^{T}\left(w(\boldsymbol{\chi}) \mathbf{p}_{1}(\boldsymbol{\chi})+(1-w(\boldsymbol{\chi})) \mathbf{p}_{2}(\boldsymbol{\chi})\right)\right] \leq P_{a v} \\
& E[w(\boldsymbol{\chi})] \geq 1-\epsilon_{p} \\
& w(\boldsymbol{\chi})\left[\mathscr{P}_{p}(g)-\beta \mathbf{1}^{T} \mathbf{p}_{1}(\boldsymbol{\chi})\right] \geq 0 \\
& \mathbf{p}_{k}(\boldsymbol{\chi}) \succeq 0, \quad \forall k \in\{1,2\} \\
& 0 \leq w(\boldsymbol{\chi}) \leq 1
\end{array}
$$

In a similar manner as in $[25,76]$, the objective function can be proved to be concave while the other constraints are linear. Hence, the problem $(\mathbf{P 1})$ can be solved by the necessary and sufficient Karush-Kuhn-Tucker (KKT) optimality conditions which are provided in Appendix B.2. For convenience, define $z_{i}=\frac{h_{i}}{\alpha_{i} P_{p}(g)+N_{0}}$ for all $i$, $p_{W F, i}^{*}(\chi)=\left(\frac{1}{\Lambda^{*}}-\frac{1}{z_{i}}\right)^{+}$and $p_{R P}^{*}(\chi)=\frac{\mathscr{P}_{p}(g)}{\beta}$. Applying KKT conditions and the fact that channel state is continuous, the optimal power policy can be summarized in Theorem 3.2.1

Theorem 3.2.1. The optimal power control for $(\boldsymbol{P} \mathbf{1})$ is $\mathbf{P}^{*}=X_{w^{*}}(\boldsymbol{\chi}) \mathbf{p}_{1}^{*}(\boldsymbol{\chi})+$ $\left(1-X_{w^{*}}(\chi)\right) \mathbf{p}_{2}^{*}(\chi)$, where $\lambda^{*}$ and $S^{*}$ are the solutions to $E\left[\sum_{i=1}^{M} P_{i}^{*}(\chi)\right]=P_{a v}$ and $E\left[w^{*}(\chi)\right] \geq 1-\epsilon_{p}$ and $\mathbf{p}_{1}^{*}(\boldsymbol{\chi}), \mathbf{p}_{2}^{*}(\boldsymbol{\chi})$ and $w^{*}(\boldsymbol{\chi})$ are defined as follows

$$
p_{1 i}^{*}(\boldsymbol{\chi})= \begin{cases}p_{W F, i}^{*}(\chi) & , w^{*}(\boldsymbol{\chi})=1, p_{W F, i}^{*}(\boldsymbol{\chi}) \leq p_{R P}^{*}(\boldsymbol{\chi}), i=\arg \max _{m \in \mathcal{I}} z_{m} \\ p_{R P}^{*}(\boldsymbol{\chi}) & , w^{*}(\boldsymbol{\chi})=1, p_{W F, i}^{*}(\boldsymbol{\chi})>p_{R P}^{*}(\boldsymbol{\chi}), i=\arg \max _{m \in \mathcal{I}} z_{m} \\ 0 & , \text { otherwise }\end{cases}
$$




$$
\begin{gathered}
p_{2 i}^{*}(\boldsymbol{\chi})= \begin{cases}p_{W F, i}^{*}(\boldsymbol{\chi}) & , w^{*}(\boldsymbol{\chi})=0, i=\arg \max _{m \in \mathcal{I}} z_{m} \\
0 & , \text { otherwise }\end{cases} \\
w^{*}(\boldsymbol{\chi})= \begin{cases}1, & \mathscr{B}_{1, \boldsymbol{\chi}}^{B}>\mathscr{B}_{2, \boldsymbol{\chi}}^{B} \\
0, & \mathscr{B}_{1, \boldsymbol{\chi}}^{B}<\mathscr{B}_{2, \boldsymbol{\chi}}^{B}\end{cases}
\end{gathered}
$$

where $\mathscr{B}_{1, \boldsymbol{\chi}}^{B}=r_{s}\left(\boldsymbol{\chi}, \mathbf{p}_{1}^{*}(\boldsymbol{\chi})\right)-\Lambda^{*} \mathbf{1}^{T} \mathbf{p}_{1}^{*}(\boldsymbol{\chi})+S^{*}$ and $\mathscr{B}_{2, \boldsymbol{\chi}}^{B}=r_{s}\left(\boldsymbol{\chi}, \mathbf{p}_{2}^{*}(\boldsymbol{\chi})\right)-\Lambda^{*} \mathbf{1}^{T} \mathbf{p}_{2}^{*}(\boldsymbol{\chi})$.

The physical interpretation of benefit function $\mathscr{B}_{k, \chi}^{B}$ represents the profit by using power policy $\mathbf{p}_{k}^{*}(\chi)$. The SBS will select the power strategy that returns the highest profit. Proof of Theorem 3.2.1 can be found in Appendix B.3.

\subsubsection{Optimal power policy for PTPC}

Note that by replacing the constraint (3.7b) with $\mathbf{1}^{T}\left(w(\boldsymbol{\chi}) \mathbf{p}_{1}(\boldsymbol{\chi})+(1-w(\boldsymbol{\chi})) \mathbf{p}_{2}(\boldsymbol{\chi})\right) \leq$ $P_{O}$, we will get the reformulated version of (P2). Following the same procedure as in the ATPC case, we start from KKT necessary and sufficient conditions provided in Appendix B.4. Providing that channel state is continuous, the optimal power policy can be summarized in Theorem 3.2.2.

Theorem 3.2.2. The optimal power control for problem (P2) is $\mathbf{P}^{*}=X_{w_{1}^{*}}(\chi) \mathbf{p}_{1}^{*}(\chi)+$ $\left(1-X_{w_{1}^{*}}(\chi)\right) \mathbf{p}_{2}^{*}(\chi)$, where $\Lambda^{*}(\chi)$ and $S^{*}$ are the solution to $\sum_{i=1}^{M} P_{i}^{*}(\chi) \leq P_{O}$ and $E\left[w^{*}(\chi)\right] \geq 1-\epsilon_{p}$ while $\mathbf{p}_{1}^{*}(\boldsymbol{\chi}), \mathbf{p}_{2}^{*}(\boldsymbol{\chi})$ and $w^{*}(\boldsymbol{\chi})$ are defined as follows

$$
\begin{aligned}
& p_{1 i}^{*}(\chi)= \begin{cases}P_{O} & , w^{*}(\chi)=1, P_{O} \leq p_{R P}^{*}(\chi), i=\arg \max _{m \in \mathcal{I}} z_{m} \\
p_{R P}^{*}(\chi) & , w^{*}(\boldsymbol{\chi})=1, P_{O}>p_{R P}^{*}(\chi), i=\arg \max _{m \in \mathcal{I}} z_{m} \\
0 & , \text { otherwise }\end{cases} \\
& p_{2 i}^{*}(\chi)= \begin{cases}P_{O} & , w^{*}(\chi)=0, i=\arg \max _{m \in \mathcal{I}} z_{m} \\
0 & , \text { otherwise }\end{cases} \\
& w^{*}(\chi)= \begin{cases}1, & \mathscr{B}_{1, \chi}^{B}>\mathscr{B}_{2, \chi}^{B} \\
0, & \mathscr{B}_{1, \chi}^{B}<\mathscr{B}_{2, \chi}^{B}\end{cases}
\end{aligned}
$$


where $\mathscr{B}_{1, \boldsymbol{\chi}}^{B}=r_{s}\left(\boldsymbol{\chi}, \mathbf{p}_{1}^{*}(\boldsymbol{\chi})\right)-\Lambda^{*}(\boldsymbol{\chi}) \mathbf{1}^{T} \mathbf{p}_{1}^{*}(\boldsymbol{\chi})+S^{*}$ and $\mathscr{B}_{2, \boldsymbol{\chi}}^{B}=r_{s}\left(\boldsymbol{\chi}, \mathbf{p}_{2}^{*}(\boldsymbol{\chi})\right)-\Lambda^{*}(\boldsymbol{\chi}) \mathbf{1}^{T} \mathbf{p}_{2}^{*}(\boldsymbol{\chi})$.

For The proof of Theorem 3.2.2, please refer to Appendix B.5.

Remark 3.2.1. $\mathscr{B}_{1, \chi}^{B}=\mathscr{B}_{2, \chi}^{B}$ happens with zero probability in continuous fading channel scenario.

\subsection{SU throughput scaling with ON-OFF power policy at $\mathrm{PU}$}

In this section, we investigate the SBS throughput (ergodic sum rate) scaling laws as the number of secondary users $M$ goes to infinity when the PU adopts an ONOFF power control policy. In order to do this, we make the additional assumption that all channel power gains are exponentially distributed with unity mean. We then analyze the asymptotic scaling properties of the ergodic sum rate according to the derived optimal power control laws for both ATPC and PTPC cases. From the derived optimal solution in the ATPC case, we can divide the channel state $\chi$ into four possible cases as shown in Table 3.2. Similarly, there are also four possible cases for the optimal power control solution derived for the PTPC case as shown in Table. 3.3 below.

Table 3.2: Four possible cases for the fading channel state $\chi$ with ATPC and ONOFF power policy at the $\mathrm{PU}$

\begin{tabular}{c|lcc}
\hline \hline Case & \multicolumn{1}{|c}{ Properties } & Power control & Outage at PU \\
\hline 1 & $g<g_{T}$ & $p_{W F, i^{*}}^{*}$ & Yes (PU turns OFF) \\
\hline 2 & $g \geq g_{T}, p_{W F, i^{*}}^{*} \leq p_{R P}^{*}$ & $p_{W F, i^{*}}^{*}$ & No \\
\hline 3 & $g \geq g_{T}, p_{W F, i^{*}}^{*} \geq p_{R P}^{*}, \mathscr{B}_{1, \chi}^{B} \leq \mathscr{B}_{2, \chi}^{B}$ & $p_{W F, i^{*}}^{*}$ & Yes \\
\hline 4 & $g \geq g_{T}, p_{W F, i^{*}}^{*} \geq p_{R P}^{*}, \mathscr{B}_{1, \chi}^{B}>\mathscr{B}_{2, \chi}^{B}$ & $p_{R P}^{*}$ & No \\
\hline \hline
\end{tabular}


Table 3.3: Four possible cases in fading channel state $\chi$ for PTPC and ON-OFF power policy at the $\mathrm{PU}$

\begin{tabular}{c|lcc}
\hline \hline Case & \multicolumn{1}{|c}{ Properties } & Power control & Outage at PU \\
\hline 1 & $g<g_{T}$ & $P_{O}$ & Yes (PU turn OFF) \\
\hline 2 & $g \geq g_{T}, P_{O} \leq p_{R P}^{*}$ & $P_{O}$ & No \\
\hline 3 & $g \geq g_{T}, P_{O} \geq p_{R P}^{*}, \mathscr{B}_{1, \chi}^{B} \leq \mathscr{B}_{2, \chi}^{B}$ & $P_{O}$ & Yes \\
\hline 4 & $g \geq g_{T}, P_{O} \geq p_{R P}^{*}, \mathscr{B}_{1, \chi}^{B}>\mathscr{B}_{2, \chi}^{B}$ & $p_{R P}^{*}$ & No \\
\hline \hline
\end{tabular}

Obviously, if $S^{*}=0$, it implies that SBS can transmit with $p_{W F}^{*}$ in ATPC case or $P_{O}$ in ATPC case without making POC active, as if the primary network never existed. This scenario then simplifies to a non-cognitive broadcast channel. With similar technique in $[54,58]$, we can show that the ergodic sum rate for a BC scales like $\log (\log M)$ in this case. Hence, this chapter will only study the case that $S^{*}>0$. Recall that $z_{i}=\frac{h_{i}}{\alpha_{i} P_{p}(g)+N_{0}}$. Let $F_{G}(g)$ be the CDF of $g$. First we find the CDF of $z_{i}$ when $g<g_{T}$ and $g \geq g_{T}$, denoted by $F_{Z}\left(z \mid \mathcal{S}_{1}\right)$ and $F_{Z}\left(z \mid \mathcal{S}_{1}^{c}\right)$, respectively. Here $\mathcal{S}_{1}$ denotes the set $g<g_{T}$ and $\mathcal{S}_{1}^{c}$ denotes the set $g \geq g_{T}$.

- When $g \geq g_{T}$, i.e. $\chi \in \mathcal{S}_{1}^{c}, z_{i}=\frac{h_{i}}{\alpha_{i} P_{c}+N_{0}}$ which is independent on the value of $g$. We can show that $F_{Z}\left(z \mid \mathcal{S}_{1}^{c}\right)=\left(1-\frac{\exp \left(-N_{0} z\right)}{1+P_{c} z}\right)$ and the probability density function $f_{Z}\left(z \mid \mathcal{S}_{1}^{c}\right)=\frac{d}{d z}\left[1-\frac{\exp \left(-N_{0} z\right)}{1+P_{c} z}\right]$. Since $\frac{1-F_{Z}\left(z \mid \mathcal{S}_{1}^{c}\right)}{f_{Z}\left(z \mid \mathcal{S}_{1}^{c}\right)}=\frac{P_{c} z+1}{N_{0} P_{c} z+N_{0}+P_{c}}$, $\lim _{z \rightarrow \infty} \frac{d}{d z}\left[\frac{1-F_{Z}\left(z \mid \mathcal{S}_{1}^{c}\right)}{f_{Z}\left(z \mid \mathcal{S}_{1}^{c}\right)}\right]=\lim _{z \rightarrow \infty}\left(\frac{P_{c}}{N_{0} P_{c} z+N_{0}+P_{c}}\right)^{2}=0$. It means that $F_{Z}\left(z \mid \mathcal{S}_{1}^{c}\right)$ belongs to the domain of attraction of the Gumbel distribution [82] as it satisfies the Von Mises conditions. When $g<g_{T}, z=\frac{h}{N_{0}}$ and it is now independent on $g$. Thus, the $\mathrm{CDF} z$ of is $F_{Z}\left(z \mid \mathcal{S}_{1}\right)=\left(1-\exp \left(-N_{0} z\right)\right)$. By the similar procedure, we can show that $F_{Z}\left(z \mid \mathcal{S}_{1}\right)$ also belongs to the domain of attraction of the Gumbel distribution.

- In order to analyze how the secondary throughput scales, we have to investigate the property of $z_{\max }=\max _{m \in \mathcal{I}} z_{m}$ for large $M$. The required result is provided in Lemma 3.3.1 as follows: 
Lemma 3.3.1. $\lim _{M \rightarrow \infty} \frac{E\left[\log \left(z_{\max }\right) 1_{\left\{z_{\max } \geq \Lambda^{*}\right\}} \mid \mathcal{S}_{1}^{c}\right]}{\log (\log M)}=1$ and $\lim _{M \rightarrow \infty} \frac{E\left[\log \left(z_{\max }\right) 1_{\left\{z_{\max } \geq \Lambda^{*}\right\}} \mid \mathcal{S}_{1}\right]}{\log (\log M)}=$ 1 .

Proof. Please refer to Appendix B.6.

- Define $\theta=\frac{\mathscr{P}_{p}(g)}{\beta}$. For $g \geq g_{T}, \theta=\frac{1}{\beta}\left(\frac{g P_{c}}{\exp \left(r_{p}^{0}\right)-1}-N_{0}\right)$. The CDF of $\theta$ given $g \geq g_{T}, F_{\theta}\left(\theta \mid \mathcal{S}_{1}^{c}\right)$, can be expressed as $1-\frac{1}{1+c_{o} \theta}$ where $c_{o}=\frac{\exp \left(r_{p}^{0}\right)-1}{P_{c}}=\frac{g_{T}}{N_{0}}$.

The above results will be used below to analyze the SBS throughput scaling laws as $M \rightarrow \infty$, for the ATPC case and the PTPC case, respectively.

\subsubsection{Throughput scaling in ATPC case}

From Table. 3.2, the channel states when $g \geq g_{T}$ can be illustrated by Figure. 3.2, all regions can be described as follows.

$$
\begin{aligned}
& \mathcal{S}_{1}=\left\{g<g_{T}\right\} \\
& \mathcal{S}_{2}=\left\{g \geq g_{T}, z_{\max } \leq \Lambda^{*}\right\} \bigcup\left\{g \geq g_{T}, z_{\max } \geq \Lambda^{*}, \frac{1}{\Lambda^{*}}-\frac{1}{z_{\max }} \leq \theta \leq \infty\right\} \\
& \mathcal{S}_{3}=\left\{g \geq g_{T}, z_{\max } \geq \frac{\Lambda^{*}}{k_{o}}, 0 \leq \theta \leq \frac{k_{o}}{\Lambda^{*}}-\frac{1}{z_{\max }}\right\} \\
& \mathcal{S}_{4}=\left\{g \geq g_{T}, z_{\max } \geq \Lambda^{*}, \frac{k_{o}}{\Lambda^{*}}-\frac{1}{z_{\max }} \leq \theta \leq \frac{1}{\Lambda^{*}}-\frac{1}{z_{\max }}\right\}
\end{aligned}
$$

where $k_{o}$ is the solution to $\log \left(k_{o}\right)-k_{o}+S^{*}+1=0$.

- Throughput $C_{s}^{*}=E\left[r_{s}\left(\boldsymbol{\chi}, \mathbf{P}^{*}(\boldsymbol{\chi})\right)\right]=E\left[r_{s}^{*}\right]$ can be computed as follows

$$
\begin{aligned}
C_{s}^{*}= & \sum_{k=1}^{4} \operatorname{Pr}\left(\mathcal{S}_{k}\right) E\left[r_{s}^{*} \mid \mathcal{S}_{k}\right] \\
= & \operatorname{Pr}\left(\mathcal{S}_{1}\right) E\left[\log \left(\frac{z_{\max }}{\Lambda^{*}}\right) 1_{\left\{z_{\max } \geq \Lambda^{*}\right\}} \mid \mathcal{S}_{1}\right]+\operatorname{Pr}\left(\mathcal{S}_{2}\right) E\left[\log \left(\frac{z_{\max }}{\Lambda^{*}}\right) 1_{\left\{z_{\max } \geq \Lambda^{*}\right\}} \mid \mathcal{S}_{2}\right] \\
& +\operatorname{Pr}\left(\mathcal{S}_{3}\right) E\left[\log \left(\frac{z_{\max }}{\Lambda^{*}}\right) 1_{\left\{z_{\max } \geq \Lambda^{*}\right\}} \mid \mathcal{S}_{3}\right]+\operatorname{Pr}\left(\mathcal{S}_{4}\right) E\left[\log \left(1+\theta z_{\max }\right) \mid \mathcal{S}_{4}\right]
\end{aligned}
$$

Hence, the upper-bound of $C_{s}^{*}$ is

$$
\begin{aligned}
C_{s}^{*} \leq & \operatorname{Pr}\left(\mathcal{S}_{1}\right) E\left[\log \left(\frac{z_{\max }}{\Lambda^{*}}\right) 1_{\left\{z_{\max } \geq \Lambda^{*}\right\}} \mid \mathcal{S}_{1}\right]+\operatorname{Pr}\left(\mathcal{S}_{2}\right) E\left[\log \left(\frac{z_{\max }}{\Lambda^{*}}\right) 1_{\left\{z_{\max } \geq \Lambda^{*}\right\}} \mid \mathcal{S}_{2}\right] \\
& +\operatorname{Pr}\left(\mathcal{S}_{3}\right) E\left[\log \left(\frac{z_{\max }}{\Lambda^{*}}\right) 1_{\left\{z_{\max } \geq \Lambda^{*}\right\}} \mid \mathcal{S}_{3}\right]+\operatorname{Pr}\left(\mathcal{S}_{4}\right) E\left[\log \left(\frac{z_{\max }}{\Lambda^{*}}\right) 1_{\left\{z_{\max } \geq \Lambda^{*}\right\}} \mid \mathcal{S}_{4}\right] \\
= & E\left[\log \left(\frac{z_{\max }}{\Lambda^{*}}\right) 1_{\left\{z_{\max } \geq \Lambda^{*}\right\}}\right]
\end{aligned}
$$




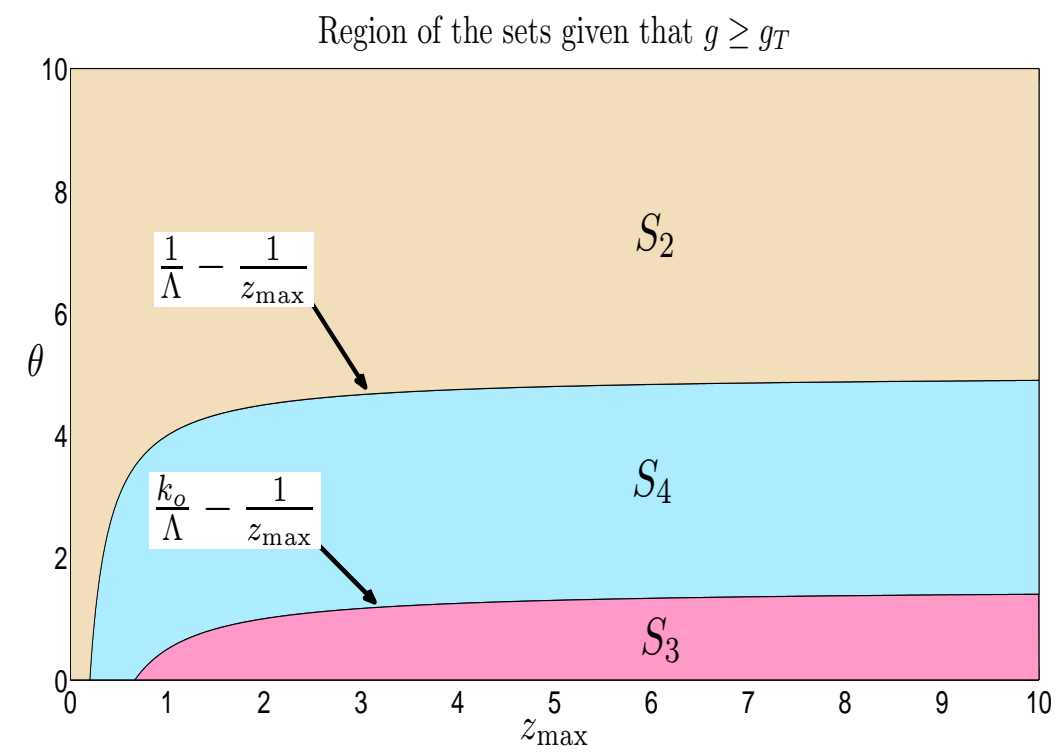

Figure 3.2: Region in ATPC case given that $g \geq g_{T}$ when the PU uses ON-OFF power strategy

- It is worth mentioning that $\Lambda^{*}$ varies with the number of SUs $M$, but we do not show it explicitly. The property of $\Lambda^{*}$ is emphasized in Lemma 3.3.2.

Lemma 3.3.2. $\Lambda^{*}$ has an upper-bound, i.e. $\Lambda^{*} \leq \frac{1}{P_{a v}}$, and it is bounded away from zero.

The proof of Lemma 3.3.2 can be found in Appendix B.7.

- Note that $k_{o}=1$ when $S^{*}=0, \operatorname{Pr}\left\{\mathcal{S}_{4}\right\}=0$, and $p_{R P}$ is not applied in any channel states. As explained earlier, the throughput scaling in this case has been analyzed already [54]. When $k_{o}$ approaches 0 , it implies that $\operatorname{Pr}\left\{\mathcal{S}_{3}\right\}=0$ and the total outage probability becomes $\operatorname{Pr}\left\{\mathcal{S}_{1}\right\}+\operatorname{Pr}\left\{\mathcal{S}_{3}\right\}=\epsilon_{p}^{0}+0=\epsilon_{p}^{0}$, implying that we set $\epsilon_{p}=\epsilon_{p}^{0}$. So, the analysis will be split into 2 parts. First is when $\epsilon_{p}>\epsilon_{p}^{0}\left(0<k_{o}<1\right)$ and the second is when $\epsilon_{p}=\epsilon_{p}^{0}\left(k_{o}=0\right)$. 


\section{Lower-bound on throughput when $\epsilon_{p}>\epsilon_{p}^{0}$}

In set $\mathcal{S}_{4}$, we know that $\frac{k_{o} z_{\max }}{\Lambda^{*}} \leq 1+\theta z_{\max } \leq \frac{z_{\max }}{\Lambda^{*}} . C_{s}^{*}$ is also lower-bounded by

$$
\begin{aligned}
C_{s}^{*} \geq & \operatorname{Pr}\left(\mathcal{S}_{1}\right) E\left[\log \left(\frac{z_{\max }}{\Lambda^{*}}\right) 1_{\left\{z_{\max } \geq \Lambda^{*}\right\}} \mid \mathcal{S}_{1}\right]+\operatorname{Pr}\left(\mathcal{S}_{2}\right) E\left[\log \left(\frac{z_{\max }}{\Lambda^{*}}\right) 1_{\left\{z_{\max } \geq \Lambda^{*}\right\}} \mid \mathcal{S}_{2}\right] \\
& +\operatorname{Pr}\left(\mathcal{S}_{3}\right) E\left[\log \left(\frac{z_{\max }}{\Lambda^{*}}\right) \mid \mathcal{S}_{3}\right]+\operatorname{Pr}\left(\mathcal{S}_{4}\right) E\left[\log \left(\frac{k_{o} z_{\max }}{\Lambda^{*}}\right) \mid \mathcal{S}_{4}\right] \\
= & E\left[\log \left(\frac{z_{\max }}{\Lambda^{*}}\right) 1_{\left\{z_{\max } \geq \Lambda^{*}\right\}}\right]+\operatorname{Pr}\left\{\mathcal{S}_{4}\right\} \log \left(k_{o}\right) \\
\geq & E\left[\log \left(\frac{z_{\max }}{\Lambda^{*}}\right) 1_{\left\{z_{\max } \geq \Lambda^{*}\right\}}\right]+\log \left(\frac{\Lambda^{*}}{c_{o}} \cdot \frac{\gamma_{\epsilon}}{1-\gamma_{\epsilon}}\right)
\end{aligned}
$$

where the last inequality is from the lower bound of $k_{o}$ and $\gamma_{\epsilon}=\frac{\epsilon_{p}-\epsilon_{p}^{0}}{1-\epsilon_{p}^{0}}>0$, whose corresponding proof in Appendix B.8 shows that $\frac{\Lambda^{*}}{c_{o}}\left(\frac{\gamma_{\epsilon}}{1-\gamma_{\epsilon}}\right) \leq k_{o}<1$.

\section{Lower-bound on throughput when $\epsilon_{p}=\epsilon_{p}^{0}$}

In this case, the region $\mathcal{S}_{3}$ disappears. Therefore, the total capacity $C_{s}^{*}$ is

$$
\operatorname{Pr}\left\{g<g_{T}\right\} E\left[r_{s}^{*} \mid g<g_{T}\right]+\operatorname{Pr}\left\{g \geq g_{T}\right\} E\left[r_{s}^{*} \mid g \geq g_{T}\right] .
$$

In Appendix B.9, we have shown that

- If $\Lambda^{*} \geq c_{o}$, then $E\left[r_{s}^{*} \mid g \geq g_{T}\right] \geq E\left[\log \left(\frac{z_{\max }}{\Lambda^{*}}\right) 1_{\left\{z_{\max } \geq \Lambda^{*}\right\}} \mid g \geq g_{T}\right]+\frac{\frac{c_{o}}{\Lambda^{*}}}{1-\frac{c_{o}}{\Lambda^{*}}} \log \frac{c_{o}}{\Lambda^{*}}$

- If $\Lambda^{*} \leq c_{o}$, then $E\left[r_{s}^{*} \mid g \geq g_{T}\right] \geq E\left[\log \left(\frac{z_{\max }}{c_{o}}\right) 1_{\left\{z_{\max } \geq c_{o}\right\}} \mid g \geq g_{T}\right]+\frac{1}{1-\frac{c_{o}}{\Lambda^{*}}} \log \frac{c_{o}}{\Lambda^{*}}$

Theorem 3.3.1. When PU uses ON-OFF with constant power control, the behavior of throughput from the SBS under a POC and an ATPC with optimal power control can be described as follows.

$$
\lim _{M \rightarrow \infty} \frac{C_{s}^{*}}{\log (\log M)}=1
$$

The proof of Theorem 3.3.1 are summarized in Appendix B.10, through the results from Appendix B.6 to B.9.

\subsubsection{Throughput scaling in PTPC case}

In order to analyze the asymptotic scaling laws of the SBS ergodic sum rate, we first observe the following facts from Table. 3.3. 
- Given $g \geq g_{T}, P_{o} \leq \theta<\infty$ in case 2. For $0 \leq \theta \leq P_{o}$, we have case 3 and 4 which are separated by the curved $K\left(z_{\max }\right)$. The function $K\left(z_{\max }\right)$ is derived from the condition that $\mathscr{B}_{1, \chi}^{B}=\mathscr{B}_{2, \chi}^{B}$, whose expression and property are summarized in Lemma 3.3.3.

Lemma 3.3.3. The properties of $K\left(z_{\max }\right)$ are as follows:

$$
\begin{aligned}
& -K\left(z_{\max }\right)=-\frac{1}{z_{\max }}+\left(\frac{1}{z_{\max }}+P_{o}\right) \exp \left(-S^{*}-\frac{z_{\max }}{1+P_{o} z_{\max }}\right) . \\
& - \text { For } S^{*}>0, K^{\prime}\left(z_{\max }\right)>0 \text {, i.e. } K\left(z_{\max }\right) \text { is a strictly increasing function } \\
& \quad \text { if POC is active. }
\end{aligned}
$$

The proof of are provided in Appendix B.11. Let $z_{o}$ be the unique root of the equation $K\left(z_{\max }\right)=0$. (As we consider the case that POC is active, $S^{*}>0$ and the root is unique as $K\left(z_{\max }\right)$ is a strictly increasing function.) The regions when $g \geq g_{T}$ are illustrated by Figure. 3.3. Finally, we can identify the regions as follows.

$$
\begin{aligned}
\mathcal{S}_{1} & =\left\{g<g_{T}\right\} \\
\mathcal{S}_{2}= & \left\{g \geq g_{T}, 0 \leq z_{\max }<\infty, P_{o} \leq \theta \leq \infty\right\} \\
\mathcal{S}_{3}= & \left\{g \geq g_{T}, z_{o} \leq z_{\max }<\infty, 0 \leq \theta \leq K\left(z_{\max }\right)\right\} \\
\mathcal{S}_{4}= & \left\{g \geq g_{T}, z_{o} \leq z_{\max }<\infty, K\left(z_{\max }\right) \leq \theta \leq P_{o}\right\} \\
& \bigcup\left\{g \geq g_{T}, 0 \leq z_{\max }<z_{o}, 0 \leq \theta \leq P_{o}\right\}
\end{aligned}
$$

- Notice that, as $S^{*}$ approaches $\infty, \lim _{S^{*} \rightarrow \infty} K\left(z_{\max }\right)=-\frac{1}{z_{\max }}<0$ which means that $\lim _{S^{*} \rightarrow \infty} z_{o}$ tends to infinity. Thus, the region $\mathcal{S}_{3}$ vanishes, implying that $\epsilon_{p}^{0}=\operatorname{Pr}\left\{\mathcal{S}_{1}\right\}=\epsilon_{p}$. Hence, the analysis on the lower bound of throughput is split into two parts, i.e. when $\epsilon_{p}>\epsilon_{p}^{0}\left(0<S^{*}<\infty\right)$ and when $\epsilon_{p}=\epsilon_{p}^{0}$ $\left(S^{*}=\infty\right)$. 


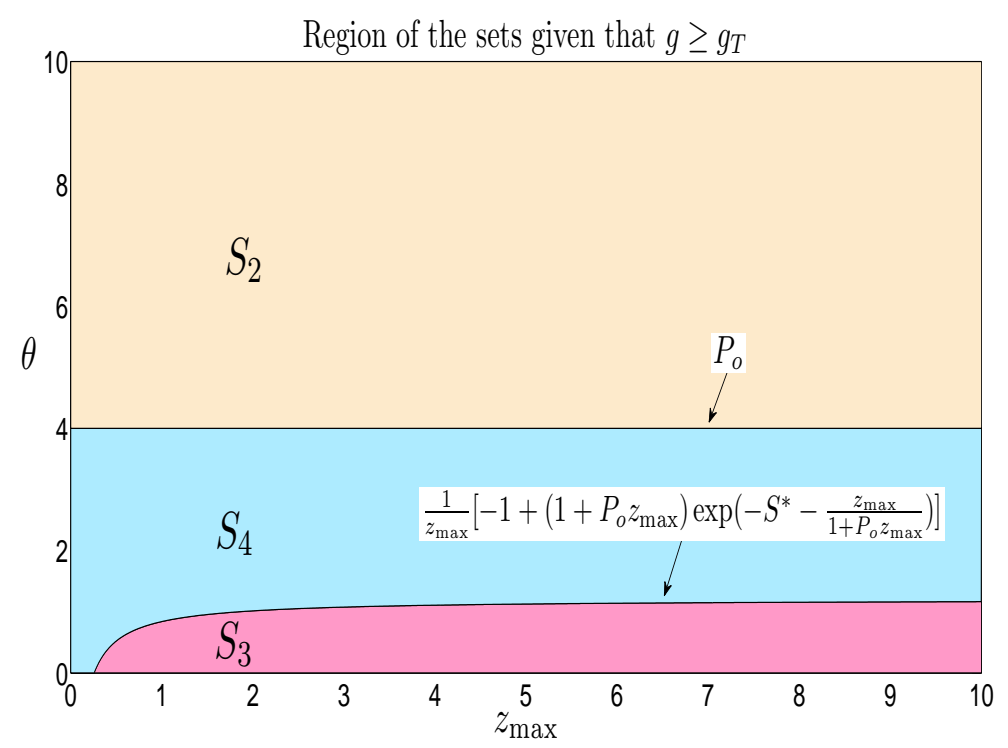

Figure 3.3: Region in PTPC case given that $g \geq g_{T}$ when the PU uses ON-OFF power strategy

- Throughput $C_{s}^{*}=E\left[r_{s}^{*}\right]$ can be calculated as follows

$$
\begin{aligned}
C_{s}^{*}= & \sum_{k=1}^{4} \operatorname{Pr}\left(\mathcal{S}_{k}\right) E\left[r_{s}^{*} \mid \mathcal{S}_{k}\right] \\
= & \operatorname{Pr}\left(\mathcal{S}_{1}\right) E\left[\log \left(1+P_{o} z_{\max }\right) \mid \mathcal{S}_{1}\right]+\operatorname{Pr}\left(\mathcal{S}_{2}\right) E\left[\log \left(\log \left(1+P_{o} z_{\max }\right) \mid \mathcal{S}_{2}\right]\right. \\
& +\operatorname{Pr}\left(\mathcal{S}_{3}\right) E\left[\log \left(1+P_{o} z_{\max }\right) \mid \mathcal{S}_{3}\right]+\operatorname{Pr}\left(\mathcal{S}_{4}\right) E\left[\log \left(1+\theta z_{\max }\right) \mid \mathcal{S}_{4}\right]
\end{aligned}
$$

Hence, the upper-bound of $C_{s}^{*}$ is

$$
\begin{aligned}
C_{s}^{*} \leq & \operatorname{Pr}\left(\mathcal{S}_{1}\right) E\left[\log \left(1+P_{o} z_{\max }\right) \mid \mathcal{S}_{1}\right]+\operatorname{Pr}\left(\mathcal{S}_{2}\right) E\left[\log \left(\log \left(1+P_{o} z_{\max }\right) \mid \mathcal{S}_{2}\right]\right. \\
& +\operatorname{Pr}\left(\mathcal{S}_{3}\right) E\left[\log \left(1+P_{o} z_{\max }\right) \mid \mathcal{S}_{3}\right]+\operatorname{Pr}\left(\mathcal{S}_{4}\right) E\left[\log \left(1+P_{o} z_{\max }\right) \mid \mathcal{S}_{4}\right] \\
= & E\left[\log \left(1+P_{o} z_{\max }\right)\right]
\end{aligned}
$$

\section{Lower-bound on throughput when $\epsilon_{p}>\epsilon_{p}^{0}$}

$$
\begin{aligned}
C_{s}^{*} & =\sum_{k=1}^{4} \operatorname{Pr}\left(\mathcal{S}_{k}\right) E\left[r_{s}^{*} \mid \mathcal{S}_{k}\right] \\
& \geq \sum_{k=1}^{4} \operatorname{Pr}\left(\mathcal{S}_{k}\right) E\left[\log \left(1+P_{o} z_{\max }\right) \mid \mathcal{S}_{k}\right]-\operatorname{Pr}\left\{\mathcal{S}_{4}\right\} \delta\left(P_{o}, c_{o}, \gamma_{\epsilon}\right) \\
& \geq E\left[\log \left(1+P_{o} z_{\max }\right)\right]-\delta\left(P_{o}, c_{o}, \gamma_{\epsilon}\right)
\end{aligned}
$$


where $\delta\left(P_{o}, c_{o}, \gamma_{\epsilon}\right)=\log \left(P_{o} c_{o} \frac{\gamma_{\epsilon}}{1-\gamma_{\epsilon}}\right)$ and $\gamma_{\epsilon}=\frac{\epsilon_{p}-\epsilon_{p}^{0}}{1-\epsilon_{p}^{0}}$. Appendix B.12 provides the details of proof in this case.

\section{Lower-bound on throughput when $\epsilon_{p}=\epsilon_{p}^{0}$}

In this case, the region $\mathcal{S}_{3}$ vanishes. Thus, SU sum downlink capacity becomes $\operatorname{Pr}\left\{g<g_{T}\right\} E\left[r_{s}^{*} \mid g<g_{T}\right]+\operatorname{Pr}\left\{g \geq g_{T}\right\} E\left[r_{s}^{*} \mid g \geq g_{T}\right]$, where $E\left[r_{s}^{*} \mid g<g_{T}\right]=$ $E\left[\log \left(1+P_{o} z_{\max }\right) \mid g<g_{T}\right]$ and it is shown in Appendix B.13 that $E\left[r_{s}^{*} \mid g \geq g_{T}\right] \geq$ $E\left[\log \left(\frac{z_{\max }}{c_{o}} 1\left(z \geq c_{o}\right)\right) \mid g \geq g_{T}\right]-\log \left(\frac{P_{o}+\frac{1}{c_{o}}}{P_{o}}\right)$.

Theorem 3.3.2. When $P U$ uses ON-OFF with constant power control, the behavior of throughput from the SBS under a POC and a PTPC with optimal power control can be described as follows.

$$
\lim _{M \rightarrow \infty} \frac{C_{s}^{*}}{\log (\log M)}=1
$$

The proof of Theorem 3.3.2 are summarized in Appendix B.14, through the results in Appendix B.6 and Appendix B.11 to B.13.

\subsection{SU throughput scaling with TCI power policy at PU}

In this section we focus on the case where the PU uses the TCI policy and derive asymptotic scaling laws of the SBS ergodic sum capacity when the number of SUs, $M \rightarrow \infty$, under the assumption (identical to Section 3.3) that all channel power gains are independent and identically exponentially distributed with unity mean. Again, we first divide the channel state $\boldsymbol{\chi}$ into specific sets with the aid of the optimal power allocation solutions in Theorem 3.2.1 for ATPC and in Theorem 3.2.2 for PTPC, respectively shown in Table 3.4 and Table 3.5.

Under the truncated channel inversion policy, PU is ON with $P_{p}(g)=\frac{k_{1}}{g}$ where $k_{1}=\left(e^{r_{p}^{0}}-1\right) N_{0}$. In this scenario, no $\mathrm{SU}$ is allowed to transmit if $\mathscr{B}_{1, \boldsymbol{\chi}}^{B}>\mathscr{B}_{2, \boldsymbol{\chi}}^{B}$, since $p_{R P}^{*}(\chi)=0$. Similar to the previous section, we will analyze the throughput 
Table 3.4: Three possible cases in fading channel state $\boldsymbol{\chi}$ for ATPC with TCI power policy at the PU

\begin{tabular}{c|cccc}
\hline \hline Case & Properties & Power control & Outage at PU & Probability \\
\hline 1 & $g<g_{T}$ & $p_{W F}^{*}$ & Yes (PU turn OFF) & $\epsilon_{p}^{0}$ \\
\hline 2 & $g \geq g_{T}, \mathscr{B}_{1, \chi}^{B} \leq \mathscr{B}_{2, \chi}^{B}$ & $p_{W F}^{*}$ & Yes & $\epsilon_{p}-\epsilon_{p}^{0}$ \\
\hline 3 & $g \geq g_{T}, \mathscr{B}_{1, \chi}^{B}>\mathscr{B}_{2, \chi}^{B}$ & $p_{R P}^{*}=0$ & No & $1-\epsilon_{p}$ \\
\hline \hline
\end{tabular}

Table 3.5: Three possible cases in fading channel state $\boldsymbol{\chi}$ for PTPC with TCI power policy at the PU

\begin{tabular}{c|cccc}
\hline \hline Case & Properties & Power control & Outage at PU & Probability \\
\hline 1 & $g<g_{T}$ & $P_{O}$ & Yes $($ PU turn OFF $)$ & $\epsilon_{p}^{0}$ \\
\hline 2 & $g \geq g_{T}, \mathscr{B}_{1, \chi}^{B} \leq \mathscr{B}_{2, \chi}^{B}$ & $P_{O}$ & Yes & $\epsilon_{p}-\epsilon_{p}^{0}$ \\
\hline 3 & $g \geq g_{T}, \mathscr{B}_{1, \chi}^{B}>\mathscr{B}_{2, \chi}^{B}$ & $p_{R P}^{*}=0$ & No & $1-\epsilon_{p}$ \\
\hline \hline
\end{tabular}

scaling laws only for the case when $S^{*}>0$. Define $\rho_{i}=\frac{h_{i}}{\alpha_{i} P_{p}(g)+N_{0}}$. The optimal SBS power allocation solution dictates that the SBS will transmit to the user with the maximum $\rho$, i.e. $\rho_{\max }$, for both ATPC and PTPC. It is also worth mentioning that we know the exact probability for each case of the channel fading state, as shown in Tables 3.4 and 3.5, so a complicated throughput analysis by using conditional CDFs as used in Section 3.3, can be avoided.

First, note that $\max \frac{h_{i}}{\frac{k_{1}}{g_{T}} \alpha_{i}+N_{0}} \leq \rho_{\max } \leq \max \frac{h_{i}}{N_{0}}$ regardless the value of $g$. Existing results from $[58,83]$ can be used to show that both $\max \frac{h_{i}}{\frac{k_{1}}{g_{T}} \alpha_{i}+N_{0}}$ and max $\frac{h_{i}}{N_{0}}$ scale like $\log M$ as $M \rightarrow \infty$. Thus, as $M$ grows large, $\rho_{\max }$ also scales like $\log M$. Next, we provide our detailed analysis of the SBS ergodic sum capacity scaling laws for ATPC in Section 3.4.1 and PTPC in Section 3.4.2. 


\subsubsection{Throughput scaling in ATPC case}

Assume that $S^{*}>0$. From Table. 3.4, we can seperate the region 2 and 3 by setting $\mathscr{B}_{1, \chi}^{B}=\mathscr{B}_{2, \chi}^{B}$, where $\mathscr{B}_{1, \chi}^{B}=S^{*}, \mathscr{B}_{2, \chi}^{B}=\log \left(1+\rho_{\max } p_{W F}^{*}\right)-\Lambda^{*} p_{W F}^{*}$, and $p_{W F}^{*}=\left(\frac{1}{\Lambda^{*}}-\frac{1}{\rho_{\max }}\right)^{+}$. Thus, it yields

$$
S^{*}=\log \left(\frac{\rho_{\max }}{\Lambda^{*}}\right)-\left(1-\frac{\Lambda^{*}}{\rho_{\max }}\right)=-\log (\Omega)+\Omega-1=t(\Omega)
$$

where $\Omega=\frac{\Lambda^{*}}{\rho_{\max }}$ and $0 \leq \Omega \leq 1$. Further note that $t(\Omega)$ is a decreasing function in $\Omega$. Thus, the region can also be expressed as follows

$$
\begin{aligned}
& \tilde{\mathcal{S}}_{1}=\left\{g<g_{T}\right\} \\
& \tilde{\mathcal{S}}_{2}=\left\{g \geq g_{T}, 0 \leq \Omega \leq t^{-1}\left(S^{*}\right)\right\} \quad=\left\{g \geq g_{T}, \frac{\Lambda^{*}}{t^{-1}\left(S^{*}\right)} \leq \rho_{\max }<\infty\right\} \\
& \tilde{\mathcal{S}}_{3}=\left\{g \geq g_{T}, 0 \leq \rho_{\max } \leq \frac{\Lambda^{*}}{t^{-1}\left(S^{*}\right)}\right\}
\end{aligned}
$$

where $t^{-1}($.$) is inverse function of t$.

With the similar procedure as in Appendix B.7 in ATPC case for ON-OFF power control at the PU, we can show that $\Lambda^{*}$ will not converge to zero for all $M$. Further, as the probability of $\tilde{\mathcal{S}}_{2}$ and $\tilde{\mathcal{S}}_{3}$ are fixed, we can come up with a property of $\frac{1}{t^{-1}\left(S^{*}\right)}$ as shown in Lemma 3.4.1.

Lemma 3.4.1. For $\epsilon_{p}>\epsilon_{p}^{0}, \frac{1}{t^{-1}\left(S^{*}\right)}=\Theta(\log M)$.

Proof. As $\rho_{\max }$ scales like $\log M$, we first assume that $\frac{1}{t^{-1}\left(S^{*}\right)}$ grows faster than $\log M$. It implies that, for $M$ large enough, $\operatorname{Pr}\left(\tilde{\mathcal{S}}_{2}\right)$ converges to 0 , which contradicts the fact that $\operatorname{Pr}\left(\tilde{\mathcal{S}}_{2}\right)=\epsilon_{p}-\epsilon_{p}^{0}>0$ for all $M$. Next, assume that $\frac{1}{t^{-1}\left(S^{*}\right)}$ grows slower than $\log M$. It means that, when $M$ is large enough, $\operatorname{Pr}\left(\tilde{\mathcal{S}}_{3}\right)$ converges to 0 , which contradicts the fact that $\operatorname{Pr}\left(\tilde{\mathcal{S}}_{3}\right)=1-\epsilon_{p}>0$ for all $M$. Thus, $\frac{1}{t^{-1}\left(S^{*}\right)}=$ $\Theta(\log M)$.

Theorem 3.4.1. When PU uses TCI power control, the behavior of throughput from the SBS under a POC and an ATPC with optimal power control can be described as follows.

$$
\lim _{M \rightarrow \infty} \frac{C_{s}^{*}}{\log (\log M)}=\epsilon_{p}
$$


For the case $\epsilon_{p}=\epsilon_{p}^{0}$, the proof is in Appendix B.15. The result from Lemma 3.4.1 is useful to prove Theorem 3.4.1 when $\epsilon_{p}>\epsilon_{p}^{0}$ whose details are provided in Appendix B.16.

The intuitive explanation for Theorem 3.4.1 is that when PU uses TCI power control, the SBS are forced to turn off if it decides to protect primary link's QoS because even small amount of power can put PU in an outage. As the primary outage probability threshold is $\epsilon_{p}$, it means SBS are allowed to transmit with probability $\epsilon_{p}$ which leads to the pre-log factor term.

\subsubsection{Throughput scaling in PTPC case}

Assume that $S^{*}>0$. From Table. 3.4, we can seperate the region 2 and 3 by setting $\mathscr{B}_{1, \chi}^{B}=\mathscr{B}_{2, \boldsymbol{\chi}}^{B}$, where $\mathscr{B}_{1, \boldsymbol{\chi}}^{B}=S^{*}$ and $\mathscr{B}_{2, \boldsymbol{\chi}}^{B}=\log \left(1+\rho_{\max } P_{O}\right)-\Lambda^{*}(\boldsymbol{\chi}) P_{O}$. Note that in PTPC case, $\Lambda^{*}(\chi)=\frac{\rho_{\max }}{1+\rho_{\max } P_{O}}$ if $P_{O}$ is applied. Thus, it yields

$$
S^{*}=\log \left(1+\rho_{\max } P_{O}\right)-\frac{\rho_{\max } P_{O}}{1+\rho_{\max } P_{O}}=-\log (\omega)+\omega-1=t(\omega)
$$

where $\omega=\frac{1}{1+\rho_{\max } P_{O}}$ and $0 \leq \omega \leq 1$. Further note that $t(\omega)$ is a decreasing function in $\omega$. Thus, the region can also be expressed as follows

$$
\begin{aligned}
& \tilde{\mathcal{S}}_{1}=\left\{g<g_{T}\right\} \\
& \tilde{\mathcal{S}}_{2}=\left\{g \geq g_{T}, 0 \leq \omega \leq t^{-1}\left(S^{*}\right)\right\} \quad=\left\{g \geq g_{T}, \frac{1}{P_{O}}\left(\frac{1}{t^{-1}\left(S^{*}\right)}-1\right) \leq \rho_{\max }<\infty\right\} \\
& \tilde{\mathcal{S}}_{3}=\left\{g \geq g_{T}, 0 \leq \rho_{\max } \leq \frac{1}{P_{O}}\left(\frac{1}{t^{-1}\left(S^{*}\right)}-1\right)\right\}
\end{aligned}
$$

where $t^{-1}($.$) is inverse function of t$.

Similar to the proof of Lemma 3.4.1 in ATPC case, we can show that, for $\epsilon_{p}>\epsilon_{p}^{0}$, $\frac{1}{t^{-1}\left(S^{*}\right)}=\Theta(\log M)$. The result will help use prove the result in Theorem 3.4.2 when $\epsilon_{p}>\epsilon_{p}^{0}$, which is in Appendix B.18. For $\epsilon_{p}=\epsilon_{p}^{0}$, the details of the proof is in Appendix B.17.

Theorem 3.4.2. When PU uses TCI power control, the behavior of throughput from the SBS under a POC and a PTPC with optimal power control can be described as 
follows.

$$
\lim _{M \rightarrow \infty} \frac{C_{s}^{*}}{\log (\log M)}=\epsilon_{p}
$$

The intuitive reason for the appearence of the pre-log factor $\epsilon_{p}$ is similar to ATPC case with TCI power control at PU.

\subsection{Numerical results}

In this section, we present some numerical results on the performance of the proposed optimal power policies for the capacity maximization problem with POC and ATPC or PTPC. All channel gains involved are assumed to be Rayleigh fading and the corresponding channel power gains are taken to be exponentially distributed with unit mean. Noises at PR and all SRs are presumed to be equal and AWGN with unit variance, i.e. $N_{0}=1$. Note that this allows the transmit power at the primary and secondary transmitters to be interpreted as signal-to-noise ratio at the transmitter side. Unless specified otherwise, the constant power $P_{c}$ for the primary ON-OFF power policy is set to be $15 \mathrm{~dB}$ when it is ON. PU's target rate is $r_{p}^{0}=1.25$ nats per channel use and the primary outage probability threshold $\epsilon_{p}=0.1$. For the primary TCI power policy, we set $\epsilon_{p}^{0}=0.05$. For convenience, we further assume that all secondary receivers are identical. The simulation results are based on a Monte-Carlo method averaged over $10^{5}$ channel realizations.

\subsubsection{The effect of POC on sum ergodic capacity in C-BC channel}

Figures 3.4 and 3.5 exhibit the effect of the average transmit power budget on the SBS downlink sum capacity when PU uses an ON-OFF power policy with a constant power when ON for both with and without POC. Fig.3.4 shows that with an increasing average power budget $\left(P_{a v}\right)$, SBS downlink sum capacity increases for both with and without POC when PU applies the ON-OFF power strategy, as expected. The same feature is also noticed for the PTPC case, shown in Fig.3.5. However, the POC becomes the dominant constraint when the average power budget 
is raised further, making the rate of increase in the SBS downlink sum capacity drop significantly compared to the case without POC. The implication of the optimal power policies with POC is that when POC becomes active, SBS is forced to transmit with the highest possible power that still guarantees PU's service rate, i.e. $p_{R P}^{*}$, for some channel realizations. Nevertheless, PU can still face outage either due to its own transmission strategy (when PU is OFF) or when SBS chooses to transmit to maximize its sum capacity even though this strategy causes an outage to the PU. It is also worth pointing out that Figures 3.4 and 3.5 show the benefit of multiuser diversity. As the number of SRs $M$ increases, the SBS downlink throughput is enhanced as SBS has a statistically higher opportunity to obtain a high value of $z_{\max }$. Detailed throughput scaling results are discussed next.

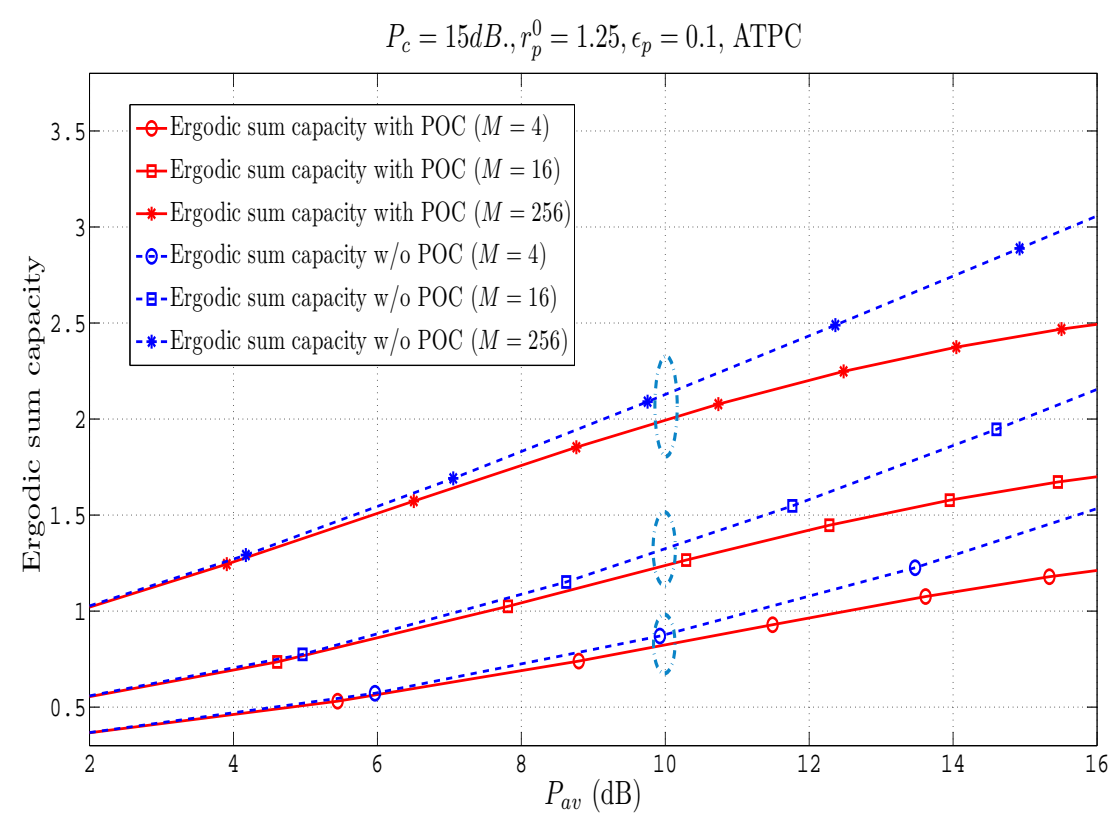

Figure 3.4: SU ergodic sum capacity in BC problem against average transmit power budget : $r_{p}^{0}=1.25, \epsilon_{p}=0.1$, and $P_{c}=15 \mathrm{~dB}$. with $\mathrm{ON}-\mathrm{OFF}$ power policy at the $\mathrm{PU}$

\subsubsection{Throughput scaling results in the secondary network}

This part presents the numerical results which affirm the throughput scaling results of Theorems 3.3.1 to 3.4.2. We also present some numerical results and intuitive explanations regarding how the individual parameters of the problem setup affect the SBS downlink throughput. 


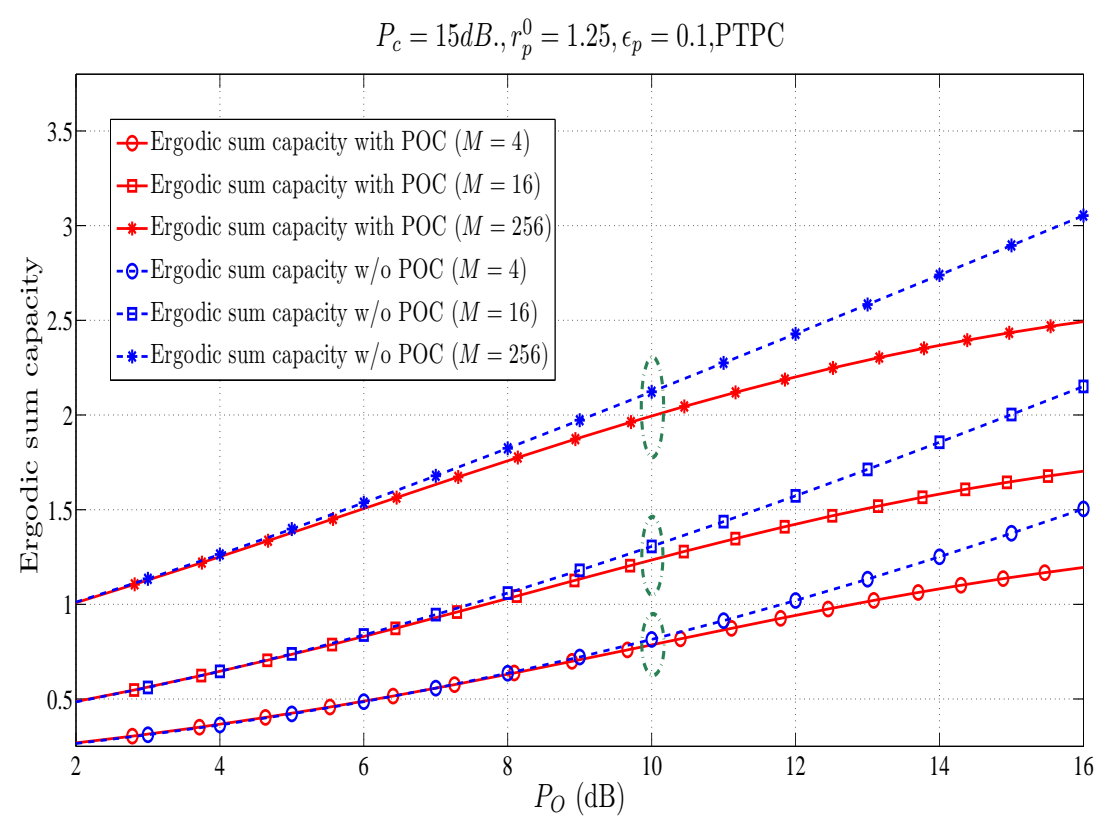

Figure 3.5: SU ergodic sum capacity in $\mathrm{BC}$ problem against peak transmit power budget : $r_{p}^{0}=1.25, \epsilon_{p}=0.1$, and $P_{c}=15 \mathrm{~dB}$. with $\mathrm{ON}-\mathrm{OFF}$ power policy at the $\mathrm{PU}$

\section{Throughput scaling results with ON-OFF power policy at the PU}

Fig.3.6 depicts the normalized throughput $\frac{C_{s}}{\log (\log M)}$ as a function of secondary receivers $M$ for both ATPC and PTPC when the PU utilizes the ON-OFF power policy. For these simulations, $P_{a v}$ for the ATPC case and $P_{O}$ for the PTPC case are set to $8 \mathrm{~dB}$. The results reveal that the normalized throughput converges to 1 in both cases, as stated in Theorems 3.3.1 and 3.3.2. Note that the convergence is asymptotic in nature and for a finite number of SUs, the gap between the normalized throughput and its asymptotic value can be appreciable.

The number of parameters affecting the SBS sum throughput include the constant power $P_{c}$ at the PU, the PU's target rate $r_{p}^{0}$, the PU's outage probability constraint $\epsilon_{p}$ and the power budget at SBS ( $P_{a v}$ for ATPC and $P_{O}$ for PTPC). In Fig.3.7, we illustrate the effect of these parameters on the SBS sum throughput with ATPC. We use the parameter values $\left(r_{p}^{0}=1.25, P_{c}=15 d B ., P_{a v}=15 d B ., \epsilon_{p}=0.1\right)$ in Fig.3.7 as the reference for the ATPC case. For convenience, the graph $\log \log M$ is also plotted as a reference.

First, we look at the effect of secondary power budget on the SBS sum throughput. A decrease in the secondary power budget significantly reduces the SU sum 
throughput as expected. Next, if $P_{c}$ is raised while the other variables are kept fixed, $g_{T}=\frac{\left(e^{r_{p}^{0}}-1\right) N_{0}}{P_{c}}$ is reduced, making $\epsilon_{p}^{0}$ decrease, and the additional outage, $\epsilon_{p}-\epsilon_{p}^{0}$, caused by the SBS increase. Thus, SBS sum throughput increases as a result. Another variable that affects $g_{T}$ is $r_{p}^{0}$ since $g_{T}$ decreases if $r_{p}^{0}$ is decreased, leading to the an increase in the sum throughput. Intuitively, it means that POC is less strict as $r_{p}^{0}$ is reduced. Finally, the PU outage probability threshold $\epsilon_{p}$ affects the throughput scaling as it also directly affects the POC. From Fig.3.7, it is seen that the SBS sum throughput shifts downwards as $\epsilon_{p}$ is reduced to 0.08 . This is obviously due to the fact that the POC becomes stricter. It is observable that the results with PTPC in Fig.3.8 are quite similar to ATPC case and the corresponding explanation are excluded to avoid repetition. Note also that the gap between the sum throughput curves and the first order approximation represented by the $\log \log M$ curve can be attributed to the second order approximations which are not explicitly shown here. For more details on the second order approximations and related explanations on the cognitive multiple-access channel case, see [58].

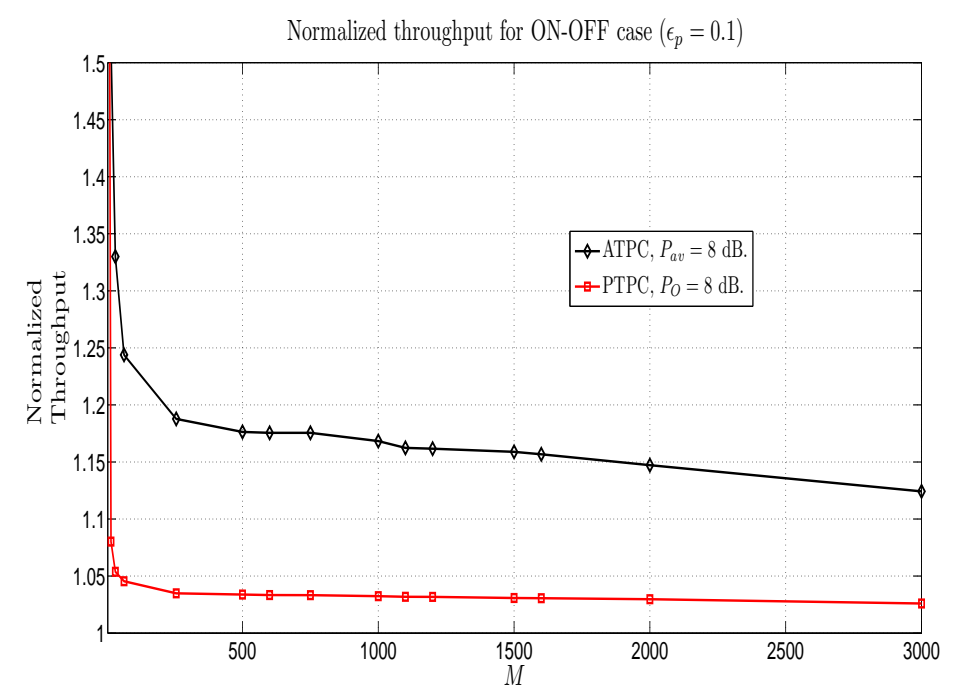

Figure 3.6: Normalized SBS sum throughput with ON-OFF power policy at the PU

\section{Throughput scaling results with TCI power policy at the PU}

In this part, the TCI power policy is assumed at the PU. We plot the normalized throughput $\frac{C_{s}}{\log (\log M)}$ as a function of secondary receivers $M$ for both ATPC and PTPC in in order to illustrate the results of Theorem 5 and $6 . P_{a v}$ in ATPC case 


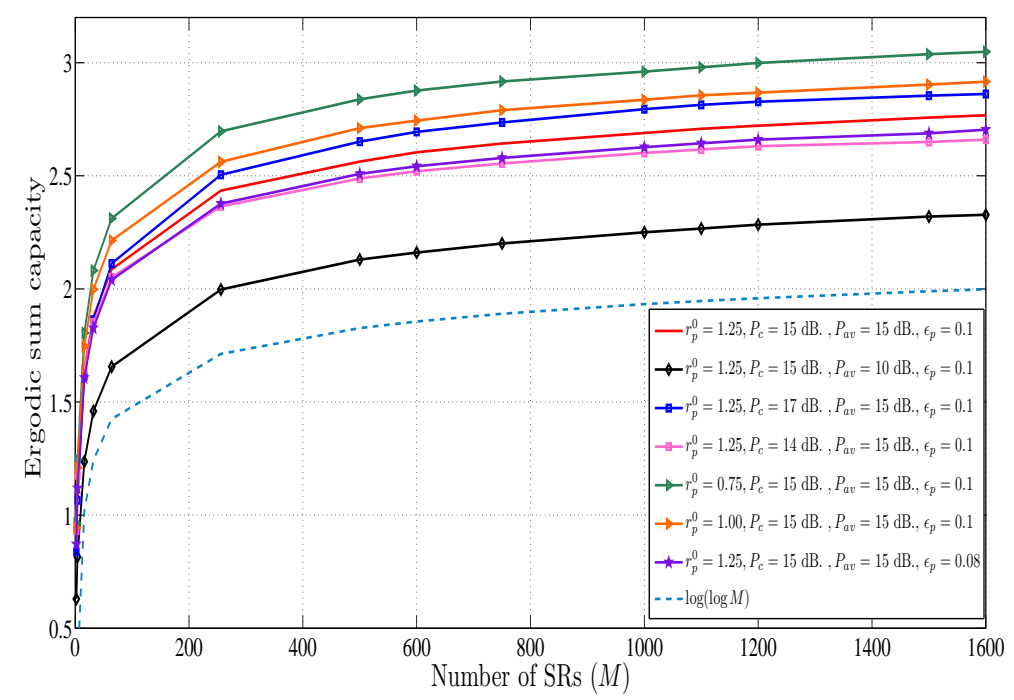

Figure 3.7: The effect of related parameters on SBS sum throughput with POC against $M$ for ON-OFF power policy at PU and ATPC

and $P_{O}$ in PTPC case are set to be $1 \mathrm{~dB}$. The results in Fig.3.9 shows that the normalized throughput converges to $\epsilon_{p}$ as $M$ is large enough. Intuitively, it is because the secondary network cannot transmit within $1-\epsilon_{p}$ fraction of time.

In the scenario with TCI case, there are also various variables which affect the SU throughput scaling, including the value of $\epsilon_{p}^{0}$, the PU's target rate $r_{p}^{0}$, PU outage probability constraint $\epsilon_{p}$ and the secondary network's power budget ( $P_{a v}$ for ATPC and $P_{O}$ for PTPC). Fig.3.10 and Fig.3.11 depict the effect of those variables on the $\mathrm{SU}$ throughput scaling. We set the case $\left(r_{p}^{0}=1.25, \epsilon_{p}^{0}=0.05, P_{a v}=5 d B ., \epsilon_{p}=0.1\right)$ in Fig.3.10 as the reference of ATPC case and the case $\left(r_{p}^{0}=1.25, \epsilon_{p}^{0}=0.05, P_{O}=\right.$ $\left.5 d B ., \epsilon_{p}=0.1\right)$ in Fig.3.11 as the reference of PTPC case.

Similar to ON-OFF case, the secondary power budget significantly affects the throughput scaling. As noticed from Fig.3.10 and Fig.3.11, when $P_{a v}$ or $P_{O}$ is decreased, the throughput scaling is shifted downwards. Next, the decrement in $\epsilon_{p}^{0}$ makes the throughput drop because the trucated threshold $g_{T}=-\log \left(1-\epsilon_{p}^{0}\right)$ (in Rayleigh fading model) is reduced. Although the additional outage is raised like ON-OFF power policy, SBS will always stop transmitting if SBS decides to protect PU when PU is active in TCI case. Further, suppose that $\epsilon_{p, 1}^{0}>\epsilon_{p, 2}^{0}$, then $g_{T, 1}>g_{T, 2}$. With $\epsilon_{p}^{0}=\epsilon_{p, 2}^{0}$, SU can transmit when $g \in\left[g_{T, 2}, g_{T, 1}\right]$. However, the interference from PU to secondary network is really high in this additional range as 


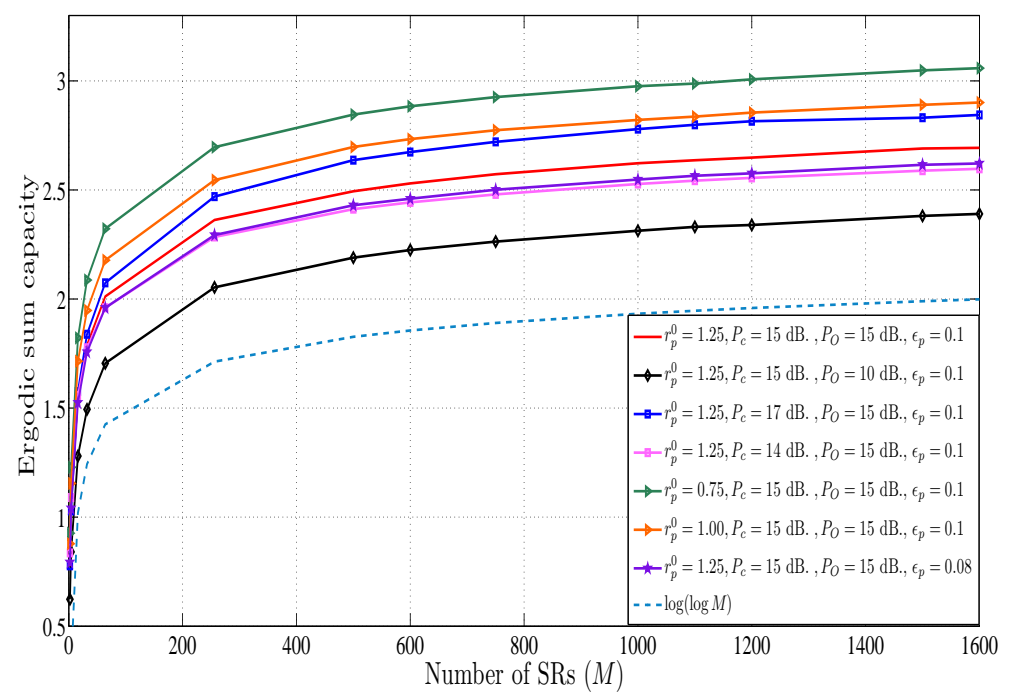

Figure 3.8: The effect of related variables on SBS sum throughput with POC against $M$ for ON-OFF power policy at PU and PTPC

as PU transmits with power $\frac{\left(e^{r_{p}^{0}}-1\right) N_{0}}{g}$. It means the range $g \in\left[g_{T, 2}, g_{T, 1}\right]$ does not help enhance secondary throughput much, making SU throughput scaling declines as $\epsilon_{p}^{0}$ reduces. Finally, $r_{p}^{0}$ affects the throughput scaling via POC as shown in Fig.3.10 and Fig.3.11 when $r_{p}^{0}$ becomes 4.00. The reason is that if the PU target rate is reduced, POC becomes more lenient, thereby making throughput scaling raise.

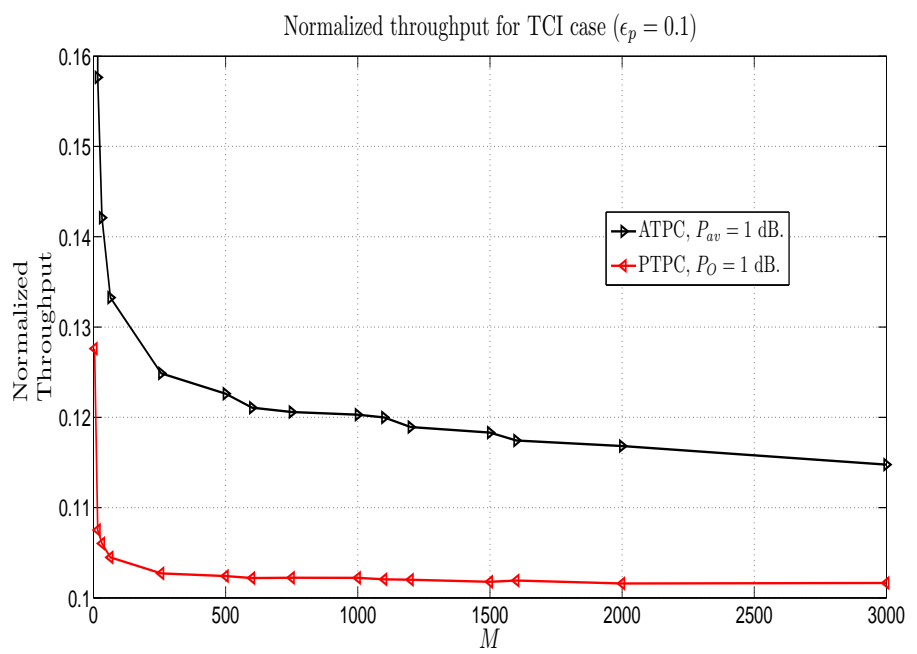

Figure 3.9: Normalized throughput in TCI power policy at the PU 


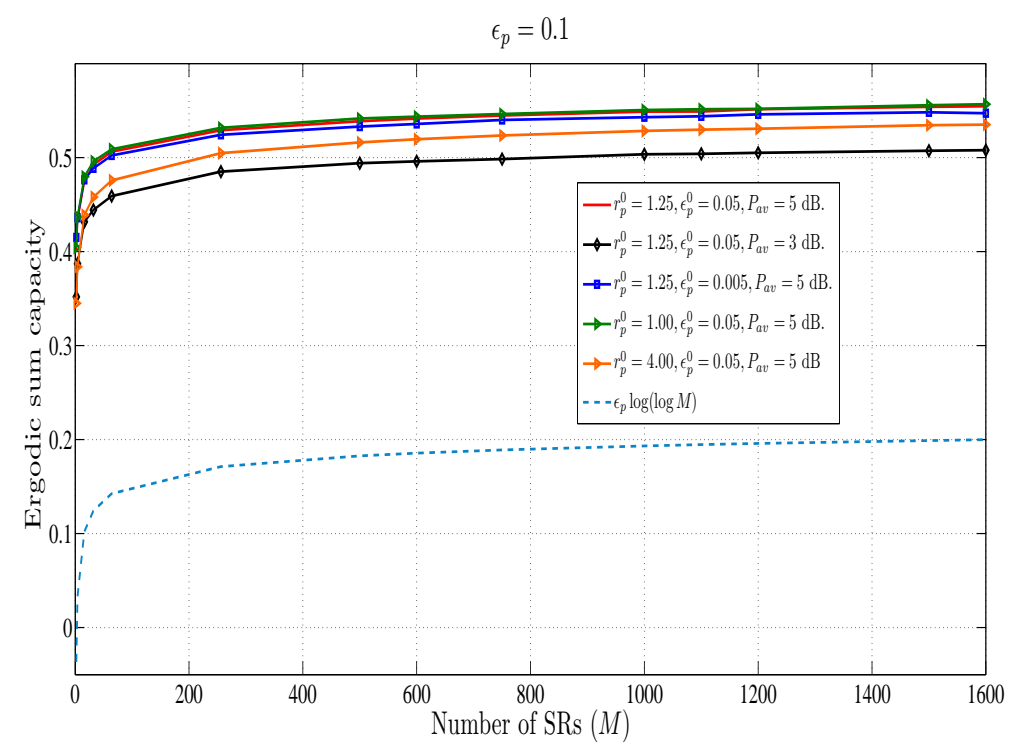

Figure 3.10: The effect of related variables on SU throughput scaling with POC against $M$ with TCI at PU. for ATPC

\subsection{Conclusion}

In this chapter, we have investigated the information-theoretic asymptotic limits of the ergodic sum capacity of a fading cognitive broadcast channel that shares the same frequency band as a delay sensitive PU. Under an outage probability constraint at the PU receiver, we have derived optimal power allocation strategies to maximize the SBS ergodic sum capacity, under an average (long term) transmit power constraint or a peak (short term) transmit power constraint. The derivation of the power policies is based on a probabilistic power allocation technique, which reveals that it is optimal to allocate transmission during an entire fading block to only one SR for a continuous fading channel scenario. Under these opportunistic SBS optimal power allocation schemes, we have also analyzed how the SBS sum throughput scales as the number of secondary receivers goes to infinity when all relevant channels undergo independent Rayleigh fading. These asymptotic capacity scaling laws are derived under two types of transmission power policies assumed at the PU: (1) an ONOFF policy with a constant power when ON and (2) a truncated channel inversion policy. Rigorous theoretical analyses show that the SBS sum throughput scales like $\log (\log M)$ and $\epsilon_{p} \log (\log M)$ for cases (1) and (2), respectively. Numerical results are also presented to illustrate the theoretical findings. 


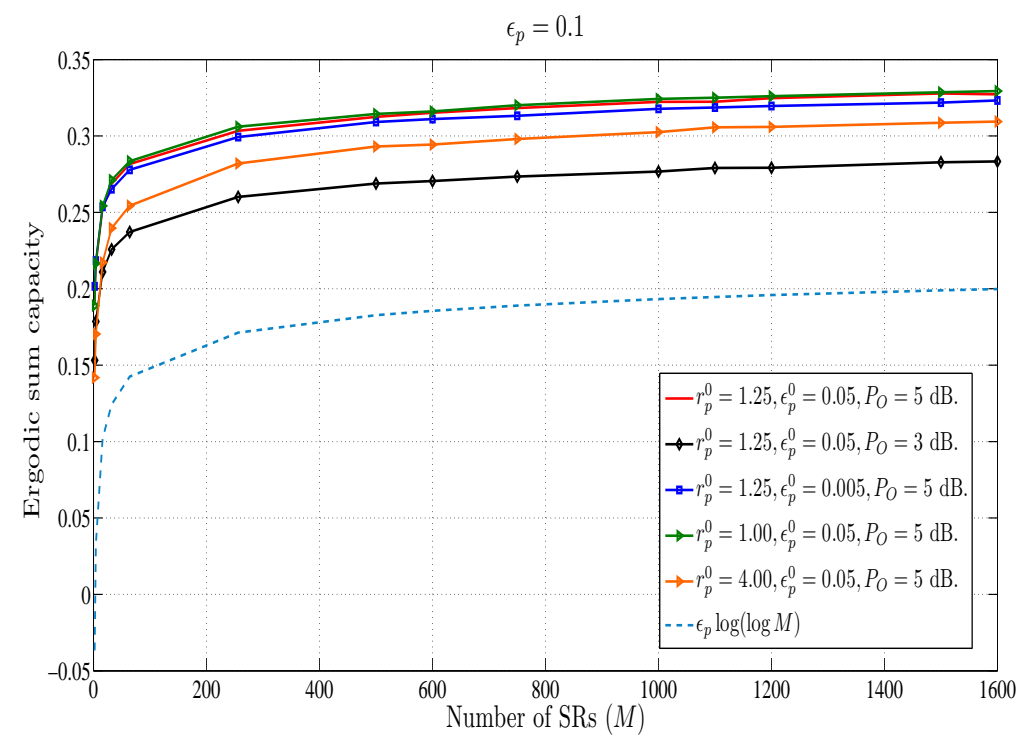

Figure 3.11: The effect of related variables on SU throughput scaling with POC against $M$ with TCI at PU. for PTPC 


\section{Chapter 4}

\section{Power Allocation in Cognitive Multiple-access Channels with Primary Outage Probability Constraint}

In Chapter 4, we consider SISO uplink channels in an underlay-based CR scenario with $M$ secondary transmitters and an SBS, sharing the same frequency band with a delay-sensitive primary user. This chapter investigates various optimal power allocation problems regarding two commonly adopted utility functions for fading channels, namely, the ergodic capacity [10] and outage capacity [21]. Ergodic capacity determines the maximum mutual information averaged over all the channel fading states regardless of a delay constraint while outage capacity defines the maximum instantaneous information rate that can be supported with a given outage probability and it is therefore a more appropriate performance indicator for delay-sensitive applications.

Information theoretic capacity notions for non-cognitive SISO fading multipleaccess channels were investigated in, e.g. [13,14,19,22]. For non-cognitive MAC, the authors in [13] showed that a given time slot (over which the fading channel remains invariant) is allocated to the secondary transmitter with the strongest channel power gain in order to maximize ergodic sum capacity. Under the same network setup considered in [13], Tse and Hanly studied two types of information theoretic capacity notions, i.e. ergodic capacity in [14] and delay-limited capacity (also known as zerooutage capacity) in [19]. In [14] and [19], the idea of polymatroid structure was used as the main ingredient to show that successive decoding is always optimal to achieve all boundary points on ergodic capacity and delay-limited capacity regions, leading to closed-form expressions of the optimal power policies. However, in [21] and [22], 
the authors argued that outage situation is unavoidable in some wireless channel models, e.g. Rayleigh fading, and zero-outage capacity is therefore unattainable. In [22], the authors defined two outage scenarios in non-cognitive MAC. The first one is known as common outage where an outage must be declared simultaneously for all users and the second one is individual outage where an outage can be declared individually. Significantly, it was shown in [22] that finding the outage capacity region is equivalent to deriving the outage probability region for a given rate vector. Similar to the results in [14] and [19], in [22], the authors also proved that successive decoding is the optimal decoding strategy. In the C-MAC scenario, [52] solved the ergodic sum capacity maximization problem subject to a transmit power constraint and an interference power constraint for both long-term and short-term perspectives in the single-input-single-output (SISO) case. Under the peak interference power constraints at PU receivers, the optimal power allocation policy for maximizing the weighted sum instantaneous rate of multi-antenna under the peak interference power constraints at PU receivers in C-MAC was later proposed in [84]. Recently, both the common outage capacity region and the individual outage capacity region for SUs in $\mathrm{C}-\mathrm{MAC}$ subject to peak interference power constraint and peak transmit power constraint were studied and the corresponding optimal power allocation strategies were solved in [53]. This chapter, on the contrary, utilizes POC as a mean to entrench the QoS of the single-antenna delay-sensitive PU.

This chapter considers fading C-MAC where $M$ SUs, equipped with a single antenna each, communicate with a secondary base station (SBS) by sharing the same spectrum band with an existing delay-sensitive PU. The service quality of PU is guaranteed by a primary outage probability constraint (POC). The power budget in each secondary transmitter is limited by an average transmit power constraint (ATPC). We assume that SUs have perfect CSI of the entire network while the PU has power policy based on the full CSI of its own direct gain between PU transmitter and PU receiver only and that strategy is revealed to all SUs. The novel contributions of this chapter can be summarized as the following:

\section{- Section 4.2 : Ergodic capacity}


1. In Section 4.2.1, we derive the optimal power control policy for the ergodic sum uplink capacity maximization problem subject to POC and ATPC, which is the special boundary point on the ergodic capacity region under POC and ATPC.

2. In Section 4.2.2, we extend the problem to the generalized case, i.e. we render the optimal power control policy to achieve any boundary points of the ergodic capacity region under POC and ATPC.

3. In Section 4.2.3, we thoroughly discuss the optimal power allocation results between the special case (ergodic sum capacity maximization) and the more generalized problem (ergodic capacity region). We show that the complicated optimal power solution in the more generalized case subsumes the optimal solution in the ergodic sum capacity case.

\section{- Section 4.3 : Outage capacity}

1. The definitions of common outage and individual outage are introduced in Section 4.3.1.

2. In Section 4.3.2, we study the common outage capacity region subject to POC and ATPC. Rather than solving the problem directly, we utilize a technique similar to [22] in order to show that the outage capacity region can be implicitly acquired by considering the usage probability maximization problem subject to POC and ATPC for a given SU rate vector $\mathbf{r}_{o}$ and then derive the optimal power policy accordingly.

3. In Section 4.3.3, we derive the optimal power policy that can achieve all boundary points on individual outage capacity region subject to POC and ATPC by following the same procedure as when we analyze the common outage capacity region subject to POC and ATPC

Simulation results are illustrated in Section 4.4 followed by concluding remarks in Section 4.5.

List of notations in Chapter 4: Here is the list of notations we use in this chapter. $E[$.$] denotes the statistical expectation. \operatorname{Pr}\{$.$\} represents probability. Cumulative$ 
density function $(\mathrm{CDF})$ of random variable $Z$ is given by $F_{Z}(z)$ whereas $F_{Z}(z \mid Y)$ expresses CDF of $Z$ given $Y$. Let $X_{w}$ be a Bernoulli $w$ random variable such that $X_{w}=1$ with probability $w$ and $X_{w}=0$ with probability $1-w \cdot \frac{\partial y}{\partial x^{*}}$ denotes partial derivative of $y$ with respect to $x$, evaluated at $x=x^{*} . \mathbf{P}^{T}$ represents transpose of vector $\mathbf{P}$. For two vectors $\mathbf{P}$ and $\mathbf{Q}, \mathbf{P Q}=\left\{P_{1} Q_{1}, \ldots, P_{M} Q_{M}\right\} .[x]^{+}=\max (0, x)$. $\mathbf{1}\{z\}$ represents the indicator function, i.e. $\mathbf{1}\{\mathscr{Z}\}=1$ if the event $\mathscr{Z}$ is true and it is zero otherwise.

\subsection{System model}

We consider a cognitive radio environment with a primary transmitter-receiver pair (PT-PR) and a cognitive multiple-access channel with an SBS and $M$ secondary transmitters. All terminals involved are equipped with a single antenna. The primary user's quality of service is guaranteed by a POC with a service rate $r_{p}^{0}$ and an outage probability threshold $\epsilon_{p}$. All channels involved in this cognitive radio network are assumed to be independent block fading additive white Gaussian noise (BF-AWGN) channels with continuous CDFs [21].

In the C-MAC, $M$ secondary transmitters aim to transmit the signal to the SBS. Let $\mathrm{ST}_{i}$ represent $i$-th secondary transmitter. As depicted in Figure 4.1, the instantaneous channel power gains in $i$-th subchannel for the link PT-PR, $\mathrm{ST}_{i}$-SBS, PT-SBS, and $\mathrm{ST}_{i}$-PR are denoted by $g, h_{i}, \alpha$, and $\beta_{i}$. The additive noises at PR and SBS are assumed to be independent Gaussian random variables with zero mean and variance $N_{0}$. Let $\boldsymbol{\nu}=\left[g, \alpha, h_{1}, \ldots, h_{M}, \beta_{1}, \ldots, \beta_{M}\right]^{T}$ denote the combined channel state vector and $\boldsymbol{\beta}=\left[\beta_{1}, \ldots, \beta_{M}\right]^{T}$. The vector fading process $\boldsymbol{\nu}$ is assumed to be stationary and ergodic, with a continuous CDF $F(\boldsymbol{\nu})$. We assume the primary user's transmit power control strategy is based only on the direct gain $g$ between PT and $\mathrm{PR}$, regardless of the interference from the secondary network. We also assume that SBS has perfect CSI on $\boldsymbol{\nu}$ and primary user's power policy, so that it also knows the PU's power allocation for every realization of $\boldsymbol{\nu}$. Let $\mathbf{P}(\boldsymbol{\nu})=\left[P_{1}(\boldsymbol{\nu}), \cdots, P_{M}(\boldsymbol{\nu})\right]^{T}$ which denotes the $i$-th ST's transmit power to the SBS. The PU instantaneous rate 
expression can be written as

$$
r_{p}(\boldsymbol{\nu}, \mathbf{P}(\boldsymbol{\nu}))=\log \left(1+\frac{g P_{p}(g)}{\left(\sum_{i=1}^{M} \beta_{i} P_{i}(\boldsymbol{\nu})\right)+N_{0}}\right)
$$

Note that PU's power control policy is expressed as $P_{p}(g)$ to reflect the PU's obliviousness to the interference caused by the secondary network and having the power policy based on CSI of its direct gain $g$ only. In a typical wireless fading environment, even in the absence of interference from the secondary network, the PU may not be able to avoid an outage event when the PT-PR channel is in deep fade. For example, with an average or peak transmit power constraint, the PU cannot meet the target rate for a Rayleigh fading channel if it falls below a certain threshold [21] unless it is equipped with multiple antennas. We further presume that the PU's power policy is designed to allow for a maximum $\mathrm{PU}$ outage probability of $\epsilon_{p}^{0} \leq \epsilon_{p}$ in the absence of the secondary interference. In this chapter, PT is assumed to employ an $O N$ - OFF power strategy with constant power when ON (ON-OFF), i.e. the PU transmits with constant power $P_{p}(g)=P_{c}$ when $g \geq g_{T}=\frac{\left(e^{r_{p}^{0}}-1\right) N_{0}}{P_{c}}$ to meet the target rate and $P_{p}(g)=0$ for $g<g_{T}$, where $N_{0}$ denotes the AWGN variance at the primary receiver.

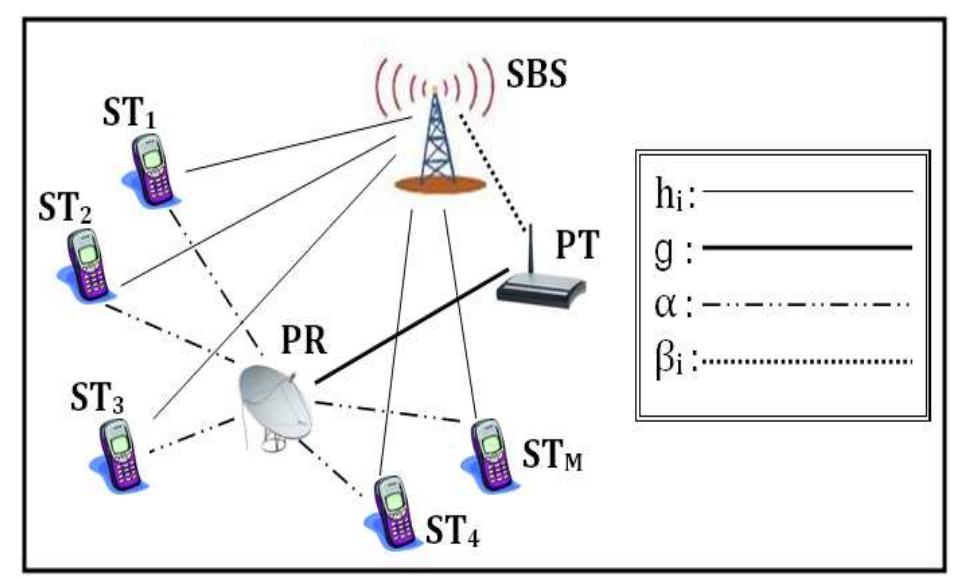

Figure 4.1: System model for cognitive MAC

Define $\mathbf{P}_{a v}=\left[P_{a v, 1}, \ldots, P_{a v, M}\right]^{T}$ where $P_{a v, i}$ is the average transmit power budget at the $i$-th ST. The delay-sensitive primary network requires a target rate $r_{p}^{0}$ 
and a maximum outage probability threshold $\epsilon_{p}$. The average transmit power constraint (ATPC) and the primary outage probability constraint (POC) are described as follows:

$$
\begin{gathered}
E[\mathbf{P}(\boldsymbol{\nu})] \preceq \mathbf{P}_{a v} \\
\operatorname{Pr}\left\{r_{p}(\boldsymbol{\nu}, \mathbf{P}(\boldsymbol{\nu}))<r_{p}^{0}\right\} \leq \epsilon_{p}
\end{gathered}
$$

For convenience, define $\bar{h}_{i}=\frac{h_{i}}{\alpha P_{p}(g)+N_{0}}$. With a given power allocation $\mathbf{P}(\boldsymbol{\nu})$ in a specific channel state $\boldsymbol{\nu}$ satisfying ATPC and POC, the following rate vectors are achievable in this given channel state $\boldsymbol{\nu}$

$$
\mathcal{C}_{M A C}(\boldsymbol{\nu}, \mathbf{P}(\boldsymbol{\nu}))=\left\{\mathbf{r}=\left[r_{1}, \ldots, r_{M}\right]: \sum_{i \in S} r_{i} \leq \log \left(1+\sum_{i \in S} \bar{h}_{i} P_{i}(\boldsymbol{\nu})\right), \forall S \subset\{1, \ldots, M\}\right\}
$$

The definition of $\mathcal{C}_{M A C}(\boldsymbol{\nu}, \mathbf{P}(\boldsymbol{\nu}))$ in (4.4) is the capacity region of the equivalent Gaussian MAC for the channel state $\boldsymbol{\nu}$, corresponding to the power control $\mathbf{P}(\boldsymbol{\nu})$ in the secondary network.

\subsection{Ergodic capacity region}

In this part, we will investigate the ergodic capacity region for C-MAC under ATPC and POC. First, we look at the result for optimal power allocation policy in a special case - ergodic sum rate maximization problem - in Section 4.2.1, i.e. when $S=\{1, \ldots, M\}$. Note that when $M=1$, i.e. the system is reduced from cognitive MAC to cognitive point-to-point communication, the solution has already appeared in [44]. Later in Section 4.2.2, we will study the more general case for all possible subsets $S$ of the set $\mathcal{E}=\{1, \ldots, M\}$, i.e. the optimal power allocation policy that achieves all points in the boundary of the ergodic capacity region. 


\subsubsection{Special case: Ergodic sum rate maximization problem with ATPC and POC}

For the instantaneous sum rate, we have $S=\mathcal{E}$. Hence from (4.4), the instantaneous sum uplink capacity in C-MAC can be described as follows:

$$
r_{s}(\boldsymbol{\nu}, \mathbf{P}(\boldsymbol{\nu}))=\log \left(1+\sum_{i=1}^{M} \bar{h}_{i} P_{i}(\boldsymbol{\nu})\right)
$$

This section will focus on the ergodic sum capacity maximizing problem in C-MAC with ATPCs and POC which can be expressed as

$$
\max _{\mathbf{P}(\boldsymbol{\nu}) \succeq 0} E\left[r_{s}(\boldsymbol{\nu}, \mathbf{P}(\boldsymbol{\nu}))\right] \quad \text { s.t. (4.2) and (4.3). }
$$

With the same technique as in [25], we define the two weighting functions $w_{k}(\boldsymbol{\nu}), k=$ 1,2 as follows:

$$
\begin{array}{ll}
w_{1}(\boldsymbol{\nu})= & \operatorname{Pr}\left\{r_{p}(\boldsymbol{\nu}, \mathbf{P}(\boldsymbol{\nu})) \geq r_{p}^{0} \mid \boldsymbol{\nu}\right\} \\
w_{2}(\boldsymbol{\nu})= & \operatorname{Pr}\left\{r_{p}(\boldsymbol{\nu}, \mathbf{P}(\boldsymbol{\nu}))<r_{p}^{0} \mid \boldsymbol{\nu}\right\}
\end{array}
$$

It is obvious to see that $w_{2}(\boldsymbol{\nu})=1-w_{1}(\boldsymbol{\nu})$ and we will use this fact from now on. We also define the two corresponding power schemes as follows:

$$
\begin{array}{ll}
\mathbf{p}_{1}(\boldsymbol{\nu})= & E\left[\mathbf{P}(\boldsymbol{\nu}) \mid r_{p}(\boldsymbol{\nu}, \mathbf{p}(\boldsymbol{\nu})) \geq r_{p}^{0}, \boldsymbol{\nu}\right] \\
\mathbf{p}_{2}(\boldsymbol{\nu})= & E\left[\mathbf{P}(\boldsymbol{\nu}) \mid r_{p}(\boldsymbol{\nu}, \mathbf{p}(\boldsymbol{\nu}))<r_{p}^{0}, \boldsymbol{\nu}\right]
\end{array}
$$

The definitions of $w_{1}(\boldsymbol{\nu}), \mathbf{p}_{1}(\boldsymbol{\nu})$ and $\mathbf{p}_{2}(\boldsymbol{\nu})$ above lead to Lemma 4.2 .1 below, the proof of which is provided in Appendix C.1.

Lemma 4.2.1. There exists an optimum solution $\mathbf{P}^{*}(\boldsymbol{\nu})$ of Problem (4.6) of the form $\mathbf{P}^{*}(\boldsymbol{\nu})=X_{w_{1}^{*}(\boldsymbol{\nu})}(\boldsymbol{\nu}) \mathbf{p}_{1}^{*}(\boldsymbol{\nu})+\left(1-X_{w_{1}^{*}(\boldsymbol{\nu})}(\boldsymbol{\nu})\right) \mathbf{p}_{2}^{*}(\boldsymbol{\nu})$ where

- (1) $E\left[w_{1}^{*}(\boldsymbol{\nu}) \mathbf{p}_{1}^{*}(\boldsymbol{\nu})+\left(1-w_{1}^{*}(\boldsymbol{\nu})\right) \mathbf{p}_{2}^{*}(\boldsymbol{\nu})\right] \preceq \mathbf{P}_{a v}$,

(2) $E\left[w_{1}^{*}(\boldsymbol{\nu})\right] \geq 1-\epsilon_{p}$

(3) $w_{1}^{*}(\boldsymbol{\nu})\left[\left(\frac{g P_{p}(g(\boldsymbol{\nu}))}{e^{r 0}-1}-N_{0}\right)^{+}-\boldsymbol{\beta}^{T} \mathbf{p}_{1}^{*}(\boldsymbol{\nu})\right] \geq 0, \quad$ (4) $\mathbf{p}_{k}^{*}(\boldsymbol{\nu}) \succeq \mathbf{0}, \quad k=1,2$, (5) $0 \leq w_{1}^{*}(\boldsymbol{\nu}) \leq 1$ 
Then, the optimization problem in (4.6) can be rewritten as

$$
\begin{array}{cl}
\max _{\mathbf{p}_{k}(\boldsymbol{\nu}) \succeq \mathbf{0}, w_{1}(\boldsymbol{\nu})} & R_{s}=E\left[w_{1}(\boldsymbol{\nu}) r_{s}\left(\boldsymbol{\nu}, \mathbf{p}_{1}(\boldsymbol{\nu})\right)+\left(1-w_{1}(\boldsymbol{\nu})\right) r_{s}\left(\boldsymbol{\nu}, \mathbf{p}_{2}(\boldsymbol{\nu})\right)\right] \\
\text { s.t. } & E\left[w_{1}(\boldsymbol{\nu}) \mathbf{p}_{1}(\boldsymbol{\nu})+\left(1-w_{1}(\boldsymbol{\nu})\right) \mathbf{p}_{2}(\boldsymbol{\nu})\right] \preceq \mathbf{P}_{a v}, \\
& E\left[w_{1}(\boldsymbol{\nu})\right] \geq 1-\epsilon_{p}, \\
& w_{1}(\boldsymbol{\nu})\left[\left(\frac{g P_{p}(g)}{e^{r_{p}^{0}}-1}-N_{0}\right)^{+}-\boldsymbol{\beta}^{T} \mathbf{p}_{1}(\boldsymbol{\nu})\right] \geq 0, \\
& 0 \leq w_{1}(\boldsymbol{\nu}) \leq 1 .
\end{array}
$$

The optimization problem (4.9) can be proved to be convex over $\left(\mathbf{p}_{1}(\boldsymbol{\nu}), \mathbf{p}_{2}(\boldsymbol{\nu}), w_{1}(\boldsymbol{\nu})\right)$. We can then write the Lagrangian associated with (4.9) as follows:

$$
\begin{aligned}
& l\left(\boldsymbol{\nu}, \mathbf{p}_{k}(\boldsymbol{\nu}), w_{1}(\boldsymbol{\nu}), \boldsymbol{\lambda}, s, \tau(\boldsymbol{\nu})\right) \\
& =w_{1}(\boldsymbol{\nu})\left[r_{s}\left(\boldsymbol{\nu}, \mathbf{p}_{1}(\boldsymbol{\nu})\right)-\sum_{i=1}^{M} \lambda_{i} p_{1, i}(\boldsymbol{\nu})+s+\tau(\boldsymbol{\nu})\left(\mathcal{P}_{p}(g)-\sum_{i=1}^{M} p_{1, i}(\boldsymbol{\nu}) \beta_{i}\right)\right] \\
& \quad+\left(1-w_{1}(\boldsymbol{\nu})\right)\left[r_{s}\left(\boldsymbol{\nu}, \mathbf{p}_{2}(\boldsymbol{\nu})\right)-\sum_{i=1}^{M} \lambda_{i} p_{2, i}(\boldsymbol{\nu})\right]
\end{aligned}
$$

where $\boldsymbol{\lambda}, s$, and $\tau(\boldsymbol{\nu})$ are Lagrange multipliers corresponding to equations (4.9b) to (4.9d). Let $i \in\{1,2, \ldots, M\}$ and $k \in\{1,2\}$. The set of KKT conditions corresponding to (4.9) are provided in Appendix C.2.

Using the KKT conditions, $\mathbf{p}_{2}^{*}(\boldsymbol{\nu}), \mathbf{p}_{1}^{*}(\boldsymbol{\nu})$, and $w_{1}^{*}(\boldsymbol{\nu})$ can be derived as follows:

- For $p_{2, i}^{*}(\boldsymbol{\nu})$, we have $0=\frac{\partial l(\ldots)}{\partial p_{2, i}^{*}(\boldsymbol{\nu})}=\left(1-w_{1}^{*}(\boldsymbol{\nu})\right)\left[\frac{\partial r_{s}\left(\boldsymbol{\nu}, \mathbf{p}_{2}(\boldsymbol{\nu})\right)}{\partial p_{2, i}^{*}(\boldsymbol{\nu})}-\lambda_{i}^{*}\right]$ if $p_{2, i}^{*}(\boldsymbol{\nu})>0$. If $1-w_{1}^{*}(\boldsymbol{\nu})>0$, it yields $1+\sum_{i=1}^{M} p_{2, i}^{*}(\boldsymbol{\nu}) \bar{h}_{i}=\left(\frac{\bar{h}_{i}}{\lambda_{i}^{*}}\right)$. Let $p_{w f, i}^{*}(\boldsymbol{\nu})=\left(\frac{1}{\lambda_{i}^{*}}-\frac{1}{h_{i}}\right)^{+}$. By using the fact that $\lambda_{i}^{*}$ is constant for all $i$ and the channel state is continuous, $p_{2, i}^{*}(\boldsymbol{\nu})$ can be expressed as follows:

$$
p_{2, i}^{*}(\boldsymbol{\nu})= \begin{cases}p_{w f, i}^{*}(\boldsymbol{\nu}) & , w_{1}^{*}(\boldsymbol{\nu})=0, i=\arg \max _{m \in \mathcal{E}} \frac{\bar{h}_{m}}{\lambda_{m}^{*}} \\ 0 & , \text { otherwise }\end{cases}
$$

Obviously, the optimal power allocation policy $\mathbf{p}_{2}^{*}$ is exactly the same as the optimal power allocation policy that maximizes the ergodic sum capacity for non-cognitive uplink channels, previously studied in $[13,14]$. Intuitively, this 
happens because $\mathbf{p}_{2}^{*}$ is used when the secondary network decides to put the $\mathrm{PU}$ in outage and thus does not have to control the amount of interference caused to the primary link.

- For $p_{1, i}^{*}(\boldsymbol{\nu})$, we have $\frac{\partial l(\ldots)}{\partial p_{1, i}^{*}(\boldsymbol{\nu})}=w_{1}^{*}(\boldsymbol{\nu})\left[\frac{\partial r_{s}\left(\boldsymbol{\nu}, \mathbf{p}_{1}^{*}(\boldsymbol{\nu})\right)}{\partial p_{1, i}^{*}(\boldsymbol{\nu})}-\lambda_{i}^{*}-\tau^{*}(\boldsymbol{\nu}) \beta_{i}\right]$ from the KKT conditions.

Note that in using the policy $\mathbf{p}_{1}^{*}(\boldsymbol{\nu})$ when $w_{1}^{*}(\boldsymbol{\nu})>0$, our problem is similar to the sub-dual problem with peak interference constraint for each given channel state $\boldsymbol{\nu}$. This implication is based on the fact that $\mathcal{P}_{p}(g)=\boldsymbol{\beta}^{T} \mathbf{p}_{1}^{*}(\boldsymbol{\nu})$ if $\tau^{*}(\boldsymbol{\nu})>$ 0 . Therefore, it is obvious that the solution for $\mathbf{p}_{1}^{*}$ is similar to $\mathbf{p}_{2}^{*}$ for $\tau^{*}(\boldsymbol{\nu})=0$. For $\tau^{*}(\boldsymbol{\nu})>0$, it implies that $1+\sum_{i=1}^{M} p_{1, i}^{*}(\boldsymbol{\nu}) \bar{h}_{i}=\left(\frac{\bar{h}_{i}}{\lambda_{i}^{*}+\tau^{*}(\boldsymbol{\nu}) \beta_{i}}\right)$ if $p_{1, i}^{*}(\boldsymbol{\nu})>0$.

Lemma 4.2.2. For $\mathbf{p}_{1}^{*}(\boldsymbol{\nu})$, there are at most two users that are allowed to transmit

Proof. Suppose there are $|\mathscr{V}|$ users with $p_{1, v}^{*}>0$, where $v \in \mathscr{V}$ and $\mathscr{V} \subset \mathcal{E}$. The condition $1+\sum_{i=1}^{M} p_{1, i}^{*}(\boldsymbol{\nu}) \bar{h}_{i}=\left(\frac{\bar{h}_{v}}{\lambda_{v}^{*}+\tau^{*}(\boldsymbol{\nu}) \beta_{v}}\right)<\left(\frac{\bar{h}_{v}}{\lambda_{v}^{*}}\right)$ must hold for all $v \in \mathscr{V}$. Note that there is only one $\tau^{*}(\boldsymbol{\nu})$, but $|\mathscr{V}|-1$ independent equations. Hence, $1 \geq|\mathscr{V}|-1$. So, there are at most two users can transmit when $u^{*}(\boldsymbol{\nu})>0$.

Thus, the analysis is divided into two cases, i.e. when one user transmits and when two users transmit.

\section{Only one user transmits :}

$$
p_{1, i}^{*}(\boldsymbol{\nu})= \begin{cases}p_{w f, i}^{*}(\boldsymbol{\nu}) & , w_{1}^{*}(\boldsymbol{\nu})=1, \tau^{*}(\boldsymbol{\nu})=0, i=\arg \max _{m \in \mathcal{E}} \frac{h_{m}}{\lambda_{m}^{*}} \\ p_{r p, i}^{*}(\boldsymbol{\nu}) & , w_{1}^{*}(\boldsymbol{\nu})=1, \tau^{*}(\boldsymbol{\nu})>0, i=\arg \max _{m \in \mathcal{E}} \frac{\bar{h}_{i}}{\lambda_{i}^{*}+u^{*}(\boldsymbol{\nu}) \beta_{i}} \\ 0 & , \text { otherwise }\end{cases}
$$

where $p_{r p, i}^{*}(\boldsymbol{\nu})=\frac{1}{\beta_{i}} \mathcal{P}_{p}(g)$.

\section{Two users transmit :}

If there are two active SUs, i.e. $p_{1, b}^{*}, p_{1, v}^{*}>0$ and $b \neq v$, the two following 
conditions in (4.13) and (4.14) must hold

$$
\begin{aligned}
& 1+\sum_{i \in \mathcal{E}} p_{1, i}^{*}(\boldsymbol{\nu}) \bar{h}_{i}=1+p_{1, v}^{*}(\boldsymbol{\nu}) \bar{h}_{v}+p_{1, b}^{*}(\boldsymbol{\nu}) \bar{h}_{b}=\frac{\frac{\bar{h}_{v}}{\beta_{v}}-\frac{\bar{h}_{b}}{\beta_{b}}}{\frac{\lambda_{v}}{\beta_{v}}-\frac{\lambda_{b}}{\beta_{b}}} \\
& \sum_{i \in \mathcal{E}} p_{1, i}^{*}(\boldsymbol{\nu}) \beta_{i}=p_{1, v}^{*}(\boldsymbol{\nu}) \beta_{v}+p_{1, b}^{*}(\boldsymbol{\nu}) \beta_{b} \quad=\mathcal{P}_{p}(g)
\end{aligned}
$$

Note that $(4.13)$ is due to $\tau^{*}(\boldsymbol{\nu})=\frac{1}{\beta_{v}}\left(\frac{\bar{h}_{v}}{1+\sum_{i \in \mathcal{E}} p_{1, i}^{*}(\boldsymbol{\nu}) \bar{h}_{i}}-\lambda_{v}^{*}\right)=\frac{1}{\beta_{b}}\left(\frac{\bar{h}_{b}}{1+\sum_{i \in \mathcal{E}} p_{1, i}^{*}(\boldsymbol{\nu}) \bar{h}_{i}}-\lambda_{b}^{*}\right)$. (4.14) follows from the KKT conditions.

Hence, we can summarize the solution for this case as follows:

$$
p_{1, i}^{*}(\boldsymbol{\nu})=\left\{\begin{aligned}
p_{w f, i}^{*}(\boldsymbol{\nu}), & w_{1}^{*}(\boldsymbol{\nu})=1, \tau^{*}(\boldsymbol{\nu})=0, \quad\left(i=\underset{m \in \mathcal{E}}{\arg \max _{m} \frac{h_{m}}{\lambda_{m}^{*}}}\right) \\
p_{1, b}^{*}(\boldsymbol{\nu}) \quad, w_{1}^{*}(\boldsymbol{\nu})=1, \tau^{*}(\boldsymbol{\nu})>0, & \\
& \left(i \in\left\{v, b \mid v, b=\arg \max _{m \in \mathcal{E}} \frac{\bar{h}_{i}}{\lambda_{i}^{*}+\tau^{*}(\boldsymbol{\nu}) \beta_{i}}\right\}\right), \\
& \left(p_{1, b}^{*}(\boldsymbol{\nu}) \text { satisfies }(4.13) \operatorname{and}(4.14)\right) \\
, & w_{1}^{*}(\boldsymbol{\nu})=1, \tau^{*}(\boldsymbol{\nu})>0, \\
& \left(i \in\left\{v, b \mid v, b=\arg \max _{m \in \mathcal{E}} \frac{\bar{h}_{i}}{p_{i, v}^{*}(\boldsymbol{\nu}) \tau^{*}(\boldsymbol{\nu}) \beta_{i}}\right\}\right) \\
& \left(p_{1, v}^{*}(\boldsymbol{\nu}) \text { satisfies }(4.13) \text { and }(4.14)\right) \\
, & \text { otherwise }
\end{aligned}\right.
$$

Remark 4.2.1. To search for $p_{1, i}^{*}(\boldsymbol{\nu})>0$, the geometrical method proposed in [85] can be applied. Note that the optimal solution $p_{1, i}^{*}(\boldsymbol{\nu})$ does not have a closed-form expression when two users are allowed to transmit.

Remark 4.2.2. Despite allowing two users to transmit, the expression of the achievable sum rate is still the same, i.e. $\log \left(1+\sum_{i=1}^{M} p_{1, i}^{*}(\boldsymbol{\nu}) \bar{h}_{i}\right)=\log \left(\frac{\bar{h}_{v}}{\lambda_{v}^{*}+\tau^{*}(\boldsymbol{\nu}) \beta_{v}}\right)$.

- For $w_{1}^{*}(\boldsymbol{\nu})$, we first define two benefit functions as follows: $\mathscr{B}_{1}\left(\boldsymbol{\nu}, \mathbf{p}_{1}^{*}(\boldsymbol{\nu}), \boldsymbol{\lambda}^{*}, s^{*}\right)=$ $\mathscr{B}_{1, \boldsymbol{\nu}}=r_{s}\left(\boldsymbol{\nu}, \mathbf{p}_{1}^{*}(\boldsymbol{\nu})\right)-\boldsymbol{\lambda}^{* T} \mathbf{p}_{1}^{*}(\boldsymbol{\nu})+s^{*}$ and $\mathscr{B}_{2}\left(\boldsymbol{\nu}, \mathbf{p}_{2}^{*}(\boldsymbol{\nu}), \boldsymbol{\lambda}^{*}\right)=\mathscr{B}_{2, \boldsymbol{\nu}}=r_{s}\left(\boldsymbol{\nu}, \mathbf{p}_{2}^{*}(\boldsymbol{\nu})\right)-$ $\lambda^{* T} \mathbf{p}_{2}^{*}(\boldsymbol{\nu})$. The physical interpretation of benefit functions represents the profit 
due to using the power policy $\mathbf{p}_{k}^{*}(\boldsymbol{\nu})$. From (C.6), we get

$$
w_{1}^{*}(\boldsymbol{\nu})= \begin{cases}1, & \mathscr{B}_{1, \boldsymbol{\nu}}>\mathscr{B}_{2, \boldsymbol{\nu}} \\ 0, & \mathscr{B}_{1, \boldsymbol{\nu}}<\mathscr{B}_{2, \boldsymbol{\nu}}\end{cases}
$$

Note that $w_{1}^{*}(\boldsymbol{\nu})$ can take values either 1 or 0 for each given state $\boldsymbol{\nu}$ in the continuous fading channel case. The interpretation is that the secondary network will use policy $\mathbf{p}_{1}^{*}(\boldsymbol{\nu})$ if the policy 1 returns higher profit than policy 2 .

Thus, the optimal solution for the problem (4.6) can be summarized in Theorem 4.2.1 as follows:

Theorem 4.2.1. The optimal power control for (4.6) is given by $\mathbf{P}^{*}(\boldsymbol{\nu})=X_{w_{1}^{*}}(\boldsymbol{\nu}) \mathbf{p}_{1}^{*}+$ $\left(1-X_{w_{1}^{*}}(\boldsymbol{\nu})\right) \mathbf{p}_{2}^{*}$, where the expression of $\mathbf{p}_{1}^{*}$ is defined by either (4.12) or (4.15), the expression of $\mathbf{p}_{2}^{*}$ is defined by (4.11) and the expression of $w_{1}^{*}(\boldsymbol{\nu})$ follows from (4.16). The Lagrange multipliers $\boldsymbol{\lambda}^{*}$ and $s^{*}$ are the solutions to $E\left[\mathbf{P}^{*}(\boldsymbol{\nu})\right] \preceq \mathbf{P}_{a v}$ and $E\left[w_{1}^{*}(\boldsymbol{\nu})\right] \geq 1-\epsilon_{p}$.

\subsubsection{General case: Ergodic Capacity Region for Secondary Network with ATPC and POC}

In this part, we extend the result in Section 4.2.1 to the more generalized case, i.e. we consider all possible subsets $S$ of $\mathcal{E}$, by solving the optimal power policy to achieve all the boundary points of the ergodic capacity region with POC and ATPC. Thus, we have to consider all possibilities of the subsets $S \subset \mathcal{E}$. To this end, we apply the result in [14] which revealed that the capacity region of the fading MAC can be written as the weighted sum of the capacity regions of $\mathcal{C}_{M A C}(\boldsymbol{\nu}, \mathbf{P}(\boldsymbol{\nu}))$. Therefore, the problem is formulated as follows:

$$
\begin{array}{ll}
\max _{\mathbf{r}(\boldsymbol{\nu}, \mathbf{P}(\boldsymbol{\nu})), \mathbf{P}(\boldsymbol{\nu}) \succeq \mathbf{0}} & \boldsymbol{\mu}^{T} \mathbf{C}_{s}=\boldsymbol{\mu}^{T} E[\mathbf{r}(\boldsymbol{\nu}, \mathbf{P}(\boldsymbol{\nu}))] \\
\text { s.t. } & \mathbf{r}(\boldsymbol{\nu}, \mathbf{P}(\boldsymbol{\nu})) \in \mathcal{C}_{M A C}(\boldsymbol{\nu}, \mathbf{P}(\boldsymbol{\nu})),
\end{array}
$$

(4.2) and (4.3) 
Remark 4.2.3. It is worth noting that we also have to search for the optimal $\mathbf{r}(\boldsymbol{\nu}, \mathbf{P}(\boldsymbol{\nu}))$ in this problem as all combinations of $S$ are considered and the expression of $r_{i}(\boldsymbol{\nu}, \mathbf{P}(\boldsymbol{\nu}))$ in the rate vector $\mathbf{r}(\boldsymbol{\nu}, \mathbf{P}(\boldsymbol{\nu}))$ is affected by the decoding order. In [14], it was proved that because of the polymatroid structure of $\mathcal{C}_{M A C}(\boldsymbol{\nu}, \mathbf{P}(\boldsymbol{\nu}))$, for any power control $\mathbf{P}(\boldsymbol{\nu}), \boldsymbol{\mu}^{T} \mathbf{r}$ is maximized at

$$
\begin{aligned}
& R_{\pi(1)}=\log \left(1+\bar{h}_{\pi(1)} P_{\pi(1)}(\boldsymbol{\nu})\right) \\
& R_{\pi(k)}=\log \left(1+\frac{\bar{h}_{\pi(1)} P_{\pi(1)}(\boldsymbol{\nu})}{1+\sum_{i=1}^{k-1} \bar{h}_{\pi(i)} P_{\pi(i)}(\boldsymbol{\nu})}\right), \quad k=2, \cdots, M
\end{aligned}
$$

where $\pi$ is the permutation corresponding to a decreasing ordering of the components of the vector $\boldsymbol{\mu}$.

With similar procedure in Section 4.2.1, we first define $w_{1}(\boldsymbol{\nu}), \mathbf{p}_{1}(\boldsymbol{\nu})$ and $\mathbf{p}_{2}(\boldsymbol{\nu})$

$$
\begin{array}{cc}
w_{1}(\boldsymbol{\nu})= & \operatorname{Pr}\left\{r_{p}(\boldsymbol{\nu}, \mathbf{P}(\boldsymbol{\nu})) \geq r_{p}^{0} \mid \mathbf{r} \in \mathcal{C}_{M A C}(\boldsymbol{\nu}, \mathbf{P}(\boldsymbol{\nu})), \boldsymbol{\nu}\right\} \\
\mathbf{p}_{1}(\boldsymbol{\nu})= & E\left[\mathbf{P}(\boldsymbol{\nu}) \mid r_{p}(\boldsymbol{\nu}, \mathbf{p}(\boldsymbol{\nu})) \geq r_{p}^{0}, \mathbf{r} \in \mathcal{C}_{M A C}(\boldsymbol{\nu}, \mathbf{p}(\boldsymbol{\nu})), \boldsymbol{\nu}\right] \\
\mathbf{p}_{2}(\boldsymbol{\nu})= & E\left[\mathbf{P}(\boldsymbol{\nu}) \mid r_{p}(\boldsymbol{\nu}, \mathbf{p}(\boldsymbol{\nu}))<r_{p}^{0}, \mathbf{r} \in \mathcal{C}_{M A C}(\boldsymbol{\nu}, \mathbf{p}(\boldsymbol{\nu})), \boldsymbol{\nu}\right]
\end{array}
$$

For convenience, we further define $\mathbf{r}_{1}=\mathbf{r}\left(\boldsymbol{\nu}, \mathbf{p}_{1}(\boldsymbol{\nu})\right)$ and $\mathbf{r}_{2}=\mathbf{r}\left(\boldsymbol{\nu}, \mathbf{p}_{2}(\boldsymbol{\nu})\right)$. With $w_{1}(\boldsymbol{\nu}), \mathbf{p}_{1}(\boldsymbol{\nu}), \mathbf{p}_{2}(\boldsymbol{\nu}), \mathbf{r}_{1}$ and $\mathbf{r}_{2}$, we can prove the following Lemma:

Lemma 4.2.3. There exists an optimum solution $\mathbf{P}^{*}(\boldsymbol{\nu})$ of problem (4.20) of the form $\mathbf{P}^{*}(\boldsymbol{\nu})=X_{w_{1}^{*}(\boldsymbol{\nu})}(\boldsymbol{\nu}) \mathbf{p}_{1}^{*}(\boldsymbol{\nu})+\left(1-X_{w_{1}^{*}(\boldsymbol{\nu})}(\boldsymbol{\nu})\right) \mathbf{p}_{2}^{*}(\boldsymbol{\nu})$ where

$$
\begin{aligned}
& \text { - (1) } E\left[w_{1}^{*}(\boldsymbol{\nu}) \mathbf{p}_{1}^{*}(\boldsymbol{\nu})+\left(1-w_{1}^{*}(\boldsymbol{\nu})\right) \mathbf{p}_{2}^{*}(\boldsymbol{\nu})\right] \preceq \mathbf{P}_{a v}, \quad \text { (2) } E\left[w_{1}^{*}(\boldsymbol{\nu})\right] \geq 1-\epsilon_{p}, \\
& \begin{array}{ll}
\text { (3) } w_{1}^{*}(\boldsymbol{\nu})\left[\mathcal{P}_{p}(g)-\boldsymbol{\beta}^{T} \mathbf{p}_{1}^{*}(\boldsymbol{\nu})\right] \geq 0, & \text { (4) } \mathbf{p}_{k}^{*}(\boldsymbol{\nu}) \succeq \mathbf{0}, \quad k=1,2 \\
\text { (5) } 0 \leq w_{1}^{*}(\boldsymbol{\nu}) \leq 1, & \text { (6) } \mathbf{r}_{k} \in \mathcal{C}_{M A C}\left(\boldsymbol{\nu}, \mathbf{p}_{k}^{*}(\boldsymbol{\nu})\right), \quad k=1,2 .
\end{array}
\end{aligned}
$$


can be reformulated as follows:

$$
\begin{array}{cl}
\max _{\mathbf{r}_{k}, \mathbf{p}_{k}(\boldsymbol{\nu}) \succeq \mathbf{0}, w_{1}(\boldsymbol{\nu})} & \boldsymbol{\mu}^{T} E\left[w_{1}(\boldsymbol{\nu}) \mathbf{r}_{1}-\left(1-w_{1}(\boldsymbol{\nu})\right) \mathbf{r}_{2}\right] \\
\text { s.t. } & E\left[w_{1}(\boldsymbol{\nu}) \mathbf{p}_{1}(\boldsymbol{\nu})+\left(1-w_{1}(\boldsymbol{\nu})\right) \mathbf{p}_{2}(\boldsymbol{\nu})\right] \preceq \mathbf{P}_{a v}, \\
& E\left[w_{1}(\boldsymbol{\nu})\right] \geq 1-\epsilon_{p}, \\
& w_{1}(\boldsymbol{\nu})\left[\mathcal{P}_{p}(g)-\boldsymbol{\beta}^{T} \mathbf{p}_{1}(\boldsymbol{\nu})\right] \geq 0 \\
& \mathbf{r}_{k} \in \mathcal{C}_{M A C}\left(\boldsymbol{\nu}, \mathbf{p}_{k}(\boldsymbol{\nu})\right), \quad k=1,2 \\
& 0 \leq w_{1}(\boldsymbol{\nu}) \leq 1
\end{array}
$$

We will solve the optimization Problem (4.20) by a functional optimization technique similar to [25]. The Lagrangian associated with (4.20) can be written as follows:

$$
\begin{aligned}
& l\left(\boldsymbol{\nu}, \mathbf{p}_{1}(\boldsymbol{\nu}), \mathbf{p}_{2}(\boldsymbol{\nu}), w_{1}(\boldsymbol{\nu}), \lambda, s, \tau(\boldsymbol{\nu})\right) \\
& =w_{1}(\boldsymbol{\nu})\left[\boldsymbol{\mu}^{T} \mathbf{r}_{1}-\boldsymbol{\lambda}^{T} \mathbf{p}_{1}(\boldsymbol{\nu})+s+\tau(\boldsymbol{\nu})\left(\mathcal{P}_{p}(g)-\boldsymbol{\beta}^{T} \mathbf{p}_{1}(\boldsymbol{\nu})\right)\right]+\left(1-w_{1}(\boldsymbol{\nu})\right)\left[\boldsymbol{\mu}^{T} \mathbf{r}_{2}-\boldsymbol{\lambda}^{T} \mathbf{p}_{2}(\boldsymbol{\nu})\right]
\end{aligned}
$$

where $\boldsymbol{\lambda}, s$, and $\tau(\boldsymbol{\nu})$ are the nonnegative Lagrange multipliers corresponding to the constraints (4.20b) to (4.20f), respectively.

As $\mathbf{r}_{k}^{*}$ is a function of $\mathbf{p}_{k}^{*}$, the region $\mathbf{r}_{k}^{*} \in \mathcal{C}_{M A C}\left(\boldsymbol{\nu}, \mathbf{p}_{k}^{*}(\boldsymbol{\nu})\right)$ can be characterized by $2^{M}-1$ constraints for each $k$ and it is not easy to visualize. Instead of applying KKT conditions directly, we can observe that for given $\lambda^{* T}, s^{*}$ and $\tau^{*}(\boldsymbol{\nu})$ with an arbitrary $w_{1}^{*}(\boldsymbol{\nu})$, the optimization problem can be decomposed to two subproblems as follows:

$$
\begin{aligned}
& \text { Subproblem I: } \max _{\mathbf{r}_{1}, \mathbf{p}_{1}(\boldsymbol{\nu})} \boldsymbol{\mu}^{T} \mathbf{r}_{1}-\boldsymbol{\lambda}^{* T} \mathbf{p}_{1}(\boldsymbol{\nu})+s^{*}+\tau^{*}(\boldsymbol{\nu})\left(\mathcal{P}_{p}(g)-\boldsymbol{\beta}^{T} \mathbf{p}_{1}(\boldsymbol{\nu})\right) \\
& \text { s.t. } \quad \mathbf{r}_{1} \in \mathcal{C}_{M A C}\left(\boldsymbol{\nu}, \mathbf{p}_{1}(\boldsymbol{\nu})\right) \text {, } \\
& \tau^{*}(\boldsymbol{\nu})\left(\mathcal{P}_{p}(g)-\boldsymbol{\beta}^{T} \mathbf{p}_{1}(\boldsymbol{\nu})\right)=0 .
\end{aligned}
$$

$$
\begin{aligned}
& \text { Subproblem II: } \max _{\mathbf{r}_{2}, \mathbf{p}_{2}(\boldsymbol{\nu})} \boldsymbol{\mu}^{T} \mathbf{r}_{2}-\boldsymbol{\lambda}^{* T} \mathbf{p}_{2}(\boldsymbol{\nu}) \\
& \text { s.t. } \quad \mathbf{r}_{2} \in \mathcal{C}_{M A C}\left(\boldsymbol{\nu}, \mathbf{p}_{2}(\boldsymbol{\nu})\right) \text {. }
\end{aligned}
$$


Indeed, the objective of (4.22) is equivalent to maximizing $\boldsymbol{\mu}^{T} \mathbf{r}_{1}-\left(\boldsymbol{\lambda}^{*}+\tau^{*}(\boldsymbol{\nu}) \boldsymbol{\beta}\right)^{T} \mathbf{p}_{1}(\boldsymbol{\nu})$ as $s^{*}$ is a long-term Lagrange multiplier and $\mathcal{P}_{p}(g)$ is independent on $\left(\mathbf{r}_{1}, \mathbf{p}_{1}\right)$. Furthermore, observe that Subproblem I corresponds to capacity region with instantaneous interference power constraint with the threshold $\mathcal{P}_{p}(g)$. When $\tau^{*}(\boldsymbol{\nu})=0$, the solution to Subproblem I and Subproblem II become similar and the solution was previously shown in [14]. So, now we will study the case that $\tau^{*}(\boldsymbol{\nu})>0$ only. In other words, $\tau^{*}(\boldsymbol{\nu})>0$ is selected such that $\mathcal{P}_{p}(g)=\boldsymbol{\beta}^{T} \mathbf{p}_{1}(\boldsymbol{\nu})$.

Hence, define $\boldsymbol{\eta}=\left[\eta_{1}, \ldots, \eta_{M}\right]$ where $\eta_{i}=\lambda_{i}^{*}+\tau^{*}(\boldsymbol{\nu}) \beta_{i}$. So for a given $\boldsymbol{\lambda}^{*}$ and $\tau^{*}(\boldsymbol{\nu}),(4.22)$ can be rewritten as

$$
\begin{array}{lll}
\text { Subproblem I: } & \max _{\mathbf{r}_{1}, \mathbf{p}_{1}(\boldsymbol{\nu})} & \boldsymbol{\mu}^{T} \mathbf{r}_{1}-\boldsymbol{\eta}^{T} \mathbf{p}_{1}(\boldsymbol{\nu}) \\
& \text { s.t. } & \mathbf{r}_{1} \in \mathcal{C}_{M A C}\left(\boldsymbol{\nu}, \mathbf{p}_{1}(\boldsymbol{\nu})\right)
\end{array}
$$

To solve this, we re-state Theorem 3.14 in [14] as follows:

\section{Theorem 4.2.2.}

$$
\max _{\mathbf{x}, \mathbf{y}} \boldsymbol{\mu}^{T} \cdot \mathbf{x}-\Lambda^{T} \cdot \mathbf{y} \quad \text { s.t. } \mathbf{x}(S) \leq \mathscr{G}(\mathbf{y}(S)) \quad \forall S \subset \mathcal{E}
$$

where $\mathscr{G}$ is a monotonically increasing concave function. Define the marginal utility functions

$$
\begin{aligned}
u_{i}(z) & \equiv \mu_{i} \mathscr{G}^{\prime}(z)-\Lambda_{i} \quad i=1, \ldots, M \\
u^{*}(z) & \equiv\left[\max _{i} u_{i}(z)\right]^{+} .
\end{aligned}
$$

Then, the solution to (4.25) is given by $\int_{z=0}^{\infty} u^{*}(z) d z$ and an optimizing point $\left(\mathbf{x}^{*}, \mathbf{y}^{*}\right)$ to achieve this can be found by an appropriate greedy algorithm.

By Theorem 4.2.2, we define $\mathscr{G}(z) \equiv \log (1+z)$ and $\mathbf{q}_{1}=\left[\bar{h}_{1} p_{1, i}, \ldots, \bar{h}_{M} p_{1 M}\right]^{T}$ which can be interpreted as the vector of the received SNR from each ST to the SBS. Hence, (4.24) becomes

$$
\max _{\mathbf{r}_{1}, \mathbf{q}} \sum_{i=1}^{M} \mu_{i} r_{1, i}-\sum_{i=1}^{M} \frac{\eta_{i}}{h_{i}} q_{1, i} \quad \text { s.t. } \mathbf{r}_{1}(S) \leq \log \left(1+\mathbf{q}_{1}(S)\right) \quad \forall S \subset \mathcal{E}
$$

Define the marginal utility functions $u_{i}(z)=\frac{\mu_{i}}{1+z}-\frac{\eta_{i}}{h_{i}}$ in which $u_{i}(z) \cdot d z$ can be 
interpreted as the difference between rate revenue $\left(\frac{\mu_{i}}{1+z} \cdot d z\right)$ and power cost $\left(\frac{\eta_{i}}{h_{i}} \cdot d z\right)$ at the interference level $1+z$. Also, let $u^{*}(z)=\left[\max _{i} u_{i}(z)\right]^{+}$be the the highest marginal utility function. The user with the highest marginal utility function will be decoded at the interference level $1+z$. Further notice that when $\tau^{*}(\boldsymbol{\nu})>0$, $\eta_{i}=\lambda_{i}^{*}+\tau^{*}(\boldsymbol{\nu}) \beta_{i}>\lambda_{i}^{*}$ implying that the instantaneous interference power constraint causes an increase in the power price that each SU has to pay and the additional cost also depends on value of $\beta_{i}$, i.e. whether the interference channel gain from $\mathrm{SU}_{i}$ to $\mathrm{PU}$ is strong or weak. With a similar technique previously used in [14], the problem (4.27) is equivalent to.

$$
\max _{\mathbf{q}}\left[\mu_{\pi(1)} \log \left(1+q_{\pi(1)}\right)-\frac{\eta_{\pi(1)}}{h_{\pi(1)}} q_{\pi(1)}\right]+\sum_{i=2}^{M}\left[\mu_{\pi(i)} \log \left(1+\frac{q_{\pi(i)}}{1+\sum_{k=1}^{i-1} q_{\pi(k)}}\right)-\frac{\eta_{\pi(i)}}{h_{\pi(i)}} q_{\pi(i)}\right]
$$

where $\pi($.$) be the permutation representing a decreasing order of the vector \boldsymbol{\mu}$.

Therefore, the optimal solution can be achieved by successive decoding for all cases. $\pi($.$) represents the decoding order function. The signal from \mathrm{SU}_{\pi(M)}$ is decoded first, treating the signal from $\mathrm{SU}_{\pi(1)}$ to $\mathrm{SU}_{\pi(M-1)}$ as noise. Then, the decoded signal from $\mathrm{SU}_{\pi(M)}$ is subtracted from the total received power and $\mathrm{SU}_{\pi(M-1)}$ 's message is decoded while treating the signal from $\mathrm{SU}_{\pi(1)}$ to $\mathrm{SU}_{\pi(M-2)}$ as noise. $\mathrm{SU}_{\pi(1)}$ is decoded at the end. Consequently, $\pi($.$) also reflects the priority among the users$ since, for instance, $\mathrm{SU}_{\pi(M)}$ has to deal with not merely the background noise but also the interference from the other users. Here $z$ corresponds to the total interfering power received from other users while $\mathrm{SU}_{\pi(i)}$ is decoded, making the total interference equal $1+z_{i}$. In other words, the solution (4.28) can be interpreted as the choice of users that will transmit at the noise level $1+z$ for $z \in[0, \infty)$.

Note that in order to find the maximum utility function for a given channel state $\boldsymbol{\nu}$, there are four possible cases that need to be considered. The first case is when $\mu_{i} \neq \mu_{j}$ and $\frac{\eta_{i}}{h_{i}} \neq \frac{\eta_{j}}{h_{j}}$. In this case, the two curves will intersect at most once as $g^{\prime}(z)$ is monotone. Thus, only one user with the highest marginal utility function will transmit at that interference level $1+z$. The second case is when $\mu_{i}=\mu_{j}$ and $\frac{\eta_{i}}{h_{i}} \neq \frac{\eta_{j}}{h_{j}}$. Without loss of generality, suppose that $\frac{\eta_{i}}{h_{i}}>\frac{\eta_{j}}{h_{j}}$, then there 
is no power allocated to the $i$-th user as $u_{i}(z)<u_{j}(z)$ for all $z$. The third case is when $\mu_{i} \neq \mu_{j}$ and $\frac{\eta_{i}}{h_{i}}=\frac{\eta_{j}}{h_{j}}$. Suppose that $\mu_{i}<\mu_{j}$. Then the $i$-th user will not transmit as $u_{i}(z)<u_{j}(z)$ for all $z$. The last case is when $\mu_{i}=\mu_{j}$ and $\frac{\eta_{i}}{h_{i}}=\frac{\eta_{j}}{h_{j}}$, making $u_{i}(z)=u_{j}(z)$ for all $z$. Thus, if $u_{i}(z)=u_{j}(z)=u^{*}(z)$, the $i$-th and the $j$-th secondary transmitters are allowed to transmit at that interference level $1+z$.

Lemma 4.2.4. There are at most two users that satisfy $\frac{\eta_{i}}{h_{i}}=\frac{\eta_{j}}{h_{j}}$ for $i \neq j$.

Proof. The proof is similar to the proof of Lemma 4.2 .2 by showing that there exists at most two SUs that satisfy $\frac{\lambda_{i}^{*}+\tau^{*} \beta_{i}}{\bar{h}_{i}}=\frac{\lambda_{j}^{*}+\tau^{*} \beta_{j}}{\bar{h}_{j}}$ for $i \neq j$.

By Lemma 4.2.4 and considering the four cases for finding the maximum utility function, we can conclude that there are at most two users allowed to transmit at the same interference level $1+z$ and this happens only when $u^{*}(z)=u_{i}(z)=u_{j}(z)$. Now we will investigate the rate and power obtained by each user in the specific channel state $\boldsymbol{\nu}$.

Case I: One user is allowed to transmit at the interference level $1+z$

It means the curves $u_{i}(z)$ and $u_{j}(z)$ intersect at most once. For channel state $\boldsymbol{\nu}$, the $i$-th user is therefore allocated the power with $p_{1, i}^{*}(\boldsymbol{\nu})=\frac{1}{h_{i}} \int_{\mathcal{A}_{i}} d z$ and the rate $r_{1, i}^{*}=\int_{\mathcal{A}_{i}} \frac{1}{1+z} d z$ where $\mathcal{A}_{i} \equiv\left\{z \in[0, \infty): u_{i}(z)=u^{*}(z)\right.$ and $\left.u_{i}(z)>0\right\}$ such that $\mathcal{P}_{p}(g)=\boldsymbol{\beta}^{T} \mathbf{p}_{1}^{*}$.

Case II: Two users are allowed to transmit at the interference level $1+z$

Suppose $\mathrm{SU}_{v}$ and $\mathrm{SU}_{b}$ satisfy the conditions stated above. Then $\mu_{v}=\mu_{b}=\mu_{\pi(K)}$. Without loss of generality, we can assume that, after permutation $\pi(),. v=\pi(K)$ and $b=\pi(K+1)$. Further, $\frac{\eta_{\pi(K)}}{h_{\pi(K)}}=\frac{\eta_{\pi(K+1)}}{h_{\pi(K+1)}}$. From the problem (4.28), the term 
related to $\mathrm{SU}_{v}$ and $\mathrm{SU}_{b}$ becomes

$$
\begin{aligned}
& {\left[\mu_{\pi(K)} \log \left(1+\frac{q_{\pi(K)}}{1+\sum_{i=1}^{K-1} q_{\pi(i)}}\right)-\frac{\eta_{\pi(K)}}{h_{\pi(K)}} q_{\pi(K)}\right]} \\
& +\left[\mu_{\pi(K+1)} \log \left(1+\frac{q_{\pi(K+1)}}{1+\sum_{k=1}^{K} q_{\pi(i)}}\right)-\frac{\eta_{\pi(K+1)}}{h_{\pi(K+1)}} q_{\pi(K+1)}\right] \\
& =\mu_{\pi(K)}\left[\log \left(1+\frac{q_{\pi(K)}+q_{\pi(K+1)}}{1+\sum_{i=1}^{K-1} q_{\pi(i)}}\right)\right]-\left[\frac{\eta_{\pi(K)}}{h_{\pi(K)}} q_{\pi(K)}+\frac{\eta_{\pi(K+1)}}{h_{\pi(K+1)}} q_{\pi(K+1)}\right] \\
& =\mu_{\pi(K)}\left[\log \left(1+\frac{q_{\pi(K)}+q_{\pi(K+1)}}{1+\sum_{i=1}^{K-1} q_{\pi(i)}}\right)\right]-\frac{\eta_{\pi(K)}}{h_{\pi(K)}}\left[q_{\pi(K)}+q_{\pi(K+1)}\right] \\
& \stackrel{(a)}{=} \mu_{\pi(K)}\left[\log \left(1+\frac{\tilde{q}_{\pi(K), \pi(K+1)}}{1+\sum_{i=1}^{K-1} q_{\pi(i)}}\right)\right]-\frac{\eta_{\pi(K)}}{\bar{h}_{\pi(K)}}\left[\tilde{q}_{\pi(K), \pi(K+1)}\right] \\
& \stackrel{(b)}{=}\left[\mu_{\tilde{\pi}(K)} \log \left(1+\frac{q_{\tilde{\pi}(K)}}{1+\sum_{i=1}^{K-1} q_{\tilde{\pi}}}\right)-\frac{\eta_{\tilde{\pi}(K)}}{h_{\tilde{\pi}(K)}} q_{\tilde{\pi}(K)}\right] \\
& +\left[\mu_{\tilde{\pi}(K+1)} \log \left(1+\frac{q_{\tilde{\pi}(K+1)}}{1+\sum_{k=1}^{K} q_{\tilde{\pi}(i)}}\right)-\frac{\eta_{\tilde{\pi}(K+1)}}{h_{\tilde{\pi}(K+1)}} q_{\tilde{\pi}(K+1)}\right]
\end{aligned}
$$

where $\tilde{q}_{\pi(K), \pi(K+1)}=q_{\pi(K)}+q_{\pi(K+1)}$ and $\tilde{\pi}($.$) is another permutation such that$ $\tilde{\pi}(K)=\pi(K+1), \tilde{\pi}(K+1)=\pi(K)$ and $\tilde{\pi}(i)=\pi(i)$ for all $i \neq K, K+1$.

Remark 4.2.4. The steps $(a),(b)$ in (4.29) reveal that the solution is still successive decoding; however, the two users are treated as if they were combined as a single user. So in this case, the allocated power $\mathbf{p}_{1}^{*}$ must satisfy the set of equations in (4.30).

$$
\begin{array}{lll}
p_{1, i}^{*} & =\frac{1}{h_{i}} \int_{\mathcal{A}_{i}} d z \quad \text { for } i \neq \pi(K), \pi(K+1) \\
\bar{h}_{\pi(K)} p_{1, \pi(K)}^{*}+\bar{h}_{\pi(K+1)} p_{1, \pi(K+1)}^{*} & =\int_{\mathcal{A}^{\prime}} d z=\tilde{q}_{\pi(K), \pi(K+1)} \\
\mathcal{P}_{p}(g) & =\boldsymbol{\beta}^{T} \mathbf{p}_{1}^{*}
\end{array}
$$

where $\mathcal{A}^{\prime} \equiv\left\{z \in[0, \infty): u_{\pi(K)}(z)=u_{\pi(K+1)}(z)=u^{*}(z)\right.$ and $\left.u_{\pi(K)}(z)>0\right\}$. Thus, 
the sum rate from the users $\mathrm{SU}_{\pi(K)}$ and $\mathrm{SU}_{\pi(K+1)}$ after decoding becomes $r_{1, \pi(K)}^{*}+$ $r_{1, \pi(K+1)}^{*}=\int_{\mathcal{A}^{\prime}} \frac{1}{1+z} d z$ and the rate $r_{1, i}^{*}=\int_{\mathcal{A}_{i}} \frac{1}{1+z} d z$ for other secondary users. The condition in (4.30) will be used in Section 4.2 .3 when we discuss the relationship between the ergodic sum capacity maximizing problem in (4.6) and the ergodic capacity region problem in (4.20).

Remark 4.2.5. The result of $(b)$ in (4.29) reveals that in the decoding process, the SBS can use another permutation $\tilde{\pi}($.$) such that \tilde{\pi}(K)=\pi(K+1), \tilde{\pi}(K+1)=\pi(K)$ and $\tilde{\pi}(i)=\pi(i)$ for all $i \neq K, K+1$ while obtaining the same result as using permutation $\pi($.$) . In other words, although \mathrm{SU}_{v}$ and $\mathrm{SU}_{b}$ transmit simultaneously at the interference level $1+z$, switching the decoding order between $v$ and $b$ at the SBS does not affect the achievable rate.

By using the KKT conditions and the fact that continuous fading channels are assumed, the solution for $w_{1}^{*}(\boldsymbol{\nu})$ becomes

$$
w_{1}^{*}(\boldsymbol{\nu})=\left\{\begin{array}{cc}
0, & \boldsymbol{\mu}^{T} \mathbf{r}_{1}-\boldsymbol{\lambda}^{* T} \mathbf{p}_{1}^{*}(\boldsymbol{\nu})+s^{*}<\boldsymbol{\mu}^{T} \mathbf{r}_{2}-\boldsymbol{\lambda}^{* T} \mathbf{p}_{2}^{*}(\boldsymbol{\nu}) \\
1, & \boldsymbol{\mu}^{T} \mathbf{r}_{1}-\boldsymbol{\lambda}^{* T} \mathbf{p}_{1}^{*}(\boldsymbol{\nu})+s^{*}>\boldsymbol{\mu}^{T} \mathbf{r}_{2}-\boldsymbol{\lambda}^{* T} \mathbf{p}_{2}^{*}(\boldsymbol{\nu})
\end{array}\right.
$$

Note that $\boldsymbol{\mu}^{T} \mathbf{r}_{1}-\boldsymbol{\lambda}^{* T} \mathbf{p}_{1}^{*}(\boldsymbol{\nu})+s^{*}$ and $\boldsymbol{\mu}^{T} \mathbf{r}_{2}-\boldsymbol{\lambda}^{* T} \mathbf{p}_{2}^{*}(\boldsymbol{\nu})$ serve as the benefit of using strategy $\mathbf{p}_{1}^{*}(\boldsymbol{\nu})$ and $\mathbf{p}_{2}^{*}(\boldsymbol{\nu})$, respectively. The secondary network will use the strategy that returns the highest profit.

\subsubsection{Discussion}

In this part we clarify the relationship between the optimal power policies in Sections 4.2.1 and 4.2.2. For the ergodic sum rate, it implies that $\mu_{1}=\ldots=\mu_{M}=\mu$. Without loss of generality, we can assign $\mu=1$. For power policy $p_{2, i}(\boldsymbol{\nu})$ and for a specific $\boldsymbol{\nu}$, only the $i$-th user is allowed to transmit when $i=\arg \min _{m \in \mathcal{E}} \frac{\lambda_{i}}{\bar{h}_{i}}$ since $\left[u_{i}(z)\right]^{+}=\left[\frac{\mu_{i}}{1+z}-\frac{\lambda_{i}}{h_{i}}\right]^{+}=u^{*}(z)$ for all $z$. We then have

$$
p_{2, i}^{*}(\boldsymbol{\nu})= \begin{cases}\frac{1}{h_{i}} \int_{z=0}^{\infty} \mathbf{1}\left\{\frac{1}{1+z}-\frac{\lambda_{i}}{h_{i}}>0\right\} d z=\left[\frac{1}{\lambda_{i}}-\frac{1}{h_{i}}\right]^{+} & , i=\arg \max _{m \in \mathcal{E}} \frac{\bar{h}_{m}}{\lambda_{m}^{*}} \\ 0 & , \text { else }\end{cases}
$$


The solution is exactly the same as shown in Section 4.2.1, i.e. $\mathbf{p}_{2}(\boldsymbol{\nu})=\mathbf{p}_{W F}^{*}(\boldsymbol{\nu})$.

In the same manner, $p_{1, i}^{*}(\boldsymbol{\nu})$ can be investigated. When $\tau^{*}(\boldsymbol{\nu})=0$, the solution is the same as (4.32) and at most one user transmits. When $\tau^{*}(\boldsymbol{\nu})>0$, it means $\boldsymbol{\beta}^{T} \mathbf{p}_{1}^{*}(\boldsymbol{\nu})=\mathcal{P}_{p}(g)$. Note that in this case, we have two subcases

\section{One user case}

As $\mu_{i}=1$ for all $i$, it means that there exists only the $i$-th user with the $\operatorname{minimum} \frac{\lambda_{i}^{*}+\tau^{*}(\boldsymbol{\nu}) \beta_{i}}{\bar{h}_{i}}$

$$
p_{1, i}^{*}(\boldsymbol{\nu})= \begin{cases}\frac{1}{h_{i}} \int_{z=0}^{\infty} 1\left\{\frac{1}{1+z}-\frac{\lambda_{i}^{*}+\tau^{*}(\boldsymbol{\nu}) \beta_{i}}{\bar{h}_{i}}>0\right\} d z & \stackrel{\stackrel{(a)}{=} \frac{\mathcal{P}_{p}(p)}{\beta_{i}}}{ } \\ 0 & ,\left(i=\arg \max _{m \in \mathcal{E}} \frac{\bar{h}_{m}}{\lambda_{m}^{*}+\tau^{*}(\boldsymbol{\nu}) \beta_{m}}\right) \\ & , \text { otherwise }\end{cases}
$$

Note that $(a)$ follows from $\boldsymbol{\beta}^{T} \mathbf{p}_{1}^{*}(\boldsymbol{\nu})=\mathcal{P}_{p}(g)$. So, the solution reflects the same result as in (4.12).

\section{Two users case}

In this case there are two users: $\mathrm{SU}_{v}$ and $\mathrm{SU}_{b}$ with the minimum $\frac{\lambda_{v}^{*}+\tau^{*}(\boldsymbol{\nu}) \beta_{v}}{\bar{h}_{v}}=$ $\frac{\lambda_{b}^{*}+\tau^{*}(\boldsymbol{\nu}) \beta_{b}}{\bar{h}_{b}}$, making $u^{*}(z)=\left[u_{v}(z)\right]^{+}=\left[u_{b}(z)\right]^{+}$for all $z$. Thus, the achievable sum rate by $\mathbf{p}_{1}^{*}(\boldsymbol{\nu})$ can be described as follows:

$$
\begin{aligned}
r_{s}^{*}=r_{1, v}^{*}(\boldsymbol{\nu})+r_{1, b}^{*}(\boldsymbol{\nu}) & =\int_{z=0}^{\infty} \frac{1}{1+z} \mathbf{1}\left\{\frac{1}{1+z}-\frac{\lambda_{i}^{*}+\tau^{*}(\boldsymbol{\nu}) \beta_{i}}{\bar{h}_{i}}>0\right\} d z \\
& =\log \left(\frac{\bar{h}_{v}}{\lambda_{v}^{*}+\tau^{*}(\boldsymbol{\nu}) \beta_{v}}\right)
\end{aligned}
$$

By the definition of sum rate, it leads to $1+\sum_{i \in E} p_{1, i}^{*}(\boldsymbol{\nu}) \bar{h}_{i}=\frac{\bar{h}_{v}}{\lambda_{v}^{*}+\tau^{*}(\boldsymbol{\nu}) \beta_{v}}=$ $\frac{\bar{h}_{b}}{\lambda_{b}^{*}+\tau^{*}(\boldsymbol{\nu}) \beta_{b}}$. Also, $\boldsymbol{\beta}^{T} \mathbf{p}_{1}^{*}(\boldsymbol{\nu})=\mathcal{P}_{p}(g)$. Thus, the results are the same as the condition in (4.30) which $\tilde{q}_{\pi(K), \pi(K+1)}=\frac{\bar{h}_{v}}{\lambda_{v}^{*}+\tau^{*}(\boldsymbol{\nu}) \beta_{v}}-1$. After rearranging, the same result expressed in (4.13) and (4.14) can also be obtained.

The optimal values $w_{1}^{*}(\boldsymbol{\nu})$ in the derivations in Section 4.2.1 and 4.2.2 are obviously the same. 


\subsection{Outage capacity region}

In this section, we will focus on outage capacity for the $M$-user secondary uplink network under ATPC and POC. There are two types of outage capacity definitions that have been previously studied, including common outage capacity and individual outage capacity in [22] for non-cognitive environment and in [53] for cognitive environment under an instantaneous interference constraint at the primary network. Therefore, we start Section 4.3 with the descriptions of those two outage capacities. Prior to solving the optimal power control policies, we will show that the outage capacity region (both common and individual) is implicitly obtained by deriving the outage probability region for a given rate vector. Therefore, we will explicitly derive the optimal power control policies to achieve common outage probability region in Section 4.3.2 and individual outage probability region in Section 4.3.3, severally.

\subsubsection{Definition of common outage capacity and individual outage capacity}

In both non-cognitive and cognitive MAC, there are two outage scenarios in that have been studied, i.e. common outage capacity and individual outage capacity. The definition of those two outage capacities are as follows:

Definition 4.3.1. A rate vector is said to be in the common outage capacity region $\mathcal{C}_{o}\left(\mathbf{P}_{a v}, \epsilon_{p}, \epsilon_{s}\right)$ if and only if there exists a random power vector $\mathbf{P}(\boldsymbol{\nu})$ that satisfies (4.2) and (4.3), and allows the rate vector to be achieved with a probability of at least $1-\epsilon_{s}$, i.e. $\operatorname{Pr}\left\{\mathbf{r}_{o} \in \mathcal{C}_{M A C}(\boldsymbol{\nu}, \mathbf{P}(\boldsymbol{\nu}))\right\} \geq 1-\epsilon_{s}$. The common outage capacity region is then expressed as

$$
\mathcal{C}_{o}\left(\mathbf{P}_{a v}, \epsilon_{p}, \epsilon_{s}\right) \triangleq \bigcup_{\mathbf{P}(\boldsymbol{\nu}) \in \mathcal{F}}\left\{\mathbf{r}_{o}: \operatorname{Pr}\left\{\mathbf{r} \in \mathcal{C}_{M A C}(\boldsymbol{\nu}, \mathbf{P}(\boldsymbol{\nu}))\right\} \geq 1-\epsilon_{s}\right\}
$$

where $\mathcal{F} \triangleq\{\mathbf{P}(\boldsymbol{\nu}):$ (4.2), (4.3) $\}$

Definition 4.3.1 reveals that the common outage capacity region consists of all rate vectors that can be maintained with a common outage probability no larger 
than $\epsilon_{s}$ under ATPC and POC. It is also observable that the non-outage event or so called usage event is declared simultaneously to all users.

Definition 4.3.2. A rate vector $\mathbf{r}_{o}=\left[r_{o, 1}, \ldots, r_{o, M}\right]^{T}$ is said to be in the individual outage capacity region $\mathcal{C}_{o}\left(\mathbf{P}_{a v}, \epsilon_{p}, \boldsymbol{\epsilon}_{s}\right)$ if and only if there exists a random power vector $\mathbf{P}(\boldsymbol{\nu})$ that satisfies (4.2) and (4.3), and a corresponding vector of rate allocation function $\mathbf{r}(\boldsymbol{\nu}, \mathbf{P}(\boldsymbol{\nu})) \in \mathcal{C}_{M A C}(\boldsymbol{\nu}, \mathbf{P}(\boldsymbol{\nu}))$ for each fading state $\boldsymbol{\nu}$ such that $\operatorname{Pr}\left\{r_{i}(\boldsymbol{\nu}, \mathbf{P}(\boldsymbol{\nu})) \geq r_{o, i}\right\} \geq 1-\epsilon_{s, i}$. The individual outage capacity region is defined as

$$
\mathcal{C}_{o}\left(\mathbf{P}_{a v}, \epsilon_{p}, \boldsymbol{\epsilon}_{s}\right) \triangleq \bigcup_{\mathbf{P}(\boldsymbol{\nu}) \in \mathcal{F}}\left\{\mathbf{r}_{o}: \operatorname{Pr}\left\{r_{i}(\boldsymbol{\nu}, \mathbf{P}(\boldsymbol{\nu})) \geq r_{o, i}\right\} \geq 1-\epsilon_{s, i}, \forall i\right\}
$$

where $\mathcal{F} \triangleq\{\mathbf{P}(\boldsymbol{\nu}):$ (4.2), (4.3) $\}$.

In other words, Definition 4.3.2 implies that the individual outage capacity region consists of all rate vector that can be maintained with an outage probability vector.

Remark 4.3.1. The simple explanation of the difference of those two outage capacity notion is how we define outage event. In the common outage capacity, the non-outage event happens when target data rates $\mathbf{r}_{o}$ of all STs are satisfied. In the individual outage capacity, the non-outage outage events are treated individually in each ST. So for some channel realization $\boldsymbol{\nu}$, the secondary network is allowed to neglect some STs and satisfy the service requirements of the remaining STs. This provides the secondary network more options to transmit, thus making the individual outage capacity problem more lenient than that of common outage capacity.

\subsubsection{Common outage capacity}

This section will consider how to achieve each boundary points of the common outage capacity region as defined in Definition 4.3.1. Define weighting functions $\psi_{k}(\boldsymbol{\nu}), k=1,2,3,4$ and the corresponding power control for four possible situations 
that can happen.

$$
\begin{array}{cl}
\psi_{1}(\boldsymbol{\nu})= & \operatorname{Pr}\left\{r_{p}(\boldsymbol{\nu}, \mathbf{P}(\boldsymbol{\nu})) \geq r_{p}^{0}, \mathbf{r}_{o} \in \mathcal{C}_{M A C}(\boldsymbol{\nu}, \mathbf{P}(\boldsymbol{\nu})) \mid \boldsymbol{\nu}\right\} \\
\psi_{2}(\boldsymbol{\nu})= & \operatorname{Pr}\left\{r_{p}(\boldsymbol{\nu}, \mathbf{P}(\boldsymbol{\nu}))<r_{p}^{0}, \mathbf{r}_{o} \in \mathcal{C}_{M A C}(\boldsymbol{\nu}, \mathbf{P}(\boldsymbol{\nu})) \mid \boldsymbol{\nu}\right\} \\
\psi_{3}(\boldsymbol{\nu})= & \operatorname{Pr}\left\{r_{p}(\boldsymbol{\nu}, \mathbf{P}(\boldsymbol{\nu})) \geq r_{p}^{0}, \mathbf{r}_{o} \notin \mathcal{C}_{M A C}(\boldsymbol{\nu}, \mathbf{P}(\boldsymbol{\nu})) \mid \boldsymbol{\nu}\right\} \\
\psi_{4}(\boldsymbol{\nu})= & \operatorname{Pr}\left\{r_{p}(\boldsymbol{\nu}, \mathbf{P}(\boldsymbol{\nu}))<r_{p}^{0}, \mathbf{r}_{o} \notin \mathcal{C}_{M A C}(\boldsymbol{\nu}, \mathbf{P}(\boldsymbol{\nu})) \mid \boldsymbol{\nu}\right\} \\
\mathbf{p}_{1}(\boldsymbol{\nu})= & E\left[\mathbf{P}(\boldsymbol{\nu}) \mid r_{p}(\boldsymbol{\nu}, \mathbf{p}(\boldsymbol{\nu})) \geq r_{p}^{0}, \mathbf{r}_{o} \in \mathcal{C}_{M A C}(\boldsymbol{\nu}, \mathbf{p}(\boldsymbol{\nu})), \boldsymbol{\nu}\right] \\
\mathbf{p}_{2}(\boldsymbol{\nu})= & E\left[\mathbf{P}(\boldsymbol{\nu}) \mid r_{p}(\boldsymbol{\nu}, \mathbf{p}(\boldsymbol{\nu}))<r_{p}^{0}, \mathbf{r}_{o} \in \mathcal{C}_{M A C}(\boldsymbol{\nu}, \mathbf{p}(\boldsymbol{\nu})), \boldsymbol{\nu}\right] \\
\mathbf{p}_{3}(\boldsymbol{\nu})= & E\left[\mathbf{P}(\boldsymbol{\nu}) \mid r_{p}(\boldsymbol{\nu}, \mathbf{p}(\boldsymbol{\nu})) \geq r_{p}^{0}, \mathbf{r}_{o} \notin \mathcal{C}_{M A C}(\boldsymbol{\nu}, \mathbf{p}(\boldsymbol{\nu})), \boldsymbol{\nu}\right] \\
\mathbf{p}_{4}(\boldsymbol{\nu})= & E\left[\mathbf{P}(\boldsymbol{\nu}) \mid r_{p}(\boldsymbol{\nu}, \mathbf{p}(\boldsymbol{\nu}))<r_{p}^{0}, \mathbf{r}_{o} \notin \mathcal{C}_{M A C}(\boldsymbol{\nu}, \mathbf{p}(\boldsymbol{\nu})), \boldsymbol{\nu}\right]
\end{array}
$$

Lemma 4.3.1. $\mathcal{C}_{o}\left(\mathbf{P}_{a v}, \epsilon_{p}, \epsilon_{s}\right)$ is achieved by a power policy $\mathbf{P}(\boldsymbol{\nu})$ of the form $\mathbf{P}^{*}(\boldsymbol{\nu})=\sum_{k=1}^{4} X_{\psi_{k}(\boldsymbol{\nu})}(\boldsymbol{\nu}) \mathbf{p}_{k}(\boldsymbol{\nu})$ where

- (1) $E\left[\psi_{1}(\boldsymbol{\nu})+\psi_{2}(\boldsymbol{\nu})\right] \geq 1-\epsilon_{s}$,

(2) $E\left[\sum_{k=1}^{4} \psi_{k}(\boldsymbol{\nu}) \mathbf{p}_{k}(\boldsymbol{\nu})\right] \preceq \mathbf{P}_{a v}$,

(3) $E\left[\psi_{1}(\boldsymbol{\nu})+\psi_{3}(\boldsymbol{\nu})\right] \geq 1-\epsilon_{p}$,

(4) $\psi_{k}(\boldsymbol{\nu})\left[\mathcal{P}_{p}(g)-\boldsymbol{\beta}^{T} \mathbf{p}_{k}(\boldsymbol{\nu})\right] \geq 0, \quad k=1,3$

(5) $1-\sum_{k=1}^{4} \psi_{k}(\boldsymbol{\nu})=0$

(6) $\mathbf{p}_{k}(\boldsymbol{\nu}) \succeq \mathbf{0}, \quad k=1,2,3,4$,

(7) $\psi_{k}(\boldsymbol{\nu}) \geq 0, \quad k=1,2,3,4$,

(8) $\mathbf{r}_{o} \in \mathcal{C}_{M A C}\left(\boldsymbol{\nu}, \mathbf{p}_{k}(\boldsymbol{\nu})\right), \quad k=1,2$

Proof. The proof of Lemma 4.3.1 is included in Appendix C.5.

Lemma 4.3.1 simply describes the characteristics of the optimal solution $\mathbf{P}^{*}(\boldsymbol{\nu})$ which is randomized amongst the four deterministic power policies $\mathbf{p}_{k}(\boldsymbol{\nu})$. Thus, we have to find the optimal solutions for $\mathbf{p}_{k}(\boldsymbol{\nu})$ and $\psi_{k}(\boldsymbol{\nu})$. However, the common outage capacity region and the corresponding solutions of $\mathbf{p}_{k}(\boldsymbol{\nu})$ and $\psi_{k}(\boldsymbol{\nu})$ are difficult to obtain explicitly by directly characterizing its boundary points. Instead, we can show that the common outage capacity region can be implicitly obtained by deriving the common outage probability region for each given rate vector. To do so, Definitions 4.3.3 to 4.3.5 and Proposition 4.3.1 below are required.

Definition 4.3.3. The outage probability $\epsilon_{s}$ is in the common outage probability set $\mathcal{O}_{C}\left(\mathbf{P}_{a v}, \epsilon_{p}, \mathbf{r}_{o}\right)$ if and only if the rate vector $\mathbf{r}_{o} \in \mathcal{C}_{o}\left(\mathbf{P}_{a v}, \epsilon_{p}, \epsilon_{s}\right)$. 
Definition 4.3.4. The usage probability $\bar{\epsilon}_{s}$ is in the common usage probability set $\overline{\mathcal{O}}_{C}\left(\mathbf{P}_{a v}, \epsilon_{p}, \mathbf{r}_{o}\right)$ if and only if the rate vector $\mathbf{r}_{o} \in \mathcal{C}_{o}\left(\mathbf{P}_{a v}, \epsilon_{p}, 1-\bar{\epsilon}_{s}\right)$.

Definition 4.3.5. The minimum common outage probability $\operatorname{Pr}_{\min }\left(\mathbf{P}_{a v}, \epsilon_{p}, \mathbf{r}_{o}\right)$ is the smallest probability in the set $\mathcal{O}_{C}\left(\mathbf{P}_{a v}, \epsilon_{p}, \mathbf{r}_{o}\right)$.

Proposition 4.3.1. The common usage probability set $\overline{\mathcal{O}}_{C}\left(\mathbf{P}_{a v}, \epsilon_{p}, \mathbf{r}_{o}\right)$ is equivalently given by

$$
\overline{\mathcal{O}}_{C}\left(\mathbf{P}_{a v}, \epsilon_{p}, \mathbf{r}_{o}\right)=\bigcup_{\left(\psi_{k}(\boldsymbol{\nu}), p_{k}(\boldsymbol{\nu})\right) \in \mathcal{F}} E\left[\psi_{1}(\boldsymbol{\nu})+\psi_{2}(\boldsymbol{\nu})\right]
$$

where $\mathcal{F} \triangleq\left\{\left(\psi_{k}(\boldsymbol{\nu}), p_{k}(\boldsymbol{\nu})\right)\right.$ : the constraints (2) to (8) in Lemma. 4.3.1 $\}$

Proof. The common usage probability $\bar{\epsilon}_{s}$ is in the common usage probability set if and only if the rate vector $\mathbf{r}_{o} \in \mathcal{C}_{o}\left(\mathbf{P}_{a v}, \epsilon_{p}, 1-\bar{\epsilon}_{s}\right)$, which is true if and only if the constraints in Lemma. 4.3.1 are satisfied. Then, we have

$$
\overline{\mathcal{O}}_{C}\left(\mathbf{P}_{a v}, \epsilon_{p}, \mathbf{r}_{o}\right)=\bigcup_{\left(\psi_{k}(\boldsymbol{\nu}), p_{k}(\boldsymbol{\nu})\right) \in \mathcal{F}}\left[0, E\left[\psi_{1}(\boldsymbol{\nu})+\psi_{2}(\boldsymbol{\nu})\right]\right]
$$

However, notice that if $\mathbf{P}(\boldsymbol{\nu})$ exists and $E\left[\psi_{1}(\boldsymbol{\nu})+\psi_{2}(\boldsymbol{\nu})\right]=\bar{\epsilon}_{s}$, the function $\psi_{1}(\boldsymbol{\nu})+$ $\psi_{2}(\boldsymbol{\nu})$ can be reduced such that $E\left[\psi_{1}(\boldsymbol{\nu})+\psi_{2}(\boldsymbol{\nu})\right]=\vartheta$ for any $0 \leq \vartheta \leq \bar{\epsilon}_{s}$. Therefore, the interval $\left[0, E\left[\psi_{1}(\boldsymbol{\nu})+\psi_{2}(\boldsymbol{\nu})\right]\right]$ is no longer required, leading to the result in (4.39).

Thus for a given $0 \leq \epsilon_{s} \leq 1$, it is clear that the common outage capacity $\mathcal{C}_{o}\left(\mathbf{P}_{a v}, \epsilon_{p}, \epsilon_{s}\right)$ can be implicitly obtained when the minimum common outage probability for a given rate $\mathbf{r}_{o}$ is computed where the optimal power control is applied. In other words, for any rate vector $\mathbf{r}_{o}$ such that $\mathbf{r}_{o} \in \mathcal{C}_{o}\left(\mathbf{P}_{a v}, \epsilon_{p}, \epsilon_{s}\right)$ if and only if $\operatorname{Pr}_{\min }\left(\mathbf{P}_{a v}, \epsilon_{p}, \mathbf{r}_{o}\right) \leq \epsilon_{s}$

From Definitions 4.3.3 - 4.3.5, deriving for the optimal power control for the common outage probability region is identical to solving for the optimal power policy that maximizes the common usage probability in the set $\overline{\mathcal{O}}_{C}\left(\mathbf{P}_{a v}, \epsilon_{p}, \mathbf{r}_{o}\right)$. Hence, we have to solve

$$
\max _{\bar{\varepsilon}_{s}} \bar{\varepsilon}_{s} \quad \text { s.t. } \bar{\varepsilon}_{s} \in \overline{\mathcal{O}}_{C}\left(\mathbf{P}_{a v}, \epsilon_{p}, \mathbf{r}_{o}\right) .
$$


With a given rate vector $\mathbf{r}_{o}$, define the set $\mathcal{Q}_{C}\left(\mathbf{r}_{o}\right)=\left\{\left(\bar{\varepsilon}_{s}, \overline{\mathbf{P}}, \varepsilon_{p}\right) \mid \bar{\varepsilon}_{s} \in \overline{\mathcal{O}}_{C}\left(\overline{\mathbf{P}}, \varepsilon_{p}, \mathbf{r}_{o}\right)\right\}$. Due to the convexity of the set $\mathcal{Q}_{C}\left(\mathbf{r}_{o}\right)$ (the proof is in Appendix C.6), the problem (4.41) can be rewritten as

$$
\max _{\left(\bar{\varepsilon}_{s}, \overline{\mathbf{P}}, \varepsilon_{p}\right) \in \mathcal{Q}_{C}\left(\mathbf{r}_{o}\right)} \bar{\varepsilon}_{s} \quad \text { s.t. } \quad \varepsilon_{p} \leq \epsilon_{p} \text { and } \overline{\mathbf{P}} \preceq \mathbf{P}_{a v}
$$

By Proposition 4.3.1, a probability $\bar{\varepsilon}_{s}$ is in $\overline{\mathcal{O}}_{C}\left(\mathbf{P}_{a v}, \epsilon_{p}, \mathbf{r}_{o}\right)$, if and only if there exists $\left(\psi_{k}(\boldsymbol{\nu}), p_{k}(\boldsymbol{\nu})\right)$ that satisfies the constraints (2) to (8) in Lemma. 4.3 .1 and $\bar{\varepsilon}_{s}=$ $E\left[\psi_{1}(\boldsymbol{\nu})+\psi_{2}(\boldsymbol{\nu})\right]$. So, we have to solve

$$
\begin{array}{ll}
\max _{\mathbf{p}_{k}(\boldsymbol{\nu}) \succeq \mathbf{0}, \psi_{k}(\boldsymbol{\nu}) \geq 0} & E\left[\psi_{1}(\boldsymbol{\nu})+\psi_{2}(\boldsymbol{\nu})\right] \\
\text { s.t. } & E\left[\sum_{k=1}^{4} \psi_{k}(\boldsymbol{\nu}) \mathbf{p}_{k}(\boldsymbol{\nu})\right] \preceq \mathbf{P}_{a v}, \\
& E\left[\psi_{1}(\boldsymbol{\nu})+\psi_{3}(\boldsymbol{\nu})\right] \geq 1-\epsilon_{p} \\
& \varphi_{k}(\boldsymbol{\nu})\left[\mathcal{P}_{p}(g)-\boldsymbol{\beta}^{T} \mathbf{p}_{k}(\boldsymbol{\nu})\right] \geq 0, \quad k=1,3, \\
& 1-\sum_{k=1}^{4} \psi_{k}(\boldsymbol{\nu})=0, \\
& \mathbf{r}_{o} \in \mathcal{C}_{M A C}\left(\boldsymbol{\nu}, \mathbf{p}_{k}(\boldsymbol{\nu})\right), \quad k=1,2 .
\end{array}
$$

We will solve the optimization Problem (4.43) by a functional optimization technique similar to [25]. The Lagrangian corresponding to (4.43) can be written as follows:

$$
\begin{aligned}
& l\left(\boldsymbol{\nu}, \mathbf{p}_{1}(\boldsymbol{\nu}), \mathbf{p}_{2}(\boldsymbol{\nu}), \psi_{k}(\boldsymbol{\nu}), \lambda, s, \varphi(\boldsymbol{\nu}), \Gamma(\boldsymbol{\nu})\right) \\
& =\psi_{1}(\boldsymbol{\nu})\left[1-\boldsymbol{\lambda}^{T} \mathbf{p}_{1}(\boldsymbol{\nu})+s+\varphi_{1}(\boldsymbol{\nu})\left(\mathcal{P}_{p}(g)-\boldsymbol{\beta}^{T} \mathbf{p}_{1}(\boldsymbol{\nu})\right)-\Gamma(\boldsymbol{\nu})\right] \\
& +\psi_{2}(\boldsymbol{\nu})\left[1-\boldsymbol{\lambda}^{T} \mathbf{p}_{2}(\boldsymbol{\nu})-\Gamma(\boldsymbol{\nu})\right] \\
& +\psi_{3}(\boldsymbol{\nu})\left[-\boldsymbol{\lambda}^{T} \mathbf{p}_{3}(\boldsymbol{\nu})+s+\varphi_{3}(\boldsymbol{\nu})\left(\mathcal{P}_{p}(g)-\boldsymbol{\beta}^{T} \mathbf{p}_{1}(\boldsymbol{\nu})\right)-\Gamma(\boldsymbol{\nu})\right] \\
& +\psi_{4}(\boldsymbol{\nu})\left[-\boldsymbol{\lambda}^{T} \mathbf{p}_{4}(\boldsymbol{\nu})-\Gamma(\boldsymbol{\nu})\right]+\Gamma(\boldsymbol{\nu})
\end{aligned}
$$

where $\boldsymbol{\lambda}, s, \varphi(\boldsymbol{\nu}), \Gamma(\boldsymbol{\nu})$ are the nonnegative Lagrange multipliers corresponding to constraints $(4.43 \mathrm{~b})$ to $(4.43 \mathrm{e})$. With these Lagrange multipliers, we can write the KKT conditions corresponding to Problem (4.43) which is provided in Appendix C.7.

As $\mathbf{r}_{o}$ is a function of $\mathbf{p}_{k}^{*}$, the region $\mathbf{r}_{o} \in \mathcal{C}_{M A C}\left(\boldsymbol{\nu}, \mathbf{p}_{k}^{*}(\boldsymbol{\nu})\right)$ can be described 
by $2^{M}-1$ constraints for each $k$ and it is not easy to visualize. Instead of using the KKT conditions directly, we can observe that for given $\boldsymbol{\lambda}^{* T}, s^{*}$ and $\varphi_{3}^{*}(\boldsymbol{\nu})$ with arbitrary $\psi_{k}^{*}(\boldsymbol{\nu})$, the optimization problem can be decomposed to four subproblems. Each of them corresponds to the optimal $\mathbf{p}_{k}^{*}$.

$$
\text { Subproblem for } \mathbf{p}_{4}^{*}: \max _{\mathbf{p}_{4}(\boldsymbol{\nu})}-\lambda^{* T} \mathbf{p}_{4}(\boldsymbol{\nu})-\Gamma^{*}(\boldsymbol{\nu}) \text {. }
$$

$$
\begin{aligned}
& \text { Subproblem for } \mathbf{p}_{3}^{*}: \max _{\mathbf{p}_{3}(\boldsymbol{\nu})}-\left[\boldsymbol{\lambda}^{* T}+\varphi^{*}(\boldsymbol{\nu}) \boldsymbol{\beta}^{T}\right] \mathbf{p}_{3}(\boldsymbol{\nu}) \\
& +\left[s^{*}-\Gamma^{*}(\boldsymbol{\nu})+\varphi_{3}^{*}(\boldsymbol{\nu}) P_{p}(g)\right] \\
& \text { s.t. } \varphi_{3}^{*}(\boldsymbol{\nu})\left[P_{p}(g)-\boldsymbol{\beta}^{T} \mathbf{p}_{3}(\boldsymbol{\nu})\right]=0 .
\end{aligned}
$$

Subproblem for $\mathbf{p}_{2}^{*}: \max _{\mathbf{p}_{2}(\boldsymbol{\nu})}-\boldsymbol{\lambda}^{* T} \mathbf{p}_{2}(\boldsymbol{\nu})-\Gamma^{*}(\boldsymbol{\nu})$

s.t. $\mathbf{r}_{o} \in \mathcal{C}_{M A C}\left(\boldsymbol{\nu}, \mathbf{p}_{2}(\boldsymbol{\nu})\right)$.

$$
\begin{gathered}
\text { Subproblem for } \mathbf{p}_{1}^{*}: \begin{array}{r}
\max _{\mathbf{p}_{1}(\boldsymbol{\nu})}-\left[\boldsymbol{\lambda}^{* T}+\varphi_{1}^{*}(\boldsymbol{\nu}) \boldsymbol{\beta}^{T}\right] \mathbf{p}_{1}(\boldsymbol{\nu}) \\
\quad+\left[s^{*}-\Gamma^{*}(\boldsymbol{\nu})+\varphi_{1}^{*}(\boldsymbol{\nu}) P_{p}(g)\right] \\
\text { s.t. } \mathbf{r}_{o} \in \mathcal{C}_{M A C}\left(\boldsymbol{\nu}, \mathbf{p}_{1}(\boldsymbol{\nu})\right), \\
\varphi_{1}^{*}(\boldsymbol{\nu})\left[P_{p}(g)-\boldsymbol{\beta}^{T} \mathbf{p}_{1}(\boldsymbol{\nu})\right]=0 .
\end{array}
\end{gathered}
$$

In Appendix C.8, we will solve for the optimal power $\mathbf{p}_{k}(\boldsymbol{\nu})$, providing that the corresponding $\psi_{k}^{*}(\boldsymbol{\nu})>0$. Essentially Lemma 3.3 in [14] serves as the main tool for obtaining $\mathbf{p}_{1}^{*}(\boldsymbol{\nu})$ and $\mathbf{p}_{2}^{*}(\boldsymbol{\nu})$. We then restate Lemma 3.3 in [14] as follows:

Lemma 4.3.2. Let $\tilde{\mathscr{G}}(f)$ be a contra-polymatroid. Then the points $v(\pi)$ where $\pi$ is the permutation on $E$ are precisely the vertices of $\tilde{\mathscr{G}}(f)$. Moreover, if $\boldsymbol{\Lambda}$ is a given vector in $\mathbf{R}_{+}^{M}$ then a solution of the optimization problem

$$
\min _{\mathbf{p}_{1}(\boldsymbol{\nu})} \boldsymbol{\Lambda}^{T} \cdot \mathbf{x} \quad \text { s.t. } \mathbf{x} \in \tilde{\mathscr{G}}(f)
$$

is attained at a point $v\left(\pi^{*}\right)$, where $\pi^{*}$ is any permutation such that $\Lambda_{\pi^{*}(1)} \geq \cdots \geq$ $\Lambda_{\pi^{*}(M)}$. Conversely, if $f$ is a set function and $v(\pi) \in \tilde{\mathscr{G}}(f)$ for every permutation $\pi$. Then $\tilde{\mathscr{G}}(f)$ is a contra-polymatroid.

Define $\boldsymbol{\eta} \triangleq\left[\eta_{1}, \ldots, \eta_{M}\right]$ and $\eta_{i}=\lambda_{i}^{*}+\varphi_{1}^{*}(\boldsymbol{\nu}) \beta_{i}$. Then, we summarize the expression of the optimal solution in Proposition 4.3.2 below 
Proposition 4.3.2. Provided that $\psi_{k}^{*}(\boldsymbol{\nu})>0$, the corresponding $\mathbf{p}_{k}^{*}(\boldsymbol{\nu})$ can be expressed as follows: $\mathbf{p}_{4}^{*}(\boldsymbol{\nu})=\mathbf{p}_{3}^{*}(\boldsymbol{\nu})=\mathbf{0}$.

For $\mathbf{p}_{2}^{*}(\boldsymbol{\nu})$, we have

$$
p_{2, \pi_{2}(i)}^{*}= \begin{cases}\frac{1}{h_{\pi_{2}(i)}}\left[\exp \left(r_{o, \pi_{2}(i)}\right)-1\right], & i=1 \\ \frac{1}{h_{\pi_{2}(i)}}\left[\exp \left(\sum_{m=1}^{i} r_{o, \pi_{2}(m)}\right)-\exp \left(\sum_{m=1}^{i-1} r_{o, \pi_{2}(m)}\right)\right], & i=2, \ldots, M\end{cases}
$$

where the permutation $\pi_{2}$ satisfies $\frac{\lambda_{\pi_{2}(1)}^{*}}{h_{\pi_{2}(1)}} \geq \ldots \geq \frac{\lambda_{\pi_{2}(M)}^{*}}{h_{\pi_{2}(M)}}$.

For $\mathbf{p}_{1}^{*}(\boldsymbol{\nu})$, we have

$$
p_{1, \pi_{1}(i)}^{*}= \begin{cases}\frac{1}{h_{\pi_{1}(i)}}\left[\exp \left(r_{o, \pi_{1}(i)}\right)-1\right], & i=1 \\ \frac{1}{h_{\pi_{1}(i)}}\left[\exp \left(\sum_{m=1}^{i} r_{o, \pi_{1}(m)}\right)-\exp \left(\sum_{m=1}^{i-1} r_{o, \pi_{1}(m)}\right)\right], & i=2, \ldots, M \\ \varphi_{1}^{*}(\boldsymbol{\nu})\left[\boldsymbol{\beta}^{T} \mathbf{p}_{1}^{*}-\mathcal{P}_{p}(g)\right]=0 & \end{cases}
$$

where the permutation $\pi_{1}$ satisfies $\frac{\eta_{\pi(1)}}{h_{\pi(1)}} \geq \ldots \geq \frac{\eta_{\pi(M)}}{h_{\pi(M)}}$.

Proof. See Appendix C.8.

Remark 4.3.2. The characteristics of $\mathbf{p}_{1}^{*}$ and $\mathbf{p}_{2}^{*}$ are quite similar to the delaylimited solution in [19] because the definition of $\mathbf{p}_{1}^{*}$ and $\mathbf{p}_{2}^{*}$ restrict these power control schemes to always satisfy $\mathbf{r}_{o} \in \mathcal{C}\left(\boldsymbol{\nu}, \mathbf{p}_{k}\right)$ for $k=1,2$. Nevertheless, the solutions for $\mathbf{p}_{1}^{*}$ and $\mathbf{p}_{2}^{*}$ are different in the sense that power price to pay in $\mathbf{p}_{2}^{*}$ is $\boldsymbol{\lambda}^{*}$ while power price to pay in $\mathbf{p}_{1}^{*}$ is $\boldsymbol{\eta} \succeq \boldsymbol{\lambda}^{*}$. It is because the PU must not be in outage when the power control scheme $\mathbf{p}_{1}^{*}$ is applied, according to the definition. By this limitation, the secondary network has to pay an additional cost for $\mathbf{p}_{1}^{*}$ in order to ensure the PU's target rate for that channel realization.

For the optimal $\psi_{k}^{*}(\boldsymbol{\nu})$, we define four benefit functions corresponding to $\mathbf{p}_{k}^{*}(\boldsymbol{\nu})$ as follows: $\mathscr{B}_{1}^{\text {com }}=1-\boldsymbol{\lambda}^{* T} \mathbf{p}_{1}^{*}(\boldsymbol{\nu})+s^{*}, \mathscr{B}_{2}^{\text {com }}=1-\boldsymbol{\lambda}^{* T} \mathbf{p}_{2}^{*}(\boldsymbol{\nu}), \mathscr{B}_{3}^{\text {com }}=-\boldsymbol{\lambda}^{* T} \mathbf{p}_{3}^{*}(\boldsymbol{\nu})+s^{*}$ and $\mathscr{B}_{4}^{\text {com }}=-\boldsymbol{\lambda}^{* T} \mathbf{p}_{4}^{*}(\boldsymbol{\nu})$. Note that the superscript 'com' stands for 'common outage'. According to the KKT conditions, we have that, $\psi_{k}^{*}(\boldsymbol{\nu})>0$ if $\mathscr{B}_{k}^{c o m}=\max _{j} \mathscr{B}_{j}^{\text {com }}$ and $\psi_{k}^{*}(\boldsymbol{\nu})=0$ if $\mathscr{B}_{k}^{c o m} \leq \max _{j} \mathscr{B}_{j}^{\text {com }}$ for all $k$. As we consider continuous fading 
channels, the condition that $\mathscr{B}_{k}=\mathscr{B}_{j}$ for $j \neq k$ happens with probability of zero measure. Hence, there is only one $\psi_{k}^{*}(\boldsymbol{\nu})=1$ corresponding to the maximum $\mathscr{B}_{j}$.

Theorem 4.3.1. The optimal solution of (4.43) is $\mathbf{P}^{*}(\boldsymbol{\nu})=\sum_{k=1}^{4} X_{\psi_{k}^{*}}(\boldsymbol{\nu}) \mathbf{p}_{k}^{*}(\boldsymbol{\nu})$, where $\mathbf{p}_{k}^{*}(\boldsymbol{\nu})$ follows from Proposition 4.3.2. $\psi_{k}^{*}(\boldsymbol{\nu})$ is determined by

$$
\psi_{k}^{*}(\boldsymbol{\nu})=\left\{\begin{array}{c}
1, \quad \mathscr{B}_{k}=\max _{j} \mathscr{B}_{j} \\
0, \quad \mathscr{B}_{k}<\max _{j} \mathscr{B}_{j}
\end{array} \text { for } k=1,2,3,4\right.
$$

$\lambda^{* T}$ and $s^{*}$ are the solutions to $E\left[\mathbf{P}^{*}(\boldsymbol{\nu})\right] \preceq \mathbf{P}_{a v}$ and $E\left[\psi_{1}^{*}(\boldsymbol{\nu})+\psi_{3}^{*}(\boldsymbol{\nu})\right] \geq 1-\epsilon_{p}$.

Remark 4.3.3. It can be noticed that there are at most three power strategies for each channel realization $\boldsymbol{\nu}$ as the optimal solutions $\mathbf{p}_{3}^{*}=\mathbf{p}_{4}^{*}=\mathbf{0}$. First, let us consider the three possible scenarios from Table 4.1. In the first case, it implies that PU faces outage, although $M$ SUs are turned off. Therefore, it is impossible to make $\psi_{1}^{*}$ and $\psi_{3}^{*}$ be positive. The second case means that SU can avoid causing an outage either by setting $\mathbf{P}^{*}=\mathbf{0}$ or by setting $\mathbf{P}^{*}=\mathbf{p}_{1}^{*}$. Otherwise, they may apply strategy $\mathbf{p}_{2}^{*}$ when they decide to cause an outage to PU. For the last case, SUs do not cause any outage to $\mathrm{PU}$ despite using policy $\mathbf{p}_{2}^{*}$. That is why $\psi_{2}^{*}=0$ due to the definition in (4.37). Mathematically, it happens when $\mathbf{p}_{1}^{*}=\mathbf{p}_{2}^{*}$, i.e. when $\varphi_{1}^{*}(\boldsymbol{\nu})=0$. From Table. 4.1, we can also show that the solution in [44] is a special case when $M=1$.

Table 4.1: Three possible cases in common outage problem for a given $\boldsymbol{\nu}$

\begin{tabular}{cll}
\hline Cases & Candidate non-negative $\psi_{k}$ & Power allocation policy \\
\hline$r_{p}(\boldsymbol{\nu}, \mathbf{0})<r_{p}^{0}$ & $\psi_{2}^{*}, \psi_{4}^{*}$ & $\mathbf{p}_{2}^{*}, \mathbf{p}_{4}^{*}$ \\
$r_{p}(\boldsymbol{\nu}, \mathbf{0}) \geq r_{p}^{0}$ and $r_{p}\left(\boldsymbol{\nu}, \mathbf{p}_{2}^{*}\right)<r_{p}^{0}$ & $\psi_{1}^{*}, \psi_{2}^{*}, \psi_{3}^{*}$ & $\mathbf{p}_{1}^{*}, \mathbf{p}_{2}^{*}, \mathbf{p}_{3}^{*}$ \\
$r_{p}(\boldsymbol{\nu}, \mathbf{0}) \geq r_{p}^{0}$ and $r_{p}\left(\boldsymbol{\nu}, \mathbf{p}_{2}^{*}\right) \geq r_{p}^{0}$ & $\psi_{1}^{*}, \psi_{3}^{*}$ & $\mathbf{p}_{1}^{*}=\mathbf{p}_{2}^{*}, \mathbf{p}_{3}^{*}$ \\
\hline \hline
\end{tabular}

\subsubsection{Individual outage capacity}

This section will investigate how to achieve each boundary point of individual outage capacity regarding to Definition 4.3.2. To apply the probabilistic power allocation technique, we have to know all the situation that can happen in each $\boldsymbol{\nu}$ and then 
assign the corresponding weighting function. We can define that by the aid of the information below.

- Let $0 \leq M_{o} \leq M$ and $\mathscr{S}_{n}$ be the set of SUs that do not face individual outage (subscript $n$ stands for non-outage). Then, if there are $M_{o}$ users that face outage, the remaining $M_{n}=M-M_{o}$ SUs are not in outage. In other words, if $r_{i}(\boldsymbol{\nu}, \mathbf{P}(\boldsymbol{\nu})) \geq r_{o, i}$, then $i \in \mathscr{S}_{n}$. Otherwise, $i \in \mathcal{E} \backslash \mathscr{S}_{n}$. It is obvious that there are $2^{M}$ possible sets of $\mathscr{S}_{n}$. Hence, for $0 \leq j \leq 2^{M}-1$, we can fix the index of SUs in $\mathscr{S}_{n}(j)$ for each $j$. Also, let the cardinality of $\mathscr{S}_{n}(j)$ be $N_{j}$. Hence, the cardinality of $\mathscr{S}_{n}(0)$ is zero.

- For convenience, we define $\boldsymbol{\xi}(j)=[\xi(j, 1), \ldots, \xi(j, M)]^{T}$ as the binary expansion of $j$. For each vector $\xi(j)$, if $\xi(j, i)=0$, then $\mathrm{SU}_{i}$ is in outage $\left(i \notin \mathscr{S}_{n}(j)\right)$. Otherwise, $i \in \mathscr{S}_{n}(j)$ and $\xi(j, i)=1$ ( $\mathrm{SU}_{i}$ is not in outage).

- Furthermore, the power control policy in the set $\mathscr{S}_{n}(j)$ may or may not cause an outage to the PU. In other words, we still have two subcases which are $r_{p}(\boldsymbol{\nu}, \mathbf{P}(\boldsymbol{\nu}))<r_{p}^{0}$ or $r_{p}(\boldsymbol{\nu}, \mathbf{P}(\boldsymbol{\nu})) \geq r_{p}^{0}$.

- Therefore, we have

$$
\begin{aligned}
\operatorname{Pr}\left\{r_{i}(\boldsymbol{\nu}, \mathbf{P}(\boldsymbol{\nu})) \geq r_{o, i}\right\}= & \sum_{j=1}^{2^{M}-1} \operatorname{Pr}\left\{i \in \mathscr{S}_{n}(j)\right\} \\
= & \sum_{j=1}^{2^{M}-1} \operatorname{Pr}\left\{i \in \mathscr{S}_{n}(j), r_{p}(\boldsymbol{\nu}, \mathbf{P}(\boldsymbol{\nu})) \geq r_{p}^{0}\right\} \\
& +\sum_{j=1}^{2^{M}-1} \operatorname{Pr}\left\{i \in \mathscr{S}_{n}(j), r_{p}(\boldsymbol{\nu}, \mathbf{P}(\boldsymbol{\nu}))<r_{p}^{0}\right\}
\end{aligned}
$$

With the information above, we can define weighting function $\zeta_{k}(\boldsymbol{\nu}, j)$ as follows:

$$
\begin{array}{ll}
\zeta_{1}(\boldsymbol{\nu}, j)= & \operatorname{Pr}\left\{r_{p}(\boldsymbol{\nu}, \mathbf{P}(\boldsymbol{\nu})) \geq r_{p}^{0}, \mathscr{S}_{n}(j) \mid \boldsymbol{\nu}\right\} \\
\zeta_{2}(\boldsymbol{\nu}, j)= & \operatorname{Pr}\left\{r_{p}(\boldsymbol{\nu}, \mathbf{P}(\boldsymbol{\nu}))<r_{p}^{0}, \mathscr{S}_{n}(j) \mid \boldsymbol{\nu}\right\}
\end{array}
$$

Note that if $i \in \mathscr{S}_{n}(j)$, then $\operatorname{Pr}\left\{r_{i}(\boldsymbol{\nu}, \mathbf{P}(\boldsymbol{\nu})) \geq r_{o, i}, \mathscr{S}_{n}(j)\right\}=E\left[\zeta_{1}(\boldsymbol{\nu}, j)+\zeta_{2}(\boldsymbol{\nu}, j)\right]$. 
For the corresponding $\mathbf{p}_{k}(\boldsymbol{\nu}, j)$ for each $\zeta_{k}(\boldsymbol{\nu}, j)$, they can be expressed as follows:

$$
\begin{array}{ll}
\mathbf{p}_{1}(\boldsymbol{\nu}, j)= & E\left[\mathbf{P}(\boldsymbol{\nu}) \mid r_{p}(\boldsymbol{\nu}, \mathbf{p}(\boldsymbol{\nu})) \geq r_{p}^{0}, \mathscr{S}_{n}(j), \boldsymbol{\nu}\right] \\
\mathbf{p}_{2}(\boldsymbol{\nu}, j)= & E\left[\mathbf{P}(\boldsymbol{\nu}) \mid r_{p}(\boldsymbol{\nu}, \mathbf{p}(\boldsymbol{\nu}))<r_{p}^{0}, \mathscr{S}_{n}(j), \boldsymbol{\nu}\right]
\end{array}
$$

Lemma 4.3.3. To achieve $\mathcal{C}_{o}\left(\mathbf{P}_{a v}, \epsilon_{p}, \boldsymbol{\epsilon}_{s}\right)$, there exists a power policy $\mathbf{P}(\boldsymbol{\nu})$ of the form $\mathbf{P}(\boldsymbol{\nu})=\sum_{j=0}^{2^{M}-1} \sum_{k=1}^{2} X_{\zeta_{k}(\boldsymbol{\nu}, j)}(\boldsymbol{\nu}) \mathbf{p}_{k}(\boldsymbol{\nu}, j)$ where

- (1) $\sum_{j: i \in \mathscr{S}_{n}(j)} E\left[\zeta_{1}(\boldsymbol{\nu}, j)+\zeta_{2}(\boldsymbol{\nu}, j)\right] \geq 1-\epsilon_{s, i}, \forall i$,

(2) $E\left[\sum_{j=0}^{2^{M}-1} \sum_{k=1}^{2} \zeta_{k}(\boldsymbol{\nu}, j) \mathbf{p}_{k}(\boldsymbol{\nu}, j)\right] \preceq \mathbf{P}_{a v}$,

(3) $\sum_{j=0}^{2^{M}-1} E\left[\zeta_{1}(\boldsymbol{\nu}, j)\right] \geq 1-\epsilon_{p}$,

(4) $\zeta_{1}(\boldsymbol{\nu}, j)\left[\mathcal{P}_{p}(g)-\boldsymbol{\beta}^{T} \mathbf{p}_{1}(\boldsymbol{\nu}, j)\right] \geq 0, \forall j$,

(5) $1-\sum_{j=0}^{2^{M}-1} \sum_{k=1}^{2} \zeta_{k}(\boldsymbol{\nu}, j)=0, \quad \forall j, k=1,2$,

(6) $\mathbf{p}_{k}(\boldsymbol{\nu}, j) \succeq \mathbf{0}, \quad \forall j, k=1,2$,

(7) $\zeta_{k}(\boldsymbol{\nu}, j) \geq 0, \quad \forall j, k=1,2$,

(8) $\boldsymbol{\xi}(j) \mathbf{r}_{o} \in \mathcal{C}_{M A C}\left(\boldsymbol{\nu}, \mathbf{p}_{k}(\boldsymbol{\nu}, j)\right), \quad \forall j, k=1,2$.

Appendix C.9 provides the details of proof of Lemma 4.3.3.

Similar to Lemma 4.3.1, Lemma 4.3.3 reveals that the optimal power scheme $\mathbf{P}^{*}(\boldsymbol{\nu})$ is randomized amongst the $2 \cdot 2^{M}$ deterministic power policies $\mathbf{p}_{k}(\boldsymbol{\nu}, j)$ and we can solve for $\mathbf{p}_{k}(\boldsymbol{\nu})$ and $\psi_{k}(\boldsymbol{\nu})$ accordingly. Following the same procedure as in Section 4.3.2, we will prove that the individual outage capacity region is implicitly obtained by deriving the individual outage probability region for each given rate vector via Definition 4.3.6 to 4.3.7 and Proposition 4.3.3 as follows.

Definition 4.3.6. The outage probability vector $\boldsymbol{\epsilon}_{s}$ is in the individual outage probability set $\mathcal{O}_{I}\left(\mathbf{P}_{a v}, \epsilon_{p}, \mathbf{r}_{o}\right)$ if and only if the rate vector $\mathbf{r}_{o} \in \mathcal{C}_{o}\left(\mathbf{P}_{a v}, \epsilon_{p}, \boldsymbol{\epsilon}_{s}\right)$.

Definition 4.3.7. The usage probability vector $\overline{\boldsymbol{\epsilon}}_{s}$ is in the individual usage probability set $\overline{\mathcal{O}}_{I}\left(\mathbf{P}_{a v}, \epsilon_{p}, \mathbf{r}_{o}\right)$ if and only if the rate vector $\mathbf{r}_{o} \in \mathcal{C}_{o}\left(\mathbf{P}_{a v}, \epsilon_{p}, \mathbf{1}-\overline{\boldsymbol{\epsilon}}_{s}\right)$. 
Proposition 4.3.3. The individual usage probability set $\overline{\mathcal{O}}_{I}\left(\mathbf{P}_{a v}, \epsilon_{p}, \mathbf{r}_{o}\right)$ is equivalently given by

$$
\overline{\mathcal{O}}_{I}\left(\mathbf{P}_{a v}, \epsilon_{p}, \mathbf{r}_{o}\right)=\bigcup_{\left(\zeta_{k}(\boldsymbol{\nu}, j), p_{k}(\boldsymbol{\nu}, j)\right) \in \mathcal{F}}\left[\Omega_{1}, \ldots, \Omega_{M}\right]
$$

where $\mathcal{F} \triangleq\left\{\left(\zeta_{k}(\boldsymbol{\nu}, j), p_{k}(\boldsymbol{\nu}, j)\right)\right.$ : the constraints (2) to (8) in Lemma. 4.3.3 $\}$ and $\Omega_{i}=\sum_{j: i \in \mathscr{S}_{n}(j)} E\left[\zeta_{1}(\boldsymbol{\nu}, j)+\zeta_{2}(\boldsymbol{\nu}, j)\right]$.

Proof. The individual usage probability vector $\left[\bar{\epsilon}_{s, 1}, \ldots, \bar{\epsilon}_{s, M}\right]$ is in the individual usage probability set if and only if the rate vector $\mathbf{r}_{o} \in \mathcal{C}_{o}\left(\mathbf{P}_{a v}, \epsilon_{p}, \mathbf{1}-\boldsymbol{\epsilon}_{s}\right)$, which is true if and only if all constraints in Lemma. 4.3.3 are satisfied. Using the same idea from the proof in Proposition 4.3.1, we can obtain the result in Proposition 4.3.3.

With Definition 4.3.7 and Proposition 4.3.3, we have shown that, with a given $\boldsymbol{\epsilon}_{s}$, $\mathcal{C}_{o}\left(\mathbf{P}_{a v}, \epsilon_{p}, \boldsymbol{\epsilon}_{s}\right)$ can be implicitly acquired with the boundary of the usage probability region $\overline{\mathcal{O}}_{I}\left(\mathbf{P}_{a v}, \epsilon_{p}, \mathbf{r}_{o}\right)$ for a given $\mathbf{r}_{o}$ is solved via the optimal power allocation because $\mathbf{r}_{o} \in \mathcal{C}_{o}\left(\mathbf{P}_{a v}, \epsilon_{p}, \boldsymbol{\epsilon}_{s}\right)$ if and only if $\left[1-\epsilon_{s, 1}, \ldots, 1-\epsilon_{s, M}\right] \in \overline{\mathcal{O}}_{I}\left(\mathbf{P}_{a v}, \epsilon_{p}, \mathbf{r}_{o}\right)$.

Define the set $\mathcal{Q}_{I}\left(\mathbf{r}_{o}\right)=\left\{\left(\overline{\boldsymbol{\varepsilon}}_{s}, \overline{\mathbf{P}}, \varepsilon_{p}\right) \mid \overline{\boldsymbol{\varepsilon}}_{s} \in \overline{\mathcal{O}}_{I}\left(\overline{\mathbf{P}}, \varepsilon_{p}, \mathbf{r}_{o}\right)\right\}$ and prove in Appendix C.10 that $\mathcal{Q}_{I}\left(\mathbf{r}_{o}\right)$ is a convex set and the individual usage probability region $\overline{\mathcal{O}}_{I}\left(\mathbf{P}_{a v}, \epsilon_{p}, \mathbf{r}_{o}\right)$ is convex.

From the convexity of $\overline{\mathcal{O}}_{I}\left(\mathbf{P}_{a v}, \epsilon_{p}, \mathbf{r}_{o}\right)$, the usage probability vector $\bar{\varepsilon}_{s}$ will be on the boundary surface of $\overline{\mathcal{O}}_{I}\left(\mathbf{P}_{a v}, \epsilon_{p}, \mathbf{r}_{o}\right)$ if and only if it is the solution to

$$
\max _{\bar{\varepsilon}_{s} \in \overline{\mathcal{O}}_{I}\left(\mathbf{P}_{a v}, \epsilon_{p}, \mathbf{r}_{o}\right)} \boldsymbol{\mu}^{T} \cdot \bar{\varepsilon}_{s}
$$

for some nonnegative $\boldsymbol{\mu}=\left[\mu_{1}, \ldots, \mu_{M}\right]^{T}$. Together with the convexity of the set $\mathcal{Q}_{I}\left(\mathbf{r}_{o}\right)$ and Proposition 4.3.3, it implies that we must solve

$$
\begin{array}{ll}
\max _{\mathbf{p}_{k}(\boldsymbol{\nu}, j), \zeta_{1}(\boldsymbol{\nu}, j)} & \sum_{j=1}^{2^{M}-1} \sum_{i \in \mathscr{S}_{n}(j)} \mu_{i} E\left[\zeta_{1}(\boldsymbol{\nu}, j)+\zeta_{2}(\boldsymbol{\nu}, j)\right] \\
\text { s.t. } & E\left[\sum_{j=0}^{2^{M}-1} \sum_{k=1}^{2} \zeta_{k}(\boldsymbol{\nu}, j) \mathbf{p}_{k}(\boldsymbol{\nu}, j)\right] \preceq \mathbf{P}_{a v}
\end{array}
$$




$$
\begin{aligned}
& \sum_{j=0}^{2^{M}-1} E\left[\zeta_{1}(\boldsymbol{\nu}, j)\right] \geq 1-\epsilon_{p} \\
& \zeta_{1}(\boldsymbol{\nu}, j)\left[\mathcal{P}_{p}(g)-\boldsymbol{\beta}^{T} \mathbf{p}_{1}(\boldsymbol{\nu}, j)\right] \geq 0, \forall j, k \\
& 1-\sum_{j=0}^{2^{M}-1} \sum_{k=1}^{2} \zeta_{k}(\boldsymbol{\nu}, j)=0 \\
& \mathbf{p}_{k}(\boldsymbol{\nu}, j) \succeq 0, \quad k=1,2 \\
& \zeta_{k}(\boldsymbol{\nu}, j) \geq 0, \quad k=1,2 \\
& \boldsymbol{\xi}(j) \mathbf{r}_{o} \in \mathcal{C}_{M A C}\left(\boldsymbol{\nu}, \mathbf{p}_{k}(\boldsymbol{\nu}, j)\right), \quad \forall j, k .
\end{aligned}
$$

The variable $\mu_{i} \geq 0$ represents the reward if the target rate of $\mathrm{SU}_{i}$ is met. By varying the given nonnegative vector $\boldsymbol{\mu}$, the derived optimal power solutions will achieve all the boundary points of $\overline{\mathcal{O}}_{I}\left(\mathbf{P}_{a v}, \epsilon_{p}, \mathbf{r}_{o}\right)$.

To solve for the optimal solution, we first write the corresponding Lagrangian.

$$
\begin{aligned}
& l\left(\boldsymbol{\nu}, \mathbf{p}_{k}(\boldsymbol{\nu}, j), \zeta_{k}(\boldsymbol{\nu}, j), \lambda, s, \phi(\boldsymbol{\nu}, j), \Gamma(\boldsymbol{\nu})\right) \\
& =\sum_{j=0}^{2^{M}-1} \zeta_{1}(\boldsymbol{\nu}, j)\left[N_{j}\left(\sum_{i \in \mathscr{S}_{n}(j)} \mu_{i}\right)-\boldsymbol{\lambda}^{T} \mathbf{p}_{1}(\boldsymbol{\nu}, j)+s+\phi(\boldsymbol{\nu}, j)\left(\mathcal{P}_{p}(g)-\boldsymbol{\beta}^{T} \mathbf{p}_{1}(\boldsymbol{\nu}, j)\right)-\Gamma(\boldsymbol{\nu})\right] \\
& +\sum_{j=0}^{2^{M}-1} \zeta_{2}(\boldsymbol{\nu}, j)\left[N_{j}\left(\sum_{i \in \mathscr{S}_{n}(j)} \mu_{i}\right)-\boldsymbol{\lambda}^{T} \mathbf{p}_{2}(\boldsymbol{\nu}, j)-\Gamma(\boldsymbol{\nu})\right]+\Gamma(\boldsymbol{\nu})
\end{aligned}
$$

where $\lambda, s, \phi(\boldsymbol{\nu}, j)$, and $\Gamma(\boldsymbol{\nu})$ are the Lagrange multipliers corresponding to constraints $(4.56 \mathrm{~b})$ to $(4.56 \mathrm{e})$. The KKT necessary and sufficient conditions corresponding to Problem (4.56) are provided in Appendix C.11.

Similar to the solving procedure in Section 4.3.2, the problem can be decomposed to $2 \cdot 2^{M}$ subproblems each corresponding to $\zeta_{k}^{*}(\boldsymbol{\nu}, j)$. Therefore, we can write subproblems regarding to $\mathbf{p}_{2}^{*}(\boldsymbol{\nu}, j)$ and $\mathbf{p}_{1}^{*}(\boldsymbol{\nu}, j)$ as follows

$$
\begin{aligned}
& \text { Subproblem for } \mathbf{p}_{2}(\boldsymbol{\nu}, j): \max _{\mathbf{p}_{2}(\boldsymbol{\nu}, j)} N_{j}\left(\sum_{i \in \mathscr{S}_{n}(j)} \mu_{i}\right)-\boldsymbol{\lambda}^{* T} \mathbf{p}_{2}(\boldsymbol{\nu}, j)-\Gamma^{*}(\boldsymbol{\nu}) \\
& \text { s.t. } \boldsymbol{\xi}(j) \mathbf{r}_{o} \in \mathcal{C}_{M A C}\left(\boldsymbol{\nu}, \mathbf{p}_{2}(\boldsymbol{\nu}, j)\right) .
\end{aligned}
$$




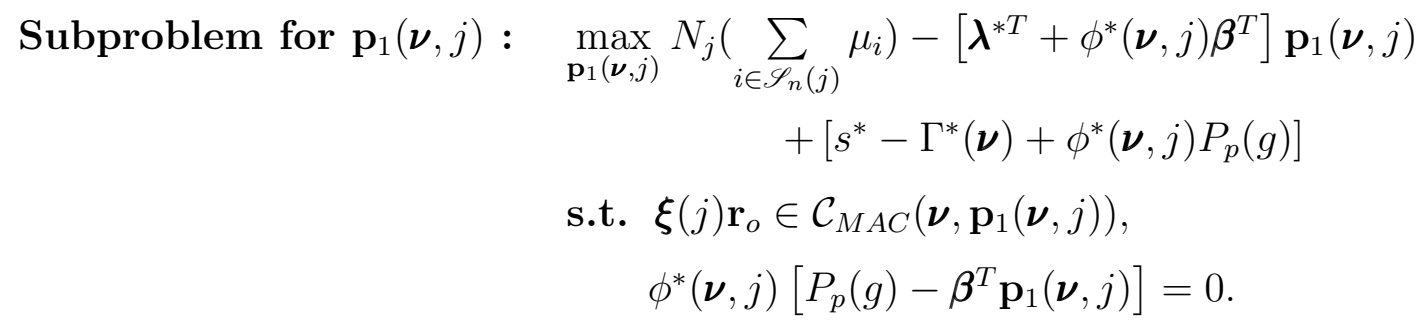

Define $\boldsymbol{\eta}(j)=\left[\eta_{1}(j), \ldots, \eta_{M}(j)\right]$ and $\eta_{i}(j)=\lambda_{i}^{*}+\phi^{*}(\boldsymbol{\nu}, j) \beta_{i}$. With the aid of Lemma 4.3.2, we can find the optimal solution for $\mathbf{p}_{2}^{*}(\boldsymbol{\nu}, j)$ and $\mathbf{p}_{1}^{*}(\boldsymbol{\nu}, j)$ as summarized in Proposition 4.3 .4 by solving each subproblem.

Proposition 4.3.4. Providing that $\zeta_{k}^{*}(\boldsymbol{\nu}, j)>0$, the corresponding $\mathbf{p}_{k}^{*}(\boldsymbol{\nu}, j)$ for the set $\mathscr{S}_{n}(j)$ for $j=0, \ldots, 2^{M}-1$ can be expressed as follows: For $\mathbf{p}_{2}^{*}(\boldsymbol{\nu}, j)$,

$$
\begin{array}{cl}
p_{2, i}^{*}(\boldsymbol{\nu}, j)=0, & \forall i \notin \mathscr{S}_{n}(j) \\
p_{2, \pi_{2, j}(l)}^{*}= \begin{cases}\frac{1}{h_{\pi_{2, j}(l)}}\left[\exp \left(r_{o, \pi_{2, j}(l)}\right)-1\right], & l=1 \\
\frac{1}{h_{\pi_{2, j}(l)}}\left[\exp \left(\sum_{m=1}^{i} r_{o, \pi_{2, j}(m)}\right)-\exp \left(\sum_{m=1}^{i-1} r_{o, \pi_{2, j}(m)}\right)\right], & l=2, \ldots, N_{j}\end{cases}
\end{array}
$$

where the permutation function $\pi_{2, j}($.$) is for the N_{j} S U$ s in the subset $\mathscr{S}_{n}(j)$ and satisfies $\frac{\lambda_{\pi_{2, j}(1)}}{h_{\pi_{2, j}(1)}} \geq \ldots \geq \frac{\lambda_{\pi_{2, j}\left(N_{j}\right)}}{\bar{h}_{\pi_{2, j}\left(N_{j}\right)}}$. For $\mathbf{p}_{1}^{*}(\boldsymbol{\nu}, j)$,

$$
\begin{aligned}
& p_{1, i}^{*}(\boldsymbol{\nu}, j)=0, \quad \forall i \notin \mathscr{S}_{n}(j) \\
& p_{1, \pi_{1, j}(l)}^{*}= \begin{cases}\frac{1}{h_{\pi_{1, j}(l)}}\left[\exp \left(r_{o, \pi_{1, j}(l)}\right)-1\right], & l=1 \\
\frac{1}{h_{\pi_{1, j}(l)}}\left[\exp \left(\sum_{m=1}^{i} r_{o, \pi_{1, j}(m)}\right)-\exp \left(\sum_{m=1}^{i-1} r_{o, \pi_{1, j}(m)}\right)\right], & l=2, \ldots, N_{j}\end{cases} \\
& \phi^{*}(\boldsymbol{\nu}, j)\left[\boldsymbol{\beta}^{T} \mathbf{p}_{1}^{*}(\boldsymbol{\nu}, j)-P_{p}(g)\right]=0
\end{aligned}
$$

where the permutation function $\pi_{1, j}($.$) is for the N_{j} S U s$ in the subset $\mathscr{S}_{n}(j)$ and satisfies $\frac{\eta_{\pi_{1, j}(1)}(j)}{\bar{h}_{\pi_{1, j}(1)}} \geq \ldots \geq \frac{\eta_{\pi_{1, j}\left(N_{j}\right)}(j)}{\bar{h}_{\pi_{1, j}\left(N_{j}\right)}}$.

Proof. Please refer to Appendix C.12.

Remark 4.3.4. For the optimal $\mathbf{p}_{2}^{*}(\boldsymbol{\nu}, j)$, the solution is similar to $\mathbf{p}_{1}^{*}(\boldsymbol{\nu}, j)$ except one needs to use $\frac{\lambda_{\pi_{2, j}(1)}^{*}}{h_{\pi_{2, j}(l)}}$ instead of $\frac{\eta_{\pi_{1, j}(j)}}{h_{\pi_{1, j}(l)}}$ for all users in the subset $\mathscr{S}_{n}(j)$. The 
interpretation is that for the strategy $\mathbf{p}_{1}^{*}(\boldsymbol{\nu}, j)$, SUs have to avoid disturbing PU and therefore consider the term $\eta_{\pi(l)}(j)$ as a power price, whereas the interference cost term $\phi^{*}(\boldsymbol{\nu}, j) \beta_{i}$ is neglected in strategy $\mathbf{p}_{2}^{*}(\boldsymbol{\nu}, j)$.

Furthermore, it can be observed that for strategy $\mathbf{p}_{1}^{*}(\boldsymbol{\nu}, j)$ for all $j$ is the solution for minimizing individual outage probability subject to short-term interference power constraint with threshold $\mathcal{P}_{p}(g)$. That is why the solution $\mathbf{p}_{1}^{*}(\boldsymbol{\nu}, j)$ is the same as in [53], where the short-term interference threshold is fixed. For $\mathbf{p}_{2}^{*}(\boldsymbol{\nu}, j)$ for all $j$, the solution is similar to [22] which there is no interference constraint imposed in that problem.

Remark 4.3.5. When $j=0, \mathscr{S}_{n}(j)$ is an empty set as there is no $i \in \mathscr{S}_{n}(0)$, so all SUs are turned OFF. If $\mathscr{S}_{n}(j)=\mathcal{E}$, then all SUs turn ON and the solution is the same as delay-limited case similar to [19].

For the optimal solution of $\zeta_{k}^{*}(\boldsymbol{\nu}, j)$. For simplicity, we define the benefit functions $\mathscr{B}_{k}^{i n d}(\boldsymbol{\nu}, j)$ corresponding to the power policy $\mathbf{p}_{k}^{*}(\boldsymbol{\nu}, j)$ as follows: $\mathscr{B}_{1}^{\text {ind }}(\boldsymbol{\nu}, j)=$ $N_{j}\left(\sum_{i \in \mathscr{S}_{n}(j)} \mu_{i}\right)-\boldsymbol{\lambda}^{* T} \mathbf{p}_{1}^{*}(\boldsymbol{\nu}, j)+s^{*}$ and $\mathscr{B}_{2}^{i n d}(\boldsymbol{\nu}, j)=N_{j}\left(\sum_{i \in \mathscr{S}_{n}(j)} \mu_{i}\right)-\boldsymbol{\lambda}^{* T} \mathbf{p}_{2}^{*}(\boldsymbol{\nu}, j)$. Note that the superscript 'ind' represents 'individual outage'. Applying KKT conditions and the fact that we consider continuous fading channels, the condition that $\mathscr{B}_{k}^{\text {ind }}(\boldsymbol{\nu}, j)=\mathscr{B}_{k^{\prime}}^{\text {ind }}\left(\boldsymbol{\nu}, j^{\prime}\right)$ for $j \neq j^{\prime}$ or for $k \neq k^{\prime}$ happens with probability of measure zero. Therefore there is only one $\zeta_{k}^{*}(\boldsymbol{\nu}, j)=1$ which corresponds to the maximum $\mathscr{B}_{k}^{\text {ind }}(\boldsymbol{\nu}, j)$ and this leads to Theorem 4.3 .2 as follows.

Theorem 4.3.2. The optimal solution of (4.56) is $\mathbf{P}^{*}(\boldsymbol{\nu})=\sum_{k=1}^{2} \sum_{j=0}^{2^{M}-1} X_{\zeta_{k}^{*}(j)}(\boldsymbol{\nu}) \mathbf{p}_{k}^{*}(\boldsymbol{\nu}, j)$, where $\mathbf{p}_{k}^{*}(\boldsymbol{\nu}, j)$ follows from Proposition 4.3.4. $\zeta_{k}^{*}(\boldsymbol{\nu}, j)$ is determined by

$$
\zeta_{k}^{*}(\boldsymbol{\nu}, j)=\left\{\begin{array}{cc}
1, & \mathscr{B}_{k}(\boldsymbol{\nu}, j)=\max _{j^{\prime}, k^{\prime}} \mathscr{B}_{k^{\prime}}\left(\boldsymbol{\nu}, j^{\prime}\right) \\
0, & \mathscr{B}_{k}(\boldsymbol{\nu}, j)<\max _{j^{\prime}, k^{\prime}} \mathscr{B}_{k^{\prime}}\left(\boldsymbol{\nu}, j^{\prime}\right)
\end{array} \quad \text { for } k=1,2 \text { and } j=0, \ldots, 2^{M}-1\right.
$$

where $\boldsymbol{\lambda}^{* T}$ and $s^{*}$ are the solutions to $E\left[\mathbf{P}^{*}(\boldsymbol{\nu})\right] \preceq \mathbf{P}_{a v}$ and $\sum_{j=0}^{2^{M}-1} E\left[\zeta_{1}^{*}(\boldsymbol{\nu}, j)\right] \geq 1-\epsilon_{p}$. 


\subsection{Simulation results}

In this section, we present some numerical results on the performance of the proposed optimal power policies. All channels involved are presumed to undergo Rayleigh fading and the corresponding channel power gains are taken to be exponentially distributed with unit mean. Noises at the PR and the SBS are assumed to be equal and AWGN with unit variance, i.e. $N_{0}=1$ or $(0 \mathrm{~dB})$. Unless specified otherwise, the constant power $P_{c}$ for the primary ON-OFF power policy is set to be $15 \mathrm{~dB}$. when the $\mathrm{PU}$ is $\mathrm{ON}$. The primary outage probability is set to be 0.1 . For convenience, we further assume that all secondary transmitters are identical. The simulation results are based on a typical Monte-Carlo method averaged over $10^{5}$ channel realizations.

\subsubsection{Ergodic capacity results}

In this part, all channel power gains are taken to be exponentially distributed with unit mean. Fig. 4.2 exhibits the plot of SU ergodic sum uplink capacity against average power budget per SU with varying number of users $M$ when PU's target rate is fixed at $r_{p}^{0}=0.3$ nats per channel use. With an increasing average power $P_{a v, i}$, ergodic sum capacity at the SBS increases for both with and without POC as expected. However, the POC becomes the dominant constraint when the average power budget is raised further, leading to the significant decrement in the ergodic sum capacity compared to the case without POC.

Using the solution in Section 4.2.2, we sketch the ergodic capacity region for $M=2$ as shown in Fig.4.3. The figure compares the region for both with and without POC for a fixed target rate which we provide two values of fixed target rates, i.e. when $r_{p}^{0}=0.3$ and when $r_{p}^{0}=1.0$. The figure reveals that when POC is imposed, the ergodic capacity region is smaller than the one without POC. The explanation is that SUs cannot transmit with as much power as they would like due to the service quality protection in the primary link. The region is even smaller if $r_{p}^{0}$ is raised further as POC becomes stricter. Note that the region for without POC, one can notice that the region when $r_{p}^{0}=1.0$ is slightly larger than that of $r_{p}^{0}=0.3$. This is because, when $r_{p}^{0}=1.0$ is large, the probability that PU will turn 


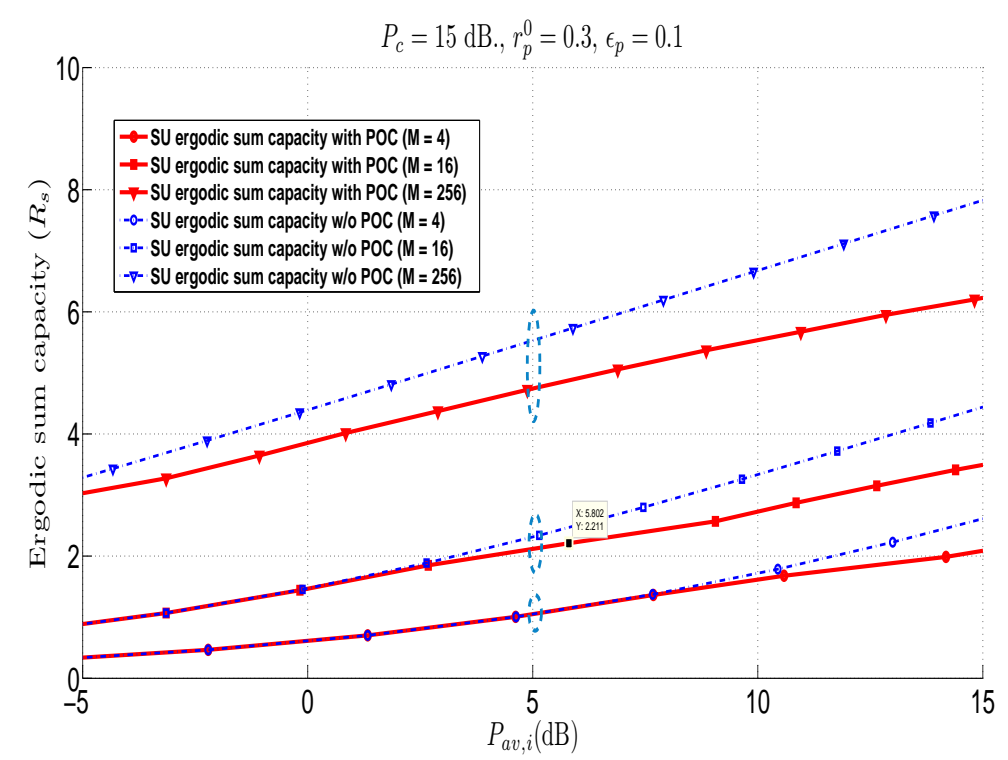

Figure 4.2: SU ergodic sum capacity against average power budget per user : $P_{c}=15$ $\mathrm{dB} ., r_{p}^{0}=0.3$, and $\epsilon_{p}=0.1$

off is higher by the definition of ON-OFF power control and thus it causes lower interference to the secondary network than the PU in the case of $r_{p}^{0}=0.3$ does. Note that in order to generate each point on the boundary region, we first set the value of the pre-defined vector $\boldsymbol{\mu}=\left[\mu_{1}, \mu_{2}\right]^{T}=[1, \tan \theta]$, where $\theta \in\left[0,90^{\circ}\right]$. Then, a point on the boundary can be generated by the optimal power control policy corresponding to each specific value of $\theta$, as it is varied between $\theta=0$ to $\theta=90^{\circ}$. This way we can obtain the entire curve of each boundary.

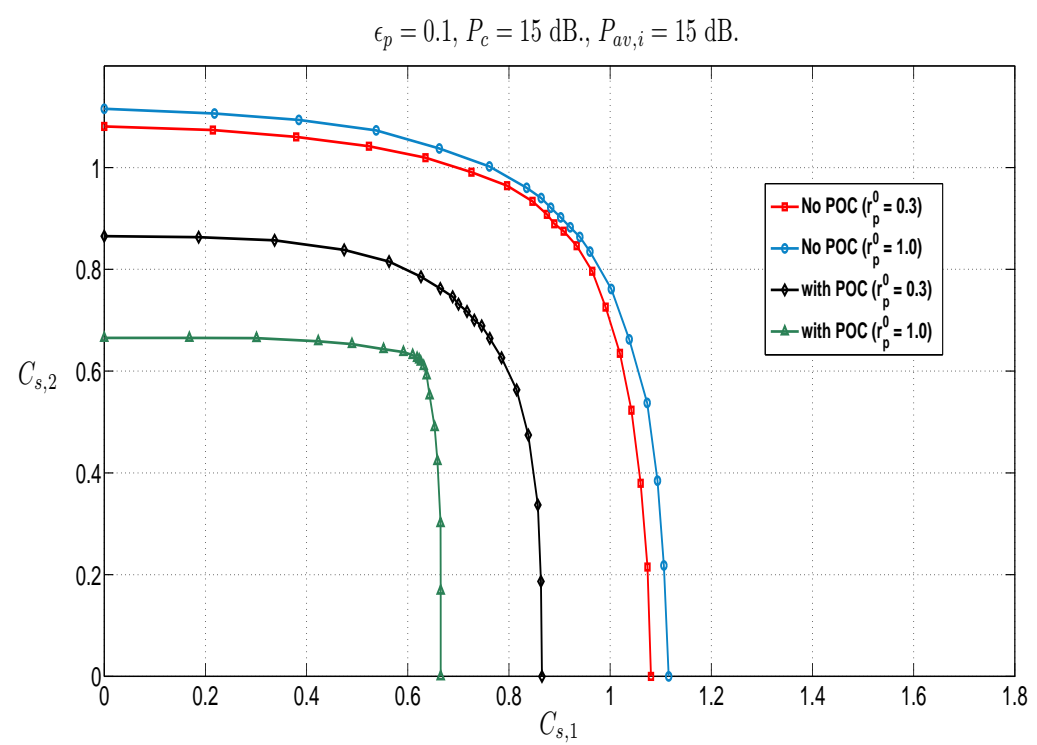

Figure 4.3: Ergodic-capacity region corresponding to feasible power control policy for the problem without POC and with POC: $\epsilon_{p}=0.1, P_{c}=15 \mathrm{~dB} ., P_{a v, i}=15 \mathrm{~dB}$. 


\subsubsection{Outage probability results}

In this part, we assume that the direct channel power gains, i.e. $g$ and $h_{i}$, are exponentially distributed with unit mean whereas the cross channel power gains, i.e. $\alpha$ and $\beta_{i}$, are exponentially distributed with mean 0.5 .

First, we look at how the POC affects the common outage probability. Fig. 4.4 illustrates the common outage probability performance of the optimal power control for maximizing the common usage probability subject to ATPC and POC when $M=2, \mathbf{r}_{o}$ to be $[0.3,0.3]$ and fixed $\epsilon_{p}=0.1$ for various PU's target rates, i.e. $r_{p}^{0}=0.15,0.25,0.5$. As expected, when the target rate is raised, SUs face more common outage for a given average power constraint. For a fixed PU's target rate $r_{p}^{0}=0.25$, Fig. 4.5 depicts the common outage performance with several PU outage probability thresholds, i.e. $\epsilon_{p}=0.03,0.08,0.1$, and also includes the case that POC is discarded from the problem $\left(\epsilon_{p}=1\right)$. It can be noticed that common outage probability can be reduced further if PU outage probability threshold is increased, i.e. the constraint becomes more lenient.

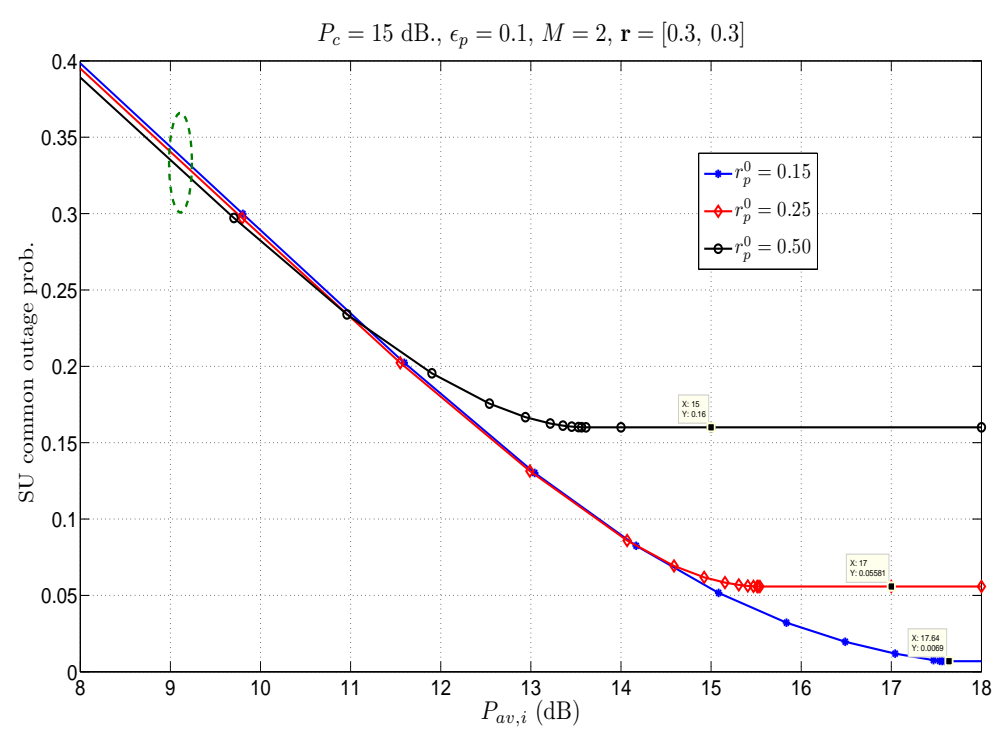

Figure 4.4: Common outage probability against average power constraint per user: $P_{c}=15 \mathrm{~dB} ., \epsilon_{p}=0.1, M=2, \mathbf{r}=\left[\begin{array}{ll}0.3 & 0.3\end{array}\right]$

Next, Fig. 4.6 is used to illustrate the effect of the number of secondary transmitters in the C-MAC network for the common outage problem against the individual average power constraint $P_{a v, i}$. We set the target rate vectors such that each 


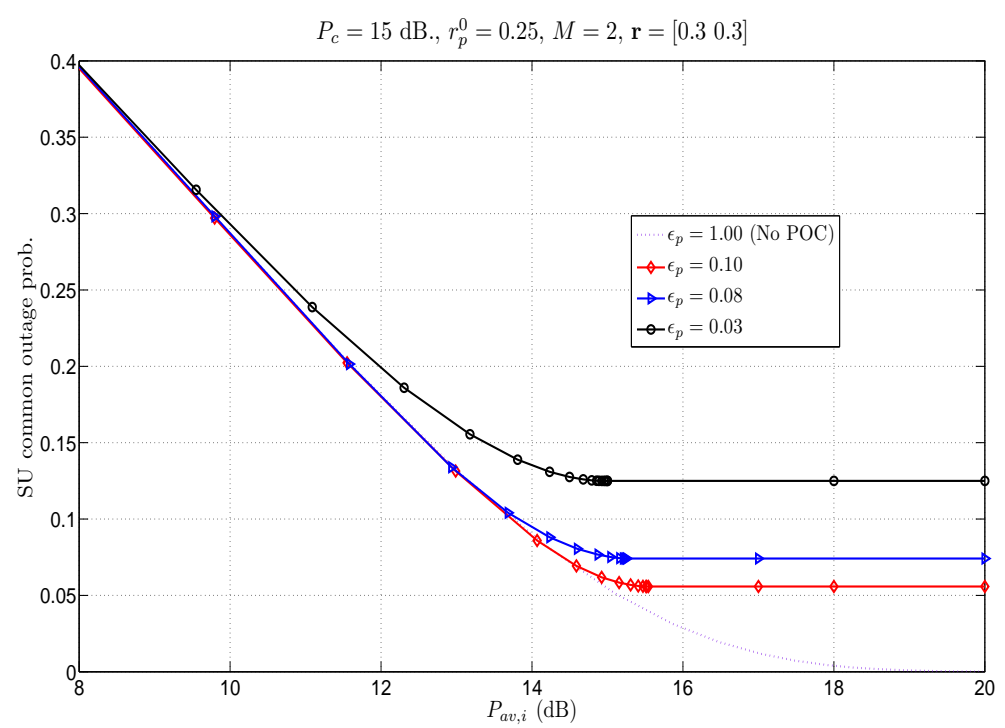

Figure 4.5: Common outage probability against average power constraint per user: $P_{c}=15 \mathrm{~dB} ., r_{p}^{0}=0.25, M=2, \mathbf{r}=\left[\begin{array}{ll}0.3 & 0.3\end{array}\right]$

$r_{o, i}=0.3$ for $M=1,2,3$. With other parameters fixed, we can observe that, the secondary network faces a higher common outage as the number of secondary transmitters is raised. The reason is twofold. First, $M$ SUs must either support all the individual target rates or turn off in the common usage probability maximization problem, so the problem becomes stricter with an increasing $M$. Second, the user who decodes first must encounter the interference from the users who decode after him. Therefore, with the increment in $M$, the interference signals are magnified and each SU requires more power to support their target rates. The individual usage probability maximization problem is a more relaxed problem compared to the common usage maximization problem as we do not have to satisfy all the outage probability constraints for the target rate vector. The results in Fig.4.7 affirms this fact. Fig. 4.7(a) reveals that, when all SUs are identical, the achievable individual outage probability is significantly lower than the achievable common outage probability because the secondary network in the individual outage case has more alternatives for its power allocation policy while the secondary network in the common outage tends to keep silent, making the actual PU outage probability in the individual outage case always higher than that of the common outage case as shown in Fig.4.7(b).

Fig.4.8 demonstrates the effect of POC on the individual outage probability. By 


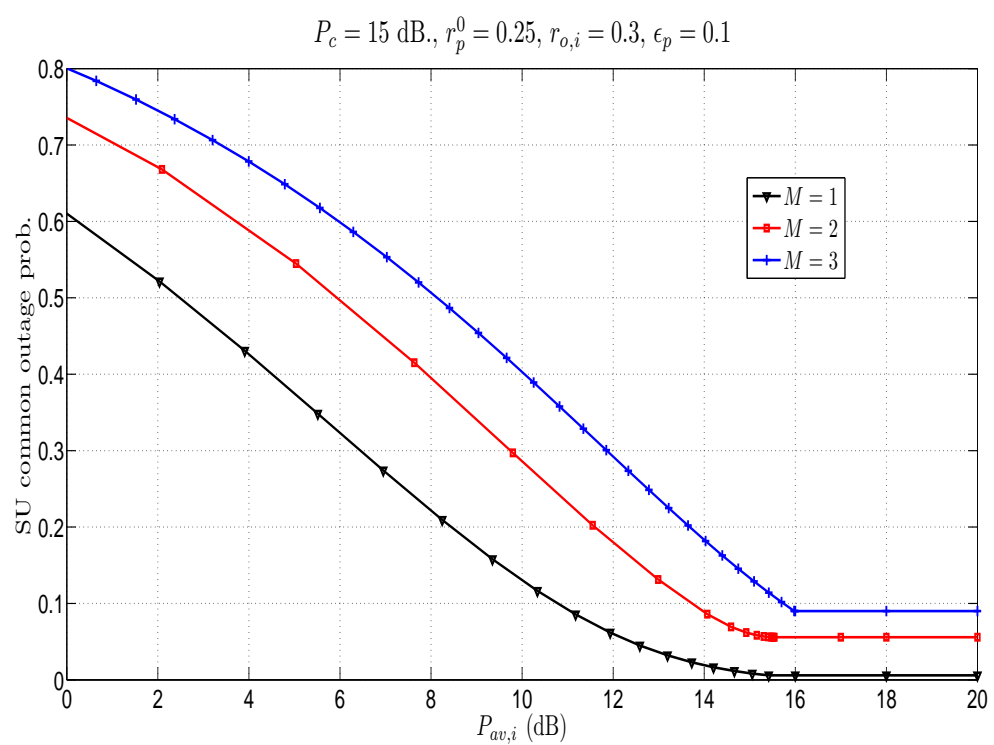

Figure 4.6: Common outage probability against average power constraint per user with $M=1$ and $M=2: P_{c}=15 \mathrm{~dB} ., r_{p}^{0}=0.25, r_{o, i}=0.3$

using $r_{p}^{0}=0.25, \epsilon_{p}=0.1$ as a reference, if we increase the $\mathrm{PU}$ target rate (from $r_{p}^{0}=0.25$ to $r_{p}^{0}=0.5$ ) or decrease the outage probability threshold (from $\epsilon_{p}=0.1$ to $\left.\epsilon_{p}=0.05\right)$, POC will become stricter and thus lessen the outage performance in each secondary transmitter. Finally, we consider the effect of the decoding order strategy on each $\mathrm{SU}_{i}$ 's individual outage probability performance when $M=3$. In Fig.4.9, the results depict the outage probability performance both in the secondary network (in Fig.4.9(a)) and in the primary link (in Fig.4.9(b)) due to the optimal decoding order and another suboptimal decoding strategy which always decodes $\mathrm{SU}_{1}$ first and $\mathrm{SU}_{3}$ last. As expected, Fig.4.9(a) shows that $\mathrm{SU}_{3}$ faces an outage least frequently under the same $P_{a v, i}$ whereas the individual outage probability for $\mathrm{SU}_{1}$ is the largest among the users. Also, the outage probability in $\mathrm{SU}_{3}$ of the suboptimal decoding order case is substantially reduced compare to that of the optimal decoding order case. The reason is that the suboptimal decoding strategy always places $\mathrm{SU}_{3}$ in the highest priority by decoding last. Hence, $\mathrm{SU}_{3}$ does not require power to cope with the interference from any other users while $\mathrm{SU}_{1}$ has to combat interferences from all other users. The performance for the user whose message is decoded first for the suboptimal strategy is, of course, worse than that of the optimal. However, it can be seen from Fig.4.9(b) that the suboptimal decoding order creates an outage event in the primary link more often than the optimal decoding order does. It is 


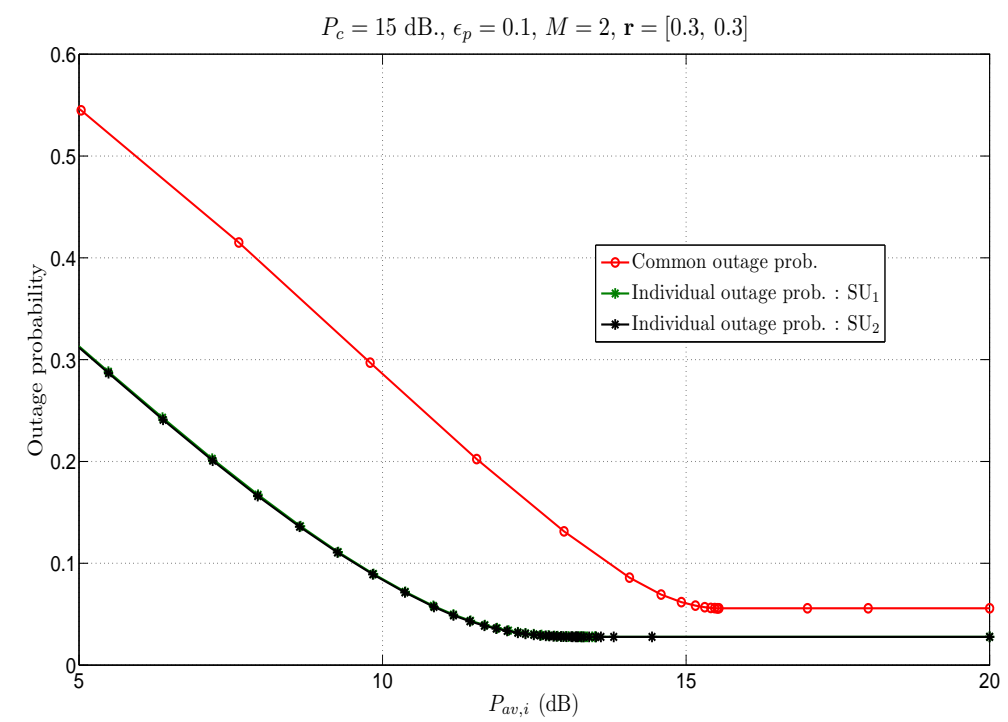

(a) Outage probability performance for common outage and individual outage problem

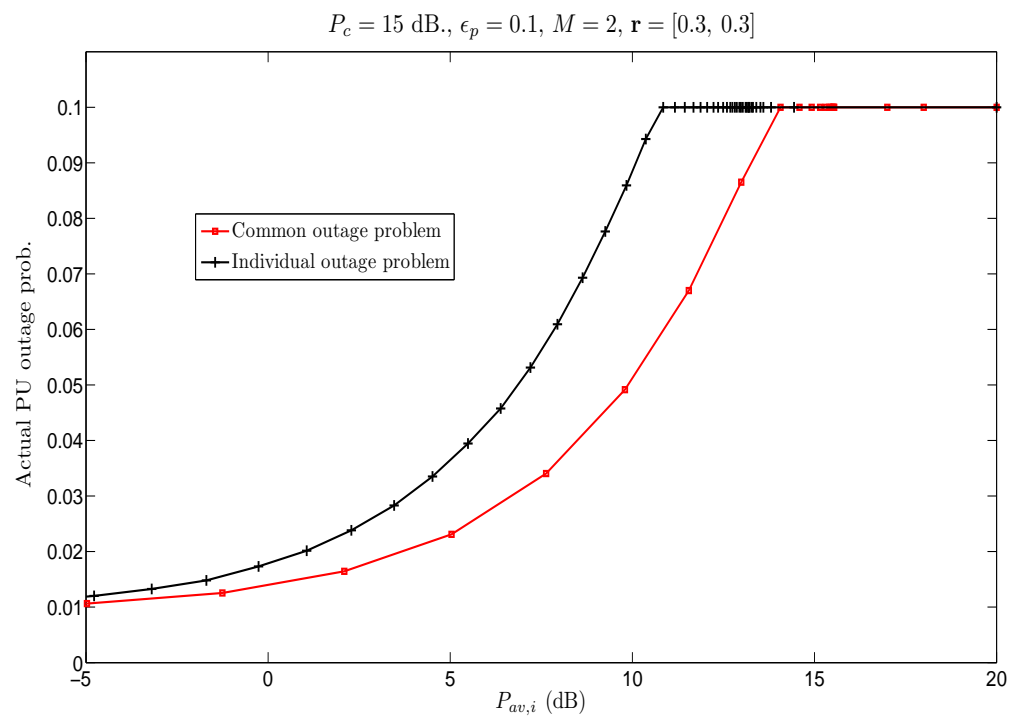

(b) Actual PU's outage probability performance due to optimal power policies in common outage and individual outage problem

Figure 4.7: Common outage probability and individual outage probability performance against average power constraint per user: $P_{c}=15 \mathrm{~dB} ., r_{p}^{0}=0.25, M=2$, $\mathbf{r}=\left[\begin{array}{ll}0.3 & 0.3\end{array}\right]$ 


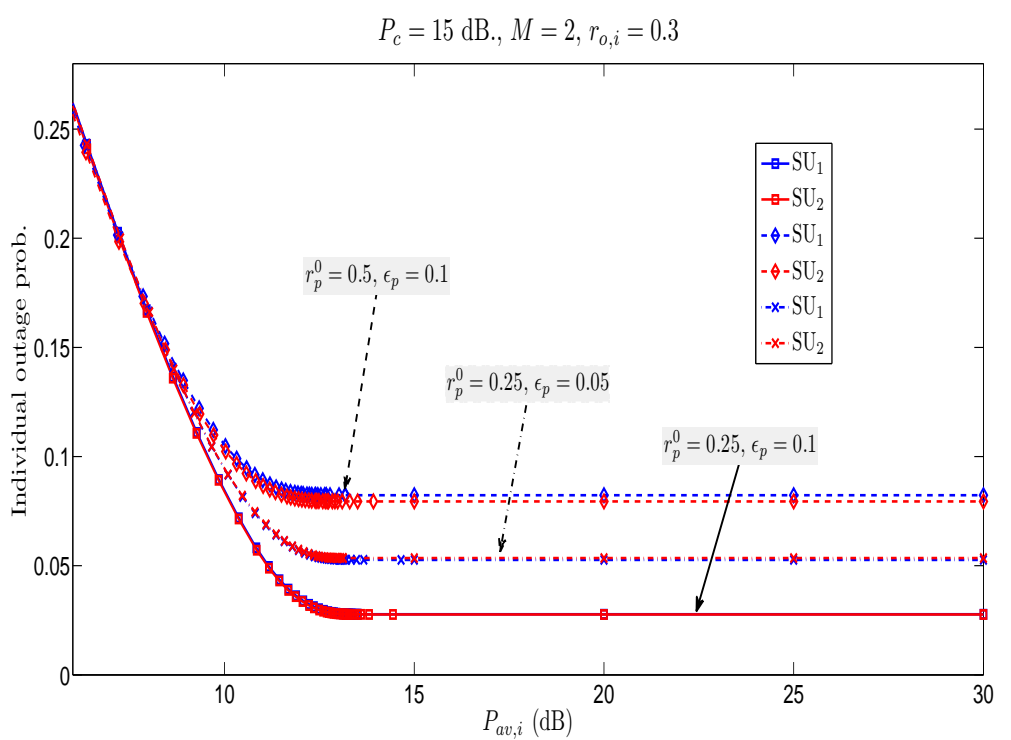

Figure 4.8: Individual outage probability performance against average power constraint per user: $P_{c}=15 \mathrm{~dB} ., M=2, r_{o, i}=0.3$

because the suboptimal decoding strategy provides less options for SU power control policies. For the optimal power strategy, SUs can select $N_{j}$ users to transmit, leading to $\sum_{N_{j}=0}^{M}\left(\begin{array}{c}M \\ N_{j}\end{array}\right)=2^{M}$ possible options. Also, among the selected $N_{j}$ users, there are $N_{j}$ ! permutations for decoding. Thus, the suboptimal method suggests that the complexity can be reduced if the decoding priority among the user is fixed beforehand while sacrificing the outage probability performance in some lower-priority secondary transmitters and outage performance in the PU's link accordingly.

\subsection{Conclusions}

In this chapter, we have studied the optimal power allocation problem in a fading multiple-access cognitive radio network sharing the same frequency band with a delay-sensitive primary user. Under a primary user outage probability constraint and individual average power constraints for each secondary user, we have derived the optimal power policies for achieving points the ergodic capacity region, common outage capacity region and the individual outage capacity region, with full CSI at all secondary transmitters for continuous fading channels. We have shown that the problems involved in this chapter can be solved by using probabilistic power control techniques. For power control for each case, we have shown that the successive 


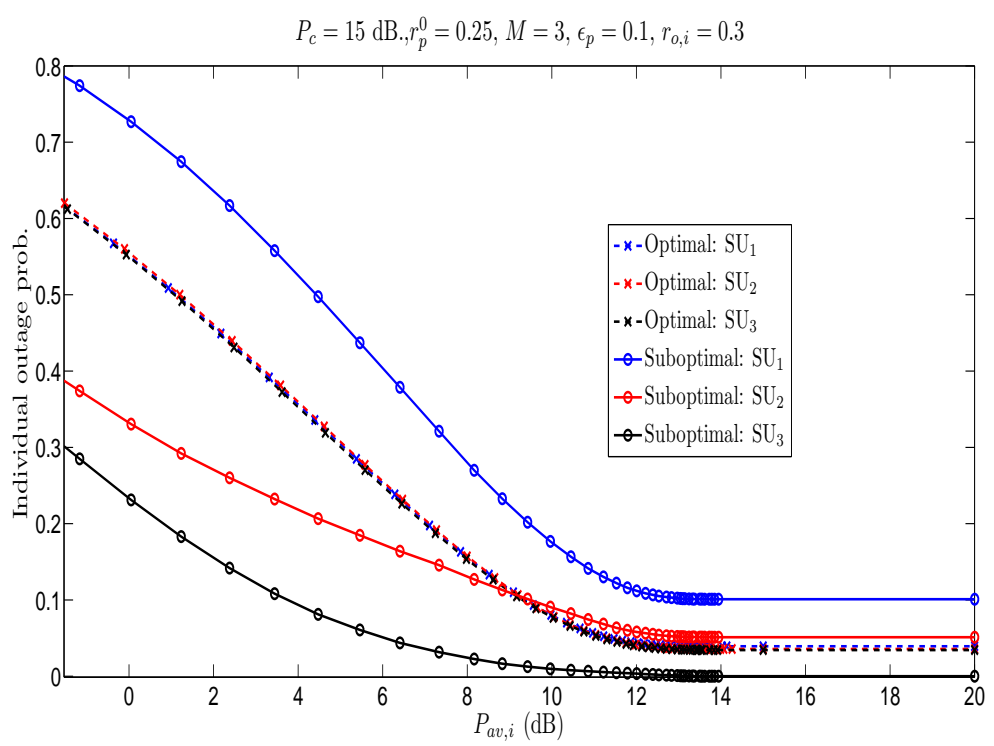

(a) Individual outage probability performance

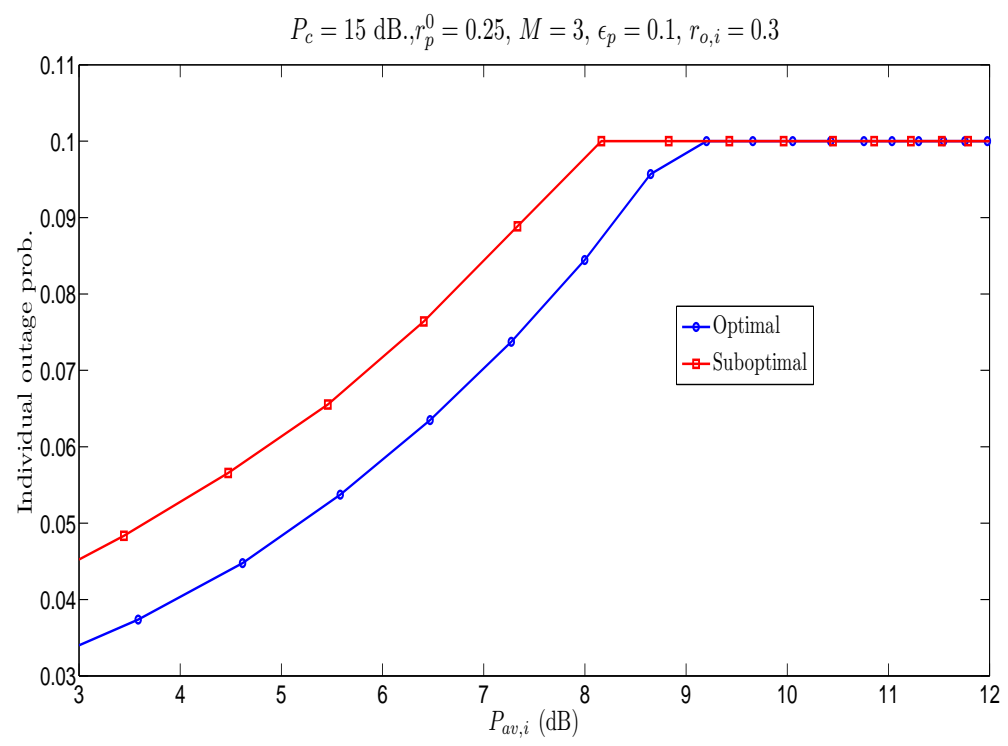

(b) Actual PU's outage probability performance

Figure 4.9: Effects of optimal and suboptimal decoding strategies on the SU individual outage probability and actual PU's outage probability: $P_{c}=15 \mathrm{~dB} ., M=3$, $r_{p}^{0}=0.25, r_{o, i}=0.4$ 
decoding is optimal due to the polymatroid structure of the problem. Numerical results were presented to illustrate the effect of the primary user's outage probability constraint on the achievable SU ergodic sum capacity and both common and individual outage probability regions. 


\section{Chapter 5}

\section{Outage Minimization under Primary Outage Constraint with Quantized Feedback}

In this chapter, we focus on the underlay paradigm where SUs can simultaneously transmit in the same frequency band with PUs, while ensuring the QoS level in the primary network. Optimization problems in the spectrum-sharing scheme draw wide attention from researchers since the performance in the primary network must be protected while SUs aim to maximize their performance with their limited resources, e.g. transmit power constraint. For instance, [39] studied SU outage probability minimization problem subject to average/peak transmit power constraint and average/peak interference power constraint on the primary receiver. Then, [44] investigated SU outage probability minimizing problem but considered the primary outage constraint (POC) instead of interference power constraint to ensure the QoS in delay-sensitive PU. Recently, [76] solved the service-outage maximizing problem for SU with POC and average transmit power constraint (ATPC).

However, the aforementioned literatures $[39,44,76]$ assume that SUs have perfect channel side information at transmitters (CSIT), although the CSIT is imperfect in a practical situation. The imperfect CSIT problem is more challenging in wireless communication research: $[59,64,68,86]$ for example studied various types of partial CSIT in a non-cognitive network. [59] considered the outage minimization problem for MIMO system with quantized feedback, i.e. the transmitter will receive $B$-bit feedback side information from the receiver or the band controller, which holds the channel knowledge information of the entire network. It is worth noting that [59] serves as a benchmark for our problem as we add POC to this problem in singleantenna case.

Nevertheless, the study of partial CSI for cognitive radio scenario remains an 
uncharted area. Motivated by this fact, [87] investigated the effect of partial CSI at the secondary transmitter on the ergodic capacity and outage capacity under average/peak intereference power constraint where the secondary transmitter has a noisy estimate of the true CSI. Then, the effect of imperfect channel estimation between a secondary transmitter and a primary receiver on the mean SU's channel capacity with impact of quantization levels on the CSI under peak transmit power and peak interference power constraints was studied in [88]. Recently, [71] offered a practical design of both cooperative feedback and feedforward between primary receiver and multi-antenna secondary transmitter and analyzed SU outage probability behavior accordingly.

This chapter aims to solve SU outage minimizing problem subject to POC and ATPC with quantized feedback. Under the same assumption as [60] and [61], secondary transmitter receives $B$-bit CSI through a perfect feedback link from a band controller who knows the entire channel power gains involved in the network. [60] addressed OFDM-based SU ergodic capacity maximization problem subject to an ATPC and $M$ average interference power constraints (AIPC) with quantized information of the vector channel space involving channel gains over all $M$ bands. In [61], the authors studied the SU outage minimization subject to ATPC and AIPC with a single pair of single-antenna PU and single-antenna SU and proposed low-complexity algorithm for suboptimal solution to the problem. They also proved the optimality of an $L$-region 'stepwise' channel partition structure where each region corresponds to the quantized power used in the channel state.

This chapter extends the problem in [44] to the $B$-bit feedback case, thus making it non-convex. However, standard Lagrange multiplier-based optimization technique can be used and a local optimal solution can be found via Karush-Kuhn-Tucker (KKT) necessary conditions. It is worth noting that the structure of this work follows that of [61] which considered outage minimization with limited feedback in cognitive scenario using KKT condition and proposed an efficient suboptimal algorithm. However, our work is different from [61] in three ways. Firstly, we consider POC which is more appropriate to ensure QoS for a delay-sensitive PU than AIPC in [61]. Secondly, except the asymptotic analysis on SU outage probability 
part, there is no any assumption on fading distributions of all channel power gains, making results in this chapter more general. Thirdly, our work also considers the practical effect of the interference channel gain from primary transmitter (PT) to secondary receiver (SR) as opposed to [61] and previous works in [38,39] which presume that the interference noise from primary transmitter to secondary receiver is negligible.

The contributions for this chapter are as follows: The optimal channel partition structure (CPS) for a given power codebook is obtained. Then, assuming that the PU transmits at constant power, we can use the optimal CPS to find a locally optimal power codebook through KKT condition, leading to the corresponding algorithm. Regardless of the type of continuous fading distribution of the channel gains, we later prove two important properties of the optimal codebook. Firstly, we show that the lowest power level approaches zero. Secondly, the ratio between two adjacent power levels is constant. By the first property, we propose a suboptimal solution by assuming that the lowest power level is zero, named 'Zero-Forced Lowest Power Level (ZFLP)', which is considered as more computationally efficient than the optimal solution and whose performance is close to the locally optimal algorithm when the number of feedback bits $B \geq 6$, as corroborated by our simulation results. Finally, if all channel gains are assumed to be Rayleigh fading, SU outage behaviour approximation from that suboptimal algorithm is examined when the number of bits is large. The simulation results suggest that the approximation is very close to the actual SU outage probability when $B \geq 8$ and the achievable SU outage probability performance is close to full CSI case when $B \geq 10$.

The rest of this chapter is organized as follows. Section 5.1 describes the system model and problem statement. In Section 5.2, we examine the problem in limited feedback scenario, derive the optimal channel partition structure (CPS) and use the KKT condition to solve for a (locally) optimal solution. We also show that the lowest power level converges to zero when the number of feedback bits is large, leading to a low-complexity ZFLP algorithm for the suboptimal solution as we force the lowest power level to be zero. Later, we analyze the outage behavior for SU when $B$ is large enough in Section 5.3, prior to presenting the numerical results in Section 5.4 
and ending the chapter by concluding remarks in Section 5.5.

\subsection{Problem statement and system model}

We consider a single primary transmitter-receiver pair (PT-PR) and a single secondary transmitter-receiver pair (ST-SR) accessing the same frequency band in a typical spectrum sharing scenario. All channels involved in this cognitive radio network are assumed to be independent BF-AWGN channels [21]. The additive noises at the PR and SR are assumed to be independent Gaussian random variables with zero mean and variance $N_{0}$. As shown in Figure 5.1, the instantaneous channel power gains for the link PT-PR, ST-SR, PT-SR, and ST-PR are denoted by $g, h, \alpha$, and $\beta$, respectively. Let $\nu=\{g, h, \alpha, \beta\}$ denote the combined channel state vector, presumed to be a vector of continuous random variables. The vector fading process $\nu$ is assumed to be stationary and ergodic. We assume that the delay-sensitive PU

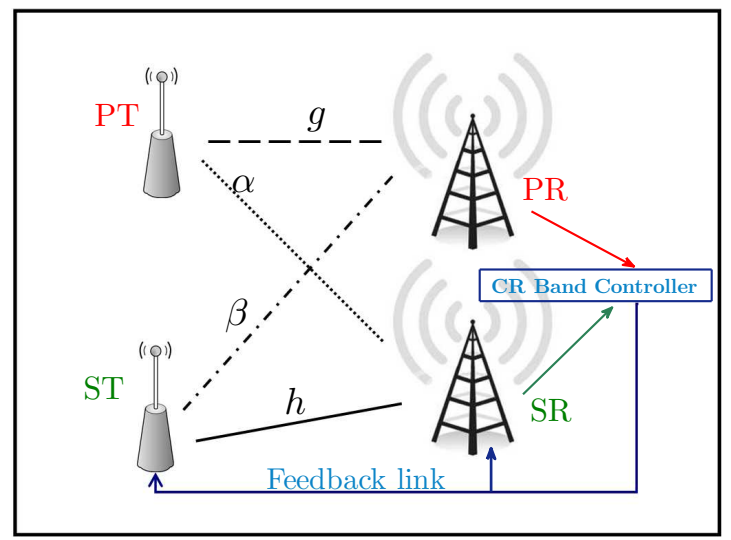

Figure 5.1: System Model in Chapter 5

uses constant power control denoted by $P_{c}$ and the SU adjusts its power, $p_{s}(\hat{\nu})$, according to the side information at secondary transmitter $\hat{\nu}$. Note that $\hat{\nu}=\nu$, if SU has full CSI for the entire network. Therefore, the rate expressions for both PU and SU can be defined as follows

$$
\begin{array}{ll}
r_{p}\left(\nu, p_{s}(\hat{\nu})\right)= & \log \left(1+\frac{g P_{c}}{\beta p_{s}(\hat{\nu})+N_{0}}\right) \\
r_{s}\left(\nu, p_{s}(\hat{\nu})\right)= & \log \left(1+\frac{h}{\alpha P_{c}+N_{0}} p_{s}(\hat{\nu})\right)
\end{array}
$$


Let the target rate and outage probability threshold of the PU communication be $r_{p}^{0}$ and $\epsilon_{p}$, respectively. As PU utilizes constant power control, PU will face outage with probability $\epsilon_{p}^{0}$, even if $\mathrm{SU}$ is not active: this occurs whenever $g<g_{T}=\frac{\left(\exp \left(r_{p}^{0}\right)-1\right) N_{0}}{P_{c}}$. Assuming that $\epsilon_{p} \geq \epsilon_{p}^{0}$, we will consider SU outage minimization problem subject to average transmit power constraint (ATPC) at SU and a primary user's outage probability constraint (POC) in order to protect the delay-sensitive PU, defined as follows

$$
\begin{array}{ll}
\min _{p_{s}(\hat{\nu})} & \operatorname{Pr}\left\{r_{s}\left(\nu, p_{s}(\hat{\nu})\right)<r_{s}^{0}\right\} \\
\text { s.t. } & \operatorname{Pr}\left\{r_{p}\left(\nu, p_{s}(\hat{\nu})\right)<r_{p}^{0}\right\} \leq \epsilon_{p} \\
& E\left[p_{s}(\hat{\nu})\right] \leq P_{a v} \\
& p_{s}(\hat{\nu}) \geq 0
\end{array}
$$

where $r_{s}^{0}$ denotes SU target rate. As illustrated in Figure 5.1, we assume that there is a cognitive-radio band controller (CR band controller) who can collect CSI of the entire network $\nu$ from PR and SR possibly from wireline connections, map the obtained CSI into quantized regions and feed back the quantized information, $\hat{\nu}$, via a perfect feedback link to ST using $B$ bits. Note that when $\hat{\nu}=\nu$, i.e. full CSI case, the optimal solution is proved to be truncated channel inversion proposed in [44] which is considered as a benchmark to our problem.

\subsection{SU outage minimization problem with quan- tized feedback}

In this section, we consider the limited-feedback case in which the $\mathrm{ST}$ receives $B$ feedback bits from the band controller, the ST will transmit power corresponding to that quantized feedback information $\hat{\nu}$. With $B$-bit feedback, we can have a power codebook $\mathbf{P}=\left[p_{1}, \ldots, p_{L}\right]$, where $L=2^{B}$. Without loss of generality, let $p_{1}>\ldots>p_{L}$. SU will utilize $p_{j}$ if $\hat{\nu} \in \mathcal{R}_{j}$. We first define the indicator function, $1(X)$, which is equal to 1 if the event $X$ is true and it is 0 otherwise. For convenience, 
we define variable $x$ and $\Gamma$ as follows:

$$
\begin{aligned}
x & =\frac{1}{\beta}\left(\frac{g P_{c}}{\exp \left(r_{p}^{0}\right)-1}-N_{0}\right) \\
\Gamma & =\frac{h}{\alpha P_{c}+N_{0}},
\end{aligned}
$$

where $c_{S U}=\exp \left(r_{s}^{0}\right)-1$.

It is easy to verify that the SU will face outage if it transmits with power $p_{j}$ such that $p_{j}<\frac{c_{S U}}{\Gamma}$, whereas the PU will be in outage if $p_{j}>x$. Therefore, we can reformulate the problem as follows

$$
\begin{array}{ll}
\min _{p_{j}, \mathcal{R}_{j}} & \sum_{j=1}^{L} \operatorname{Pr}\left(\mathcal{R}_{j}\right) E\left[1\left(p_{j}<\frac{c_{S U}}{\Gamma}\right) \mid \mathcal{R}_{j}\right] \\
\text { s.t. } & \sum_{j=1}^{L} \operatorname{Pr}\left(\mathcal{R}_{j}\right) E\left[1\left(p_{j}>x\right) \mid \mathcal{R}_{j}\right] \leq \epsilon_{p} \\
& \sum_{j=1}^{L} \operatorname{Pr}\left(\mathcal{R}_{j}\right) E\left[p_{j} \mid \mathcal{R}_{j}\right] \leq P_{a v} \\
& p_{j} \geq 0, \quad \forall j .
\end{array}
$$

Note that the problem (5.4) is nonconvex in general. Still, we can find a locally optimal solution by using standard Lagrange multiplier-based optimization and then applying Karush-Kuhn-Tucker (KKT) necessary condition. Hence, we first write the Lagrangian corresponding to (5.4) as follows:

$$
L\left(\lambda, s, p_{j}, \mathcal{R}_{i}\right)=\left(\sum_{j=1}^{L} \operatorname{Pr}\left(\mathcal{R}_{j}\right)\left[1\left(p_{j}<\frac{c_{S U}}{\Gamma}\right)+s 1\left(p_{j}>x\right)+\lambda p_{j} \mid \mathcal{R}_{j}\right]\right)-s \epsilon_{p}-\lambda P_{a v}
$$

where $s$ and $\lambda$ are the Lagrange multipliers for POC and ATPC, respectively. Then, the dual problem can be written as

$$
\max _{\lambda, s}\left[\min _{p_{j} \geq 0, \mathcal{R}_{j}} L\left(\lambda, s, p_{j}, \mathcal{R}_{i}\right)\right]
$$

In (5.5), one can notice that there is no obvious way to directly obtain a gradient of the Lagrangian with respect to $p_{j}$. However, a local optimum for (5.6) can be solved by using the Simultaneous Perturbation Stochastic Approximation (SPSA) 
algorithm [89] with subgradient method [90]. However, it is worth mentioning that, the SPSA approach returns a locally optimal solution and there is no explicit expression for both SU and PU outage probability, leading to very high computational complexity and thus a very long convergence time. To reduce the complexity in searching for optimal codebook and quantized channel partition region, we will first derive the optimal channel partition structure (CPS) as a function of given power codebook $\mathbf{P}$. The optimal channel partition structure will help us express both SU and PU outage probability functions in term of the power codebook, allowing us to utilize the KKT condition to solve for a local optimum, but with faster computation time compared to SPSA.

\subsubsection{Optimal channel partition structure and searching al- gorithm}

The optimal channel partition structure (CPS) for a given codebook $\mathbf{P}$ can be defined as follows.

Lemma 5.2.1. (Channel Partition Structure) For given $\lambda$, s and power codebook $\mathbf{P}$, the power control can be defined as follows.

$$
p(\Gamma, x)= \begin{cases}p_{j}, & 1 \leq j \leq k, x \notin\left(p_{L}, p_{j}\right], \Gamma \in\left[\frac{c_{S U}}{p_{j}}, \frac{c_{S U}}{p_{j+1}}\right) \\ p_{j}, & k+1 \leq j \leq L, \Gamma \in\left[\frac{c_{S U}}{p_{j}}, \frac{c_{S U}}{p_{j+1}}\right) \\ p_{L}, & \text { otherwise }\end{cases}
$$

where $k=\arg \min _{j: p_{j}>p_{L}+\frac{1-s}{\lambda}} p_{j}$ and $k$ becomes 0 when $s=0$.

Proof. Please refer to Appendix D.1.

It is worth noting that our CPS in Lemma 5.2.1 becomes similar to previous result in [59] when $s=0$, i.e. when POC is inactive. By Lemma 5.2.1, CPS can be illustrated in Figure 5.2 regardless of the channel power gains involved in this problem. Note that the grey line separates the outage and non-outage region of PU, i.e. $\mathrm{PU}$ is in outage for channel state on the left-hand side of this line, while PU does not face an outage when channel state lies in the right-hand side of the line. 


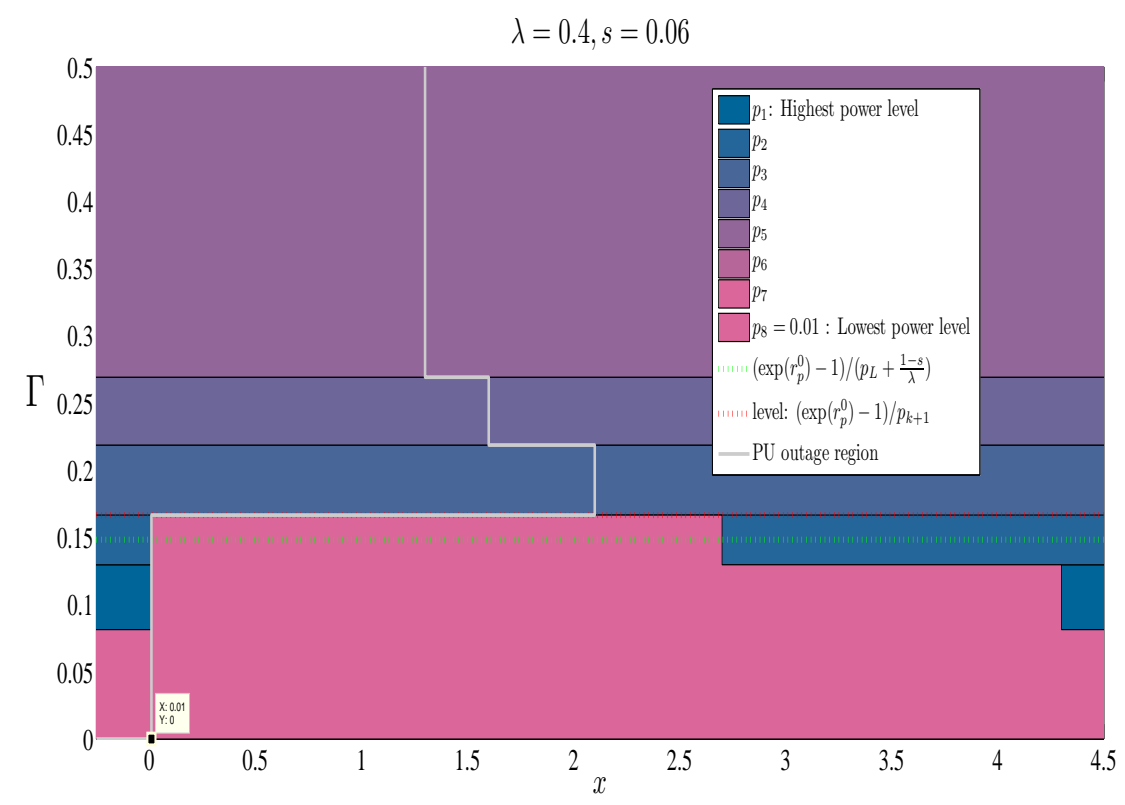

Figure 5.2: Channel partition structure with power levels for outage minimization problem with quantized CSI case $(B=3)$

Let $F_{G}(g)$ and $F_{\Gamma}(\Gamma)$ denote the CDF of $g$ and $\Gamma$. Given that $g \geq g_{T}$, CDF of $\tilde{X}$ is represented by $F_{\tilde{X}}(x)$, i.e. $F_{\tilde{X}}(x)=F_{X}\left(x \mid g \geq g_{T}\right)$. Also, let $\tau_{G}=1-F_{G}\left(g_{T}\right)$. Thus, (5.6) can be transformed to

$$
\max _{\lambda, s}\left[\min _{p_{j} \geq 0} G_{O}+s G_{1}+\lambda G_{2}\right]
$$

where $G_{O}, G_{1}$ and $G_{2}$ is expressed by

$$
\begin{aligned}
G_{O}= & F_{\Gamma}\left(\frac{c_{S U}}{p_{1}}\right)+\tau_{G} \sum_{j=1}^{k}\left[F_{\tilde{X}}\left(p_{j}\right)-F_{\tilde{X}}\left(p_{L}\right)\right]\left(F_{\Gamma}\left(\frac{c_{S U}}{p_{j+1}}\right)-F_{\Gamma}\left(\frac{c_{S U}}{p_{j}}\right)\right) \\
G_{1}= & \left(1-\tau_{G}\right)+\tau_{G} F_{\tilde{X}}\left(p_{L}\right)+\tau_{G} \sum_{j=k+1}^{L-1}\left[F_{\tilde{X}}\left(p_{j}\right)-F_{\tilde{X}}\left(p_{L}\right)\right]\left(F_{\Gamma}\left(\frac{c_{S U}}{p_{j+1}}\right)-F_{\Gamma}\left(\frac{c_{S U}}{p_{j}}\right)\right) \\
G_{2}= & p_{L}\left(1-F_{\Gamma}\left(\frac{c_{S U}}{p_{L}}\right)+F_{\Gamma}\left(\frac{c_{S U}}{p_{1}}\right)\right)+\tau_{G} \sum_{j=1}^{k}\left(p_{L}-p_{j}\right)\left(F_{\Gamma}\left(\frac{c_{S U}}{p_{j+1}}\right)-F_{\Gamma}\left(\frac{c_{S U}}{p_{j}}\right)\right)\left[F_{\tilde{X}}\left(p_{j}\right)-F_{\tilde{X}}\left(p_{L}\right)\right] \\
& +\sum_{j=1}^{L-1} p_{j}\left(F_{\Gamma}\left(\frac{c_{S U}}{p_{j+1}}\right)-F_{\Gamma}\left(\frac{c_{S U}}{p_{j}}\right)\right)
\end{aligned}
$$

Note that $G_{O}$ describes SU's outage probability, $G_{1}$ describes PU's outage probability and $G_{2}$ describes SU's power usage. 


\subsubsection{Locally optimal power codebook from KKT condition}

The power codebook $\mathbf{P}$ must satisfy KKT necessary condition for a local optimum, i.e. $\frac{\partial}{\partial p_{j}} L(\ldots)=0$ for all $j$. Let $F_{X}^{\prime}(x)$ denotes the first-order derivative with respect to $x$.

$$
\begin{aligned}
& \text { For } j=1 \\
& \begin{aligned}
0 & =-\frac{c_{S U}}{p_{1}^{2}} F_{\Gamma}^{\prime}\left(\frac{c_{S U}}{p_{1}}\right)+\tau_{G}\left(\frac{c_{S U}}{p_{1}^{2}} F_{\Gamma}^{\prime}\left(\frac{c_{S U}}{p_{1}}\right)\left[F_{\tilde{X}}\left(p_{1}\right)-F_{\tilde{X}}\left(p_{2}\right)\right]+F_{\tilde{X}}^{\prime}\left(p_{1}\right)\left[F_{\Gamma}\left(\frac{c_{S U}}{p_{2}}\right)-F_{\Gamma}\left(\frac{c_{S U}}{p_{1}}\right)\right]\right) \\
& +\lambda\left\{\left[F_{\Gamma}\left(\frac{c_{S U}}{p_{2}}\right)-F_{\Gamma}\left(\frac{c_{S U}}{p_{1}}\right)\right]-\frac{c_{S U}}{p_{1}^{2}} p_{L} F_{\Gamma}^{\prime}\left(\frac{c_{S U}}{p_{1}}\right)+\tau_{G}\left(p_{1}-p_{2}\right)\left[F_{\tilde{X}}\left(p_{1}\right)-F_{\tilde{X}}\left(p_{L}\right)\right] \frac{c_{S U}}{p_{1}^{2}} F_{\Gamma}^{\prime}\left(\frac{c_{S U}}{p_{1}}\right)\right\} \\
& -\lambda \tau_{G}\left\{\left(\left[F_{\Gamma}\left(\frac{c_{S U}}{p_{2}}\right)-F_{\Gamma}\left(\frac{c_{S U}}{p_{1}}\right)\right]\left[F_{\tilde{X}}\left(p_{1}\right)-F_{\tilde{X}}\left(p_{2}\right)\right]+\left(p_{1}-p_{2}\right)\left[F_{\Gamma}\left(\frac{c_{S U}}{p_{2}}\right)-F_{\Gamma}\left(\frac{c_{S U}}{p_{1}}\right)\right] F_{\tilde{X}}^{\prime}\left(p_{1}\right)\right)\right\}
\end{aligned}
\end{aligned}
$$

Thus, we can write $p_{2}$ as a function of $p_{1}$ and $p_{L}$ by solving $\frac{\partial L}{\partial p_{1}}$. For $1<j<L, p_{j+1}$ can be expressed as a function of $p_{j-1}, p_{j}$ and $p_{L}$ by solving $\frac{\partial L}{\partial p_{j}}$. The expressions of $\frac{\partial L}{\partial p_{j}}$ for all $j$ can be found in Appendix D.2. By using these conditions, the algorithm to search for a locally optimal codebook is summarized below.

\section{Two steps for solving the optimization problem}

1. For fixed values $\lambda$ and $s$, there are $L$ equations from $\frac{\partial L}{\partial p_{j}}=0$ that need to be satisfied by the locally optimal power codebook. Given $p_{1}$ and $p_{L}, p_{2}, \ldots, p_{L-1}$ can be computed successively from $\frac{\partial L}{\partial p_{j}}=0$ for $j=1, \ldots, L-2$. Then, we can numerically solve $p_{1}$ and $p_{L}$ such that $\frac{\partial L}{\partial p_{L-1}}=0$ and $\frac{\partial L}{\partial p_{L}}=0$ are satisfied. We can implement the numerical computation in MATLAB by using function fzero, for example.

2. To update the optimal value $\lambda$ and $s$, the subgradient method [90] can be used by the following equations.

$$
\begin{aligned}
& s_{m+1}=\left[s_{m}-\vartheta_{m}\left(\epsilon_{p}-G_{1}\right)\right]^{+} \\
& \lambda_{m+1}=\left[\lambda_{m}-\varsigma_{m}\left(P_{a v}-G_{2}\right)\right]^{+}
\end{aligned}
$$

where $m$ is the iteration number. For the $m$-th iteration, $\vartheta_{m}$ and $\varsigma_{m}$ represent the step sizes which satisfy square summable, but not summable step size, i.e. $\sum_{m=1}^{\infty} \vartheta_{m}^{2}<\infty$ and $\sum_{m=1}^{\infty} \vartheta_{m}=\infty$ and likewise for $\varsigma_{m}$ 
Remark 5.2.1. For a given $P_{a v}$ and $\epsilon_{p}$, one can first assume that $s=0$, i.e. POC is inactive. Next, apply the two steps above to solve for $p_{1}, \ldots, p_{L}$ and $\lambda$. Then, check whether the returned optimal solution satisfies POC or not. If it does, this is the optimal solution. Otherwise, $s>0$ must also be determined.

For the locally optimal solution, it is noticeable that, for given $\lambda_{m}$ and $s_{m}, p_{1}$ and $p_{L}$ must be initialized in order to compute $p_{2}, \ldots, p_{L-1}$ and then these initialized $p_{1}$ and $p_{L}$ must be checked via KKT condition. Hence, although it returns a locally optimal solution, the algorithm is tedious. To alleviate the problem, we seek another efficient suboptimal solution which performs very close to the local optimal one. Our suboptimal approach adopts the idea from the optimal approach when the number of bits $B$ is large. The properties are summarized in the following subsection.

\subsubsection{Proposed suboptimal algorithm}

In this part, we will start from Lemma 5.2.2 which analyzes the behavior of the lowest power level of the locally optimal codebook as the number of bits grows large. Then, we will use the intuition from Lemma 5.2.2 for our proposed suboptimal algorithm.

Lemma 5.2.2. $\lim _{L \rightarrow \infty} p_{L}=0$.

Proof. Please refer to Appendix D.3.

Noticeably, $L$-th power level $p_{L}$ appears in all the KKT conditions. With a large number of bits, repeatedly initializing both $p_{1}$ and $p_{L}$ makes the algorithm described in Section 5.2.2 tedious. Lemma 5.2.2 suggests that as the number of bits gets larger, $p_{L}$ will approach zero. By fixing $p_{L}=0$, the KKT conditions and searching algorithm become less complicated, although they no longer return a locally optimal solution to the original problem. The obtained algorithm is described below. In our simulation results, we will compare it to another suboptimal algorithm based on the generalized Lloyd's algorithm with Sigmoid Function Approximation (GLA+SFA) from [70] and described briefly in Appendix D.4

Zero-Forced Lowest Power Level Algorithm (ZFLP) 
In the algorithm for the locally optimal solution in Section 5.2.2, we have to initialize both $p_{1}$ and $p_{L}$. As noted earlier, the algorithm will be very slow if the number of bits is large. Hence, rather than initializing both $p_{1}$ and $p_{L}$, ZFLP algorithm assumes that $p_{L}$ is zero. The rationale behind this suboptimal algorithm is from Lemma 5.2.2 as explained previously. This algorithm therefore reduces the complexity in searching computation.

By assuming that $p_{L}=0$, we have $F_{\Gamma}\left(\frac{c_{S U}}{p_{L}}\right)=F_{\Gamma}(\infty)=1$ and $F_{\tilde{X}}\left(p_{L}\right)=0$. We can rewrite $(5.8)$ as

$$
\max _{\lambda, s}\left[\min _{p_{j} \geq 0} G_{O, s}+s G_{1, s}+\lambda G_{2, s}\right]
$$

where $G_{O, s}, G_{1, s}$ and $G_{2, s}$ is expressed by

$$
\begin{aligned}
G_{O, s} & =F_{\Gamma}\left(\frac{c_{S U}}{p_{1}}\right)+\tau_{G} \sum_{j=1}^{k}\left[F_{\tilde{X}}\left(p_{j}\right)\right]\left(F_{\Gamma}\left(\frac{c_{S U}}{p_{j+1}}\right)-F_{\Gamma}\left(\frac{c_{S U}}{p_{j}}\right)\right) \\
G_{1, s} & =\left(1-\tau_{G}\right)+\tau_{G} \sum_{j=k+1}^{L-1} F_{\tilde{X}}\left(p_{j}\right)\left(F_{\Gamma}\left(\frac{c_{S U}}{p_{j+1}}\right)-F_{\Gamma}\left(\frac{c_{S U}}{p_{j}}\right)\right) \\
G_{2, s}= & \sum_{j=1}^{L-1} p_{j}\left(F_{\Gamma}\left(\frac{c_{S U}}{p_{j+1}}\right)-F_{\Gamma}\left(\frac{c_{S U}}{p_{j}}\right)\right)-\tau_{G} \sum_{j=1}^{k} p_{j} F_{\tilde{X}}\left(p_{j}\right)\left(F_{\Gamma}\left(\frac{c_{S U}}{p_{j+1}}\right)-F_{\Gamma}\left(\frac{c_{S U}}{p_{j}}\right)\right)
\end{aligned}
$$

Based on the KKT condition, we must have $\frac{\partial L(\ldots)}{\partial p_{j}}=0$ for all $j$. For example, when $j=1$, it yields

$$
\begin{aligned}
& 0=-\frac{c_{S U}}{p_{1}^{2}}\left(1-\tau_{G} F_{\tilde{X}}\left(p_{1}\right)\right) F_{\Gamma}^{\prime}\left(\frac{c_{S U}}{p_{1}}\right)+\tau_{G}\left(F_{\Gamma}\left(\frac{c_{S U}}{p_{2}}\right)-F_{\Gamma}\left(\frac{c_{S U}}{p_{1}}\right)\right) F_{\tilde{X}}^{\prime}\left(p_{1}\right) \\
& +\lambda\left[\left(F_{\Gamma}\left(\frac{c_{S U}}{p_{2}}\right)-F_{\Gamma}\left(\frac{c_{S U}}{p_{1}}\right)\right)\left[1-\tau_{G} F_{\tilde{X}}\left(p_{1}\right)-p_{1} \tau_{G} F_{\tilde{X}}^{\prime}\left(p_{1}\right)\right]\right. \\
& \left.\quad+p_{1}\left(1-\tau_{G} F_{\tilde{X}}\left(p_{1}\right)\right) \cdot \frac{c_{S U}}{p_{1}^{2}} F_{\Gamma}^{\prime}\left(\frac{c_{S U}}{p_{1}}\right)\right]
\end{aligned}
$$

This suggests that $p_{2}$ can be written as a function of $p_{1}$. Likewise, for $1<j<L$ (details in Appendix D.5), we can show that $p_{j+1}$ can be determined from a given $p_{j}$ through the condition $\frac{\partial L(\ldots)}{\partial p_{j}}=0$. By those facts, for given $\lambda$ and $s$, we first initialize the value of $p_{1}$ so that $p_{2}, \ldots, p_{L-1}$ can be recursively computed in term of $p_{1}$ by using the conditions $\frac{\partial L(\ldots)}{\partial p_{j}}=0$ for $j=1, \ldots, L-2$. At the end, the initialized $p_{1}$ must ensure that the condition $\frac{\partial L(\ldots)}{\partial p_{L-1}}=0$ is satisfied. Then, we can use subgradient method, as shown previously in (5.10), to update for $\lambda$ and $s$. The procedures are repeated until convergence. 


\subsection{Asymptotic analysis on SU outage probability for ZFLP}

In this section, we aim at analyzing the asymptotic behavior of SU outage probability as $L$ grows large, when all channel power gains are presumed to be exponentially distributed, i.e. under a Rayleigh fading assumption.

Lemma 5.3.1. Regardless of the fading distributions of channel gains, we have $\frac{p_{j}}{p_{j+1}}=\frac{p_{j-1}}{p_{j}}$ for $1<j<k+1$ and $k+1<j<L-1$ as $L \rightarrow \infty$.

Proof. Please refer to Appendix D.6

In this part, all channel power gains are further presumed to be exponentially distributed, so $F_{\Gamma}(\Gamma)=\left(1-\frac{\exp \left(-N_{0} \Gamma\right)}{1+P_{c} \Gamma}\right), F_{\tilde{X}}(x)=1-\frac{1}{1+c_{o} x}$, where $c_{o}=\frac{\exp \left(r_{p}^{0}\right)-1}{P_{c}}$ and $\tau_{G}=e^{-g_{T}}$. We will use the expressions on those CDF to analyze SU outage probability when $L$ is large. According to Lemma 5.2.2, we can assume that $p_{L}=0$. Also, $\lambda \approx \lambda_{f}$ and $s \approx s_{f}$, where $\lambda_{f}$ and $s_{f}$ are the Lagrange multipliers corresponding to ATPC and POC in the full CSI case. From Lemma 5.3.1, let $\frac{p_{j}}{p_{j+1}} \approx \frac{p_{j-1}}{p_{j}}=\theta$ for $1 \leq j \leq k, \frac{p_{j}}{p_{j+1}} \approx \frac{p_{j-1}}{p_{j}}=\kappa$ for $k+1 \leq j \leq L-1$. For simplicity, define $f_{1}\left(p_{j}\right)$ and $f_{2}\left(p_{j}\right)$ as follows

$$
\begin{aligned}
f_{1}\left(p_{j}\right) & =\lambda p_{j}\left(1-e^{-g_{T}} F_{\tilde{X}}\left(p_{j}\right)\right)+e^{-g_{T}} F_{\tilde{X}}\left(p_{j}\right) \\
& =\lambda p_{j}\left(1-e^{-g_{T}}\left[1-\frac{1}{1+c_{o} p_{j}}\right]\right)+e^{-g_{T}}\left[1-\frac{1}{1+c_{o} p_{j}}\right] \\
f_{2}\left(p_{j}\right) & =s e^{-g_{T}} F_{\tilde{X}}\left(p_{j}\right)+\lambda p_{j}=s e^{-g_{T}}\left[1-\frac{1}{1+c_{o} p_{j}}\right]+\lambda p_{j}
\end{aligned}
$$

Thus, $f_{1}^{\prime}\left(p_{j}\right)=\lambda\left(1-e^{-g_{T}}\left[1-\frac{1}{1+c_{o} p_{j}}\right]\right)+e^{-g_{T}} \frac{c_{o}\left(1-\lambda p_{j}\right)}{\left(1+c_{o} p_{j}\right)^{2}}$ and $f_{2}^{\prime}\left(p_{j}\right)=s e^{-g_{T}} \frac{1}{\left(1+c_{o} p_{j}\right)^{2}}+$ $\lambda$. Let $v_{j}=\frac{c_{S U}}{p_{j}}$. Then, we can prove the two following lemmas.

Lemma 5.3.2. As L grows large, $\theta$ can be approximated from solving

$$
\theta=\left[\frac{1-f_{1}\left(\frac{p_{0}}{\theta}\right)+\frac{p_{0}}{\theta} f_{1}^{\prime}\left(\frac{p_{0}}{\theta}\right)}{\frac{p_{0}}{\theta} f_{1}^{\prime}\left(\frac{p_{0}}{\theta}\right)}\right]
$$

where $p_{0} \triangleq \frac{1}{\lambda}$.

Proof. Please Refer to Appendix D.7 
Lemma 5.3.3. As L grows large, we have $\kappa \approx \theta$.

Proof. Please refer to Appendix D.8

By Lemma 5.3.2 and 5.3.3, we can now write $p_{j}=p_{0} \theta^{-j}$ for all $j$. As $L$ is large, $p_{j} \approx\left(1-j \epsilon_{\theta}\right)$, making $0<\epsilon_{\theta}<\frac{1}{L-1}$ and $\lim _{L \rightarrow \infty} \theta=1$. Next, $\theta$ can be determined via using two following equations.

$$
\begin{aligned}
\epsilon_{p} & \left(1-e^{-g_{T}}\right)=e^{-g_{T}} \sum_{j=k+1}^{L-1} F_{\tilde{X}}\left(p_{j}\right)\left(F_{\Gamma}\left(\frac{c_{S U}}{p_{j+1}}\right)-F_{\Gamma}\left(\frac{c_{S U}}{p_{j}}\right)\right) \approx \mathcal{F}_{P O C}(\theta, k) \\
P_{a v} & =\sum_{j=1}^{L-1} p_{j}\left(F_{\Gamma}\left(\frac{c_{S U}}{p_{j+1}}\right)-F_{\Gamma}\left(\frac{c_{S U}}{p_{j}}\right)\right)-e^{-g_{T}} \sum_{j=1}^{k} p_{j} F_{\tilde{X}}\left(p_{j}\right)\left(F_{\Gamma}\left(\frac{c_{S U}}{p_{j+1}}\right)-F_{\Gamma}\left(\frac{c_{S U}}{p_{j}}\right)\right) \\
& \approx \mathcal{F}_{A T P C}(\theta, k)
\end{aligned}
$$

The expressions of $\mathcal{F}_{P O C}(\theta, k)$ and $\mathcal{F}_{A T P C}(\theta, k)$ are included in Appendix D.9. Further, we can approximate $k$ by using the fact that $\lim _{L \rightarrow \infty} k=k(\theta)=\left\lceil\frac{-\log (1-s)}{\log \theta}\right\rceil$. Hence, we can determine $\theta$ by solving

$$
s \epsilon_{p}-\left(1-e^{-g_{T}}\right)+\lambda P_{a v}=s \mathcal{F}_{P O C}(\theta, k(\theta))+\lambda \mathcal{F}_{A T P C}(\theta, k(\theta))
$$

For the objective function, we finally have

$$
\begin{aligned}
\epsilon_{s} & =F_{\Gamma}\left(v_{1}\right)+e^{-g_{T}} \sum_{j=1}^{k} F_{\tilde{X}}\left(p_{j}\right)\left[F_{\Gamma}\left(v_{j+1}\right)-F_{\Gamma}\left(v_{j}\right)\right] \\
& \stackrel{(d)}{\approx} 1-\frac{e^{-N_{0} v_{1}}}{1+P_{c} v_{1}}\left(1-\frac{P_{c} c_{o} c_{S U} e^{-g_{T}}}{P_{c} c_{o} c_{S U}-1}\right)-\frac{P_{c} c_{o} c_{S U} e^{-g_{T}}}{P_{c} c_{o} c_{S U}-1} \cdot \frac{P_{c} e^{-N_{0} v_{1} \theta^{k}}}{1+P_{c} v_{1} \theta^{k}}+A_{4}\left(v_{1} \theta^{k}\right)-A_{4}\left(v_{1}\right) \\
\lim _{L \rightarrow \infty} \epsilon_{S} & =1-\frac{e^{-N_{0} c \lambda}}{1+P_{c} c \lambda}\left(1-\frac{P_{c} c_{c} c_{S U} e^{-g} T}{P_{c} c_{o} c_{S U}-1}\right)-\frac{P_{c} c_{o} c_{S U} e^{-g_{T}}}{P_{c} c_{S} c_{S U}-1} \cdot \frac{P_{c} e^{-N_{0} \frac{c \lambda}{1-s}}}{1+P_{c} \frac{c \lambda}{1-s}}+A_{4}\left(\frac{c \lambda}{1-s}\right)-A_{4}(c \lambda)
\end{aligned}
$$

Again, the summation can be approximated by integration shown in (5.17) and $A_{4}(z)$ is expressed by

$$
\begin{aligned}
A_{4}(z) & =\frac{c_{o} c_{S U} e^{-g_{T}}}{P_{c} c_{o} c_{S U}-1}\left[\left(N_{0}-\frac{P_{c}}{P_{c} c_{o} c_{S U}-1}\right) e^{-g_{T}} e^{N_{0} c_{o} c_{S U}} E_{1}\left(N_{0}\left(z+c_{o} c_{S U}\right)\right)\right. \\
& \left.+\frac{P_{c} e^{\frac{N_{0}}{P_{c}}}}{P_{c} c_{o} c_{S U}-1} E_{1}\left(N_{0} z+\frac{N_{0}}{P_{c}}\right)\right]
\end{aligned}
$$

Remark 5.3.1. If $s=0, k$ becomes zero and $\theta$ is determined from average power constraint. Then, $\epsilon_{s} \approx 1-\frac{e^{-N_{0} v_{1}}}{1+P_{c} v_{1}}=1-\frac{e^{-N_{0} c \lambda \theta}}{1+P_{c} c \lambda \theta}$ and $\lim _{L \rightarrow \infty} \epsilon_{s}=1-\frac{e^{-N_{0} c \lambda}}{1+P_{c} c \lambda}$ equivalent 
to the result in $[61]$.

\subsection{Simulation results}

In this part, the numerical results on SU performance are illustrated for the SU outage minimizing problem subject to ATPC and POC based on various algorithms mentioned in this chapter. All channel gains involved are assumed to be independent and identically distributed (i.i.d.) and exponentially distributed (Rayleigh fading) with unit mean. Noises at PR and SR are presumed to be equal and AWGN with a normalized unit variance. This allows us to consider the SU and PU transmit powers and the SU average transmit power constraint in unitless terms, thus expressed in $\mathrm{dB}$. Note also that the SU average transmission power can be regarded as the average transmit SNR at the SU transmitter. Unless specified otherwise, the simulation parameters are set as: $\mathrm{SU}$ target rate $r_{s}^{0}=0.3, \mathrm{PU}$ constant power $P_{c}=15 \mathrm{~dB}$, $\mathrm{PU}$ target rate $r_{s}^{0}=0.2$ and $\mathrm{PU}$ outage probability threshold $\epsilon_{p}=0.1$.

Figure 5.3 compares SU outage performance of ZFLP algorithm for suboptimal solution with local optimal solution and another suboptimal solution GLA+SA with varying number of feedback bits $B=\{1,2\}$. SU outage performances when the secondary transmitter has no $\operatorname{CSIT}(B=0)$ and when it has full CSI $(B=\infty)$ are also included as a reference. First, the figure shows that SU outage probability performance from locally optimal solution is improved significantly (by about $41.5 \%$ ) with $B=1$ compared to the performance in no CSI case. It confirms the benefit of CSI feedback. Also, the results reveal that the SU outage probability of ZFLP algorithm surpasses that of GLA+SA for any given $B$. For example, with a fixed $P_{a v}=11.5 \mathrm{~dB}$, achieved outage probability from GLA+SA is $\epsilon_{s}=0.3457$ for $B=1$ and $\epsilon_{s}=0.2260$ for $B=2$, while ZFLP can achieve $\epsilon_{s}=0.2887$ for $B=1$ and $\epsilon_{s}=0.1941$ for $B=2$ which are approximately $16.5 \%$ and $20.17 \%$ lower than that of GLA+SA. Further, ZFLP is considerably faster than GLA+SA. With Intel(R) Core(TM)2 Duo CPU and 2.99GHz with 3.48GB RAM, GLA+SA took roughly 988.3412 seconds for $B=2$. (SFA procedure was done with $2 \times 10^{5}$ samples, starting from $K=20$ with the increment of $K$ is 1.5 , ending at $K=672.3$ when 
convergence is met. Note that the initial guess of power codebook may cause a different convergence time.) On the other hand, ZFLP took just 176.51 seconds to achieve the same level of accuracy. Thus, ZFLP is more efficient than GLA+SFA both from a performance and a time-consumption points of views.

Moreover, Figure 5.3 shows that, although ZFLP algorithm is for suboptimal solution, the SU outage probability performance from ZFLP approaches that of the optimal solution when $B$ increases. As mentioned earlier, one has to initialize both $p_{1}$ and $p_{L}$ to search for $p_{2}, \ldots, p_{L-1}$ and keep updating $p_{1}$ and $p_{L}$ till the constraints are satisfied for the optimal solution. The outage for $\mathrm{SU}$ is about 0.2577 for $1 \mathrm{bit}$ and 0.1749 for 2 bits feedback in optimal solution with $P_{a v}=11.5 \mathrm{~dB}$. The result then suggests that SU outage performance can be sacrificed for $12.0 \%$ when $B=1$ and $3.1 \%$ when $B=2$ by just assuming $p_{L}=0$ to ease time consuming problem. Note that the gap between ZFLP and the optimal are closer when $B$ is raised.

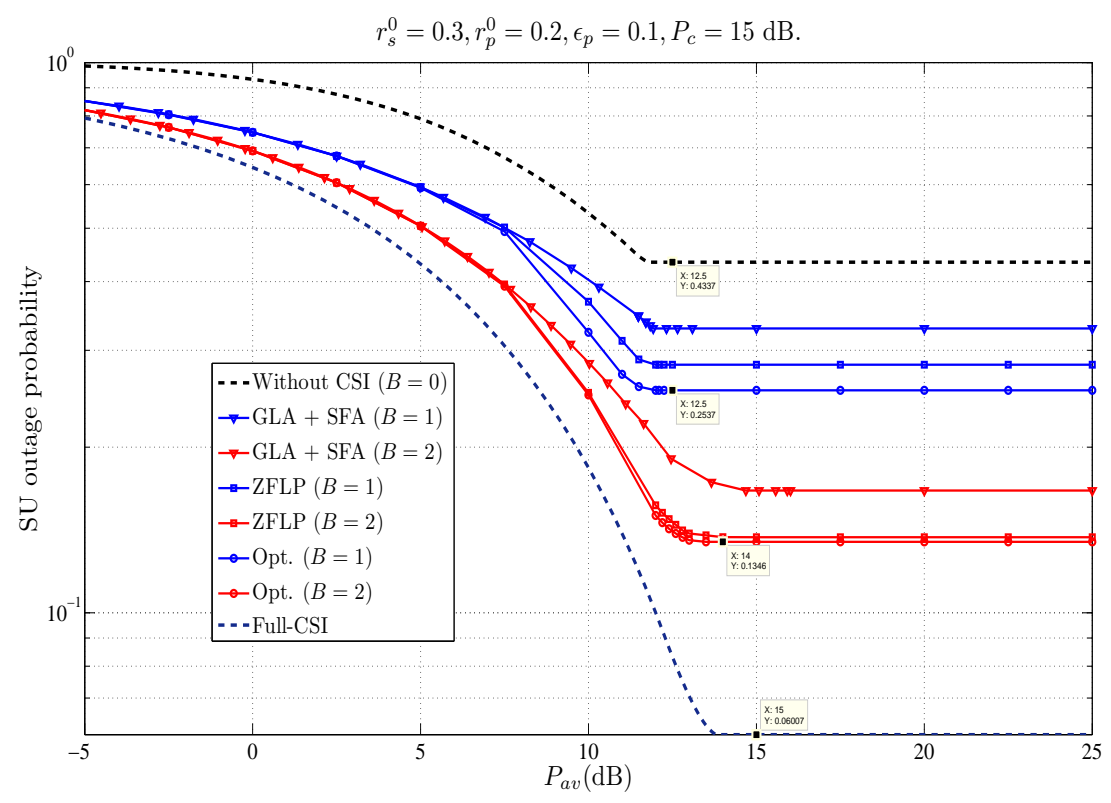

Figure 5.3: SU outage probability against average power $P_{a v}$ with varying number of feedback bits by the three algorithms

Next, the effect of $\mathrm{PU}$ target rate $r_{p}^{0}$ on $\mathrm{SU}$ outage probability performance for quantized feedback will be inspected. With $r_{p}^{0}=\{0.2,0.4\}$, we thus study the performance for three varying number of feedback bits, i.e. $B=\{4,6,10\}$ and for full CSI as a reference. There are four important results shown in Figure 5.4. Firstly, 
with the same $B$, SU outage probability when $r_{p}^{0}=r_{p, 2}^{0}=0.4$ always greater than that of when $r_{p}^{0}=r_{p, 1}^{0}=0.2$ obviously because POC in the first case is stricter. Secondly, under the same $r_{p}^{0}$, one additional feedback bit can reduce the gap of SU outage performance. However, it can be observed that the size of the reduced gap is smaller and smaller as $B$ approaches infinity, i.e. full CSI case. Thirdly, regardless the number of feedback bits and $r_{p}^{0}$, the feature of achieving SU outage probability is similar, i.e. SU outage drops significantly when $P_{a v}$ is low but reach saturation when $P_{a v}$ is high. Intuitively, it implies that, despite having abundant average power budget, SU cannot always enjoy using that as QoS in primary link must be protected. Lastly, SU outage performances when $B=6$ due to the optimal approach and ZFPL suboptimal approach are almost overlapped at $P_{a v}=11.5 \mathrm{~dB}$, the difference is about $6.6 \times 10^{-5}$ for $r_{p}^{0}=0.2$ where $\mathrm{SU}$ outage probability is around 0.1726 and about $7.3 \times 10^{-5}$ for $r_{p}^{0}=0.4$ where SU outage probability is around 0.1260. Thus, we avoid very high complexity in optimal approach by using ZFLP when $B=10$ and the results for both target rates shows that SU outage probability performance is very close to full CSI case when $B=10$.

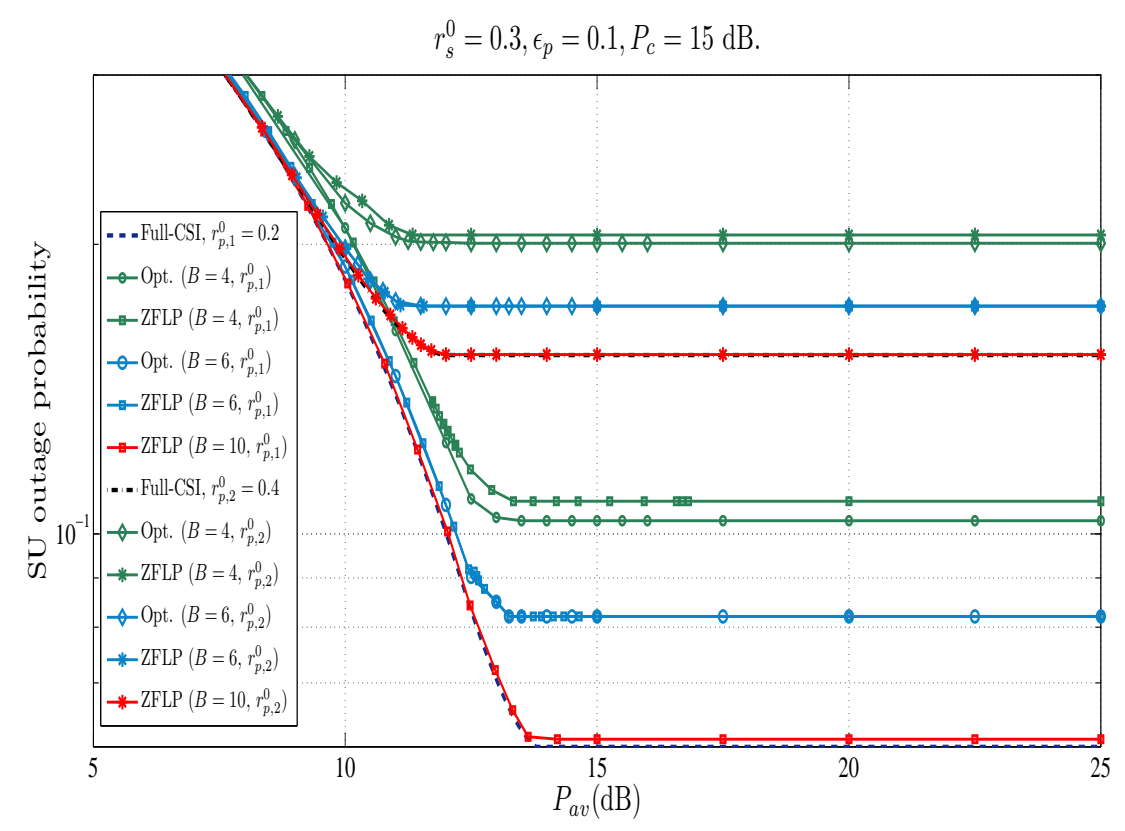

Figure 5.4: SU outage probability against average power $P_{a v}$ with varying $r_{p}^{0}$

In Figure 5.5, SU probability results based on ZFLP algorithm for $B=\{2,4,6,8\}$ are plotted against SU probability results from asymptotic analysis in Section 5.3. 
The result shows that the reduction in the gap between outage performance in ZFLP and its approximation. For example, at $P_{a v}=11.5 \mathrm{~dB}$. i.e. the difference is approximately $14.01 \%, 6.22 \%, 2.46 \%$ and $0.926 \%$ for $B=2,4,6,8$, respectively. This is as expected because the analysis in Section 5.3 is based on the assumption that $L$ approaches $\infty$, so the higher the $B$, the smaller the gap between the exact and the approximated performance. Finally, the result in Figure 5.6 also confirms our analysis in Section 5.3, where the figure shows the sketch of asymptotic approximated SU's performance from ZFLP and the actual performance from ZFLP against $L=2^{B}$, comparing with the corresponding full CSI performance. Noticeably, the curve of approximated SU performance is improved but with slower rate for additional feedback bit and eventually reaches the performance of the full CSI case. The simulations show that SU performance from asymptotic approximation is indistinguishable from the simulated result from ZFLP algorithm for $B \geq 8$ and the performance is quite close to full-CSI case when $B \geq 10$.

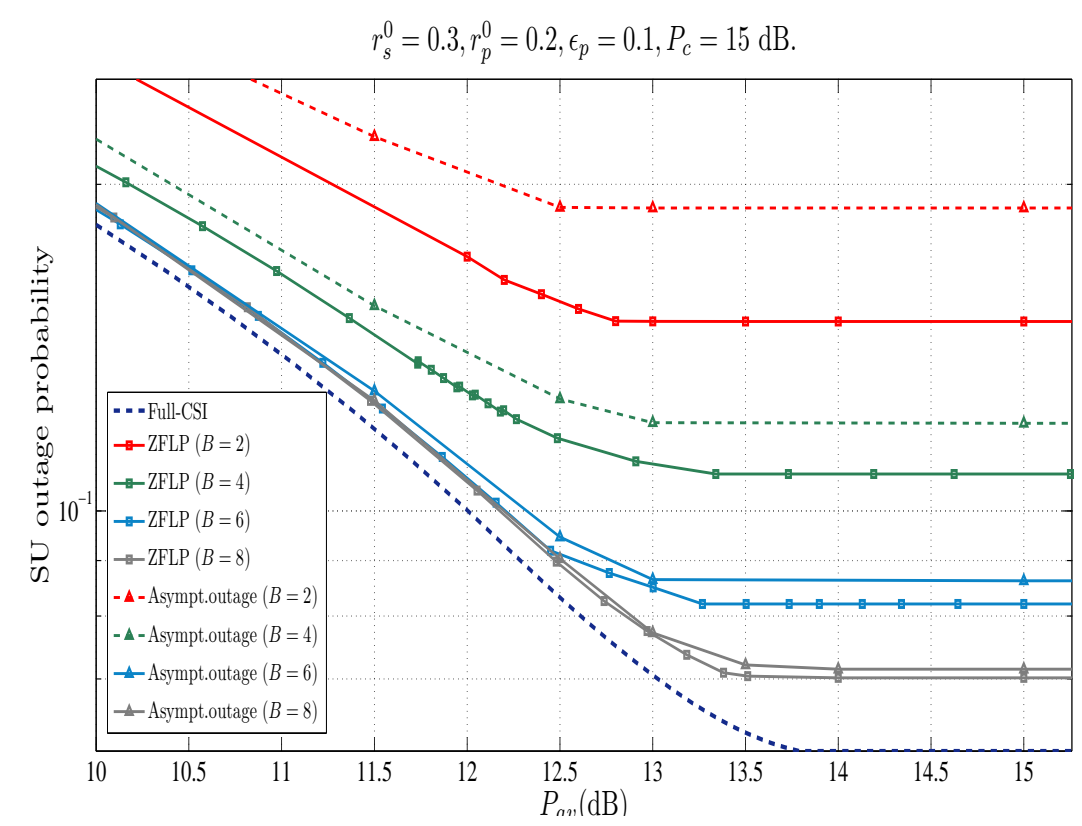

Figure 5.5: SU outage probability from ZFLP and from asymptotic analysis against average power $P_{a v}$ 


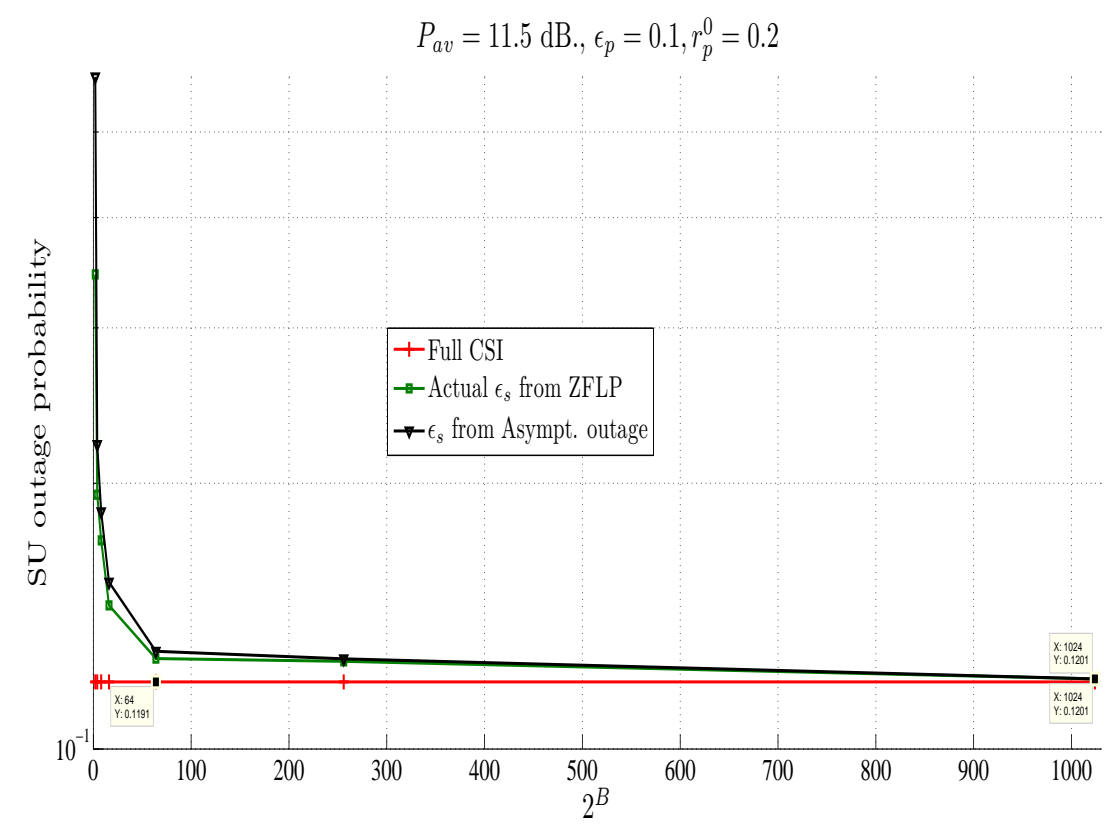

Figure 5.6: SU outage probability from ZFLP and from asymptotic analysis against $L=2^{B}$

\subsection{Conclusion}

This chapter has considered a cognitive radio network in underlay paradigm with a single pair of PU and SU, where the PU's service quality is protected by outage probability constraint. Assuming that the PU uses a constant power control, SU outage probability minimization problem subject to an ATPC and a POC under quantized feedback scenario has been investigated. First, the optimal channel structure for a given power codebook was obtained and then a locally optimal codebook was solved for by using the KKT necessary condition. Later, we proved that the lowest power level, $p_{L}$, approaches zero as $B$ grows large. This fact allowed us to propose another algorithm for suboptimal solution, called ZFLP, by assuming that $p_{L}=0$. The numerical results showed that the SU outage performance from ZFLP algorithm is close to the performance from a locally optimal solution for $B \geq 10$ but offers lower computational complexity. Also, ZFLP outperforms another suboptimal algorithm (GLA+SFA) for any given number of feedback bits. Finally, we approximated SU outage probability performance with the aid of several properties of a locally optimal power codebook when the number of bits grows large. The numerical results illustrated the difference between exact SU outage probability performance 
with our approximation against varying number of feedback bits, showing that the gap is nearly indistinguishable when $B \geq 8$. 


\section{Chapter 6}

\section{Conclusions}

This thesis investigated power-efficient resource alllocation strategies for underlay (or spectrum sharing) based cognitive radio networks in fading channels coexisting in the same frequency band with delay-sensitive primary user(s) where the service quality of each primary user is guaranteed by an outage probability constraint. Below, we will summarize our contributions and give possible ideas for future research related to the topics in this thesis.

\subsection{Summary}

In Chapter 2, we derived the optimal power allocation strategy to maximize the service-outage capacity for an OFDM-based secondary user in $N$ parallel fading channels where a distinct delay-sensitive primary user possesses a given subchannel. With perfect channel side information at the secondary transmitter, the solution was obtained by a probabilistic power allocation technique which reveals that the optimal power policy is randomized among $2^{N}+1$ deterministic power policies. The complexity of the optimal solution thus increases exponentially with the number of subchannels. Hence, we also proposed a suboptimal power strategy that is randomized among only 3 deterministic power policies. Performance comparisons between the sub-optmal and optimal solutions were presented.

In Chapter 3, we focused on a cognitive broadcast channel sharing the same spectrum band with a delay-sensitive primary user with perfect channel side information assumed at the secondary network. The optimal power policies to maximize the ergodic sum downlink capacity subject to either an average or a peak transmit power constraint were derived under continuous fading channel assumptions. The 
associated optimal strategy is that the secondary base station assigns the a given time slot (or equivalently a fading block over which the channel remains invariant) to only one user. Such a dynamic time-division multiple-access characteristic allows us to investigate further how the ergodic sum capacity scales as the number of secondary receivers, $M$, becomes large. We have shown that, if the PU adopts an ON-OFF power policy with constant power control when ON, the secondary ergodic sum throughput in BC scales according to $\log (\log M)$. If the PU uses a truncated channel inversion power control, the secondary sum throughput scales like $\epsilon_{p} \log (\log M)$ where $\epsilon_{p}$ is the PU outage probability threshold.

In Chapter 4, we studied continuous fading cognitive multiple-access channels with a real-time based primary user with full channel side information assumed at the secondary base station and the secondary transmitters. In Section 4.2, we focused on the ergodic capacity notion. We initially considered the special case of ergodic capacity acheiving region, i.e. SU ergodic sum rate maximization problem subject to POC and average transmit power constraint at each secondary transmitters. The optimal solution expresses that at most two users can simultaneously transmit. Later, we characterized the ergodic capacity achieving region with the similar types of constraints by using mathematical ingredients due to the polymatroid structure of the problem, showing that successive decoding is optimal. In Section 4.3 , we addressed two problems of outage capacity, i.e. common outage capacity and individual outage capacity. We proved that common outage capacity region and individual outage capacity region can be acquired by implicitly deriving the minimum common outage probability and the individual outage probability region, respectively, for a given rate vector. The optimal policies for both common outage probability and individual outage probability minimizing problems also reveal that successive decoding is optimal. Additionally, the effect of decoding order was illustrated via numerical simulations.

In Chapter 5, we examined the outage minimization problem for a single-antenna secondary user with a delay-sensitive primary user under quantized feedback. By using the nearest neighbourhood condition, the optimal channel partition structure can be derived for a given power codebook. Thus, the problem can be reformulated 
in terms of the power codebook and the locally optimal power codebook is solved accordingly by using necessary Karush-Kuhn-Tucker conditions. However, the complexity in computing a locally optimal power codebook increases as the number of feedback bits becomes large. In order to alleviate the burden on computational complexity, we also proposed a suboptimal algorithm called zero-forced lowest power level algorithm (ZFLP) by assuming that the lowest power level is zero, which is a property that is achieved by the power codebook as the number of feedback bits goes to infinity. In additon, an asymptotic analysis on the SU outage probability based on the ZFLP algorithm was given. Numerical results were provided to validate the performance of the ZFLP algorithm and the asymptotic approximation.

\subsection{Future research}

For the work on Chapter 2, the complexity to evaluate the optimal power in each channel state increases as the number of subchannels grows, hence a suboptimal algorithm was proposed. One can investigate power allocation schemes that can perform better than our proposed suboptimal algorithm. It is also possible to consider the optimal power control when each PU is not bounded to be just a delay-sensitive and may possess more than one subchannels. Further, there may be more than one PU operating in each subchannel. However, in order to analyze this case one requires the expression of the capacity of parallel interference channels which is still a largely unsolved problem. Also, when CSI at the OFDM-equipped secondary user is imperfect, one can extend this work by analyzing the power control under quantized or noisy channel feedback.

In the work on Chapter 3 and Chapter 4, we have considered the multiuser scenario in secondary network with only a single primary user, so it is possible to extend this work to the case of multiple primary users in the primary network. Also, CSI of the entire network is presumed to be available at each transmitter which is hard to obtain in practice. Therefore, the more challenging problem is how to design efficient power control policies given that only quantized channel feedback is available. Essentially in typical broadcast channels where the receivers do not 
cooperate and the number of bits fed back to the base station increases with the number of receivers. It is worth mentioning that one can consider the throughput scaling analyses for cognitive MAC in the similar manner as we did for cognitive $\mathrm{BC}$ in Chapter 3. Other types of wireless channel models, such as Nakagami-m or Rician fading, can be considered in the future extensions as the channel model obviously affects the throughput scaling result. Another intriguing problem is to elaborate the resource allocation problem to a cognitive MIMO network with a delay-sensitive primary network since in this case both a beamforming codebook and power codebook must be appropriately designed.

Finally, the work on Chapter 5 can be extended to the case when there is no band manager and the secondary transmitter acquires partial side information via receiving quantized feedback directly from the secondary receiver and the primary receiver separately and the quantized feedback design from both terminals is required for the secondary network. In brief, one can further analyze the problem using separate scalar quantization, as opposed to vector quantization that we have considered in Chapter 5. Besides, other utility functions can replace the outage probability. For example, secondary ergodic capacity maximization subject to primary user's outage constraint and average transmit power constraint under limited feedback is still unexplored. Another possibility for extending this work is to analyze more generalized scenarios, such as when the secondary user is equipped with OFDM technology like the work in Chapter 2 but within a limited feedback scenario, or when each secondary terminal is equipped with multiple antennas, or even when there are multiple secondary and/or primary users, e.g. cognitive BC or cognitive MAC under a quatized feedback setup. 


\section{Appendix A}

\section{Proofs in Chapter 2}

\section{A.1 Proof of Lemma 2.2.1}

We first show that for an arbitrary feasible probabilistic power scheme $\mathbf{P}_{s}(\boldsymbol{\nu})$, we can always construct another feasible scheme $\mathbf{P}_{s}^{\prime}(\boldsymbol{\nu})$ which is randomized among deterministic power schemes $p_{k i}(\boldsymbol{\nu})$ with time-sharing factors $w_{k i}(\boldsymbol{\nu})$, and performs equally well or better than $\mathbf{P}_{s}(\boldsymbol{\nu})$. Since $\mathbf{P}_{s}(\boldsymbol{\nu})$ is feasible, $\mathbf{P}_{s}(\boldsymbol{\nu})$ satisfies all the constraints, i.e. $E\left[\left\langle\boldsymbol{P}_{s i}(\boldsymbol{\nu})\right\rangle\right] \leq P_{a v}, \operatorname{Pr}\left\{r_{p i}\left(\boldsymbol{\nu}, P_{s i}(\boldsymbol{\nu})\right)<r_{p i}^{0}\right\} \leq \epsilon_{p i}$, and $\operatorname{Pr}\left\{\sum_{i=1}^{N} r_{s i}\left(\boldsymbol{\nu}, P_{s i}(\boldsymbol{\nu})\right)<r_{s}^{0}\right\} \leq$ $\epsilon_{s}$. By the definitions of $p_{k i}(\boldsymbol{\nu})$ in $(2.4)$ and $w_{k i}(\boldsymbol{\nu})$ in (2.3), we have the following condition:

$$
\begin{aligned}
r_{s}^{0} & \leq E\left[\sum_{i=1}^{N} r_{s i}\left(\boldsymbol{\nu}, P_{s i}(\boldsymbol{\nu})\right) \mid \sum_{i=1}^{N} r_{s i}\left(\boldsymbol{\nu}, p_{s i}(\boldsymbol{\nu})\right) \geq r_{s}^{0}, \boldsymbol{\nu}\right] \\
& \stackrel{(a)}{\leq} \sum_{i=1}^{N} r_{s i}\left(\boldsymbol{\nu}, E\left[P_{s i}(\boldsymbol{\nu}) \mid \sum_{i=1}^{N} r_{s i}\left(\boldsymbol{\nu}, p_{s i}(\boldsymbol{\nu})\right) \geq r_{s}^{0}, \boldsymbol{\nu}\right]\right) \\
& \stackrel{(b)}{=} \sum_{i=1}^{N} r_{s i}\left(\boldsymbol{\nu}, \frac{w_{1 i}(\boldsymbol{\nu})}{w_{a}(\boldsymbol{\nu})} p_{1 i}(\boldsymbol{\nu})+\frac{w_{a}(\boldsymbol{\nu})-w_{1 i}(\boldsymbol{\nu})}{w_{a}(\boldsymbol{\nu})} p_{2 i}(\boldsymbol{\nu})\right)
\end{aligned}
$$

where $(a)$ follows from Jensen's inequality since $\sum_{i=1}^{N} r_{s i}\left(\boldsymbol{\nu}, p_{s i}(\boldsymbol{\nu})\right)$ is concave over $\boldsymbol{p}_{s}(\boldsymbol{\nu})$ and $(b)$ follows from the fact that $E\left[P_{s i}(\boldsymbol{\nu}) \mid \sum_{i=1}^{N} r_{s i}\left(\boldsymbol{\nu}, p_{s i}(\boldsymbol{\nu})\right) \geq r_{s}^{0}, \boldsymbol{\nu}\right]=\frac{w_{1 i}(\boldsymbol{\nu})}{w_{a}(\boldsymbol{\nu})} p_{1 i}(\boldsymbol{\nu})+$ $\frac{w_{a}(\boldsymbol{\nu})-w_{1 i}(\boldsymbol{\nu})}{w_{a}(\boldsymbol{\nu})} p_{2 i}(\boldsymbol{\nu})$ by conditional expectation.

Furthermore, for all $i, \operatorname{Pr}\left\{P_{s i}^{\prime}(\boldsymbol{\nu})=p_{1 i}(\boldsymbol{\nu})\right\}=w_{1 i}(\boldsymbol{\nu})$ and $\operatorname{Pr}\left\{P_{s i}^{\prime}(\boldsymbol{\nu})=p_{2 i}(\boldsymbol{\nu})\right\}=$ $w_{2 i}(\boldsymbol{\nu})$. Hence, $w_{1 i}(\boldsymbol{\nu})+w_{2 i}(\boldsymbol{\nu})=\operatorname{Pr}\left\{P_{s i}^{\prime}(\boldsymbol{\nu})=E\left[P_{s i}(\boldsymbol{\nu}) \mid \sum_{i=1}^{N} r_{s i}\left(\boldsymbol{\nu}, p_{s i}(\boldsymbol{\nu})\right) \geq r_{s}^{0}, \boldsymbol{\nu}\right] \mid \boldsymbol{\nu}\right\}=$ $w_{a}(\boldsymbol{\nu})$ for all $i$.

In other words, $\operatorname{Pr}\left\{\mathbf{P}_{s}^{\prime}(\boldsymbol{\nu})=E\left[\mathbf{P}_{s}(\boldsymbol{\nu}) \mid \sum_{i=1}^{N} r_{s i}\left(\boldsymbol{\nu}, p_{s i}(\boldsymbol{\nu})\right) \geq r_{s}^{0}, \boldsymbol{\nu}\right] \mid \boldsymbol{\nu}\right\}=w_{a}(\boldsymbol{\nu})$. Therefore,

$\operatorname{Pr}\left\{\sum_{i=1}^{N} r_{s i}\left(\boldsymbol{\nu}, P_{s i}^{\prime}(\boldsymbol{\nu})\right) \geq r_{s}^{0} \mid \boldsymbol{\nu}\right\} \geq w_{a}(\boldsymbol{\nu})$ and $E\left[\operatorname{Pr}\left\{\sum_{i=1}^{N} r_{s i}\left(\boldsymbol{\nu}, P_{s i}^{\prime}(\boldsymbol{\nu})\right) \geq r_{s}^{0} \mid \boldsymbol{\nu}\right\}\right] \geq$ $E\left[w_{a}(\boldsymbol{\nu})\right] \geq 1-\epsilon_{s}$. This implies that the new power scheme $\mathbf{P}_{s}^{\prime}(\boldsymbol{\nu})$, if feasible, results 
in an SU outage probability performance that will not be worse than that of $\mathbf{P}_{s}(\boldsymbol{\nu})$.

Now, we will show that $r_{p i}\left(\boldsymbol{\nu}, E\left[P_{s i}(\boldsymbol{\nu}) \mid r_{p i}\left(\boldsymbol{\nu}, p_{s i}(\boldsymbol{\nu})\right) \geq r_{p i}^{0}, \boldsymbol{\nu}\right]\right) \geq r_{p i}^{0}$. Note that when $r_{p i}\left(\boldsymbol{\nu}, p_{s i}(\boldsymbol{\nu})\right) \geq r_{p i}^{0}$, it implies $p_{s i}(\boldsymbol{\nu}) \leq \frac{1}{\beta_{i}}\left(\frac{g_{i} P_{p i}\left(g_{i}\right)}{e^{r p i}-1}-N_{0}\right)^{+}$, i.e. the possible solution lies in a halfspace. Further, notice that $E\left[P_{s i}(\boldsymbol{\nu}) \mid r_{p i}\left(\boldsymbol{\nu}, p_{s i}(\boldsymbol{\nu})\right) \geq r_{p i}^{0}, \boldsymbol{\nu}\right]$ is a convex combination of the possible solutions in that halfspace weighted by the probability that each solution can happen. So, $E\left[P_{s i}(\boldsymbol{\nu}) \mid r_{p i}\left(\boldsymbol{\nu}, p_{s i}(\boldsymbol{\nu})\right) \geq r_{p i}^{0}, \boldsymbol{\nu}\right]$ also lies in the same halfspace due to convex set properties, which means

$$
\begin{aligned}
r_{p i}^{0} & \leq r_{p i}\left(\boldsymbol{\nu}, E\left[P_{s i}(\boldsymbol{\nu}) \mid r_{p i}\left(\boldsymbol{\nu}, p_{s i}(\boldsymbol{\nu})\right) \geq r_{p i}^{0}, \boldsymbol{\nu}\right]\right) \\
& \stackrel{(c)}{=} r_{p i}\left(\boldsymbol{\nu}, \frac{w_{1 i}(\boldsymbol{\nu})}{w_{1 i}(\boldsymbol{\nu})+w_{3 i}(\boldsymbol{\nu})} p_{1 i}(\boldsymbol{\nu})+\frac{w_{1 i}(\boldsymbol{\nu})}{w_{3 i}(\boldsymbol{\nu})+w_{3 i}(\boldsymbol{\nu})} p_{3 i}(\boldsymbol{\nu})\right)
\end{aligned}
$$

where $(c)$ follows again from the definition of conditional expectation.

In addition, $\operatorname{Pr}\left\{P_{s i}^{\prime}(\boldsymbol{\nu})=E\left[P_{s i}(\boldsymbol{\nu}) \mid r_{p i}\left(\boldsymbol{\nu}, p_{s i}(\boldsymbol{\nu})\right) \geq r_{p i}^{0}, \boldsymbol{\nu}\right] \mid \boldsymbol{\nu}\right\}=w_{1 i}(\boldsymbol{\nu})+w_{3 i}(\boldsymbol{\nu})$ and it can be shown that $E\left[\operatorname{Pr}\left\{r_{p i}\left(\boldsymbol{\nu}, P_{s i}^{\prime}(\boldsymbol{\nu})\right) \geq r_{p i}^{0} \mid \boldsymbol{\nu}\right\}\right] \geq E\left[w_{1 i}(\boldsymbol{\nu})+w_{3 i}(\boldsymbol{\nu})\right] \geq$ $1-\epsilon_{p i}$.

Also, $E\left[\left\langle\boldsymbol{P}_{s}^{\prime}(\boldsymbol{\nu})\right\rangle\right]=E\left[\sum_{i=1}^{N} \sum_{k=1}^{4} w_{k i}(\boldsymbol{\nu}) p_{k i}(\boldsymbol{\nu})\right]=E\left[\left\langle\boldsymbol{P}_{s}(\boldsymbol{\nu})\right\rangle\right]$. The feasibility of $\mathbf{P}_{s}^{\prime}(\boldsymbol{\nu})$ has thus been proved. Finally, it can be shown that $E\left[\sum_{i=1}^{N} r_{s i}\left(\boldsymbol{\nu}, P_{s i}^{\prime}(\boldsymbol{\nu})\right)\right]=$ $E\left[\sum_{i=1}^{N} \sum_{k=1}^{4} w_{k i}(\boldsymbol{\nu}) r_{s i}\left(\boldsymbol{\nu}, p_{k i}(\boldsymbol{\nu})\right)\right] \geq E\left[\sum_{i=1}^{N} r_{s i}\left(\boldsymbol{\nu}, P_{s i}(\boldsymbol{\nu})\right)\right]$, where the inequality follows from Jensen's inequality. This completes the proof.

\section{A.2 Proof of convexity of (2.5)}

Here we suppress the dependence of the determistic power policies and the weighting functions on $\boldsymbol{\nu}$. Let $x_{k i}=w_{k i} p_{k i}$ where $w_{2 i}=w_{a}-w_{1 i}$. Then we can map $\left(p_{1 i}, p_{2 i}, p_{3 i}, p_{4 i}, w_{1 i}, w_{3 i}, w_{4 i}, w_{a}\right)$ to $\left(x_{1}, x_{2}, x_{3}, x_{4}, w_{1 i}, w_{3 i}, w_{4 i}, w_{a}\right)$, such that $(2.5)$ 
can be rewritten as

$$
\begin{array}{cl}
\max _{\mathscr{Z}\left(x_{k i}, w_{k i}, \forall k, \forall i\right)} & E\left[\sum_{i=1}^{N} \sum_{k=1}^{4} w_{k i} r_{s}\left(\nu, \frac{x_{k i}}{w_{k i}}\right)\right] \\
\text { s.t. } & \text { (a) } E\left[\sum_{i=1}^{N} \sum_{k=1}^{4} x_{k i}\right] \leq P_{a v}, \quad \text { (b) } E\left[w_{1 i}+w_{3 i}\right] \geq 1-\epsilon_{p i}, \\
& \text { (c) } E\left[w_{1 i}+w_{2 i}\right]=E\left[w_{a}\right] \geq 1-\epsilon_{s}, \\
& \text { (d) } \sum_{i=1}^{N} r_{s i}\left(\boldsymbol{\nu}, \frac{w_{1 i}}{w_{a}} \frac{x_{1 i}}{w_{1 i}}+\frac{\left(w_{a}-w_{1 i}\right)}{w_{a}} \frac{x_{2 i}}{w_{a}-w_{1 i}}\right)-r_{s}^{0} \geq 0, \\
& \text { (e) } w_{1 i} p_{r p, i}(\nu)+w_{3 i} p_{r p, i}(\nu)-x_{1 i}-x_{3 i} \geq 0, \quad \text { (f) } w_{a}-w_{1 i}=w_{2 i} \geq 0, \\
& \text { (g) } 1-w_{a}-w_{3 i}=w_{4 i} \geq 0, \quad \text { (h) } x_{k i} \geq 0, \quad \text { (i) } w_{k i} \geq 0 .
\end{array}
$$

Note that in the objective function of $(\mathrm{A} .1), w_{k i} r_{s}\left(\nu, \frac{x_{k i}}{w_{k i}}\right)$ is concave over $\left(x_{k i}, w_{k i}\right)$ (see [25]). To show that (A.1d) is convex, we can rearrange the inequality to be $\prod_{i=1}^{N}\left[w_{a}+\frac{h_{i}}{\alpha P_{p i}\left(g_{i}\right)+N_{0}}\left(x_{1 i}+x_{2 i}\right)\right]^{1 / N} \geq w_{a} e^{\frac{r_{s}^{0}}{N}}$. Since $t(z)=\left(\prod_{i=1}^{K} z\right)^{1 / N}$ is concave on $z \in \Re_{+}^{K}$ [90], the left side of the inequality is concave with respect to $\left(w_{a}, x_{1 i}, x_{2 i}\right)$, while the right hand side is linear over $w_{a}$. This results in a convex constraint when written in the standard form. The convexity of the rest of the constraints are obvious. This proves the convexity of $(2.5)$.

\section{A.3 Proof of Theorem 2.3.1}

We will first solve for $p_{k i}^{*}(\boldsymbol{\nu})$ to show that there are $2^{N}+1$ possible deterministic optimal power schemes $\mathbf{p}^{*}(\boldsymbol{\nu})$ and then derive the solution for all optimal weighting functions. With $w_{k i}^{*}(\boldsymbol{\nu})=0$, the solution of $p_{k i}^{*}(\boldsymbol{\nu})$ has no impact on the optimization problem. Hence, we will consider the case $w_{k i}^{*}(\boldsymbol{\nu})>0$ in order to solve for $p_{k i}^{*}(\boldsymbol{\nu})$. To avoid the repetition, we will show the derivation for $p_{1 i}^{*}(\boldsymbol{\nu})$ only and the finalized expressions for all $p_{k i}^{*}(\boldsymbol{\nu})$ for $k=2,3,4$.

$$
\operatorname{By} \frac{\partial \sum_{i=1}^{N} r_{s i}\left(\boldsymbol{\nu}, \frac{w_{1 i}^{*}(\boldsymbol{\nu})}{w_{a}^{*}(\boldsymbol{\nu})} p_{1 i}^{*}(\boldsymbol{\nu})+\frac{w_{a}^{*}(\boldsymbol{\nu})-w_{1 i}^{*}(\boldsymbol{\nu})}{w_{a}^{*}(\boldsymbol{\nu})} p_{2 i}^{*}(\boldsymbol{\nu})\right)}{\partial p_{1 i}^{*}(\boldsymbol{\nu})}=\frac{w_{1 i}^{*}(\boldsymbol{\nu})}{w_{a}^{*}(\boldsymbol{\nu})} \frac{1}{\frac{w_{1 i}^{*}(\boldsymbol{\nu})}{w_{a}^{a}(\boldsymbol{\nu})} p_{1 i}^{*}(\boldsymbol{\nu})+\frac{w_{a}^{*}(\boldsymbol{\nu})-w_{1 i}^{*}(\boldsymbol{\nu})}{w_{a}^{*}(\boldsymbol{\nu})} p_{2 i}^{*}(\boldsymbol{\nu})+\frac{\alpha_{i} P_{p i}\left(g_{i}\right)+N_{0}}{h_{i}}},
$$

we apply the condition in $(2.7)$, we can now solve for $p_{1 i}^{*}(\boldsymbol{\nu})\left(\right.$ when $w_{1 i}^{*}(\boldsymbol{\nu})>0$ ) as follows:

- When $q^{*}(\boldsymbol{\nu})=0$ and $u_{i}^{*}(\boldsymbol{\nu})=0$

It yields $\frac{1}{p_{1 i}^{*}(\boldsymbol{\nu})+\frac{\alpha_{i} P_{p i}\left(g_{i}\right)+N_{0}}{h_{i}}}-\lambda^{*}=0$ and thus $p_{1 i}^{*}(\boldsymbol{\nu})=p_{w f, i}\left(\boldsymbol{\nu}, \lambda^{*}\right)$. 
- When $q^{*}(\boldsymbol{\nu})=0$ and $u_{i}^{*}(\boldsymbol{\nu})>0$

In this case $\left[\frac{1}{p_{1 i}^{*}(\boldsymbol{\nu})+\frac{\alpha P_{p i}\left(g_{i}\right)+N_{0}}{h_{i}}}-\lambda^{*}\right]-u_{i}^{*}(\boldsymbol{\nu})=0$, and $w_{1 i}^{*}(\boldsymbol{\nu})\left(p_{r p, i}-p_{1 i}^{*}(\boldsymbol{\nu})\right)+$ $w_{3 i}^{*}(\boldsymbol{\nu})\left(p_{r p, i}-p_{3 i}^{*}(\boldsymbol{\nu})\right)=0$ by $u_{i}^{*}(\boldsymbol{\nu})>0$ from the associated KKT conditions. Suppose both $w_{1 i}^{*}(\boldsymbol{\nu})$ and $w_{3 i}^{*}(\boldsymbol{\nu})$ are positive. Let $p_{1 i}^{*}(\boldsymbol{\nu})<p_{r p, i}$, which leads to $p_{3 i}^{*}(\boldsymbol{\nu})>p_{r p, i}$ which contradicts the definition in (2.4). Similarly, we cannot let $p_{1 i}^{*}(\boldsymbol{\nu})>p_{r p, i}$ using the same argument. Then one must have $p_{1 i}^{*}(\boldsymbol{\nu})=p_{3 i}^{*}(\boldsymbol{\nu})=$ $p_{r p, i}(\boldsymbol{\nu})$. Again, by (2.4), we can conclude that, when $q^{*}(\boldsymbol{\nu})=0$ and $u_{i}^{*}(\boldsymbol{\nu})>0$, both $w_{1 i}^{*}(\boldsymbol{\nu})$ and $w_{3 i}^{*}(\boldsymbol{\nu})$ cannot be positive, since identical values of $p_{1 i}^{*}(\boldsymbol{\nu})$ and $p_{3 i}^{*}(\boldsymbol{\nu})$ cannot achieve the service rate for the $\mathrm{SU}$ on one hand (definition of $\left.p_{1 i}^{*}(\boldsymbol{\nu})\right)$ and result in an outage for the $\mathrm{SU}$ on the other hand (definition of $\left.p_{3 i}^{*}(\boldsymbol{\nu})\right)$.

\section{- When $q^{*}(\boldsymbol{\nu})>0$}

First, let $w_{1 i}^{*}(\boldsymbol{\nu})>0$ and $w_{a}^{*}(\boldsymbol{\nu})-w_{1 i}^{*}(\boldsymbol{\nu})>0$. Then one should have $\frac{w_{1 i}^{*}(\boldsymbol{\nu})}{w_{a}^{*}(\boldsymbol{\nu})} p_{1 i}^{*}(\boldsymbol{\nu})+$ $\frac{w_{a}^{*}(\boldsymbol{\nu})-w_{1 i}^{*}(\boldsymbol{\nu})}{w_{a}^{*}(\boldsymbol{\nu})} p_{2 i}^{*}(\boldsymbol{\nu})=p_{r s, i}(\boldsymbol{\nu})$ in every $i$-th subchannel in order to achieve minimum power consumption.

Thus, we have

$$
\begin{array}{lll} 
& \frac{w_{1 i}^{*}(\boldsymbol{\nu})}{w_{a}^{*}(\boldsymbol{\nu})} r_{s i}\left(\boldsymbol{\nu}, p_{1 i}^{*}(\boldsymbol{\nu})\right)+\frac{w_{a}^{*}(\boldsymbol{\nu})-w_{1 i}^{*}(\boldsymbol{\nu})}{w_{a}^{*}(\boldsymbol{\nu})} r_{s i}\left(\boldsymbol{\nu}, p_{2 i}^{*}(\boldsymbol{\nu})\right) & \stackrel{(d)}{\leq} r_{s i}\left(\boldsymbol{\nu}, p_{r s, i}(\boldsymbol{\nu})\right) \\
\therefore \sum_{i=1}^{N}\left[\frac{w_{1 i}^{*}(\boldsymbol{\nu})}{w_{a}^{*}(\boldsymbol{\nu})} r_{s i}\left(\boldsymbol{\nu}, p_{1 i}^{*}(\boldsymbol{\nu})\right)+\frac{w_{a}^{*}(\boldsymbol{\nu})-w_{1 i}^{*}(\boldsymbol{\nu})}{w_{a}^{*}(\boldsymbol{\nu})} r_{s i}\left(\boldsymbol{\nu}, p_{2 i}^{*}(\boldsymbol{\nu})\right)\right] & \leq r_{s}^{0}
\end{array}
$$

where $(d)$ follows from Jensen's inequality.

The above result implies that if SU randomly switches between the strategies $p_{1 i}^{*}(\boldsymbol{\nu})$ and $p_{2 i}^{*}(\boldsymbol{\nu})$, the average SU rate in that specific $\boldsymbol{\nu}$ will be less than $r_{s}^{0}$. But from the definition, we know that $w_{a}^{*}(\boldsymbol{\nu})=\operatorname{Pr}\left(\sum_{i=1}^{N} r_{s i}\left(\boldsymbol{\nu}, p_{r s, i}(\boldsymbol{\nu})\right) \geq r_{s}^{0}\right)$. Hence the only possibility is that the equality is met if and only if $p_{1 i}^{*}(\boldsymbol{\nu})=$ $p_{2 i}^{*}(\boldsymbol{\nu})=p_{r s, i}(\boldsymbol{\nu})$. However, this is impossible due to the definition in (2.4). Hence, to achieve $r_{s}^{0}$ with probability $w_{a}^{*}(\boldsymbol{\nu})$ for a given $\boldsymbol{\nu}$, it can be done by

1. either $w_{1 i}^{*}(\boldsymbol{\nu})=w_{a}^{*}(\boldsymbol{\nu}), w_{2 i}^{*}(\boldsymbol{\nu})=0, p_{1 i}^{*}(\boldsymbol{\nu})=p_{r s, i}(\boldsymbol{\nu})$,

2. or $w_{1 i}^{*}(\boldsymbol{\nu})=0, w_{2 i}^{*}(\boldsymbol{\nu})=w_{a}^{*}(\boldsymbol{\nu}), p_{2 i}^{*}(\boldsymbol{\nu})=p_{r s, i}(\boldsymbol{\nu})$. 
Now, we will consider the two sub-cases when $u_{i}^{*}(\boldsymbol{\nu})>0$ and $u_{i}^{*}(\boldsymbol{\nu})=0$

- When $q^{*}(\boldsymbol{\nu})>0$ and $u_{i}^{*}(\boldsymbol{\nu})>0$

As $u_{i}^{*}(\boldsymbol{\nu})>0 p_{1 i}^{*}(\boldsymbol{\nu})=p_{r p, i}$. However, this optimal power $p_{1 i}^{*}(\boldsymbol{\nu})=p_{r p, i}$ is not one of the conditions for $q^{*}(\boldsymbol{\nu})>0$ discussed earlier, unless $p_{r p, i}=p_{r s, i}$ with positive probability, which is not possible for a continuous fading scenario. Although it is possible that $p_{r p, i}=p_{r s, i}$ for a discrete fading scenario, this will not affect the optimal solution.

- When $q^{*}(\boldsymbol{\nu})>0$ and $u_{i}^{*}(\boldsymbol{\nu})=0$

Then, From the three conditions previously discussed, it shows that if both $w_{1 i}^{*}(\boldsymbol{\nu}), w_{2 i}^{*}(\boldsymbol{\nu})>0$, then $p_{1 i}^{*}(\boldsymbol{\nu})=p_{2 i}^{*}(\boldsymbol{\nu})=p_{r s, i}(\boldsymbol{\nu})$, (by considering the the optimality conditions when $q^{*}(\boldsymbol{\nu})>0$ and $p_{2 i}^{*}(\boldsymbol{\nu})>0$ ), which is impossible by definition. So, if $q^{*}(\boldsymbol{\nu})>0$ and $w_{1 i}^{*}(\boldsymbol{\nu})>0$, then $p_{1 i}^{*}(\boldsymbol{\nu})=p_{r s, i}(\boldsymbol{\nu})$ while $w_{2 i}^{*}(\boldsymbol{\nu})=0$. Furthermore, it is impossible for $p_{1 i}^{*}(\boldsymbol{\nu})=p_{r s, i}(\boldsymbol{\nu})$ to hold when $\mathbf{p}_{r s}(\boldsymbol{\nu}) \prec \mathbf{p}_{w f}\left(\boldsymbol{\nu}, \lambda^{*}\right)$ because $p_{r s, i}^{*}(\boldsymbol{\nu})=\left(\frac{1+\frac{q^{*}(\boldsymbol{\nu})}{\left.w_{*}^{*} \boldsymbol{\nu}\right)}}{\lambda^{*}}-\frac{\alpha_{i} P_{p i}\left(g_{i}\right)+N_{0}}{h_{i}}\right)^{+}>p_{w f, i}^{*}\left(\boldsymbol{\nu}, \lambda^{*}\right), \forall i$. Note that here we have $\mu(\boldsymbol{\nu})=\frac{1+\frac{q^{*}(\boldsymbol{\nu})}{w_{a}^{*}(\boldsymbol{\nu})}}{\lambda^{*}}$.

Applying a similar procedure to $p_{k i}^{*}(\boldsymbol{\nu})$, for $k=2,3,4$, we obtain the closed form solutions of all $p_{k i}^{*}(\boldsymbol{\nu})$ as follows

$$
\begin{gathered}
p_{1 i}^{*}(\boldsymbol{\nu})= \begin{cases}p_{w f, i}\left(\boldsymbol{\nu}, \lambda^{*}\right), & w_{1 i}^{*}(\boldsymbol{\nu})>0, \mathbf{p}_{r s}(\boldsymbol{\nu}) \preceq \mathbf{p}_{w f}\left(\boldsymbol{\nu}, \lambda^{*}\right), p_{w f, i}\left(\boldsymbol{\nu}, \lambda^{*}\right) \leq p_{r p, i}(\boldsymbol{\nu}) \\
p_{r s, i}(\boldsymbol{\nu}), & w_{1 i}^{*}(\boldsymbol{\nu})>0, \mathbf{p}_{w f}\left(\boldsymbol{\nu}, \lambda^{*}\right) \prec \mathbf{p}_{r s}(\boldsymbol{\nu}), p_{w f, i}\left(\boldsymbol{\nu}, \lambda^{*}\right) \leq p_{r p, i}(\boldsymbol{\nu}) \\
p_{r p, i}(\boldsymbol{\nu}), & w_{1 i}^{*}(\boldsymbol{\nu})>0, \mathbf{p}_{r s}(\boldsymbol{\nu}) \preceq \mathbf{p}_{w f}\left(\boldsymbol{\nu}, \lambda^{*}\right), p_{w f, i}\left(\boldsymbol{\nu}, \lambda^{*}\right)>p_{r p, i}(\boldsymbol{\nu})\end{cases} \\
p_{2 i}^{*}(\boldsymbol{\nu})= \begin{cases}p_{w f, i}\left(\boldsymbol{\nu}, \lambda^{*}\right), & w_{a}^{*}(\boldsymbol{\nu})-w_{1 i}^{*}(\boldsymbol{\nu})>0, \mathbf{p}_{r s}(\boldsymbol{\nu}) \preceq \mathbf{p}_{w f}\left(\boldsymbol{\nu}, \lambda^{*}\right) \\
p_{r s, i}(\boldsymbol{\nu}), & w_{a}^{*}(\boldsymbol{\nu})-w_{1 i}^{*}(\boldsymbol{\nu})>0, \mathbf{p}_{w f}\left(\boldsymbol{\nu}, \lambda^{*}\right) \prec \mathbf{p}_{r s}(\boldsymbol{\nu})\end{cases} \\
p_{3 i}^{*}(\boldsymbol{\nu})= \begin{cases}p_{w f, i}\left(\boldsymbol{\nu}, \lambda^{*}\right), & w_{3 i}^{*}(\boldsymbol{\nu})>0, p_{w f, i}\left(\boldsymbol{\nu}, \lambda^{*}\right) \leq p_{r p, i}(\boldsymbol{\nu}) \\
p_{r p, i}(\boldsymbol{\nu}), & w_{3 i}^{*}(\boldsymbol{\nu})>0, p_{w f, i}\left(\boldsymbol{\nu}, \lambda^{*}\right)>p_{r p, i}(\boldsymbol{\nu}) \\
p_{4 i}^{*}(\boldsymbol{\nu})=p_{w f, i}\left(\boldsymbol{\nu}, \lambda^{*}\right), & w_{4 i}^{*}(\boldsymbol{\nu})>0\end{cases}
\end{gathered}
$$


Now, we will derive optimal solutions for $w_{1 i}^{*}(\boldsymbol{\nu}), w_{3 i}^{*}(\boldsymbol{\nu})$, and $w_{a}^{*}(\boldsymbol{\nu})$.

$w_{3 i}^{*}(\boldsymbol{\nu})$

Regardless of whether $q^{*}(\boldsymbol{\nu})$ is zero or strictly positive, we have $\frac{\partial l(\ldots)}{\left.\partial w_{3 i}^{*}(\boldsymbol{\nu})\right)}=s_{i}+$ $r_{s i}\left(\boldsymbol{\nu}, p_{3 i}(\boldsymbol{\nu})\right)-\lambda p_{3 i}(\boldsymbol{\nu})-\left(r_{s i}\left(\boldsymbol{\nu}, p_{4 i}(\boldsymbol{\nu})\right)-\lambda p_{4 i}(\boldsymbol{\nu})\right)-\eta_{i}^{*}(\boldsymbol{\nu})$ (note that when $u_{i}^{*}(\boldsymbol{\nu})>0$, the term $u_{i}^{*}(\boldsymbol{\nu})\left(p_{r p, i}(\boldsymbol{\nu})-p_{3 i}^{*}\right)=0$ from optimality conditions). Hence from the KKT conditions, it follows that

$$
w_{3 i}^{*}(\boldsymbol{\nu})\left\{\begin{array}{cc}
=0, & s_{i}+r_{s i}\left(\boldsymbol{\nu}, p_{3 i}(\boldsymbol{\nu})\right)-\lambda p_{3 i}(\boldsymbol{\nu}) \leq\left(r_{s i}\left(\boldsymbol{\nu}, p_{4 i}(\boldsymbol{\nu})\right)-\lambda p_{4 i}(\boldsymbol{\nu})\right)+\eta_{i}^{*}(\boldsymbol{\nu}) \\
>0, & s_{i}+r_{s i}\left(\boldsymbol{\nu}, p_{3 i}(\boldsymbol{\nu})\right)-\lambda p_{3 i}(\boldsymbol{\nu})=\left(r_{s i}\left(\boldsymbol{\nu}, p_{4 i}(\boldsymbol{\nu})\right)-\lambda p_{4 i}(\boldsymbol{\nu})\right)+\eta_{i}^{*}(\boldsymbol{\nu})
\end{array}\right.
$$

\section{Note for $w_{3 i}^{*}(\boldsymbol{\nu})$ and $w_{4 i}^{*}(\boldsymbol{\nu})$}

- Suppose that $1-w_{a}^{*}(\boldsymbol{\nu})>0$. From (A.7),

$$
\begin{aligned}
\frac{\partial l(\ldots)}{\partial w_{3 i}^{*}(\boldsymbol{\nu})}=0 & \Rightarrow w_{3 i}^{*}>0, \mathscr{B}_{3 i, \boldsymbol{\nu}}=\mathscr{B}_{4 i, \boldsymbol{\nu}}+\eta_{i}^{*}(\boldsymbol{\nu}) \geq \mathscr{B}_{4 i, \boldsymbol{\nu}} \\
& \Rightarrow\left\{\begin{array}{l}
w_{4 i}=0, \quad \mathscr{B}_{3 i, \boldsymbol{\nu}} \geq \mathscr{B}_{4 i, \boldsymbol{\nu}}, \eta_{i}^{*} \geq 0 \\
w_{4 i}>0, \quad \mathscr{B}_{3 i, \boldsymbol{\nu}}=\mathscr{B}_{4 i, \boldsymbol{\nu}}, \eta_{i}^{*}=0
\end{array}\right. \\
\frac{\partial l(\ldots)}{\partial w_{3 i}^{*}(\boldsymbol{\nu})} \leq 0 & \Rightarrow w_{3 i}^{*}=0, \mathscr{B}_{3 i, \boldsymbol{\nu}} \leq \mathscr{B}_{4 i, \boldsymbol{\nu}}+\eta_{i}^{*}=\mathscr{B}_{4 i, \boldsymbol{\nu}}, \quad \text { as } \eta_{i}^{*}=0
\end{aligned}
$$

Then, for every $\mathscr{B}_{4 i, \boldsymbol{\nu}}+\eta_{i}^{*}$, it can be replaced by either $\mathscr{B}_{3 i, \boldsymbol{\nu}}$ or $\mathscr{B}_{4 i, \boldsymbol{\nu}}$.

- If $1-w_{a}^{*}(\boldsymbol{\nu})=0, w_{3 i}^{*}(\boldsymbol{\nu})=w_{4 i}^{*}(\boldsymbol{\nu})=0$

Also, $\mathscr{B}_{3 i, \boldsymbol{\nu}} \leq \mathscr{B}_{4 i, \boldsymbol{\nu}}+\eta_{i}^{*}(\boldsymbol{\nu})$ as $\frac{\partial l(\ldots)}{\partial w_{3 i}^{*}(\boldsymbol{\nu})} \leq 0$ and $\mathscr{B}_{4 i, \boldsymbol{\nu}} \leq \mathscr{B}_{4 i, \boldsymbol{\nu}}+\eta_{i}^{*}(\boldsymbol{\nu})$ because $\eta_{i}^{*}(\boldsymbol{\nu}) \geq 0$ as $w_{4 i}^{*}(\boldsymbol{\nu})=1-w_{a}^{*}(\boldsymbol{\nu})-w_{3 i}^{*}(\boldsymbol{\nu})=0$.

- We then have the solution for $w_{3 i}^{*}(\boldsymbol{\nu})$ and $w_{4 i}^{*}(\boldsymbol{\nu})$ as follows.

$$
\begin{aligned}
& w_{3 i}^{*}(\boldsymbol{\nu})= \begin{cases}0, & 1-w_{a}^{*}(\boldsymbol{\nu})=0 \\
0, & 1-w_{a}^{*}(\boldsymbol{\nu})>0, \mathscr{B}_{3 i, \boldsymbol{\nu}} \leq \mathscr{B}_{4 i, \boldsymbol{\nu}} \\
\kappa_{3 i}^{*}(\boldsymbol{\nu}), & 1-w_{a}^{*}(\boldsymbol{\nu})>0, \mathscr{B}_{3 i, \boldsymbol{\nu}}=\mathscr{B}_{4 i, \boldsymbol{\nu}} \\
1-w_{a}^{*}(\boldsymbol{\nu}), & 1-w_{a}^{*}(\boldsymbol{\nu})>0, \mathscr{B}_{3 i, \boldsymbol{\nu}} \geq \mathscr{B}_{4 i, \boldsymbol{\nu}}\end{cases} \\
& w_{4 i}^{*}(\boldsymbol{\nu})=\left(1-w_{a}^{*}(\boldsymbol{\nu})\right)-w_{3 i}^{*}(\boldsymbol{\nu}) .
\end{aligned}
$$


where $0<\kappa_{3 i}^{*}(\boldsymbol{\nu})<1-w_{a}^{*}(\boldsymbol{\nu})$.

Optimal solutions for $w_{1 i}^{*}(\boldsymbol{\nu})$ and $w_{a}^{*}(\boldsymbol{\nu})$

From KKT condition (2.9),

$$
\begin{gathered}
w_{1 i}^{*}(\boldsymbol{\nu}) \begin{cases}>0, & \frac{\partial l(\ldots)}{\partial w_{1 i}^{*}(\boldsymbol{\nu})}=0 \\
=0, & \frac{\partial l(\ldots)}{\partial w_{1 i}^{*}(\boldsymbol{\nu})} \leq 0\end{cases} \\
\frac{\partial l(\ldots)}{\partial w_{1 i}^{*}(\boldsymbol{\nu})}=\left(s_{i}^{*}+\left(r_{s i}\left(\boldsymbol{\nu}, p_{1 i}^{*}(\boldsymbol{\nu})\right)-\lambda^{*} p_{1 i}^{*}(\boldsymbol{\nu})\right)+\frac{q^{*}(\boldsymbol{\nu})}{w_{a}^{*}(\boldsymbol{\nu})} \frac{p_{1 i}^{*}(\boldsymbol{\nu})-p_{2 i}^{*}(\boldsymbol{\nu})}{\frac{w_{1 i}^{*}(\boldsymbol{\nu})}{w_{a}^{*}(\boldsymbol{\nu})} p_{1 i}^{*}(\boldsymbol{\nu})+\frac{\left(w_{a}^{*}(\boldsymbol{\nu})-w_{1 i}^{*}(\boldsymbol{\nu})\right)}{w_{a}^{*}(\boldsymbol{\nu})} p_{2 i}^{*}(\boldsymbol{\nu})+\frac{\alpha_{i} P_{p i}(\boldsymbol{\nu})+N_{0}}{h_{i}}}\right) \\
\\
-\left(r_{s i}\left(\boldsymbol{\nu}, p_{2 i}^{*}(\boldsymbol{\nu})\right)-\lambda^{*} p_{2 i}^{*}(\boldsymbol{\nu})+\gamma_{i}^{*}(\boldsymbol{\nu})\right)
\end{gathered}
$$

Remark: Again, note that the term $u_{i}^{*}(\boldsymbol{\nu})\left(p_{r p, i}(\boldsymbol{\nu})-p_{1 i}^{*}\right)=0$ due to the optimality condition.

From KKT condition in $(2.8)$ that $\frac{\partial l(\ldots)}{\partial w_{1 i}^{*}(\boldsymbol{\nu})}=0$, we also have

$$
\begin{aligned}
& s_{a}^{*}+\sum_{i=1}^{N}\left(r_{s i}\left(\boldsymbol{\nu}, p_{2 i}^{*}(\boldsymbol{\nu})\right)-\lambda^{*} p_{2 i}^{*}(\boldsymbol{\nu})+\gamma_{i}^{*}(\boldsymbol{\nu})-\frac{q^{*}(\boldsymbol{\nu}) w_{1 i}^{*}(\boldsymbol{\nu})}{\left(w_{a}^{*}(\boldsymbol{\nu})\right)^{2}} \frac{p_{1 i}^{*}(\boldsymbol{\nu})-p_{2 i}^{*}(\boldsymbol{\nu})}{\frac{w_{1 i}^{*}(\boldsymbol{\nu})}{w_{a}^{*}(\boldsymbol{\nu})} p_{1 i}^{*}(\boldsymbol{\nu})+\frac{\left(w_{a}^{*}(\boldsymbol{\nu})-w_{i i}^{*}(\boldsymbol{\nu})\right)}{w_{a}^{*}(\boldsymbol{\nu})} p_{2 i}^{*}(\boldsymbol{\nu})+\frac{\alpha_{i} P_{p i}(\boldsymbol{\nu})+N_{0}}{h_{i}}}\right) \\
& =\sum_{i=1}^{N}\left(r_{s i}\left(\boldsymbol{\nu}, p_{4 i}^{*}(\boldsymbol{\nu})\right)-\lambda^{*} p_{4 i}^{*}(\boldsymbol{\nu})+\eta_{i}^{*}(\boldsymbol{\nu})\right)
\end{aligned}
$$

CASE 1: If $q^{*}(\boldsymbol{\nu})>0$

Since we have shown that in this case $w_{1 i}^{*}(\boldsymbol{\nu})$ and $w_{2 i}^{*}(\boldsymbol{\nu})$ cannot be positive at the same time (i.e. $\mathscr{S}_{1} \cap \mathscr{S}_{2}$ is an empty set), we have the two following cases:

CASE 1.1: $w_{1 i}^{*}(\boldsymbol{\nu})=w_{a}^{*}(\boldsymbol{\nu})>0$ and $w_{2 i}^{*}(\boldsymbol{\nu})=0 \quad \Rightarrow \gamma_{i}^{*}(\boldsymbol{\nu}) \geq 0$

We have $p_{1 i}^{*}(\boldsymbol{\nu})=p_{r s, i}(\boldsymbol{\nu})$ and the condition from $\frac{\partial l(\ldots)}{\partial w_{1 i}^{*}(\boldsymbol{\nu})}$ that

$$
\begin{aligned}
\mathscr{B}_{1 i, \boldsymbol{\nu}} & =\left(s_{i}^{*}+\left(r_{s i}\left(\boldsymbol{\nu}, p_{1 i}^{*}(\boldsymbol{\nu})\right)-\lambda^{*} p_{1 i}^{*}(\boldsymbol{\nu})\right)\right) \\
& =\left(r_{s i}\left(\boldsymbol{\nu}, p_{2 i}^{*}(\boldsymbol{\nu})\right)-\lambda^{*} p_{2 i}^{*}(\boldsymbol{\nu})+\gamma_{i}^{*}(\boldsymbol{\nu})-\frac{q^{*}(\boldsymbol{\nu})}{w_{a}^{*}(\boldsymbol{\nu})} \frac{p_{1 i}^{*}(\boldsymbol{\nu})-p_{2 i}^{*}(\boldsymbol{\nu})}{\frac{w_{1 i}^{*}(\boldsymbol{\nu})}{w_{a}^{*}(\boldsymbol{\nu})} p_{1 i}^{*}(\boldsymbol{\nu})+\frac{\left(w_{a}^{*}(\boldsymbol{\nu})-w_{1 i}^{*}(\boldsymbol{\nu})\right)}{w_{a}^{*}(\boldsymbol{\nu})} p_{2 i}^{*}(\boldsymbol{\nu})+\frac{\alpha_{i} P_{p i}(\boldsymbol{\nu})+N_{0}}{h_{i}}}\right) \\
& =\left(r_{s i}\left(\boldsymbol{\nu}, p_{2 i}^{*}(\boldsymbol{\nu})\right)-\lambda^{*} p_{2 i}^{*}(\boldsymbol{\nu})+\gamma_{i}^{*}(\boldsymbol{\nu})-\frac{q^{*}(\boldsymbol{\nu})}{w_{a}^{*}(\boldsymbol{\nu})} \frac{p_{1 i}^{*}(\boldsymbol{\nu})-p_{2 i}^{*}(\boldsymbol{\nu})+N_{0}}{p_{r s, i}(\boldsymbol{\nu})+\frac{\alpha_{i} P_{p i}(\boldsymbol{\nu})+N_{0}}{h_{i}}}\right)
\end{aligned}
$$

For $w_{2 i}^{*}(\boldsymbol{\nu})=0$, we can set $p_{2 i}^{*}(\boldsymbol{\nu})=p_{r s, i}^{*}(\boldsymbol{\nu})$ arbitrarily, resulting in $\mathscr{B}_{1 i, \boldsymbol{\nu}}=\mathscr{B}_{2 i, \boldsymbol{\nu}}+$ $\gamma_{i}^{*}(\boldsymbol{\nu}) \geq \mathscr{B}_{2 i, \boldsymbol{\nu}}$. Note that this case happens when $p_{r s, i}(\boldsymbol{\nu}) \leq p_{r p, i}(\boldsymbol{\nu})$, i.e. $i$-th PU is 
not disturbed by $p_{1 i}^{*}(\boldsymbol{\nu})=p_{r s, i}^{*}(\boldsymbol{\nu})$.

CASE 1.2: $w_{1 i}^{*}(\boldsymbol{\nu})=0$ and $w_{2 i}^{*}(\boldsymbol{\nu})=w_{a}^{*}(\boldsymbol{\nu})>0 \quad \Rightarrow \gamma_{i}^{*}(\boldsymbol{\nu})=0$

$$
\begin{aligned}
& \mathscr{B}_{1 i, \boldsymbol{\nu}}=\left(s_{i}^{*}+\left(r_{s i}\left(\boldsymbol{\nu}, p_{1 i}^{*}(\boldsymbol{\nu})\right)-\lambda^{*} p_{1 i}^{*}(\boldsymbol{\nu})\right)\right) \\
& \leq\left(r_{s i}\left(\boldsymbol{\nu}, p_{2 i}^{*}(\boldsymbol{\nu})\right)-\lambda^{*} p_{2 i}^{*}(\boldsymbol{\nu})+0-\frac{q^{*}(\boldsymbol{\nu})}{w_{a}^{*}(\boldsymbol{\nu})} \frac{p_{1 i}^{*}(\boldsymbol{\nu})-p_{2 i}^{*}(\boldsymbol{\nu})}{\frac{w_{1}^{*}(\boldsymbol{\nu})}{w_{a}^{*}(\boldsymbol{\nu})} p_{1 i}^{*}(\boldsymbol{\nu})+\frac{\left(w_{a}^{*}(\boldsymbol{\nu})-w_{1 i}^{*}(\boldsymbol{\nu})\right)}{w_{a}^{*}(\boldsymbol{\nu})} p_{2 i}^{*}(\boldsymbol{\nu})+\frac{\alpha_{i} P_{p i}(\boldsymbol{\nu})+N_{0}}{h_{i}}}\right) \\
& =\mathscr{B}_{2 i, \boldsymbol{\nu}}
\end{aligned}
$$

As $\left.p_{1 i}^{*}(\boldsymbol{\nu})\right)$ can be arbitrarily set to be equal to $p_{2 i}^{*}(\boldsymbol{\nu})$ since $w_{1 i}^{*}(\boldsymbol{\nu})=0$.

Note: For $w_{a}^{*}(\boldsymbol{\nu})>0$ and $q^{*}(\boldsymbol{\nu})>0$

Consider the term

$s_{a}^{*}+\sum_{i=1}^{N}\left(r_{s i}\left(\boldsymbol{\nu}, p_{2 i}^{*}(\boldsymbol{\nu})\right)-\lambda^{*} p_{2 i}^{*}(\boldsymbol{\nu})+\gamma_{i}^{*}(\boldsymbol{\nu})-\frac{q^{*}(\boldsymbol{\nu}) w_{1 i}^{*}(\boldsymbol{\nu})}{\left(w_{a}^{*}(\boldsymbol{\nu})\right)^{2}} \frac{p_{1 i}^{*}(\boldsymbol{\nu})-p_{2 i}^{*}(\boldsymbol{\nu})}{\frac{w_{1 i}^{*}(\boldsymbol{\nu})}{w_{a}^{*}(\boldsymbol{\nu})} p_{1 i}^{*}(\boldsymbol{\nu})+\frac{\left(w_{a}^{*}(\boldsymbol{\nu})-w_{1 i}^{*}(\boldsymbol{\nu})\right)}{w_{a}^{*}(\boldsymbol{\nu})} p_{2 i}^{*}(\boldsymbol{\nu})+\frac{\alpha_{i} P_{p i}(\boldsymbol{\nu})+N_{0}}{h_{i}}}\right)$.

Then, $r_{s i}\left(\boldsymbol{\nu}, p_{2 i}^{*}(\boldsymbol{\nu})\right)-\lambda^{*} p_{2 i}^{*}(\boldsymbol{\nu})+\gamma_{i}^{*}(\boldsymbol{\nu})-\frac{q^{*}(\boldsymbol{\nu}) w_{1 i}^{*}(\boldsymbol{\nu})}{\left(w_{a}^{*}(\boldsymbol{\nu})\right)^{2}} \frac{p_{1 i}^{*}(\boldsymbol{\nu})-p_{2 i}^{*}(\boldsymbol{\nu})}{\frac{w_{1 i}^{*}(\boldsymbol{\nu})}{w_{a}^{*}(\boldsymbol{\nu})} p_{1 i}^{*}(\boldsymbol{\nu})+\frac{\left(w_{a}^{*}(\boldsymbol{\nu})-w_{1 i}^{*}(\boldsymbol{\nu})\right)}{w_{a}^{*}(\boldsymbol{\nu})} p_{2 i}^{*}(\boldsymbol{\nu})+\frac{\alpha_{i} P_{p i}(\boldsymbol{\nu})+N_{0}}{h_{i}}}$ can be replaced by $\mathscr{B}_{1 i, \boldsymbol{\nu}}$ for the subchannel that $w_{1 i}^{*}(\boldsymbol{\nu})=w_{a}^{*}(\boldsymbol{\nu})$. Likewise, in the subchannel that $w_{2 i}^{*}(\boldsymbol{\nu})=w_{a}^{*}(\boldsymbol{\nu})$, we can replace the term by $\mathscr{B}_{2 i, \boldsymbol{\nu}}$ as $\gamma_{i}^{*}(\boldsymbol{\nu})=0$ and $\frac{q^{*}(\boldsymbol{\nu}) w_{1 i}^{*}(\boldsymbol{\nu})}{\left(w_{a}^{*}(\boldsymbol{\nu})\right)^{2}} \frac{p_{1 i}^{*}(\boldsymbol{\nu})-p_{2 i}^{*}(\boldsymbol{\nu})}{p_{r s, i}(\boldsymbol{\nu})+\frac{\alpha_{i} p_{i}(\boldsymbol{\nu})+N_{0}}{h_{i}}}=0$.

Thus, we can replace the left-hand side of (A.8) by $s_{a}^{*}+\sum_{i \in \mathscr{S}_{1}} \mathscr{B}_{1 i, \boldsymbol{\nu}}+\sum_{i \in \mathscr{S}_{2}} \mathscr{B}_{2 i, \boldsymbol{\nu}}-$ $\sum_{i \in \mathscr{S}_{1} \cap \mathscr{S}_{2}} \mathscr{B}_{1 i, \boldsymbol{\nu}}$, where $\sum_{i \in \mathscr{S}_{1} \cap \mathscr{S}_{2}} \mathscr{B}_{1 i, \boldsymbol{\nu}}$ appears when both $w_{1 i}^{*}(\boldsymbol{\nu})$ and $w_{2 i}^{*}(\boldsymbol{\nu})$ are positive. However, we have $\sum_{i \in \mathscr{S}_{1} \cap \mathscr{S}_{2}} \mathscr{B}_{1 i, \boldsymbol{\nu}}=0$ in this case because either $w_{1}^{*}(\boldsymbol{\nu})$ or $w_{2}^{*}(\boldsymbol{\nu})$ is positive in this case and $\mathscr{S}_{1} \cap \mathscr{S}_{2}$ is an empty set. Then, the following conditions are obtained.

- $0<w_{a}^{*}(\boldsymbol{\nu})<1$

In this case, both $w_{a}^{*}(\boldsymbol{\nu})$ and $1-w_{a}^{*}(\boldsymbol{\nu})$ are positive. We can also replace the right-hand side of (A.8) by $\sum_{i \in \mathscr{S}_{3}} \mathscr{B}_{3 i, \boldsymbol{\nu}}+\sum_{i \in \mathscr{S}_{4}} \mathscr{B}_{4 i, \boldsymbol{\nu}}-\sum_{i \in \mathscr{S}_{3} \cap \mathscr{S}_{4}} \mathscr{B}_{3 i, \boldsymbol{\nu}}$ as $\mathscr{B}_{4 i, \boldsymbol{\nu}}+\eta_{i}^{*}(\boldsymbol{\nu})$ is equal to either $\mathscr{B}_{3 i, \boldsymbol{\nu}}$ or $\mathscr{B}_{4 i, \boldsymbol{\nu}}$ as shown previously.

Finally, we have

$$
s_{a}^{*}+\sum_{i \in \mathscr{S}_{1}} \mathscr{B}_{1 i, \boldsymbol{\nu}}+\sum_{i \in \mathscr{S}_{2}} \mathscr{B}_{2 i, \boldsymbol{\nu}}-\sum_{i \in \mathscr{S}_{1} \cap \mathscr{S}_{2}} \mathscr{B}_{1 i, \boldsymbol{\nu}}=\sum_{i \in \mathscr{S}_{3}} \mathscr{B}_{3 i, \boldsymbol{\nu}}+\sum_{i \in \mathscr{S}_{4}} \mathscr{B}_{4 i, \boldsymbol{\nu}}-\sum_{i \in \mathscr{S}_{3} \cap \mathscr{S}_{4}} \mathscr{B}_{3 i, \boldsymbol{\nu}}
$$

Note that the intersection term appears if both $w_{3 i}^{*}(\boldsymbol{\nu})$ and $w_{4 i}^{*}(\boldsymbol{\nu})$ are positive at the same time, thereby yielding $\mathscr{B}_{3 i, \boldsymbol{\nu}}=\mathscr{B}_{4 i, \boldsymbol{\nu}}$.

- $w_{a}^{*}(\boldsymbol{\nu})=1$ 
The right-hand side of $\left(\right.$ A.8) can be substituted by $\sum_{i=1}^{N}\left[\mathscr{B}_{4 i, \boldsymbol{\nu}}+\eta_{i}^{*}(\boldsymbol{\nu})\right]$. Since $1-w_{a}^{*}(\boldsymbol{\nu})=w_{3 i}^{*}(\boldsymbol{\nu})=w_{4 i}^{*}(\boldsymbol{\nu})=0$ and $\eta_{i}^{*}(\boldsymbol{\nu}) \geq 0$, we then have

$$
\begin{aligned}
s_{a}^{*}+\sum_{i \in \mathscr{S}_{1}} \mathscr{B}_{1 i, \boldsymbol{\nu}}+\sum_{i \in \mathscr{S}_{2}} \mathscr{B}_{2 i, \boldsymbol{\nu}}-\sum_{i \in \mathscr{S}_{1} \cap \mathscr{S}_{2}} \mathscr{B}_{1 i, \boldsymbol{\nu}} & =\sum_{i=1}^{N}\left[\mathscr{B}_{4 i, \boldsymbol{\nu}}+\eta_{i}^{*}(\boldsymbol{\nu})\right] \\
& \stackrel{(e)}{\geq} \sum_{i \in \mathscr{S}_{3}} \mathscr{B}_{3 i, \boldsymbol{\nu}}+\sum_{i \in \mathscr{S}_{4}} \mathscr{B}_{4 i, \boldsymbol{\nu}}-\sum_{i \in \mathscr{\mathscr { S }}_{3} \cap \mathscr{S}_{4}} \mathscr{B}_{3 i, \boldsymbol{\nu}}
\end{aligned}
$$

Note that $(e)$ follows from $\mathscr{B}_{3 i, \boldsymbol{\nu}} \leq \mathscr{B}_{4 i, \boldsymbol{\nu}}+\eta_{i}^{*}(\boldsymbol{\nu})$ due to the associated KKT condition and $\mathscr{B}_{4 i, \boldsymbol{\nu}} \leq \mathscr{B}_{4 i, \boldsymbol{\nu}}+\eta_{i}^{*}(\boldsymbol{\nu})$ because $\eta_{i}^{*}(\boldsymbol{\nu}) \geq 0$.

Hence, for $q^{*}(\boldsymbol{\nu})>0$ and $w_{a}^{*}(\boldsymbol{\nu})>0$, we have

$$
\begin{aligned}
w_{1 i}^{*}(\boldsymbol{\nu})= \begin{cases}0, & w_{a}^{*}(\boldsymbol{\nu})>0, \mathscr{B}_{1 i, \boldsymbol{\nu}} \leq \mathscr{B}_{2 i, \boldsymbol{\nu}} \\
w_{a}^{*}(\boldsymbol{\nu}), & w_{a}^{*}(\boldsymbol{\nu})>0, \mathscr{B}_{1 i, \boldsymbol{\nu}} \geq \mathscr{B}_{2 i, \boldsymbol{\nu}}\end{cases} \\
w_{2 i}^{*}(\boldsymbol{\nu})=w_{a}^{*}(\boldsymbol{\nu})-w_{1 i}^{*}(\boldsymbol{\nu}) .
\end{aligned}
$$

CASE 1.3: $w_{1 i}^{*}(\boldsymbol{\nu})=w_{2 i}^{*}(\boldsymbol{\nu})=w_{a}^{*}(\boldsymbol{\nu})=0 \Rightarrow \gamma_{i}^{*}(\boldsymbol{\nu}) \geq 0$

This case happens when SU can achieve the target rate with equality $\left(q^{*}(\boldsymbol{\nu})>0\right)$ but it is not selected as it is not an optimal strategy. As both $w_{1 i}^{*}(\boldsymbol{\nu})$ and $w_{2 i}^{*}(\boldsymbol{\nu})$ are zero, we can arbitrarily set $p_{1 i}^{*}(\boldsymbol{\nu})=p_{2 i}^{*}(\boldsymbol{\nu})=o\left(w_{a}^{*}(\boldsymbol{\nu})\right)$, such that $\mathscr{B}_{1 i, \boldsymbol{\nu}}+$ $\frac{q^{*}(\boldsymbol{\nu})}{w_{a}^{*}(\boldsymbol{\nu})} \frac{p_{1 i}^{*}(\boldsymbol{\nu})-p_{2 i}^{*}(\boldsymbol{\nu})}{\frac{w_{1 i}^{*}(\boldsymbol{\nu})}{w_{a}^{*}(\boldsymbol{\nu})} p_{1 i}^{*}(\boldsymbol{\nu})+\frac{\left(w_{a}^{*}(\boldsymbol{\nu})-w_{1 i}^{*}(\boldsymbol{\nu})\right)}{w_{a}^{*}(\boldsymbol{\nu})} p_{2 i}^{*}(\boldsymbol{\nu})+\frac{\alpha_{i} P_{p i}(\boldsymbol{\nu})+N_{0}}{h_{i}}}=\mathscr{B}_{1 i, \boldsymbol{\nu}} \leq \mathscr{B}_{2 i, \boldsymbol{\nu}}+\gamma_{i}^{*}(\boldsymbol{\nu})$. The choice of $o\left(w_{a}^{*}(\boldsymbol{\nu})\right)$ is to ensure that the term involving $\left(p_{1 i}^{*}(\boldsymbol{\nu})-p_{2 i}^{*}(\boldsymbol{\nu})\right)$ in the numerator approaches 0 in the limit as $w_{a}^{*}(\boldsymbol{\nu}) \rightarrow 0$.

Finally, we can conclude that for $q^{*}(\boldsymbol{\nu})>0$

$$
\begin{aligned}
w_{1 i}^{*}(\boldsymbol{\nu})= \begin{cases}0, & w_{a}^{*}(\boldsymbol{\nu})=0 \\
0, & w_{a}^{*}(\boldsymbol{\nu})>0, \mathscr{B}_{1 i, \boldsymbol{\nu}} \leq \mathscr{B}_{2 i, \boldsymbol{\nu}} \\
w_{a}^{*}(\boldsymbol{\nu}), & w_{a}^{*}(\boldsymbol{\nu})>0, \mathscr{B}_{1 i, \boldsymbol{\nu}} \geq \mathscr{B}_{2 i, \boldsymbol{\nu}}\end{cases} \\
w_{2 i}^{*}(\boldsymbol{\nu})=w_{a}^{*}(\boldsymbol{\nu})-w_{1 i}^{*}(\boldsymbol{\nu}) .
\end{aligned}
$$




$$
w_{a}^{*}(\boldsymbol{\nu})=\left\{\begin{aligned}
0, \quad s_{a}^{*} & +\sum_{i \in \mathscr{S}_{1}} \mathscr{B}_{1 i, \boldsymbol{\nu}}+\sum_{i \in \mathscr{S}_{2}} \mathscr{B}_{2 i, \boldsymbol{\nu}}-\sum_{i \in \mathscr{S}_{1} \cap \mathscr{S}_{2}} \mathscr{B}_{1 i, \boldsymbol{\nu}} \\
& \leq \sum_{i \in \mathscr{S}_{3}} \mathscr{B}_{3 i, \boldsymbol{\nu}}+\sum_{i \in \mathscr{S}_{4}} \mathscr{B}_{4 i, \boldsymbol{\nu}}-\sum_{i \in \mathscr{S}_{3} \cap \mathscr{S}_{4}} \mathscr{B}_{3 i, \boldsymbol{\nu}} \\
\kappa_{a}^{*}(\boldsymbol{\nu}), s_{a}^{*} & +\sum_{i \in \mathscr{S}_{1}} \mathscr{B}_{1 i, \boldsymbol{\nu}}+\sum_{i \in \mathscr{S}_{2}} \mathscr{B}_{2 i, \boldsymbol{\nu}}-\sum_{i \in \mathscr{S}_{1} \cap \mathscr{S}_{2}} \mathscr{B}_{1 i, \boldsymbol{\nu}} \\
& =\sum_{i \in \mathscr{S}_{3}} \mathscr{B}_{3 i, \boldsymbol{\nu}}+\sum_{i \in \mathscr{S}_{4}} \mathscr{B}_{4 i, \boldsymbol{\nu}}-\sum_{i \in \mathscr{S}_{3} \cap \mathscr{S}_{4}} \mathscr{B}_{3 i, \boldsymbol{\nu}} \\
1, \quad s_{a}^{*} & +\sum_{i \in \mathscr{S}_{1}} \mathscr{B}_{1 i, \boldsymbol{\nu}}+\sum_{i \in \mathscr{S}_{2}} \mathscr{B}_{2 i, \boldsymbol{\nu}}-\sum_{i \in \mathscr{S}_{1} \cap \mathscr{S}_{2}} \mathscr{B}_{1 i, \boldsymbol{\nu}} \\
& \geq \sum_{i \in \mathscr{S}_{3}} \mathscr{B}_{3 i, \boldsymbol{\nu}}+\sum_{i \in \mathscr{S}_{4}} \mathscr{B}_{4 i, \boldsymbol{\nu}}-\sum_{i \in \mathscr{S}_{3} \cap \mathscr{S}_{4}} \mathscr{B}_{3 i, \boldsymbol{\nu}}
\end{aligned}\right.
$$

CASE 2: If $q^{*}(\boldsymbol{\nu})=0$

- Suppose that $w_{a}^{*}(\boldsymbol{\nu})>0$. From (A.8),

$$
\begin{aligned}
\frac{\partial l(\ldots)}{\partial w_{1 i}^{*}(\boldsymbol{\nu})}=0 & \Rightarrow w_{1 i}^{*}(\boldsymbol{\nu})>0, \mathscr{B}_{1 i, \boldsymbol{\nu}}=\mathscr{B}_{2 i, \boldsymbol{\nu}}+\gamma_{i}^{*} \geq \mathscr{B}_{2 i, \boldsymbol{\nu}} \\
& \Rightarrow\left\{\begin{array}{l}
w_{2 i}^{*}(\boldsymbol{\nu})=0, \quad \mathscr{B}_{1 i, \boldsymbol{\nu}} \geq \mathscr{B}_{2 i, \boldsymbol{\nu}}, \gamma_{i}^{*} \geq 0 \\
w_{2 i}^{*}(\boldsymbol{\nu})>0, \quad \mathscr{B}_{1 i, \boldsymbol{\nu}}=\mathscr{B}_{2 i, \boldsymbol{\nu}}, \gamma_{i}^{*}=0
\end{array}\right. \\
\frac{\partial l(\ldots)}{\partial w_{1 i}^{*}(\boldsymbol{\nu})} \leq 0 & \Rightarrow w_{1 i}^{*}=0, \mathscr{B}_{1 i, \boldsymbol{\nu}} \leq \mathscr{B}_{2 i, \boldsymbol{\nu}}+\gamma_{i}^{*}=\mathscr{B}_{2 i, \boldsymbol{\nu}}, \quad \text { as } \gamma_{i}^{*}=0
\end{aligned}
$$

- If $w_{a}^{*}(\boldsymbol{\nu})=0$, then $w_{1 i}^{*}(\boldsymbol{\nu})=w_{2 i}^{*}(\boldsymbol{\nu})=0$. Note that as $q^{*}(\boldsymbol{\nu})=0$, we then have $\mathscr{B}_{1 i, \boldsymbol{\nu}} \leq \mathscr{B}_{2 i, \boldsymbol{\nu}}+\gamma_{i}^{*}(\boldsymbol{\nu})$ by KKT condition and $\mathscr{B}_{2 i, \boldsymbol{\nu}} \leq \mathscr{B}_{2 i, \boldsymbol{\nu}}+\gamma_{i}^{*}(\boldsymbol{\nu})$ as $w_{2 i}^{*}(\boldsymbol{\nu})=0$, making $\gamma_{i}^{*}(\boldsymbol{\nu}) \geq 0$ for all $i$.

- We then have the solution for $w_{1 i}^{*}(\boldsymbol{\nu})$ and $w_{2 i}^{*}(\boldsymbol{\nu})$ for $q^{*}(\boldsymbol{\nu})=0$ as follows.

$$
\begin{gathered}
w_{1 i}^{*}(\boldsymbol{\nu})= \begin{cases}0, & w_{a}^{*}(\boldsymbol{\nu})=0 \\
0, & w_{a}^{*}(\boldsymbol{\nu})>0, \mathscr{B}_{1 i, \boldsymbol{\nu}} \leq \mathscr{B}_{2 i, \boldsymbol{\nu}} \\
\kappa_{1 i}^{*}(\boldsymbol{\nu}), & w_{a}^{*}(\boldsymbol{\nu})>0, \mathscr{B}_{1 i, \boldsymbol{\nu}}=\mathscr{B}_{2 i, \boldsymbol{\nu}} \\
w_{a}^{*}(\boldsymbol{\nu}), & w_{a}^{*}(\boldsymbol{\nu})>0, \mathscr{B}_{1 i, \boldsymbol{\nu}} \geq \mathscr{B}_{2 i, \boldsymbol{\nu}}\end{cases} \\
w_{2 i}^{*}(\boldsymbol{\nu})=w_{a}^{*}(\boldsymbol{\nu})-w_{1 i}^{*}(\boldsymbol{\nu}) .
\end{gathered}
$$

where $0<\kappa_{1 i}^{*}(\boldsymbol{\nu})<w_{a}^{*}(\boldsymbol{\nu})$.

Then, as $q^{*}(\boldsymbol{\nu})=0$, for every $\mathscr{B}_{2 i, \boldsymbol{\nu}}+\gamma_{i}^{*}(\boldsymbol{\nu})$, it can be replaced by either $\mathscr{B}_{1 i, \boldsymbol{\nu}}$ or $\mathscr{B}_{2 i, \nu}$.

There are 3 cases that we have to consider. 
1. $0<w_{a}^{*}(\boldsymbol{\nu})<1$

In this case, both $w_{a}^{*}(\boldsymbol{\nu})$ and $1-w_{a}^{*}(\boldsymbol{\nu})$ are positive and $q^{*}(\boldsymbol{\nu})=0$. Thus, we can replace the left-hand side of (A.8) by $s_{a}^{*}+\sum_{i \in \mathscr{S}_{1}} \mathscr{B}_{1 i, \boldsymbol{\nu}}+\sum_{i \in \mathscr{S}_{2}} \mathscr{B}_{2 i, \boldsymbol{\nu}}-\sum_{i \in \mathscr{S}_{1} \cap \mathscr{S}_{2}} \mathscr{B}_{1 i, \boldsymbol{\nu}}$ and the right-hand side by $\sum_{i \in \mathscr{S}_{3}} \mathscr{B}_{3 i, \boldsymbol{\nu}}+\sum_{i \in \mathscr{S}_{4}} \mathscr{B}_{4 i, \boldsymbol{\nu}}-\sum_{i \in \mathscr{S}_{3} \cap \mathscr{S}_{4}} \mathscr{B}_{3 i, \boldsymbol{\nu}}$. Finally, we have

$$
s_{a}^{*}+\sum_{i \in \mathscr{S}_{1}} \mathscr{B}_{1 i, \boldsymbol{\nu}}+\sum_{i \in \mathscr{S}_{2}} \mathscr{B}_{2 i, \boldsymbol{\nu}}-\sum_{i \in \mathscr{\mathscr { S }}_{1} \cap \mathscr{S}_{2}} \mathscr{B}_{1 i, \boldsymbol{\nu}}=\sum_{i \in \mathscr{S}_{3}} \mathscr{B}_{3 i, \boldsymbol{\nu}}+\sum_{i \in \mathscr{S}_{4}} \mathscr{B}_{4 i, \boldsymbol{\nu}}-\sum_{i \in \mathscr{S}_{3} \cap \mathscr{S}_{4}} \mathscr{B}_{3 i, \boldsymbol{\nu}}
$$

Again, note that the intersection term indicates the possibility of using a randomized strategy for a given channel state $\boldsymbol{\nu}$, which can happen when the fading state is discrete.

2. $w_{a}^{*}(\boldsymbol{\nu})=1$

As $w_{a}^{*}(\boldsymbol{\nu})>0$, the left-hand side of $\left(\right.$ A.8) can be replaced by $s_{a}^{*}+\sum_{i \in \mathscr{S}_{1}} \mathscr{B}_{1 i, \boldsymbol{\nu}}+$ $\sum_{i \in \mathscr{S}_{2}} \mathscr{B}_{2 i, \boldsymbol{\nu}}-\sum_{i \in \mathscr{S}_{1} \cap \mathscr{S}_{2}} \mathscr{B}_{1 i, \boldsymbol{\nu}}$ while $\sum_{i=1}^{N}\left[\mathscr{B}_{4 i, \boldsymbol{\nu}}+\eta_{i}^{*}(\boldsymbol{\nu})\right]$ can substitute the right-hand side of (A.8).

Since $1-w_{a}^{*}(\boldsymbol{\nu})=w_{3 i}^{*}(\boldsymbol{\nu})=w_{4 i}^{*}(\boldsymbol{\nu})=0$ and $\eta_{i}^{*}(\boldsymbol{\nu}) \geq 0$, we then have

$$
\begin{aligned}
s_{a}^{*}+\sum_{i \in \mathscr{S}_{1}} \mathscr{B}_{1 i, \boldsymbol{\nu}}+\sum_{i \in \mathscr{S}_{2}} \mathscr{B}_{2 i, \boldsymbol{\nu}}-\sum_{i \in \mathscr{\mathscr { S }}_{1} \cap \mathscr{S}_{2}} \mathscr{B}_{1 i, \boldsymbol{\nu}} & =\sum_{i=1}^{N}\left[\mathscr{B}_{4 i, \boldsymbol{\nu}}+\eta_{i}^{*}(\boldsymbol{\nu})\right] \\
& \stackrel{(g)}{\geq} \sum_{i \in \mathscr{S}_{3}} \mathscr{B}_{3 i, \boldsymbol{\nu}}+\sum_{i \in \mathscr{S}_{4}} \mathscr{B}_{4 i, \boldsymbol{\nu}}-\sum_{i \in \mathscr{S}_{3} \cap \mathscr{S}_{4}} \mathscr{B}_{3 i, \boldsymbol{\nu}}
\end{aligned}
$$

Note that $(g)$ follows from the fact that $\mathscr{B}_{3 i, \boldsymbol{\nu}} \leq \mathscr{B}_{4 i, \boldsymbol{\nu}}+\eta_{i}^{*}(\boldsymbol{\nu})$ due to the associated KKT condition and $\mathscr{B}_{4 i, \boldsymbol{\nu}} \leq \mathscr{B}_{4 i, \boldsymbol{\nu}}+\eta_{i}^{*}(\boldsymbol{\nu})$ since $\eta_{i}^{*}(\boldsymbol{\nu}) \geq 0$ for all $i$.

3. $w_{a}^{*}(\boldsymbol{\nu})=0$

As $1-w_{a}^{*}(\boldsymbol{\nu})>0$, the right-hand side of (A.8) becomes $\sum_{i \in \mathscr{S}_{3}} \mathscr{B}_{3 i, \boldsymbol{\nu}}+\sum_{i \in \mathscr{S}_{4}} \mathscr{B}_{4 i, \boldsymbol{\nu}}-$ $\sum_{i \in \mathscr{S}_{3} \cap \mathscr{S}_{4}} \mathscr{B}_{3 i, \boldsymbol{\nu}}$ and the left-hand side is $s_{a}^{*}+\sum_{i=1}^{N}\left[\mathscr{B}_{2 i, \boldsymbol{\nu}}+\gamma_{i}^{*}(\boldsymbol{\nu})-0\right]$ because $q^{*}(\boldsymbol{\nu})=$ 0 . 
Since $w_{a}^{*}(\boldsymbol{\nu})=w_{1 i}^{*}(\boldsymbol{\nu})=w_{2 i}^{*}(\boldsymbol{\nu})=0$ and $\gamma_{i}^{*}(\boldsymbol{\nu}) \geq 0$, we have

$$
\begin{aligned}
\sum_{i \in \mathscr{S}_{3}} \mathscr{B}_{3 i, \boldsymbol{\nu}}+\sum_{i \in \mathscr{S}_{4}} \mathscr{B}_{4 i, \boldsymbol{\nu}}-\sum_{i \in \mathscr{S}_{3} \cap \mathscr{S}_{4}} \mathscr{B}_{3 i, \boldsymbol{\nu}} & =s_{a}^{*}+\sum_{i=1}^{N}\left[\mathscr{B}_{2 i, \boldsymbol{\nu}}+\gamma_{i}^{*}(\boldsymbol{\nu})-0\right] \\
& \stackrel{(h)}{\geq} s_{a}^{*}+\sum_{i \in \mathscr{S}_{1}} \mathscr{B}_{1 i, \boldsymbol{\nu}}+\sum_{i \in \mathscr{S}_{2}} \mathscr{B}_{2 i, \boldsymbol{\nu}}-\sum_{i \in \mathscr{S}_{1} \cap \mathscr{S}_{2}} \mathscr{B}_{1 i, \boldsymbol{\nu}}
\end{aligned}
$$

Note that $(h)$ follows from $\mathscr{B}_{1 i, \boldsymbol{\nu}} \leq \mathscr{B}_{2 i, \boldsymbol{\nu}}+\gamma_{i}^{*}(\boldsymbol{\nu})$ due to the associated KKT condition and $\mathscr{B}_{2 i, \boldsymbol{\nu}} \leq \mathscr{B}_{2 i, \boldsymbol{\nu}}+\gamma_{i}^{*}(\boldsymbol{\nu})$ since $\gamma_{i}^{*}(\boldsymbol{\nu})$ is non-negative.

Thus in this case also, we have

$$
w_{a}^{*}(\boldsymbol{\nu})=\left\{\begin{aligned}
0, & s_{a}^{*}+\sum_{i \in \mathscr{S}_{1}} \mathscr{B}_{1 i, \boldsymbol{\nu}}+\sum_{i \in \mathscr{S}_{2}} \mathscr{B}_{2 i, \boldsymbol{\nu}}-\sum_{i \in \mathscr{S}_{1} \cap \mathscr{S}_{2}} \mathscr{B}_{1 i, \boldsymbol{\nu}} \\
& \leq \sum_{i \in \mathscr{S}_{3}} \mathscr{B}_{3 i, \boldsymbol{\nu}}+\sum_{i \in \mathscr{S}_{4}} \mathscr{B}_{4 i, \boldsymbol{\nu}}-\sum_{i \in \mathscr{S}_{3} \cap \mathscr{S}_{4}} \mathscr{B}_{3 i, \boldsymbol{\nu}} \\
\kappa_{a}^{*}(\boldsymbol{\nu}), s_{a}^{*} & +\sum_{i \in \mathscr{S}_{1}} \mathscr{B}_{1 i, \boldsymbol{\nu}}+\sum_{i \in \mathscr{S}_{2}} \mathscr{B}_{2 i, \boldsymbol{\nu}}-\sum_{i \in \mathscr{S}_{1} \cap \mathscr{S}_{2}} \mathscr{B}_{1 i, \boldsymbol{\nu}} \\
& =\sum_{i \in \mathscr{S}_{3}} \mathscr{B}_{3 i, \boldsymbol{\nu}}+\sum_{i \in \mathscr{S}_{4}} \mathscr{B}_{4 i, \boldsymbol{\nu}}-\sum_{i \in \mathscr{S}_{3} \cap \mathscr{S}_{4}} \mathscr{B}_{3 i, \boldsymbol{\nu}} \\
s_{a}^{*} & +\sum_{i \in \mathscr{S}_{1}} \mathscr{B}_{1 i, \boldsymbol{\nu}}+\sum_{i \in \mathscr{S}_{2}} \mathscr{B}_{2 i, \boldsymbol{\nu}}-\sum_{i \in \mathscr{S}_{1} \cap \mathscr{S}_{2}} \mathscr{B}_{1 i, \boldsymbol{\nu}} \\
& \geq \sum_{i \in \mathscr{S}_{3}} \mathscr{B}_{3 i, \boldsymbol{\nu}}+\sum_{i \in \mathscr{S}_{4}} \mathscr{B}_{4 i, \boldsymbol{\nu}}-\sum_{i \in \mathscr{S}_{3} \cap \mathscr{S}_{4}} \mathscr{B}_{3 i, \boldsymbol{\nu}}
\end{aligned}\right.
$$

Therefore, (A.12) always holds regardless of the value of $q^{*}(\boldsymbol{\nu})$.

Remark: For the continuous fading case, the condition $s_{a}^{*}+\sum_{i \in \mathscr{H}_{1}} \mathscr{B}_{1 i, \boldsymbol{\nu}}+\sum_{i \in \mathscr{S}_{2}} \mathscr{B}_{2 i, \boldsymbol{\nu}}-$ $\sum_{i \in \mathscr{S}_{1} \cap \mathscr{S}_{2}} \mathscr{B}_{1 i, \boldsymbol{\nu}}=\sum_{i \in \mathscr{S}_{3}} \mathscr{B}_{3 i, \boldsymbol{\nu}}+\sum_{i \in \mathscr{S}_{4}} \mathscr{B}_{4 i, \boldsymbol{\nu}}-\sum_{i \in \mathscr{S}_{3} \cap \mathscr{S}_{4}} \mathscr{B}_{3 i, \boldsymbol{\nu}}$ is satisfied with probability of zero measure. In other words, we can simplify (A.12) to the following:

$$
w_{a}^{*}(\boldsymbol{\nu})=\left\{\begin{array}{cc}
0, & s_{a}^{*}+\sum_{i \in \mathscr{S}_{1}} \mathscr{B}_{1 i, \boldsymbol{\nu}}+\sum_{i \in \mathscr{S}_{2}} \mathscr{B}_{2 i, \boldsymbol{\nu}}-\sum_{i \in \mathscr{S}_{1} \cap \mathscr{S}_{2}} \mathscr{B}_{1 i, \boldsymbol{\nu}}<\sum_{i \in \mathscr{S}_{3}} \mathscr{B}_{3 i, \boldsymbol{\nu}}+\sum_{i \in \mathscr{S}_{4}} \mathscr{B}_{4 i, \boldsymbol{\nu}} \\
1, & s_{a}^{*}+\sum_{i \in \mathscr{S}_{1}} \mathscr{B}_{1 i, \boldsymbol{\nu}}+\sum_{i \in \mathscr{S}_{2}} \mathscr{B}_{2 i, \boldsymbol{\nu}}-\sum_{i \in \mathscr{S}_{1} \cap \mathscr{S}_{2}} \mathscr{B}_{1 i, \boldsymbol{\nu}}>\sum_{i \in \mathscr{S}_{3}} \mathscr{B}_{3 i, \boldsymbol{\nu}}+\sum_{i \in \mathscr{S}_{4}} \mathscr{B}_{4 i, \boldsymbol{\nu}}
\end{array}\right.
$$




\section{Appendix B}

\section{Proofs in Chapter 3}

\section{B.1 Proof of Lemma 3.2.1}

Proof. For an arbitrary feasible probabilistic power scheme $\mathbf{P}(\chi)$ with conditional $\operatorname{PDF} f_{\mathbf{P} \mid \chi}(\mathbf{p}(\chi) \mid \chi)$, another feasible scheme $\mathbf{P}_{s}^{\prime}(\chi)$, which is randomised between two deterministic power schemes with time-sharing factors $w(\chi)$, can achieve higher SU average rate. The feasibility of $\mathbf{P}(\chi)$ implies that $E\left[\sum_{i=1}^{M} P_{i}(\chi)\right] \leq P_{a v}$ and $\operatorname{Pr}\left\{r_{p}((\chi), \mathbf{P}(\chi))<r_{p}^{0}\right\} \leq \epsilon_{p}$.

Since $\mathbf{p}_{1}$ is feasible, we know that $r_{p}\left(\boldsymbol{\chi}, \mathbf{p}_{1}(\boldsymbol{\chi})\right) \geq E\left[r_{p}\left(\boldsymbol{\chi}, \mathbf{P}_{s}(\boldsymbol{\chi})\right) \mid r_{p}(\boldsymbol{\chi}, \mathbf{p}(\boldsymbol{\chi})) \geq\right.$ $\left.r_{p}^{0}, \boldsymbol{\chi}\right]$. Therefore, all of possible $\mathbf{p}(\boldsymbol{\chi})$ such that $r_{p}(\boldsymbol{\chi}, \mathbf{p}(\boldsymbol{\chi})) \geq r_{p}^{0}$ lie in the halfspace defined by $\mathbf{p}^{T}(\boldsymbol{\chi}) \boldsymbol{\beta} \leq\left(\frac{g P_{p}(g)}{e^{r 0}-1}-N_{0}\right)^{+}$. Thus, $\mathbf{p}_{1}(\boldsymbol{\chi})$ must also be in that halfspace, i.e. $\mathbf{p}_{1}^{T}(\chi) \boldsymbol{\beta} \leq\left(\frac{g P_{p}(g)}{e^{r p}-1}-N_{0}\right)^{+}$.

Construct the new probabilistic scheme $\mathbf{P}^{\prime}$ such that $\mathbf{P}^{\prime}=\mathbf{p}_{1}(\chi)$ with probability $w(\boldsymbol{\chi})$ and $\mathbf{P}^{\prime}=\mathbf{p}_{2}(\boldsymbol{\chi})$ with probability $1-w(\boldsymbol{\chi})$, where $w(\boldsymbol{\chi})=\operatorname{Pr}\left\{r_{p}(\boldsymbol{\chi}, \mathbf{P}(\boldsymbol{\chi})) \geq r_{p}^{0} \mid \boldsymbol{\chi}\right\}$.

For the PU's outage probability based on the policy $\mathbf{P}^{\prime}$, we can show that $\operatorname{Pr}\left\{r_{p}\left(\boldsymbol{\chi}, \mathbf{P}^{\prime}(\chi)\right) \geq r_{p}^{0} \mid \chi\right\} \geq w(\chi)$, so $E[w(\chi)] \geq 1-\epsilon_{p}$.

As $E\left[\sum_{i=1}^{M} w(\boldsymbol{\chi}) \mathbf{p}_{1}(\boldsymbol{\chi})+(1-w(\boldsymbol{\chi})) \mathbf{p}_{2}(\boldsymbol{\chi})\right]=E\left[\sum_{i=1}^{M} \mathbf{P}(\boldsymbol{\chi})\right] \preceq P_{a v}$, so the new power control $\mathbf{P}^{\prime}(\boldsymbol{\chi})$ satisfies the average transmit power constraint.

Finally, we can show that average SU rate by $\mathbf{P}^{\prime}(\chi)$,

$$
\begin{aligned}
& E\left[r_{s}\left(\boldsymbol{\chi}, \mathbf{P}^{\prime}(\boldsymbol{\chi})\right)\right] \\
& =E\left[w(\boldsymbol{\chi}) r_{s}\left(\boldsymbol{\chi}, \mathbf{p}_{1}(\boldsymbol{\chi})\right)+(1-w(\boldsymbol{\chi})) r_{s}\left(\boldsymbol{\chi}, \mathbf{p}_{2}(\boldsymbol{\chi})\right)\right] \\
& \geq E\left[r_{s}(\boldsymbol{\chi}, \mathbf{P}(\boldsymbol{\chi}))\right]
\end{aligned}
$$

by the aid of Jensen's inequality for concave function. 


\section{B.2 KKT conditions for ATPC}

$$
\begin{gathered}
\frac{\partial l(\ldots)}{\partial p_{k i}^{*}(\boldsymbol{\chi})}\left\{\begin{array}{l}
=0, \quad p_{k i}^{*}(\boldsymbol{\chi})>0 \\
\leq 0, \quad p_{k i}^{*}(\boldsymbol{\chi})=0
\end{array}\right. \\
\frac{\partial l(\ldots)}{\partial w^{*}(\boldsymbol{\chi})} \begin{cases}=0, & 0<w^{*}(\boldsymbol{\chi})<1 \\
\leq 0, & w^{*}(\boldsymbol{\chi})=0 \\
\geq 0, & w^{*}(\boldsymbol{\chi})=1\end{cases} \\
\Lambda^{*}\left(P_{a v}-E\left[\mathbf{1}^{T}\left(w(\boldsymbol{\chi}) \mathbf{p}_{1}(\boldsymbol{\chi})+(1-w(\boldsymbol{\chi})) \mathbf{p}_{2}(\boldsymbol{\chi})\right)\right]\right)=0 \\
S^{*}\left(E\left[w^{*}(\boldsymbol{\chi})\right]-\left(1-\epsilon_{p}\right)\right)=0 \\
U^{*}(\boldsymbol{\chi}) w^{*}(\boldsymbol{\chi})\left[\mathscr{P}_{p}(\boldsymbol{\chi})-\beta \mathbf{1}^{T} \mathbf{p}_{1}^{*}(\boldsymbol{\chi})\right]=0 \\
\mathbf{p}_{k}^{*}(\boldsymbol{\chi}) \succeq 0 \quad, \quad k=1,2 \\
0 \leq w^{*}(\boldsymbol{\chi}) \leq 1
\end{gathered}
$$

\section{B.3 Proof of Theorem 3.2.1}

we will derive the optimal solutions for $\mathbf{p}_{1}^{*}(\chi), \mathbf{p}_{2}^{*}(\chi)$, and $w^{*}(\chi)$ by using the conditions from (B.2) to (B.8). If $w^{*}(\chi)>0$, we have $\left(\frac{1}{1+\sum_{i=1}^{M} p_{1 i}^{*}(\chi) z_{i}} z_{i}-\Lambda^{*}-U^{*}(\chi) \beta\right) \leq$ 0 by (B.2) where the condition is met with equality iff $p_{1 i}^{*}(\chi)>0$. For any $j \neq i$, if $p_{1 i}^{*}(\boldsymbol{\chi})>0$ and $p_{1 j}^{*}(\chi)>0$, the condition $\frac{\Lambda^{*}+U^{*}(\boldsymbol{\chi}) \beta}{z_{i}}=\frac{\Lambda^{*}+U^{*}(\boldsymbol{\chi}) \beta}{z_{j}}$ must be satisfied. Notice that the numerator terms are exactly the same and $z_{i}$ is independent of $z_{j}$ which happens with zero probability for continuous fading distributions. Define $p_{R P}^{*}(\boldsymbol{\chi})=\frac{\mathscr{P}_{p}(\boldsymbol{\chi})}{\beta}$. Thus,

$$
p_{1 i}^{*}(\chi)= \begin{cases}p_{W F, i}^{*}(\chi) & , w^{*}(\chi)=1, p_{W F}^{*}(\chi) \leq p_{R P}^{*}(\chi), i=\arg \max _{m \in \mathcal{I}} z_{m} \\ p_{R P}^{*}(\chi) & , w^{*}(\chi)=1, p_{W F}^{*}(\chi)>p_{R P}^{*}(\chi), i=\arg \max _{m \in \mathcal{I}} z_{m} \\ 0 & , \text { otherwise }\end{cases}
$$


If $\left(1-w^{*}(\boldsymbol{\chi})\right)>0$, we can solve for $\mathbf{p}_{2 i}^{*}$ by (B.2), yielding $\left(\frac{1}{1+\sum_{i=1}^{M} p_{2 i}^{*}(\boldsymbol{\chi}) z_{i}} z_{i}-\Lambda^{*}\right) \leq 0$. The condition is satisfied with equality iff $p_{2 i}^{*}(\chi)>0$. Again, $\frac{z_{i}}{\Lambda^{*}}=\frac{z_{j}}{\Lambda^{*}}$ happens with zero probability for $i \neq j$ in the case of continuous fading channels. Therefore

$$
p_{2 i}^{*}(\chi)= \begin{cases}p_{W F, i}^{*}(\chi) & , w^{*}(\chi)=0, i=\arg \max _{m \in \mathcal{I}} z_{m} \\ 0 & , \text { otherwise }\end{cases}
$$

For $w^{*}(\chi)$, first define two benefit functions as follows.

$$
\begin{aligned}
& \mathscr{B}_{1, \boldsymbol{\chi}}^{B} \triangleq r_{s}\left(\boldsymbol{\chi}, \mathbf{p}_{1}^{*}(\boldsymbol{\chi})\right)-\Lambda^{*} \mathbf{1}^{T} \mathbf{p}_{1}^{*}(\boldsymbol{\chi})+S^{*} \\
& \mathscr{B}_{2, \boldsymbol{\chi}}^{B} \triangleq r_{s}\left(\boldsymbol{\chi}, \mathbf{p}_{2}^{*}(\boldsymbol{\chi})\right)-\Lambda^{*} \mathbf{1}^{T} \mathbf{p}_{2}^{*}(\boldsymbol{\chi})
\end{aligned}
$$

By (B.3), we get

$$
w^{*}(\chi)= \begin{cases}1, & \mathscr{B}_{1, \chi}^{B}>\mathscr{B}_{2, \chi}^{B} \\ 0, & \mathscr{B}_{1, \chi}^{B}<\mathscr{B}_{2, \boldsymbol{\chi}}^{B}\end{cases}
$$

\section{B.4 KKT conditions for PTPC}

$$
\begin{gathered}
\frac{\partial l(\ldots)}{\partial p_{k i}^{*}(\boldsymbol{\chi})} \begin{cases}=0, & p_{k i}^{*}(\boldsymbol{\chi})>0 \\
\leq 0, & p_{k i}^{*}(\boldsymbol{\chi})=0\end{cases} \\
\frac{\partial l(\ldots)}{\partial w^{*}(\boldsymbol{\chi})} \begin{cases}=0, & 0<w^{*}(\boldsymbol{\chi})<1 \\
\leq 0, & w^{*}(\boldsymbol{\chi})=0 \\
\geq 0, & w^{*}(\boldsymbol{\chi})=1\end{cases} \\
\Lambda^{*}(\boldsymbol{\chi})\left(P_{O}-\mathbf{1}^{T}\left(w(\boldsymbol{\chi}) \mathbf{p}_{1}(\boldsymbol{\chi})+(1-w(\boldsymbol{\chi})) \mathbf{p}_{2}(\boldsymbol{\chi})\right)\right)=0 \\
S^{*}\left(E\left[w^{*}(\boldsymbol{\chi})\right]-\left(1-\epsilon_{p}\right)\right)=0 \\
U^{*}(\boldsymbol{\chi}) w^{*}(\boldsymbol{\chi})\left[\mathscr{P}_{p}(\boldsymbol{\chi})-\beta \mathbf{1}^{T} \mathbf{p}_{1}^{*}(\boldsymbol{\chi})\right]=0 \\
\mathbf{p}_{k}^{*}(\boldsymbol{\chi}) \succeq 0 \quad, \quad k=1,2 \\
0 \leq w^{*}(\boldsymbol{\chi}) \leq 1 .
\end{gathered}
$$




\section{B.5 Proof of Theorem 3.2.2}

we will derive the optimal solutions for $\mathbf{p}_{1}^{*}(\chi), \mathbf{p}_{2}^{*}(\chi)$, and $w^{*}(\chi)$ by using the conditions from (B.13) to (B.19).

By applying the same procedure as in ATPC case, we can obtain the optimal power control in PTPC case. To avoid repetition, some crucial results in PTPC case are summarized as follows.

- When $1-w^{*}(\boldsymbol{\chi})>0$, the condition $\left(\frac{1}{1+\sum_{i=1}^{M} p_{2 i}^{*}(\chi) z_{i}} z_{i}-\Lambda^{*}(\boldsymbol{\chi})-U^{*}(\boldsymbol{\chi}) \beta\right) \leq 0$ can be acquired from KKT condition. Notice that $\Lambda^{*}=\Lambda^{*}(\chi)$ in this case because it is the Lagrange multiplier PTPC. The power control $\mathbf{p}_{2}^{*}$ is

$$
p_{2 i}^{*}(\chi)= \begin{cases}P_{O} & , w^{*}(\chi)=0, i=\arg \max _{m \in \mathcal{I}} z_{m} \\ 0 & , \text { otherwise }\end{cases}
$$

- When $w^{*}(\boldsymbol{\chi})>0$, it requires that $\left(\frac{1}{1+\sum_{i=1}^{M} p_{2 i}^{*}(\boldsymbol{\chi}) z_{i}} z_{i}-\Lambda^{*}(\boldsymbol{\chi})\right) \leq 0$. The power control $\mathbf{p}_{1}^{*}$ is

$$
p_{1 i}^{*}(\chi)= \begin{cases}P_{O} & , w^{*}(\chi)=1, P_{O} \leq p_{R P}^{*}(\chi), i=\arg \max _{m \in \mathcal{I}} z_{m} \\ p_{R P}^{*}(\chi) & , w^{*}(\boldsymbol{\chi})=1, P_{O}>p_{R P}^{*}(\boldsymbol{\chi}), i=\arg \max _{m \in \mathcal{I}} z_{m} \\ 0 & , \text { otherwise }\end{cases}
$$

- The solution for $w^{*}(\chi)$ in PTPC has the same expression as in (B.12) except $\Lambda^{*}$ is replaced by $\Lambda^{*}(\chi)$, i.e. it varies with channel state $\chi$.

\section{B.6 Proof of Lemma 3.3.1}

We know that $F_{Z}\left(z \mid \mathcal{S}_{1}^{c}\right)=1-\frac{\exp \left(-N_{0} z\right)}{1+P_{c} z}$ while $F_{Z}\left(z \mid \mathcal{S}_{1}\right)=1-\exp \left(-N_{0} z\right)$. Hence, we will prove $\lim _{M \rightarrow \infty} \frac{E\left[\log \left(z_{\max }\right) 1_{\left\{z_{\max } \geq \Lambda^{*}\right\}} \mid \mathcal{S}_{1}^{c}\right]}{\log (\log (M))}=1$ only due to the similarity in the procedure to prove that $\lim _{M \rightarrow \infty} \frac{E\left[\log \left(z_{\max }\right) 1_{\left\{z_{\max } \geq \Lambda^{*}\right\}} \mid \mathcal{S}_{1}\right]}{\log (\log (M))}=1$. 
The normalizing constants $a_{M}$ and $b_{M}$ for Type-I convergence can be determined by solving for $F\left(b_{M}\right)=1-\frac{1}{M}$ and $a_{M}=\psi\left(b_{M}\right)$, where $\psi$ is the reciprocal hazard function, i.e. $\psi(z)=\left(1-F_{Z}(z)\right) / f_{Z}(z)$.

Afterwards, we can apply recursive method to find $b_{M}$ and $a_{M}$ as follows.

$$
\begin{aligned}
b_{M} & =\frac{1}{N_{0}}\left[\log M-\log \left(1+P_{c} b_{M}\right)\right] \\
& =\frac{1}{N_{0}} \log M-\frac{1}{N_{0}} \log \left(1+\frac{P_{c}}{N_{0}}\left[\log M-\log \left(1+P_{c} b_{M}\right)\right]\right) \\
& =\frac{1}{N_{0}} \log M-\frac{1}{N_{0}} \log \left(\frac{P_{c}}{N_{0}} \log M\right)-\frac{1}{N_{0}} \log \left[1+\frac{1}{\frac{P_{c}}{N_{0}} \log M}-\frac{\log \left(1+P_{c} b_{M}\right)}{\frac{P_{c}}{N_{0}} \log M}\right] \\
& =\frac{1}{N_{0}} \log M-\frac{1}{N_{0}} \log \left(\frac{P_{c}}{N_{0}} \log M\right)+\frac{1}{N_{0}} \log \left[\frac{P_{c} \log M+1-\log \left(1+P_{c} b_{M}\right)}{N_{0}}\right] \\
& =\frac{1}{N_{0}} \log M-\frac{1}{N_{0}} \log \left(\frac{P_{c}}{N_{0}} \log M\right)+O\left(\frac{\log \log (M)}{\log (M)}\right) \\
a_{M} & =\frac{1-F_{X}\left(b_{M}\right)}{f_{X}\left(b_{M}\right)} \quad=\left(\frac{\exp \left(-N_{0} b_{M}\right)}{1+P_{c} b_{M}}\right) /\left(\frac{\exp \left(-N_{0} b_{M}\right)}{1+P_{c} b_{M}}\left[\frac{P_{c}}{1+P_{c} b_{M}}+N_{0}\right]\right) \\
& =\frac{P_{c} b_{M}+1}{N_{0} P_{c} b_{M}+N_{0}+P_{c}}=\frac{1}{N_{0}}\left(1-\frac{P_{c}}{N_{0}+P_{c}+N_{0} P_{c} b_{M}}\right) \\
& =\frac{1}{N_{0}}\left(1-\frac{P_{c}}{\xi}\right) \quad=\frac{1}{N_{0}}\left(1-O\left(\frac{1}{\log M}\right)\right)
\end{aligned}
$$

where $\xi=N_{0}+P_{c}+N_{0} P_{c}\left[\frac{1}{N_{0}} \log M-\frac{1}{N_{0}} \log \left(\frac{P_{c}}{N_{0}} \log M\right)+O\left(\frac{\log \log (M)}{\log (M)}\right)\right]$. Follow the same procedure as shown in [81], we can first show that, for given $g \geq g_{T}$, $\frac{\log \left(z_{\max }\right)}{\log (\log M)}$ converges in probability to 1 . Then, define the event $A$.

$$
A=\left\{\log M-\Lambda^{*} O(\log (\log M)) \leq z_{\max } \leq \log M-\Lambda^{*} O(1)\right\}
$$

For $M$ is large enough, we can then show that $\liminf _{M \rightarrow \infty} \frac{E\left[\log \left(z_{\max }\right) 1_{\left\{z_{\max } \geq \Lambda^{*}\right\}} \mid \mathcal{S}_{1}^{c}\right]}{\log (\log M)} \geq 1$ as follows.

$$
\begin{aligned}
& E\left[\log \left(z_{\max }\right) 1_{\left\{z_{\max } \geq \Lambda^{*}\right\}} \mid \mathcal{S}_{1}^{c}\right] \geq E\left[\log \left(z_{\max }\right) 1_{A} \mid \mathcal{S}_{1}^{c}\right] \geq \log (\log M)+O(1) \\
& \therefore \frac{E\left[\log \left(z_{\max }\right) 1_{\left\{z_{\max } \geq \Lambda^{*}\right\}} \mid \mathcal{S}_{1}^{c}\right]}{\log (\log M)} \geq \frac{E\left[\log \left(z_{\max }\right) 1_{A} \mid \mathcal{S}_{1}^{c}\right]}{\log (\log M)} \quad=1+o(1)
\end{aligned}
$$

Then, we can prove that $\lim _{M \rightarrow \infty} \frac{E\left[z_{\max } \mid \mathcal{S}_{1}^{c}\right]}{\log M}=1$, by similar procedure from the proof of Lemma 2 in [81]. Finally, $\lim \sup _{M \rightarrow \infty} \frac{E\left[\log \left(z_{\max }\right) 1_{\left\{z_{\max } \geq \Lambda^{*}\right\}} \mid \mathcal{S}_{1}^{c}\right]}{\log (\log M)} \leq 1$ can be shown as 
follows

$$
\begin{aligned}
\because E\left[\log \left(1+z_{\max }\right) \mid \mathcal{S}_{1}^{c}\right] & \leq E\left[\log \left(1+z_{\max }\right) \mid \mathcal{S}_{1}^{c}\right] \\
& \leq \log \left(1+E\left[z_{\max } \mid \mathcal{S}_{1}^{c}\right]\right. \\
& =\log (\log M)+O(1) \\
\therefore \quad \frac{E\left[\log \left(z_{\max }\right) 1_{\left\{z_{\max } \geq \Lambda^{*}\right\}} \mid \mathcal{S}_{1}^{c}\right]}{\log (\log M)} & =1+o(1)
\end{aligned}
$$

Note that the second inequality in (B.26) follows from Jensen's inequality and the equality is due to $\lim _{M \rightarrow \infty} \frac{E\left[z_{\max } \mid \mathcal{S}_{1}^{c}\right]}{\log M}=1$. By (B.25) and (B.26), we finally have $\lim _{M \rightarrow \infty} \frac{E\left[\log \left(z_{\max }\right) 1_{\left\{z_{\max } \geq \Lambda^{*}\right\}} \mid \mathcal{S}_{1}^{c}\right]}{\log (\log M)}=1$.

\section{B.7 Proof of Lemma 3.3.2}

First, we will investigate the bound of $\Lambda^{*}$. As ATPC is always met, we have

$$
\begin{aligned}
P_{a v} & =\sum_{k=1}^{3} \operatorname{Pr}\left(\mathcal{S}_{k}\right) E\left[\left(\frac{1}{\Lambda^{*}}-\frac{1}{z_{\max }}\right)^{+} \mid \mathcal{S}_{k}\right]+\operatorname{Pr}\left(\mathcal{S}_{4}\right) E\left[\left(\frac{1}{\Lambda^{*}+U^{*}(\chi) \beta}-\frac{1}{z_{\max }}\right)^{+} \mid \mathcal{S}_{4}\right] \\
& \leq \frac{1}{\Lambda^{*}} \sum_{k=1}^{4} \operatorname{Pr}\left(\mathcal{S}_{k}\right) \quad=\frac{1}{\Lambda^{*}}
\end{aligned}
$$

Suppose that $\lim _{M \rightarrow \infty} \Lambda_{M}^{*}=0$. For $\epsilon>0$, there exists $M_{o}$ such that, for $M \geq M_{o}$, $\Lambda_{M}^{*} \leq \epsilon$. Therefore, $\left(\frac{1}{\Lambda_{M}^{*}}-\frac{1}{z_{\max }}\right)^{+} \geq\left(\frac{1}{\epsilon}-\frac{1}{z_{\max }}\right)^{+}$. For $M$ is large enough, $\frac{1}{z_{\max }}$ converges to 0 in probability, implying that, $\left(\frac{1}{\epsilon}-\frac{1}{z_{\max }}\right)^{+}$converges to $\frac{1}{\epsilon}$ in probability. Finally, it means that $\left(\frac{1}{\Lambda_{M}^{*}}-\frac{1}{z_{\max }}\right)^{+} \geq \frac{1}{\epsilon}$ with high probability. So, with an arbitrary small $\epsilon$, SU will violate the power constraint with high probability if $\lim _{M \rightarrow \infty} \Lambda_{M}^{*}=0$. Consequently, $\lim _{M \rightarrow \infty} \Lambda_{M}^{*}>0$.

Now, we are sure that $\Lambda_{M}^{*}$ will not converge to zero. Next, we will show that when $M$ is large enough, $\Lambda_{M}^{*}$ is lower-bounded by $\frac{\epsilon_{p}^{0}}{P_{a v}}$.

$$
\begin{aligned}
P_{a v} & =\sum_{k=1}^{3} \operatorname{Pr}\left(\mathcal{S}_{k}\right) E\left[\left(\frac{1}{\Lambda_{M}^{*}}-\frac{1}{z_{\max }}\right)^{+} \mid \mathcal{S}_{k}\right]+\operatorname{Pr}\left(\mathcal{S}_{4}\right) E\left[\left(\frac{1}{\Lambda_{M}^{*}+U^{*}(\chi) \beta}-\frac{1}{z_{\max }}\right)^{+} \mid \mathcal{S}_{4}\right] \\
& >\operatorname{Pr}\left(\mathcal{S}_{1}\right) E\left[\left(\frac{1}{\Lambda_{M}^{*}}-\frac{1}{z_{\max }}\right)^{+} \mid \mathcal{S}_{1}\right] \\
& =\epsilon_{p}^{0} E\left[\left(\frac{1}{\Lambda_{M}^{*}}-\frac{1}{z_{\max }}\right)^{+} \mid \mathcal{S}_{1}\right]
\end{aligned}
$$


B.8. Lower bound on $k_{o}$ when $\epsilon_{p}>\epsilon_{p}^{0}$ in ATPC with ON-OFF power control at the primary user

Since

$$
\begin{aligned}
& \lim _{M \rightarrow \infty} E\left[\left(\frac{1}{\Lambda_{M}^{*}}-\frac{1}{z_{\max }}\right)^{+} \mid \mathcal{S}_{1}\right] \\
& =\lim _{M \rightarrow \infty}\left[\frac{1}{\Lambda_{M}^{*}}\left(1-F_{Z}^{M}\left(\Lambda_{M}^{*} \mid \mathcal{S}_{1}\right)\right)-\int_{z_{\max } \Lambda_{M}^{*}}^{\infty} \frac{1}{z_{\max }} d F_{Z}^{M}\left(z_{\max } \mid \mathcal{S}_{1}\right)\right]=\frac{1}{\Lambda_{M}^{*}}
\end{aligned}
$$

It is because, as $M$ is large enough, $F_{Z}^{M}\left(\Lambda_{M}^{*} \mid \mathcal{S}_{1}\right)$ and $\int_{z_{\max }=\Lambda_{M}^{*}}^{\infty} \frac{1}{z_{\max }} d F_{Z}^{M}\left(z_{\max } \mid \mathcal{S}_{1}\right)$ approach zero. Finally, we have $\lim _{M \rightarrow \infty} \Lambda_{M}^{*}>\frac{\epsilon_{p}^{0}}{P_{a v}}$.

\section{B.8 Lower bound on $k_{o}$ when $\epsilon_{p}>\epsilon_{p}^{0}$ in ATPC with ON-OFF power control at the primary user}

Since $\operatorname{Pr}\left\{\mathcal{S}_{1}\right\}=\epsilon_{p}^{0}, \operatorname{Pr}\left\{\mathcal{S}_{3}\right\}=\epsilon_{p}-\epsilon_{p}^{0}$ and we have

$$
\begin{aligned}
\epsilon_{p}-\epsilon_{p}^{0} & =\int_{z_{\max }=\frac{\Lambda^{*}}{k_{o}}}^{\infty} \int_{\theta=0}^{\frac{k_{o}}{\Lambda^{*}}-\frac{1}{z_{\max }}} d F_{\Theta}\left(\theta \mid \mathcal{S}_{1}^{c}\right) \cdot d F_{Z}^{M}\left(z_{\max } \mid \mathcal{S}_{1}^{c}\right) \cdot \int_{g=g_{T}}^{\infty} d F_{G}(g) \\
\therefore \gamma_{\epsilon} & =\frac{\left(\epsilon_{p}-\epsilon_{p}^{0}\right)}{\int_{g=g_{T}}^{\infty} d F_{G}(g)}=\left(\epsilon_{p}-\epsilon_{p}^{0}\right) /\left(1-\epsilon_{p}^{0}\right) \\
& =\int_{z_{\max }=\frac{\Lambda^{*}}{k_{o}}}^{\infty}\left(\int_{\theta=0}^{\frac{k_{o}}{\Lambda^{*}}-\frac{1}{z_{\max }}} d F_{\Theta}\left(\theta \mid \mathcal{S}_{1}^{c}\right)\right) d F_{Z}^{M}\left(z_{\max } \mid \mathcal{S}_{1}^{c}\right) \\
& =\int_{z_{\max }=\frac{\Lambda^{*}}{k_{o}}}^{\infty}\left(1-\frac{1}{1+c_{o}\left(\frac{k_{o}}{\Lambda^{*}}-\frac{1}{z_{\max }}\right)}\right) d F_{Z}^{M}\left(z_{\max } \mid \mathcal{S}_{1}^{c}\right) \\
\leq & \left(1-\frac{1}{1+\frac{k_{o} c_{o}}{\Lambda^{*}}}\right) \cdot \int_{z_{\max }=\frac{\Lambda^{*}}{k_{o}}}^{\infty} d F_{Z}^{M}\left(z_{\max } \mid \mathcal{S}_{1}^{c}\right) \\
\leq & 1-\frac{1}{1+\frac{k_{o} c_{o}}{\Lambda^{*}}}
\end{aligned}
$$

We finally have $k_{o} \geq \frac{\Lambda^{*}}{c_{o}} \frac{\gamma_{\epsilon}}{1-\gamma_{\epsilon}}$. 
B.9. Lower bound on $E\left[r_{s}^{*} \mid \mathcal{S}_{1}^{c}\right]$ when $\epsilon_{p}=\epsilon_{p}^{0}$ in ATPC with ON-OFF power control at the primary user

\section{B.9 Lower bound on $E\left[r_{s}^{*} \mid \mathcal{S}_{1}^{c}\right]$ when $\epsilon_{p}=\epsilon_{p}^{0}$ in ATPC with ON-OFF power control at the primary user}

$$
\begin{aligned}
E\left[r_{s}^{*} \mid \mathcal{S}_{1}^{c}\right]= & \int_{z_{\max }=\Lambda^{*}}^{\infty} \int_{\theta=\frac{1}{\Lambda^{*}}-\frac{1}{z_{\max }}}^{\infty} \log \left(\frac{z_{\max }}{\Lambda^{*}}\right) d F_{\Theta}\left(\theta \mid \mathcal{S}_{1}^{c}\right) d F_{Z}^{M}\left(z_{\max } \mid \mathcal{S}_{1}^{c}\right) \\
& +\int_{z_{\max }=\Lambda^{*}}^{\infty} \int_{\theta=0}^{\infty} \log \left(1+z_{\max } \theta\right) d F_{\Theta}\left(\theta \mid \mathcal{S}_{1}^{c}\right) d F_{Z}^{M}\left(z_{\max } \mid \mathcal{S}_{1}^{c}\right) \\
= & \int_{z_{\max }=\Lambda^{*}}^{\infty} \log \left(\frac{z_{\max }}{\Lambda^{*}}\right)\left(1-F_{\Theta}\left(\theta=\frac{1}{\Lambda^{*}}-\frac{1}{z_{\max }} \mid \mathcal{S}_{1}^{c}\right)\right) d F_{Z}^{M}\left(z_{\max } \mid \mathcal{S}_{1}^{c}\right) \\
& -\int_{z_{\max }=\Lambda^{*}}^{\infty} \log \left(\frac{z_{\max }}{\Lambda^{*}}\right)\left(1-F_{\Theta}\left(\theta=\frac{1}{\Lambda^{*}}-\frac{1}{z_{\max }} \mid \mathcal{S}_{1}^{c}\right)\right) d F_{Z}^{M}\left(z_{\max } \mid \mathcal{S}_{1}^{c}\right) \\
& +\int_{z_{\max }=\Lambda^{*}}^{\infty} \frac{z_{\max }}{z_{\max }-c_{o}} \log \left(\frac{\frac{z_{\max }}{\Lambda^{*}}}{1+c_{o}\left(\frac{1}{\Lambda^{*}}-\frac{1}{z_{\max }}\right)}\right) d F_{Z}^{M}\left(z_{\max } \mid \mathcal{S}_{1}^{c}\right) \\
= & \int_{z_{\max }=\Lambda^{*}}^{\infty} \frac{z_{\max }}{z_{\max }-c_{o}} \log \left(\frac{\frac{z_{\max }}{\Lambda^{*}}}{1+c_{o}\left(\frac{1}{\Lambda^{*}}-\frac{1}{z_{\max }}\right)}\right) d F_{Z}^{M}\left(z_{\max } \mid \mathcal{S}_{1}^{c}\right) \\
= & \int_{z_{\max }=\Lambda^{*}}^{\infty} \frac{z_{\max }}{z_{\max }-c_{o}} \log \left(\frac{z_{\max }}{c_{o}}\right) d F_{Z}^{M}\left(z_{\max } \mid \mathcal{S}_{1}^{c}\right) \\
& +\int_{z_{\max }=\Lambda^{*}}^{\infty} \frac{z_{\max }}{z_{\max }-c_{o}} \log \left(\frac{c_{o}}{1+c_{o}\left(\frac{1}{\Lambda^{*}}-\frac{1}{z_{\max }}\right)}\right) d F_{Z}^{M}\left(z_{\max } \mid \mathcal{S}_{1}^{c}\right)
\end{aligned}
$$

The lower bound for $E\left[r_{s}^{*} \mid \mathcal{S}_{1}^{c}\right]$ is split in to two cases in (B.32) for $c_{o} \leq \Lambda^{*}$ and (B.33) for $c_{o} \geq \Lambda^{*}$.

For $c_{o} \leq \Lambda^{*}$,

$$
\begin{aligned}
& E\left[r_{s}^{*} \mid \mathcal{S}_{1}^{c}\right] \\
& =\int_{z_{\max }=\Lambda^{*}}^{\infty} \frac{z_{\max }}{z_{\max }-c_{o}} \log \left(\frac{z_{\max }}{c_{o}}\right) d F_{Z}^{M}\left(z_{\max } \mid g \geq g_{T}\right) \\
& +\int_{z_{\max }=\Lambda^{*}}^{\infty} \frac{z_{\max }}{z_{\max }-c_{o}} \log \left(\frac{c_{o}}{1+c_{o}\left(\frac{1}{\Lambda^{*}}-\frac{1}{z_{\max }}\right)}\right) d F_{Z}^{M}\left(z_{\max } \mid g \geq g_{T}\right) \\
& >\int_{z_{\max }=\Lambda^{*}}^{\infty} \log \left(\frac{z_{\max }}{c_{o}}\right) d F_{Z}^{M}\left(z_{\max } \mid \mathcal{S}_{1}^{c}\right)+\int_{z_{\max }=\Lambda^{*}}^{\infty} \frac{z_{\max }}{z_{\max }-c_{o}} \log \left(\frac{c_{o}}{1+c_{o}\left(\frac{1}{\Lambda^{*}}-\frac{1}{z_{\max }}\right)}\right) d F_{Z}^{M}\left(z_{\max } \mid \mathcal{S}_{1}^{c}\right) \\
& =\int_{z_{\max }=\Lambda^{*}}^{\infty} \log \left(\frac{z_{\max }}{\Lambda^{*}}\right) d F_{Z}^{M}\left(z_{\max } \mid \mathcal{S}_{1}^{c}\right)-\int_{z_{\max }=\Lambda^{*}}^{\infty} \log \left(\frac{c_{o}}{\Lambda^{*}}\right) d F_{Z}^{M}\left(z_{\max } \mid \mathcal{S}_{1}^{c}\right) \\
& +\int_{z_{\max }=\Lambda^{*}}^{\infty} \frac{z_{\max }}{z_{\max }-c_{o}} \log \left(\frac{c_{o}}{1+c_{o}\left(\frac{1}{\Lambda^{*}}-\frac{1}{z_{\max }}\right)}\right) d F_{Z}^{M}\left(z_{\max } \mid \mathcal{S}_{1}^{c}\right)
\end{aligned}
$$


B.9. Lower bound on $E\left[r_{s}^{*} \mid \mathcal{S}_{1}^{c}\right]$ when $\epsilon_{p}=\epsilon_{p}^{0}$ in ATPC with ON-OFF power control at the primary user

$$
\begin{aligned}
& \geq \int_{z_{\max }=\Lambda^{*}}^{\infty} \log \left(\frac{z_{\max }}{\Lambda^{*}}\right) d F_{Z}^{M}\left(z_{\max } \mid \mathcal{S}_{1}^{c}\right)-\log \left(\frac{c_{o}}{\Lambda^{*}}\right)\left[1-F_{Z}^{M}\left(z_{\max }=\Lambda^{*} \mid \mathcal{S}_{1}^{c}\right)\right] \\
& +\frac{1}{1-\frac{c_{o}}{\Lambda^{*}}}\left[1-F_{Z}^{M}\left(z_{\max }=\Lambda^{*} \mid \mathcal{S}_{1}^{c}\right)\right] \\
& =\int_{z_{\max }=\Lambda^{*}}^{\infty} \log \left(\frac{z_{\max }}{\Lambda^{*}}\right) d F_{Z}^{M}\left(z_{\max } \mid \mathcal{S}_{1}^{c}\right)+\left[1-F_{Z}^{M}\left(z_{\max }=\Lambda^{*} \mid \mathcal{S}_{1}^{c}\right)\right] \frac{\frac{c_{o}}{\Lambda^{*}}}{1-\frac{c_{o}}{\Lambda^{*}}} \log \frac{c_{o}}{\Lambda^{*}} \\
& >\int_{z_{\max }=\Lambda^{*}}^{\infty} \log \left(\frac{z_{\max }}{\Lambda^{*}}\right) d F_{Z}^{M}\left(z_{\max } \mid \mathcal{S}_{1}^{c}\right)+\frac{c_{o}}{1-\frac{c_{o}}{\Lambda^{*}}} \log \frac{c_{o}}{\Lambda^{*}} \\
& =E\left[\log \left(\frac{z_{\max }}{\Lambda^{*}}\right) 1_{\left\{z_{\max } \geq \Lambda^{*}\right\}} \mid \mathcal{S}_{1}^{c}\right]+\frac{\frac{c_{o}}{\Lambda^{*}}}{1-\frac{c_{o}}{\Lambda^{*}}} \log \frac{c_{o}}{\Lambda^{*}}
\end{aligned}
$$

For (B.32), the first inequality is from $\frac{z_{\max }}{z_{\max }-c_{o}} \log \left(\frac{z_{\max }}{c_{o}}\right)>\log \left(\frac{z_{\max }}{c_{o}}\right)$. The second inequality is from the lower bound of $Q\left(z_{\max }\right)=\frac{z_{\max }}{z_{\max }-c_{o}} \log \left(\frac{c_{o}}{1+c_{o}\left(\frac{1}{\Lambda^{*}}-\frac{1}{z_{\max }}\right)}\right)$ which is $\frac{1}{1-\frac{c_{o}}{\Lambda^{*}}} \log \frac{c_{o}}{\Lambda^{*}}$. Note that $Q\left(z_{\max }\right)$ is increasing function and always negative but bounded over the range $\Lambda^{*} \leq z_{\max } \leq \infty$. Thus, $\frac{1}{1-\frac{c_{o}}{\Lambda^{*}}} \log \frac{c_{o}}{\Lambda^{*}} \leq Q\left(z_{\max }\right) \leq$ $\log \left(\frac{\frac{c_{O}}{\Lambda^{*}}}{1+\frac{c_{O}}{\Lambda^{*}}}\right)<0$. The last inequality is from $\frac{\frac{c_{O}}{\Lambda^{*}}}{1-\frac{c_{O}}{\Lambda^{*}}} \log \frac{c_{o}}{\Lambda^{*}} \leq 0$ and increasing in $z_{\max }$, while $\left[1-F_{Z}^{M}\left(z_{\max }=\Lambda^{*} \mid g \geq g_{T}\right)\right]<1$. Further, as $c_{o} \leq \Lambda^{*} \leq \frac{1}{P_{a v}}$, the minimum of $\frac{\frac{c_{o}}{\Lambda^{*}}}{1-\frac{c_{o}}{\Lambda^{*}}} \log \frac{c_{o}}{\Lambda^{*}}$ is $-\frac{1}{c_{o}}$.

For $c_{o} \geq \Lambda^{*}$,

$$
\begin{aligned}
& E\left[r_{s}^{*} \mid \mathcal{S}_{1}^{c}\right] \\
& =\int_{z_{\max }=\Lambda^{*}}^{\infty} \frac{z_{\max }}{z_{\max }-c_{o}} \log \left(\frac{z_{\max }}{c_{o}}\right) d F_{Z}^{M}\left(z_{\max } \mid \mathcal{S}_{1}^{c}\right) \\
& +\int_{z_{\max }=\Lambda^{*}}^{\infty} \frac{z_{\max }}{z_{\max }-c_{o}} \log \left(\frac{c_{o}}{1+c_{o}\left(\frac{1}{\Lambda^{*}}-\frac{1}{z_{\max }}\right)}\right) d F_{Z}^{M}\left(z_{\max } \mid \mathcal{S}_{1}^{c}\right) \\
& =\int_{z_{\max }=c_{o}}^{\infty} \frac{z_{\max }-c_{o}}{z_{\max }} \log \left(\frac{z_{\max }}{c_{o}}\right) d F_{Z}^{M}\left(z_{\max } \mid \mathcal{S}_{1}^{c}\right)+\int_{z_{\max }=\Lambda^{*}}^{c_{o}} \frac{z_{\max }-c_{o}}{z_{\max }} \log \left(\frac{z_{\max }}{c_{o}}\right) d F_{Z}^{M}\left(z_{\max } \mid \mathcal{S}_{1}^{c}\right) \\
& +\int_{z_{\max }=\Lambda^{*}}^{\infty} \frac{z_{\max }}{z_{\max }-c_{o}} \log \left(\frac{c_{o}}{1+c_{o}\left(\frac{1}{\Lambda^{*}}-\frac{1}{z_{\max }}\right)}\right) d F_{Z}^{M}\left(z_{\max } \mid \mathcal{S}_{1}^{c}\right) \\
& >\int_{z_{\max }=c_{o}}^{\infty} \log \left(\frac{z_{\max }}{c_{o}}\right) d F_{Z}^{M}\left(z_{\max } \mid \mathcal{S}_{1}^{c}\right)+\int_{\max }^{c_{o}}=\Lambda^{*} \frac{z_{\max }}{z_{\max }-c_{o}} \log \left(\frac{z_{\max }}{c_{o}}\right) d F_{Z}^{M}\left(z_{\max } \mid \mathcal{S}_{1}^{c}\right) \\
& +\int_{z_{\max }=\Lambda^{*}}^{\infty} \frac{z_{\max }}{z_{\max }-c_{o}} \log \left(\frac{c_{o}}{1+c_{o}\left(\frac{1}{\Lambda^{*}}-\frac{1}{z_{\max }}\right)}\right) d F_{Z}^{M}\left(z_{\max } \mid \mathcal{S}_{1}^{c}\right) \\
& \geq \int_{z_{\max }=c_{o}}^{\infty} \log \left(\frac{z_{\max }}{c_{o}}\right) d F_{Z}^{M}(.)+\frac{\log \left(\frac{\Lambda^{*}}{c_{o}}\right)}{1-\frac{c_{o}}{\Lambda^{*}}} \int_{z_{\max }=\Lambda^{*}}^{c_{o}} d F_{Z}^{M}(.)+\frac{1}{1-\frac{c_{o}}{\Lambda^{*}}} \log \frac{c_{o}}{\Lambda^{*}} \int_{z_{\max }=\Lambda^{*}} d F_{Z}^{M}\left(z_{\max } \mid \mathcal{S}_{1}^{c}\right) \\
& =\int_{z_{\max }=c_{o}}^{\infty} \log \left(\frac{z_{\max }}{c_{o}}\right) d F_{Z}^{M}(.)+\left[1-F_{Z}^{M}\left(c_{o} \mid \mathcal{S}_{1}^{c}\right)\right] \frac{\log \left(\frac{c_{o}}{\Lambda^{*}}\right)}{1-\frac{c_{o}}{\Lambda^{*}}} \\
& >\int_{z_{\max }=c_{o}}^{\infty} \log \left(\frac{z_{\max }}{c_{o}}\right) d F_{Z}^{M}(.)+\frac{\log \left(\frac{c_{o}}{\Lambda^{*}}\right)}{1-\frac{c_{o}}{\Lambda^{*}}} \\
& =E\left[\log \left(\frac{z_{\max }}{c_{o}}\right) 1_{\left\{z_{\max } \geq c_{o}\right\}} \mid \mathcal{S}_{1}^{c}\right]+\frac{\log \left(\frac{c_{o}}{\Lambda^{*}}\right)}{1-\frac{c_{o}}{\Lambda^{*}}}
\end{aligned}
$$


For (B.33), the first inequality is from $\frac{z_{\max }}{z_{\max }-c_{o}} \log \left(\frac{z_{\max }}{c_{o}}\right)>\log \left(\frac{z_{\max }}{c_{o}}\right)$. The second inequality is due to the minimum of $\frac{z_{\max }}{z_{\max }-c_{o}} \log \left(\frac{z_{\max }}{c_{o}}\right)$ over $\Lambda^{*} \leq z \leq c_{o}$ is $\frac{\Lambda^{*}}{\Lambda^{*}-c_{o}} \log \left(\frac{\Lambda^{*}}{c_{o}}\right)$ and the lower bound of $Q\left(z_{\max }\right)$ which is $\frac{1}{1-\frac{c_{O}}{\Lambda^{*}}} \log \frac{c_{o}}{\Lambda^{*}}$. The last inequality is because of $0<\left[1-F_{Z}^{M}\left(c_{o} \mid g \geq g_{T}\right)\right]<1$, while $\frac{\log \left(\frac{c_{o}}{\Lambda^{*}}\right)}{1-\frac{c_{o}}{\Lambda^{*}}} \leq 0$. Also note that, as $0<\Lambda^{*} \leq c_{o}$, $\frac{\log \left(\frac{c_{o}}{\Lambda^{*}}\right)}{1-\frac{c_{o}}{\Lambda^{*}}}$ will not go to $-\infty$.

\section{B.10 Conclusion for throughput scaling in ATPC case}

As $0<\Lambda^{*} \leq \frac{1}{P_{a v}}$, the behaviour of throughput in secondary network can be shown as follows.

- Upper-bound : From (3.16), we can analyze the upper-bound

$$
\lim _{M \rightarrow \infty} \frac{C_{s}^{*}}{\log (\log M)} \leq \lim _{M \rightarrow \infty} \frac{E\left[\log \left(\frac{z_{\max }}{\Lambda^{*}}\right) 1_{\left\{z_{\max } \geq \Lambda^{*}\right\}}\right]}{\log (\log M)} \stackrel{(c)}{=} 1
$$

(c) is because we can also show the convergence in mean for both the set $\mathcal{S}_{1}$ and $\mathcal{S}_{1}^{c}$ by the same technique in [81].

- Lower-bound :

$$
\begin{aligned}
& - \text { If } \epsilon_{p}>\epsilon_{p}^{0} \\
& \quad \lim _{M \rightarrow \infty} \frac{C_{s}^{*}}{\log (\log M)} \\
& \geq \lim _{M \rightarrow \infty} \frac{1}{\log (\log M)}\left(E\left[\log \left(\frac{z_{\max }}{\Lambda^{*}}\right) 1_{\left\{z_{\max } \geq \Lambda^{*}\right\}}\right]+\log \left(\frac{\Lambda^{*}}{c_{o}} \cdot \frac{\gamma_{\epsilon}}{1-\gamma_{\epsilon}}\right)\right) \\
& =\lim _{M \rightarrow \infty} \frac{1}{\log (\log M)} E\left[\log \left(\frac{z_{\max }}{\Lambda^{*}}\right) 1_{\left\{z_{\max } \geq \Lambda^{*}\right\}}\right]+\lim _{M \rightarrow \infty} \frac{1}{\log (\log M)} \log \left(\frac{\Lambda^{*}}{c_{o}} \cdot \frac{\gamma_{\epsilon}}{1-\gamma_{\epsilon}}\right) \\
& =1 \\
& - \text { If } \epsilon_{p}=\epsilon_{p}^{0}
\end{aligned}
$$




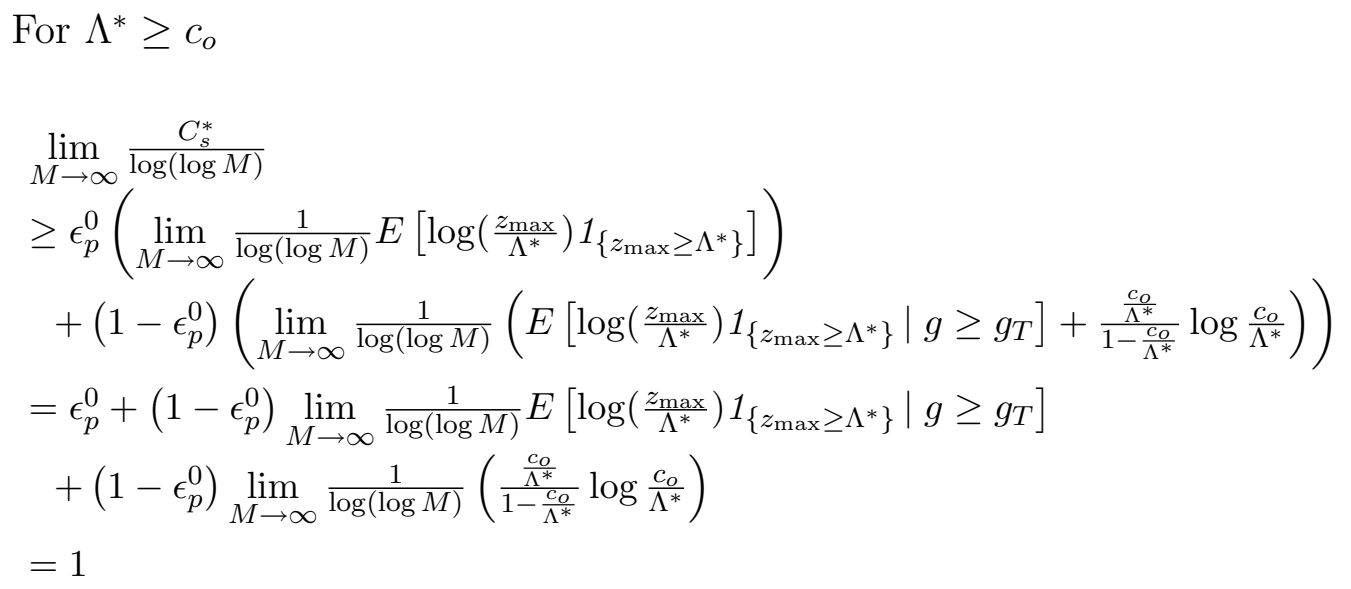

For $\Lambda^{*} \leq c_{o}$

$$
\begin{aligned}
& \lim _{M \rightarrow \infty} \frac{C_{s}^{*}}{\log (\log M)} \\
& \geq \epsilon_{p}^{0}\left(\lim _{M \rightarrow \infty} \frac{1}{\log (\log M)} E\left[\log \left(\frac{z_{\max }}{\Lambda^{*}}\right) 1_{\left\{z_{\max } \geq \Lambda^{*}\right\}}\right]\right) \\
& \quad+\left(1-\epsilon_{p}^{0}\right)\left(\lim _{M \rightarrow \infty} \frac{1}{\log (\log M)}\left(E\left[\log \left(\frac{z_{\max }}{c_{o}}\right) 1_{\left\{z_{\max } \geq c_{o}\right\}} \mid g \geq g_{T}\right]+\frac{1}{1-\frac{c_{o}}{\Lambda^{*}}} \log \frac{c_{o}}{\Lambda^{*}}\right)\right) \\
& =\epsilon_{p}^{0}+\left(1-\epsilon_{p}^{0}\right) \lim _{M \rightarrow \infty} \frac{1}{\log (\log M)} E\left[\log \left(\frac{z_{\max }}{c_{o}}\right) 1_{\left\{z_{\max } \geq c_{o}\right\}} \mid g \geq g_{T}\right] \\
& \quad+\left(1-\epsilon_{p}^{0}\right) \lim _{M \rightarrow \infty} \frac{1}{\log (\log M)}\left(\frac{1}{1-\frac{c_{O}}{\Lambda^{*}}} \log \frac{c_{o}}{\Lambda^{*}}\right) \\
& =1
\end{aligned}
$$

Finally, we can conclude that $C_{s}^{*}$ grows like $\log (\log M)$ in ATPC case. Finally, we can conclude that $C_{s}^{*}$ grows like $\log (\log M)$ in ATPC case.

\section{B.11 Proof of Lemma 3.3.3}

Proof. When SBS transmits by power $P_{o}$, KKT condition $\left(\frac{1}{1+\sum_{i=1}^{M} p_{2 i}^{*}(\chi) z_{i}} z_{i}-\Lambda^{*}(\chi)\right)$ must equal to zero, thereby $\Lambda^{*}(\chi)=\frac{z_{\max }}{1+P_{o} z_{\max }}$. Also, the boundary between the region 3 and 4 has to satisfy the condition $\mathscr{B}_{1, \chi}^{B}=\mathscr{B}_{2, \chi}^{B}$. Therefore,

$$
\begin{aligned}
& 0=\mathscr{B}_{1, \chi}^{B}-\mathscr{B}_{2, \chi}^{B}=\left[S^{*}+\log \left(1+\theta z_{\max }\right)\right]-\left[\log \left(1+P_{o} z_{\max }\right)-\Lambda^{*}(\chi) P_{o}\right] \\
& \therefore \quad \frac{1+\theta z_{\max }}{1+P_{o} z_{\max }}=\exp \left(-S^{*}-\frac{P_{o} z_{\max }}{1+P_{o} z_{\max }}\right) \\
& \therefore \quad \theta=K\left(z_{\max }\right)=-\frac{1}{z_{\max }}+\frac{1}{z_{\max }}\left(1+P_{o} z_{\max }\right) \exp \left(-S^{*}-\frac{P_{o} z_{\max }}{1+P_{o} z_{\max }}\right)
\end{aligned}
$$


Next, we will show that $K\left(z_{\max }\right)$ is strictly increasing over $z_{\max } \geq 0$ when POC is active, i.e. $S^{*}>0$.

$$
\begin{array}{ll} 
& K^{\prime}(z)=\frac{1}{z^{2}}\left[1-\left(\frac{1+2 P_{o} z}{1+P_{o} z}\right) \exp \left(-S^{*}-\frac{P_{o} z}{1+P_{o} z}\right)\right] \\
\text { Let } & f(z)=\left(\frac{1+2 P_{o} z}{1+P_{o} z}\right) \exp \left(-S^{*}-\frac{P_{o} z}{1+P_{o} z}\right) \geq 0 . \text { Thus, } \\
& f^{\prime}(z)=-\exp \left(-S^{*}-\frac{P_{o} z}{1+P_{o} z}\right) \frac{P_{o}^{2}}{\left(1+P_{o} z\right)^{3}} \leq 0, \text { for } z \geq 0 \\
& \therefore f(z) \text { is decreasing for } 0 \leq z \leq \infty, \text { so } \\
& f(z) \leq f(z=0) \quad \leq \exp \left(-S^{*}\right) \leq 1 \\
& \therefore K^{\prime}(z)=\frac{1}{z^{2}}(1-f(z)) \geq \frac{1}{z^{2}}\left(1-\exp \left(-S^{*}\right)\right)>0 \text { for } S^{*}>0
\end{array}
$$

\section{B.12 Expression of $\delta\left(P_{o}, c_{o}, \gamma_{\epsilon}\right)$}

First, we will show that $S^{*}$ has an upper bound in this case because we know that $\operatorname{Pr}\left\{\mathcal{S}_{3}\right\}=\epsilon_{p}-\epsilon_{p}^{0}$. Define $\gamma_{\epsilon}=\left(\epsilon_{p}-\epsilon_{p}^{0}\right) /\left(1-\epsilon_{p}^{0}\right)$. Then, we have

$$
\begin{aligned}
\gamma_{\epsilon} & =\int_{z_{\max }=z_{o}}^{\infty}\left(\int_{\theta=0}^{K\left(z_{\max }\right)} d F_{\Theta}\left(\theta \mid g \geq g_{T}\right)\right) d F_{Z}^{M}\left(z_{\max } \mid g \geq g_{T}\right) \\
& =\int_{z_{\max }=z_{o}}^{\infty}\left(1-\frac{1}{1+c_{o} K\left(z_{\max }\right)}\right) d F_{Z}^{M}\left(z_{\max } \mid g \geq g_{T}\right) \\
& \leq\left(1-\frac{1}{1+c_{o} K(\infty)}\right)
\end{aligned}
$$

Note that The inequality is from the fact that $\left(1-\frac{1}{1+c_{o} K\left(z_{\max }\right)}\right) \leq\left(1-\frac{1}{1+c_{o} K(\infty)}\right)$ and $K(\infty)=P_{o} \exp \left(-S^{*}-1\right)$, the condition on $S^{*}$ in this case becomes $0<S^{*} \leq$ $\log \left(P_{o} c_{o} \frac{1-\gamma_{\epsilon}}{\gamma_{\epsilon}}\right)-1$.

According to the condition on the set 4 that $\mathscr{B}_{1, \chi}^{B} \geq \mathscr{B}_{2, \chi}^{B}$. We have

$$
\begin{aligned}
& S^{*}+\log \left(1+\theta z_{\max }\right) \geq \log \left(1+P_{o} z_{\max }\right)-\left[1-\frac{1}{1+P_{o} z_{\max }}\right] \\
& \therefore \log \left(1+\theta z_{\max }\right) \geq \log \left(1+P_{o} z_{\max }\right)-S^{*}-1+\frac{1}{1+P_{o} z_{\max }} \geq \log \left(1+P_{o} z_{\max }\right)-S^{*}-1 \\
& \therefore \log \left(1+\theta z_{\max }\right) \geq \log \left(1+P_{o} z_{\max }\right)-\left[\log \left(P_{o} c_{o} \frac{1-\gamma_{\epsilon}}{\gamma_{\epsilon}}\right)-1\right]-1=\log \left(1+P_{o} z_{\max }\right)-\log \left(P_{o} c_{o} \frac{1-\gamma_{\epsilon}}{\gamma_{\epsilon}}\right) \\
& \therefore E\left[\log \left(1+\theta z_{\max }\right) \mid \mathcal{S}_{4}\right] \geq E\left[\log \left(1+P_{o} z_{\max }\right) \mid \mathcal{S}_{4}\right]-\delta\left(P_{o}, c_{o}, \gamma_{\epsilon}\right) \\
& \therefore C_{s}^{*} \geq \sum_{k=1}^{4} \operatorname{Pr}\left(\mathcal{S}_{k}\right) E\left[\log \left(1+P_{o} z_{\max }\right) \mid \mathcal{S}_{k}\right]-\operatorname{Pr}\left\{\mathcal{S}_{4}\right\} \delta\left(P_{o}, c_{o}, \gamma_{\epsilon}\right) \\
& \quad \geq E\left[\log \left(1+P_{o} z_{\max }\right)\right]-\delta\left(P_{o}, c_{o}, \gamma_{\epsilon}\right)
\end{aligned}
$$


B.13. Lower bound on $E\left[r_{s}^{*} \mid g \geq g_{T}\right]$ when $\epsilon_{p}=\epsilon_{p}^{0}$ for PTPC with ON-OFF power policy at the $P U$

\section{B.13 Lower bound on $E\left[r_{s}^{*} \mid g \geq g_{T}\right]$ when $\epsilon_{p}=\epsilon_{p}^{0}$ for PTPC with ON-OFF power policy at the PU}

$$
\begin{aligned}
E & {\left[r_{s}^{*} \mid g \geq g_{T}\right] } \\
= & {\left[\int_{z_{\max }=0}^{\infty} \int_{\theta=P_{o}}^{\infty} \log \left(1+z_{\max } P_{o}\right) d F_{\Theta}\left(\theta \mid g \geq g_{T}\right) d F_{Z}^{M}\left(z_{\max } \mid g \geq g_{T}\right)\right] } \\
& +\left(\int_{z_{\max }=0}^{\infty}\left(\int_{\theta=0}^{P_{o}} \log \left(1+z_{\max } \theta\right) d F_{\Theta}\left(\theta \mid g \geq g_{T}\right)\right) d F_{Z}^{M}\left(z_{\max } \mid g \geq g_{T}\right)\right) \\
= & {\left[\int_{z_{\max }=0}^{\infty} \log \left(1+z_{\max } P_{o}\right)\left(1-F_{\Theta}\left(\theta=P_{o} \mid g \geq g_{T}\right)\right) d F_{Z}^{M}\left(z_{\max } \mid g \geq g_{T}\right)\right] } \\
& +\left(\int_{z_{\max }=0}^{\infty} \frac{z_{\max }}{z_{\max }-c_{o}} \log \left(\frac{1+P_{o} z_{\max }}{1+P_{o} c_{o}}\right) d F_{Z}^{M}\left(z_{\max } \mid g \geq g_{T}\right)\right. \\
= & \int_{z_{\max }=0}^{\infty} \int_{\max }^{\infty} \frac{z_{\max }}{z_{\max }-c_{o}} \log \left(\frac{1+P_{o} z_{\max }}{1+P_{o} c_{o}}\right) d F_{Z}^{M}\left(z_{\max } \mid g \geq g_{T}\right) \\
= & \int_{z_{\max }=0}^{\infty} \frac{z_{\max }}{z_{\max }-c_{o}} \log \left(\frac{z_{\max }}{c_{o}}\right) d F_{Z}^{M}\left(z_{\max } \mid g \geq g_{T}\right) \\
& +\int_{\int_{\max }=0}^{\infty} \frac{z_{\max }}{z_{\max }-c_{o}} \log \left(\frac{P_{o}+\frac{1}{z_{\max }}}{P_{o}+\frac{1}{c_{o}}}\right) d F_{Z}^{M}\left(z_{\max } \mid g \geq g_{T}\right) \\
\geq & {\left[\int_{z_{\max }=0}^{\infty} \frac{z_{\max }}{z_{\max }-c_{o}} \log \left(\frac{z_{\max }}{c_{o}}\right) d F_{Z}^{M}\left(z_{\max } \mid g \geq g_{T}\right)\right]-\log \left(\frac{P_{o}+\frac{1}{c_{o}}}{P_{o}}\right) } \\
\geq & {\left[\int_{z_{\max }=c_{o}}^{\infty} \log \left(\frac{z_{\max }}{c_{o}}\right) d F_{Z}^{M}\left(z_{\max } \mid g \geq g_{T}\right)\right]-\log \left(\frac{P_{o}+\frac{1}{c_{o}}}{P_{o}}\right) } \\
= & E\left[\log \left(\frac{z_{\max }}{c_{o}}\right) 1\left(z_{\max } \geq c_{T}\right) \mid g \geq g_{T}\right]-\log \left(\frac{P_{o}+\frac{1}{c_{o}}}{P_{o}}\right)
\end{aligned}
$$

Remark B.13.1. The second equality in (B.35) is obtained by using integration by parts. The first inequality follows from the fact that $-\log \left(\frac{P_{o}+\frac{1}{c_{o}}}{P_{o}}\right) \leq \frac{z_{\max }}{z_{\max }-c_{o}} \log \left(\frac{P_{o}+\frac{1}{z_{\max }}}{P_{o}+\frac{1}{c_{o}}}\right) \leq$ 0 . The last inequality is from $\frac{z_{\max }}{z_{\max }-c_{o}} \log \left(\frac{z_{\max }}{c_{o}}\right) \geq 0$ for all $z_{\max } \geq 0$, using $c_{o}$ instead of 0 as the lower limit of integration and applying the fact that $\frac{z_{\max }}{z_{\max }-c_{o}} \log \left(\frac{z_{\max }}{c_{o}}\right) \geq$ $\log \left(\frac{z_{\max }}{c_{o}}\right)$. 


\section{B.14 Conclusion for throughput scaling in PTPC case}

\section{- Upper-bound :}

From (3.20), we have

$$
\begin{aligned}
& C_{s}^{*} \leq E\left[\log \left(1+P_{o} z_{\max }\right)\right] \stackrel{(a)}{\leq} \log \left(E\left[\left(z_{\max }\right)\right]\right)+\log \left(P_{o}+E\left[\frac{1}{z_{\max }}\right]\right) \\
& \therefore \lim _{M \rightarrow \infty} \frac{C_{s}^{*}}{\log (\log M)} \leq \lim _{M \rightarrow \infty} \frac{1}{\log (\log M)}\left(\log \left(E\left[z_{\max }\right]\right)+\log \left(P_{o}+E\left[\frac{1}{z_{\max }}\right]\right)\right)=1
\end{aligned}
$$

Note that the inequality (a) from (B.36) follows from Jensen's inequality.

\section{- Lower-bound :}

- When $\epsilon_{p}>\epsilon_{p}^{0}$

$$
\begin{aligned}
\lim _{M \rightarrow \infty} \frac{C_{s}^{*}}{\log (\log M)} & \geq \lim _{M \rightarrow \infty} \frac{1}{\log (\log M)} E\left[\log \left(1+P_{o} z_{\max }\right)\right]-\lim _{M \rightarrow \infty} \frac{1}{\log (\log M)} \delta\left(P_{o}, c_{o}, \gamma_{\epsilon}\right) \\
& =\lim _{M \rightarrow \infty} \frac{1}{\log (\log M)} E\left[\log \left(z_{\max }\right) 1\left(z \geq P_{o}\right)\right]=1
\end{aligned}
$$

- When $\epsilon_{p}=\epsilon_{p}^{0}$

$$
\begin{aligned}
\lim _{M \rightarrow \infty} \frac{C_{s}^{*}}{\log (\log M)} \\
=\epsilon_{p}^{0} \lim _{M \rightarrow \infty} \frac{E\left[r_{s}^{*} \mid g<g_{T}\right]}{\log (\log M)}+\left(1-\epsilon_{p}^{0}\right) \lim _{M \rightarrow \infty} \frac{E\left[r_{s}^{*} \mid g \geq g_{T}\right]}{\log (\log M)} \\
=\epsilon_{p}^{0} \lim _{M \rightarrow \infty} \frac{E\left[\log \left(1+P_{o} z_{\max }\right) \mid g<g_{T}\right]}{\log (\log M)}+\left(1-\epsilon_{p}^{0}\right) \lim _{M \rightarrow \infty} \frac{E\left[r_{s} \mid g g_{T}\right]}{\log (\log M)} \\
\geq \epsilon_{p}^{0} \lim _{M \rightarrow \infty} \frac{1}{\log (\log M)} E\left[\log \left(P_{o} z_{\max }\right) \mid g<g_{T}\right] \\
\quad+\left(1-\epsilon_{p}^{0}\right) \lim _{M \rightarrow \infty} \frac{1}{\log (\log M)}\left(E\left[\log \left(\frac{z_{\max }}{c_{o}} 1\left(z \geq c_{o}\right)\right) \mid g \geq g_{T}\right]-\log \left(\frac{P_{o}+\frac{1}{c_{o}}}{P_{o}}\right)\right) \\
=\epsilon_{p}^{0} \lim _{M \rightarrow \infty} \frac{1}{\log (\log M)}\left[E\left[\log \left(z_{\max }\right) \mid g<g_{T}\right]+\log P_{o}\right] \\
\quad+\left(1-\epsilon_{p}^{0}\right) \lim _{M \rightarrow \infty} \frac{1}{\log (\log M)} E\left[\log \left(\frac{z_{\max }}{c_{o}} 1\left(z \geq c_{o}\right)\right) \mid g \geq g_{T}\right] \\
=1
\end{aligned}
$$

Remark B.14.1. The inequality is from the fact that $E\left[\log \left(1+P_{o} z_{\max }\right) \mid g<g_{T}\right] \geq$ $E\left[\log \left(P_{o} z_{\max }\right) \mid g<g_{T}\right]$ and the result from (B.35) that $E\left[r_{s}^{*} \mid g \geq g_{T}\right] \geq$ $E\left[\log \left(\frac{z_{\max }}{c_{o}}\right) 1\left(z_{\max } \geq c_{o}\right) \mid g \geq g_{T}\right]-\log \left(\frac{P_{o}+\frac{1}{c_{o}}}{P_{o}}\right)$. 
Finally, we can conclude that $C_{s}^{*}$ grows like $\log (\log M)$ in PTPC case.

\section{B.15 Proof of Theorem 3.4.1 for $\epsilon_{p}=\epsilon_{p}^{0}$}

In this case, it implies that $\operatorname{Pr}\left(\tilde{\mathcal{S}}_{2}\right)=\epsilon_{p}-\epsilon_{p}^{0}=0$ and $\rho_{\max }=h_{\max }$ in $\tilde{\mathcal{S}}_{1}$. Then, the throughput from the SBS is expressed as $C_{s}^{*}=E\left[\log \left(\frac{\rho_{\max }}{\Lambda^{*}}\right) 1_{\left\{\rho_{\max } \geq \Lambda^{*}, \tilde{\mathcal{S}}_{1}\right\}}\right]=$ $\epsilon_{p}^{0} E\left[\log \left(\frac{h_{\max }}{\Lambda^{*}}\right)\right]$. Thus, we can conclude that $\lim _{M \rightarrow \infty} \frac{C_{s}^{*}}{\log (\log M)}=\epsilon_{p}$.

\section{B.16 Proof of Theorem 3.4.1 for $\epsilon_{p}>\epsilon_{p}^{0}$}

In this case, throughput $C_{s}^{*}$ can be described as

$$
C_{s}^{*}=E\left[\log \frac{\rho_{\max }}{\Lambda^{*}} 1_{\left\{g<g_{T}, \rho_{\max } \geq \Lambda^{*}\right\}}\right]+E\left[\log \frac{\rho_{\max }}{\Lambda^{*}} 1_{\left\{g \geq g_{T}, \rho_{\max } \geq \frac{\Lambda^{*}}{t^{-1}\left(S^{*}\right)}\right\}}\right]
$$

Note that $E\left[\log \frac{\rho_{\max }}{\Lambda^{*}} 1_{\left\{g<g_{T}, \rho_{\max } \geq \Lambda^{*}\right\}}\right]$ have been shown previously that it scales like $\epsilon_{p}^{0} \log (\log M)$. So, we have to show that $E\left[\log \frac{\rho_{\max }}{\Lambda^{*}} 1_{\left\{g \geq g_{T}, \rho_{\max } \geq \frac{\Lambda^{*}}{t^{-1}\left(S^{*}\right)}\right\}}\right]$ scales like $\left(\epsilon_{p}-\epsilon_{p}^{0}\right) \log (\log M)$. follows.

Then, we can compute the upper-bound of $E\left[\log \frac{\rho_{\max }}{\Lambda^{*}} 1_{\left\{g \geq g_{T}, \rho_{\max } \geq \frac{\Lambda^{*}}{t^{-1}\left(S^{*}\right)}\right\}}\right]$ as

$$
\begin{aligned}
& E\left[\log \frac{\rho_{\max }}{\Lambda^{*}} 1_{\left\{g \geq g_{T}, \rho_{\max } \geq \frac{\Lambda^{*}}{t^{-1}\left(S^{*}\right)}\right\}}\right] \\
& =E\left[\log \frac{\rho_{\max }}{\Lambda^{*}} 1_{\left\{\rho_{\max } \leq \log M, g \geq g_{T}, \rho_{\max } \geq \frac{\Lambda^{*}}{t^{-1}\left(S^{*}\right)}\right\}}\right] \\
& +\sum_{i=1}^{\infty} E\left[\log \frac{\rho_{\max }}{\Lambda^{*}} 1_{\left.\left\{i \log M \leq \rho_{\max } \leq(i+1) \log M, g \geq g_{T}, \rho_{\max } \geq \frac{\Lambda^{*}}{t^{-1}\left(S^{*}\right)}\right\}\right]}\right] \\
& \leq \log \left(\frac{\log M}{\Lambda^{*}}\right) \operatorname{Pr}\left(\rho_{\max } \leq \log M, g \geq g_{T}, \rho_{\max } \geq \frac{\Lambda^{*}}{t^{-1}\left(S^{*}\right)}\right) \\
& +\sum_{i=1}^{\infty} \log \left(\frac{(i+1) \log M}{\Lambda^{*}}\right) \operatorname{Pr}\left(\rho_{\max } \geq i \log M, g \geq g_{T}, \rho_{\max } \geq \frac{\Lambda^{*}}{t^{-1}\left(S^{*}\right)}\right) \\
& \stackrel{(a)}{\leq} \log \left(\frac{\log M}{\Lambda^{*}}\right) \operatorname{Pr}\left(g \geq g_{T}, \rho_{\max } \geq \frac{\Lambda^{*}}{t^{-1}\left(S^{*}\right)}\right) \\
& +\sum_{i=1}^{\infty} \log \left(\frac{(i+1) \log M}{\Lambda^{*}}\right) \operatorname{Pr}\left(\rho_{\max } \geq i \log M\right) \\
& \stackrel{(b)}{\leq}\left(\epsilon_{p}-\epsilon_{p}^{0}\right) \log \left(\frac{\log M}{\Lambda^{*}}\right)+\sum_{i=1}^{\infty} \log \left(\frac{(i+1) \log M}{\Lambda^{*}}\right) O\left(\frac{e^{O(i)}}{M^{i} O(i)}\right) \\
& \therefore \lim _{M \rightarrow \infty} \frac{E\left[\log \frac{\rho_{\max }}{\Lambda^{*}} 1\left\{g \geq g_{T}, \rho_{\max } \geq \frac{\Lambda^{*}}{t^{-1}\left(S^{*}\right)}\right\}\right]}{\log (\log M)} \stackrel{(c)}{\leq}\left(\epsilon_{p}-\epsilon_{p}^{0}\right)
\end{aligned}
$$


Remark B.16.1. The inequality from $(a)$ in is from the fact that

$$
\begin{aligned}
& \operatorname{Pr}\left(\rho \leq \log M, g \geq g_{T}, \rho_{\max } \geq \frac{\Lambda^{*}}{t^{-1}\left(S^{*}\right)}\right) \leq \operatorname{Pr}\left(g \geq g_{T}, \rho_{\max } \geq \frac{\Lambda^{*}}{t^{-1}\left(S^{*}\right)}\right) \text { and } \\
& \operatorname{Pr}\left(\rho_{\max } \geq i \log M, g \geq g_{T}, \rho_{\max } \geq \frac{\Lambda^{*}}{t^{-1}\left(S^{*}\right)}\right) \leq \operatorname{Pr}\left(\rho_{\max } \geq i \log M\right) . \text { For }(b), \text { we }
\end{aligned}
$$
use the result in [81], i.e. $\operatorname{Pr}\left(\rho_{\max } \geq i \log M\right) \leq O\left(\frac{e^{O(i)}}{M^{i} O(i)}\right)$ because $\rho_{\max }$ grows like $\log M$. In [81], it shows that $\sum_{i=1}^{\infty} \log \left(\frac{(i+1) \log M}{\Lambda^{*}}\right) O\left(\frac{e^{O(i)}}{M^{i} O(i)}\right)$ is finite, leading to the result in $(c)$.

Thus, we have

$$
\begin{aligned}
\lim _{M \rightarrow \infty} \frac{C_{s}^{*}}{\log (\log M)} & =\lim _{M \rightarrow \infty} \frac{E\left[\log \frac{\rho_{\max }}{\Lambda^{*}} 1_{\left\{g \geq g_{T}\right\}} 1_{\left\{\rho_{\max } \geq \Lambda^{*}\right\}}\right]}{\log (\log M)}+\lim _{M \rightarrow \infty} \frac{E\left[\log \frac{\rho_{\max }}{\Lambda^{*}} 1_{\left.\left\{g \geq g_{T}\right\}^{1}\left\{\rho_{\max } \geq \frac{\Lambda^{*}}{t^{-1}\left(S^{*}\right)}\right\}\right]}\right.}{\log (\log M)} \\
& \leq \epsilon_{p}^{0}+\left(\epsilon_{p}-\epsilon_{p}^{0}\right)=\epsilon_{p}
\end{aligned}
$$

The lower bound of $E\left[\log \frac{\rho_{\max }}{\Lambda^{*}} 1_{\left\{g \geq g_{T}, \rho_{\max } \geq \frac{\Lambda^{*}}{t^{-1}\left(S^{*}\right)}\right\}}\right]$ can be obtained as follows

$$
\begin{aligned}
E\left[\log \frac{\rho_{\max }}{\Lambda^{*}} 1_{\left.\left\{g \geq g_{T}, \rho_{\max } \geq \frac{\Lambda^{*}}{t^{-1}\left(S^{*}\right)}\right\}\right]}\right. & \geq E\left[\log \left(\frac{1}{t^{-1}\left(S^{*}\right)}\right) 1_{\left\{g \geq g_{T}, \rho_{\max } \geq \frac{\Lambda^{*}}{t^{-1}\left(S^{*}\right)}\right\}}\right] \\
& =\log \left(\frac{1}{t^{-1}\left(S^{*}\right)}\right) \operatorname{Pr}\left(\tilde{\mathcal{S}}_{2}\right)=\left(\epsilon_{p}-\epsilon_{p}^{0}\right) \log \left(\frac{1}{t^{-1}\left(S^{*}\right)}\right)
\end{aligned}
$$

Note that the inequality in (B.40) is from the condition $\rho_{\max } \geq \frac{\Lambda^{*}}{t^{-1}\left(S^{*}\right)}$.

Then, we have

$$
\begin{aligned}
\lim _{M \rightarrow \infty} \frac{C_{s}^{*}}{\log (\log M)} & =\lim _{M \rightarrow \infty} \frac{E\left[\log \frac{\rho_{\max }}{\Lambda^{*}} 1_{\left\{g \geq g_{T}, \rho_{\max } \geq \Lambda^{*}\right\}}\right]}{\log (\log M)}+\lim _{M \rightarrow \infty} \frac{E\left[\log \frac{\rho_{\max }}{\Lambda^{*}} 1_{\left.\left\{g \geq g_{T}, \rho_{\max } \geq \frac{\Lambda^{*}}{t^{-1}\left(S^{*}\right)}\right\}\right]}\right.}{\log (\log M)} \\
& =\epsilon_{p}^{0}+\lim _{M \rightarrow \infty} \frac{E\left[\operatorname { l o g } \frac { \rho _ { \operatorname { m a x } } } { \Lambda ^ { * } } 1 \left\{g \geq g_{T}, \rho_{\left.\left.\max \geq \frac{\Lambda^{*}}{t^{-1}\left(S^{*}\right)}\right\}\right]}\right.\right.}{\log (\log M)} \\
& \geq \epsilon_{p}^{0}+\left(\epsilon_{p}-\epsilon_{p}^{0}\right) \lim _{M \rightarrow \infty} \frac{\log \left(\frac{1}{t^{-1}\left(S^{*}\right)}\right)}{\log (\log M)} \\
& =\epsilon_{p}
\end{aligned}
$$

Note that the last equality in (B.41) is from Lemma 3.4.1. Thus, we can show that $\lim _{M \rightarrow \infty} \frac{C_{s}^{*}}{\log (\log M)} \geq \epsilon_{p}$.

Finally, we have that $\lim _{M \rightarrow \infty} \frac{C_{s}^{*}}{\log (\log M)}=\epsilon_{p}$. 


\section{B.17 Proof of Theorem 3.4.2 for $\epsilon_{p}=\epsilon_{p}^{0}$}

In this case, it implies that $\operatorname{Pr}\left(\tilde{\mathcal{S}}_{2}\right)=\epsilon_{p}-\epsilon_{p}^{0}=0$ and $\rho_{\max }=h_{\max }$ in $\tilde{\mathcal{S}}_{1}$. Then, the throughput from the SBS is expressed as $C_{s}^{*}=E\left[\log \left(1+\rho_{\max } P_{O}\right) 1_{\left\{\tilde{\mathcal{S}}_{1}\right\}}\right]=$ $\epsilon_{p}^{0} E\left[\log \left(1+\rho_{\max } P_{O}\right)\right]$. Hence, we can conclude that $\lim _{M \rightarrow \infty} \frac{C_{s}^{*}}{\log (\log M)}=\epsilon_{p}$.

\section{B.18 Proof of Theorem 3.4.2 for $\epsilon_{p}>\epsilon_{p}^{0}$}

In this case, throughput $C_{s}^{*}$ can be described as

$$
C_{s}^{*}=E\left[\log \left(1+\rho_{\max } P_{O}\right) 1_{\left\{g<g_{T}\right\}}\right]+E\left[\log \left(1+\rho_{\max } P_{O}\right) 1_{\left\{g \geq g_{T}, 1+\rho_{\max } P_{O} \geq \frac{1}{t^{-1}\left(S^{*}\right)}\right\}}\right]
$$

As shown previously, $E\left[\log \left(1+\rho_{\max } P_{O}\right) 1_{\left\{g<g_{T}\right\}}\right]$ scales like $\epsilon_{p}^{0} \log (\log M)$. Now, we will show that $E\left[\log \left(1+\rho_{\max } P_{O}\right) 1_{\left\{g \geq g_{T}, 1+\rho_{\max } P_{O} \geq \frac{1}{t^{-1}\left(S^{*}\right)}\right\}}\right]$ scales like

$\left(\epsilon_{p}-\epsilon_{p}^{0}\right) \log (\log M)$.

The upper-bound of $E\left[\log \left(1+\rho_{\max } P_{O}\right) 1_{\left\{g \geq g_{T}, 1+\rho_{\max } P_{O} \geq \frac{1}{t^{-1}\left(S^{*}\right)}\right\}}\right]$ is as follows

$$
\begin{aligned}
& E\left[\log \left(1+\rho_{\max } P_{O}\right) 1_{\left\{g \geq g_{T}, 1+\rho_{\max } P_{O} \geq \frac{1}{t^{-1}\left(S^{*}\right)}\right\}}\right] \\
& =E\left[\log \left(1+\rho_{\max } P_{O}\right) 1_{\left\{1 \leq \rho_{\max } \leq \log M, g \geq g_{T}, 1+\rho_{\max } P_{O} \geq \frac{1}{t^{-1}\left(S^{*}\right)}\right\}}\right] \\
& +\sum_{i=1}^{\infty} E\left[\log \left(1+\rho_{\max } P_{O}\right) 1_{\left\{i \log M \leq \rho_{\max } \leq(i+1) \log M, g \geq g_{T}, 1+\rho_{\max } P_{O} \geq \frac{1}{t^{-1}\left(S^{*}\right)}\right\}}\right] \\
& \leq \log \left(1+P_{O} \log M\right) \operatorname{Pr}\left(1 \leq \rho_{\max } \leq \log M, g \geq g_{T}, 1+\rho_{\max } P_{O} \geq \frac{1}{t^{-1}\left(S^{*}\right)}\right) \\
& +\sum_{i=1}^{\infty} \log \left(1+P_{O}(i+1) \log M\right) \operatorname{Pr}\left(i \log M \leq \rho_{\max } \leq(i+1) \log M, g \geq g_{T}, 1+\rho_{\max } P_{O} \geq \frac{1}{t^{-1}\left(S^{*}\right)}\right) \\
& \leq \log \left(1+P_{O} \log M\right) \operatorname{Pr}\left(g \geq g_{T}, 1+\rho_{\max } P_{O} \geq \frac{1}{t^{-1}\left(S^{*}\right)}\right) \\
& +\sum_{i=1}^{\infty} \log \left(1+P_{O}(i+1) \log M\right) \operatorname{Pr}\left(i \log M \leq \rho_{\max } \leq(i+1) \log M\right) \\
& \stackrel{(a)}{\leq}\left(\epsilon_{p}-\epsilon_{p}^{0}\right) \log \left(1+P_{O} \log M\right)+\sum_{i=1}^{\infty} \log \left(1+P_{O}(i+1) \log M\right) O\left(\frac{e^{O(i)}}{M^{i} O(i)}\right)
\end{aligned}
$$

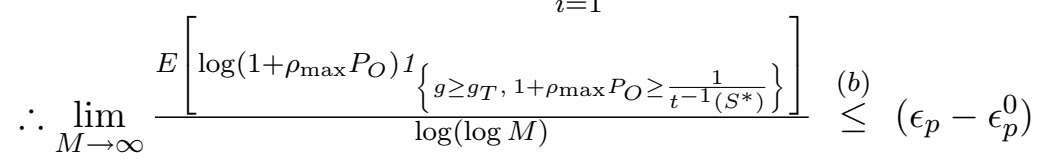

In (B.43), (a) follows the result that $\operatorname{Pr}\left(\rho_{\max } \geq i \log M\right) \leq O\left(\frac{e^{O(i)}}{M^{i} O(i)}\right)$ because $\rho_{\text {max }}$ grows like $\log M$, while $(b)$ is because $\sum_{i=1}^{\infty} \log \left(1+P_{O}(i+1) \log M\right) O\left(\frac{e^{O(i)}}{M^{i} O(i)}\right)$ is finite [81]. 
Also, the lower-bound can be computed as follows.

$$
\begin{aligned}
E\left[\log \left(1+\rho_{\max } P_{O}\right) 1_{\left.\left\{g \geq g_{T}, 1+\rho_{\max } P_{O} \geq \frac{1}{t^{-1}\left(S^{*}\right)}\right\}\right]}\right. & \geq E\left[\log \left(\frac{1}{t^{-1}\left(S^{*}\right)}\right) 1_{\left.\left\{g \geq g_{T}, 1+\rho_{\max } P_{O} \geq \frac{1}{t^{-1}\left(S^{*}\right)}\right\}\right]}\right] \\
& =\log \left(\frac{1}{t^{-1}\left(S^{*}\right)}\right) \operatorname{Pr}\left(g \geq g_{T}, 1+\rho_{\max } P_{O} \geq \frac{1}{t^{-1}\left(S^{*}\right)}\right) \\
& =\left(\epsilon_{p}-\epsilon_{p}^{0}\right) \log \left(\frac{1}{t^{-1}\left(S^{*}\right)}\right)
\end{aligned}
$$

By Lemma 3.4.1, it reveals that $\frac{1}{t^{-1}\left(S^{*}\right)}=\Theta(\log M)$, we then apply the facts that $E\left[\log \left(1+\rho_{\max } P_{O}\right) 1_{\left\{g<g_{T}\right\}}\right]$ scales like $\epsilon_{p}^{0} \log (\log M)$ and the results from (B.43) and (B.44). Thus, it is not hard to prove that $\lim _{M \rightarrow \infty} \frac{C_{s}^{*}}{\log (\log M)}$ is upper-bounded and lower-bounded by $\epsilon_{p}$, i.e. $\lim _{M \rightarrow \infty} \frac{C_{s}^{*}}{\log (\log M)}=\epsilon_{p}$. 


\section{Appendix C}

\section{Proofs in Chapter 4}

\section{C.1 Proof of Lemma 4.2.1}

We first show that for an arbitrary feasible probabilistic power scheme $\mathbf{P}(\boldsymbol{\nu})$, we can always construct another feasible scheme $\mathbf{P}^{\prime}(\boldsymbol{\nu})$ which is randomized among deterministic power schemes $\mathbf{p}_{k}(\boldsymbol{\nu})$ with time-sharing factors $w_{k}(\boldsymbol{\nu}), k=1,2$, and performs equally well or better than $\mathbf{P}(\boldsymbol{\nu})$.

By the concavity of $r_{s}(\boldsymbol{\nu}, \mathbf{p}(\boldsymbol{\nu}))$ over $\mathbf{p}$ and Jensen's inequality, we obtain

$$
\begin{aligned}
& r_{s}\left(\boldsymbol{\nu}, \mathbf{p}_{1}(\boldsymbol{\nu})\right) \geq E\left[r_{s}\left(\boldsymbol{\nu}, \mathbf{P}_{s}(\boldsymbol{\nu})\right) \mid r_{p}(\boldsymbol{\nu}, \mathbf{p}(\boldsymbol{\nu})) \geq r_{p}^{0}, \boldsymbol{\nu}\right] \\
& r_{s}\left(\boldsymbol{\nu}, \mathbf{p}_{2}(\boldsymbol{\nu})\right) \geq E\left[r_{s}\left(\boldsymbol{\nu}, \mathbf{P}_{s}(\boldsymbol{\nu})\right) \mid r_{p}(\boldsymbol{\nu}, \mathbf{p}(\boldsymbol{\nu}))<r_{p}^{0}, \boldsymbol{\nu}\right]
\end{aligned}
$$

Further, the set of all possible $\mathbf{p}(\boldsymbol{\nu})$ such that $r_{p}(\boldsymbol{\nu}, \mathbf{p}(\boldsymbol{\nu})) \geq r_{p}^{0}$ lies in the halfspace defined by $\mathbf{p}^{T}(\boldsymbol{\nu}) \boldsymbol{\beta} \leq \mathcal{P}_{p}$. Due to the definition in (4.8), $\mathbf{p}_{1}(\boldsymbol{\nu})$ must also be in that halfspace and it implies that $\mathbf{p}_{1}^{T}(\boldsymbol{\nu}) \boldsymbol{\beta} \leq \mathcal{P}_{p}$. Also, note that if $w_{1}(\boldsymbol{\nu})=0$, the constraint is discarded from the optimization problem. Thus, we can write

$$
w_{1}(\boldsymbol{\nu})\left[\mathcal{P}_{p}(g)-\boldsymbol{\beta}^{T} \mathbf{p}_{1}(\boldsymbol{\nu})\right] \geq 0
$$

Construct the new probabilistic scheme $\mathbf{P}^{\prime}$ such that $\mathbf{P}^{\prime}=\mathbf{p}_{1}(\boldsymbol{\nu})$ with probability $w_{1}(\boldsymbol{\nu})$ and $\mathbf{P}^{\prime}=\mathbf{p}_{2}(\boldsymbol{\nu})$ with probability $w_{2}(\boldsymbol{\nu})=1-w_{1}(\boldsymbol{\nu})$.

For the PU's outage probability based on the policy $\mathbf{P}^{\prime}$, we can show that $\operatorname{Pr}\left\{r_{p}\left(\boldsymbol{\nu}, \mathbf{P}^{\prime}(\nu)\right) \geq r_{p}^{0} \mid \boldsymbol{\nu}\right\} \geq w_{1}(\boldsymbol{\nu})$, so $E\left[w_{1}(\boldsymbol{\nu})\right] \geq 1-\epsilon_{p}$.

The average power of $\mathbf{P}^{\prime}$ does not exceed $\mathbf{P}_{a v}$, since

$$
E\left[\mathbf{P}^{\prime}(\boldsymbol{\nu})\right]=E\left[w_{1}(\boldsymbol{\nu}) \mathbf{p}_{1}(\boldsymbol{\nu})+\left(1-w_{1}(\boldsymbol{\nu})\right) \mathbf{p}_{2}(\boldsymbol{\nu})\right]=E[\mathbf{P}(\boldsymbol{\nu})] \preceq \mathbf{P}_{a v}
$$


Now, it can be proved that $\mathbf{P}^{\prime}(\boldsymbol{\nu})$ achieves higher average SU rate as follows:

$$
\begin{aligned}
E\left[r_{s}\left(\boldsymbol{\nu}, \mathbf{P}^{\prime}(\boldsymbol{\nu})\right)\right] & =E\left[w_{1}(\boldsymbol{\nu}) r_{s}\left(\boldsymbol{\nu}, \mathbf{p}_{1}(\boldsymbol{\nu})\right)+\left(1-w_{1}(\boldsymbol{\nu})\right) r_{s}\left(\boldsymbol{\nu}, \mathbf{p}_{2}(\boldsymbol{\nu})\right)\right] \\
& \stackrel{(a)}{\geq} E\left[r_{s}(\boldsymbol{\nu}, \mathbf{P}(\boldsymbol{\nu}))\right]
\end{aligned}
$$

where the inequality is due to Jensen's inequality.

\section{C.2 KKT conditions corresponding to Problem $(4.9)$}

Let $i \in\{1,2, \ldots, M\}$ and $k \in\{1,2\}$. From the Karush-Kuhn-Tucker (KKT) conditions, we have

$$
\begin{aligned}
& \frac{\partial l(\ldots)}{\partial p_{k, i}^{*}(\boldsymbol{\nu})} \begin{cases}=0, & p_{k, i}^{*}(\boldsymbol{\nu})>0 \\
\leq 0, & p_{k, i}^{*}(\boldsymbol{\nu})=0\end{cases} \\
& \frac{\partial l(\ldots)}{\partial w_{1}^{*}(\boldsymbol{\nu})}\left\{\begin{array}{l}
=0, \quad 0<w_{1}^{*}(\boldsymbol{\nu})<1 \\
\leq 0, \quad w_{1}^{*}(\boldsymbol{\nu})=0 \\
\geq 0, \quad w_{1}^{*}(\boldsymbol{\nu})=1
\end{array}\right. \\
& \lambda^{* T}\left(\mathbf{P}_{a v}-E\left[w_{1}(\boldsymbol{\nu}) \mathbf{p}_{1}(\boldsymbol{\nu})+\left(1-w_{1}(\boldsymbol{\nu})\right) \mathbf{p}_{2}(\boldsymbol{\nu})\right]\right)=0 \\
& s^{*}\left(E\left[w_{1}^{*}(\boldsymbol{\nu})\right]-\left(1-\epsilon_{p}\right)\right)=0 \\
& \tau^{*}(\boldsymbol{\nu}) w_{1}^{*}(\boldsymbol{\nu})\left[\mathcal{P}_{p}(g)-\boldsymbol{\beta}^{T} \mathbf{p}_{1}^{*}(\boldsymbol{\nu})\right]=0 \\
& \mathbf{p}_{k}^{*}(\boldsymbol{\nu}) \succeq 0, \quad k=1,2 \\
& 0 \leq w_{k}^{*}(\boldsymbol{\nu}) \leq 1
\end{aligned}
$$

By using the KKT conditions from (C.5) to (C.11), we can derive $\mathbf{p}_{2}^{*}(\boldsymbol{\nu}), \mathbf{p}_{1}^{*}(\boldsymbol{\nu})$, and $w_{1}^{*}(\boldsymbol{\nu})$

\section{C.3 Proof of Lemma 4.2.3}

We will show that, by $\mathbf{P}^{\prime}(\boldsymbol{\nu})$, we can achieve a larger capacity region, i.e. $\mathcal{C}_{M A C}(\boldsymbol{\nu}, \mathbf{P}(\boldsymbol{\nu})) \subseteq$ $\mathcal{C}_{M A C}\left(\boldsymbol{\nu}, \mathbf{P}^{\prime}(\boldsymbol{\nu})\right)$ 
Since for all $S$,

$$
\begin{aligned}
& E\left[\log \left(1+\sum_{i \in S} \bar{h}_{i} P_{i}^{\prime}(\boldsymbol{\nu})\right)\right] \\
& =E\left[w_{1}(\boldsymbol{\nu}) \log \left(1+\sum_{i \in S} \bar{h}_{i} p_{1, i}(\boldsymbol{\nu})\right)+\left(1-w_{1}(\boldsymbol{\nu})\right) \log \left(1+\sum_{i \in S} \bar{h}_{i} p_{2, i}(\boldsymbol{\nu})\right)\right] \\
& \geq E\left[\log \left(1+\sum_{i \in S} \bar{h}_{i} P_{i}(\boldsymbol{\nu})\right)\right]
\end{aligned}
$$

where the inequality follows from Jensen's inequality. Therefore, $\mathcal{C}_{M A C}(\boldsymbol{\nu}, \mathbf{P}(\boldsymbol{\nu})) \subseteq$ $\mathcal{C}_{M A C}\left(\boldsymbol{\nu}, \mathbf{P}^{\prime}(\boldsymbol{\nu})\right)$.

From the definition in (4.19), it is also evident that $\mathbf{r}_{k} \in \mathcal{C}_{M A C}\left(\boldsymbol{\nu}, \mathbf{p}_{k}(\boldsymbol{\nu})\right)$. Note that the policy also satisfies AIPC and POC and this can be proven by a similar procedure as used in Appendix C.1.

\section{C.4 KKT conditions corresponding to Problem}

$(4.20)$

$$
\begin{gathered}
\frac{\partial l(\ldots)}{\partial p_{k, i}^{*}(\boldsymbol{\nu})}\left\{\begin{array}{c}
=0, \quad p_{k, i}^{*}(\boldsymbol{\nu})>0 \\
\leq 0, \quad p_{k, i}^{*}(\boldsymbol{\nu})=0
\end{array}, \quad k=1,2 .\right. \\
\frac{\partial l(\ldots)}{\partial w_{1}^{*}(\boldsymbol{\nu})}\left\{\begin{array}{l}
\geq 0, \quad w_{1}^{*}(\boldsymbol{\nu})=1 \\
=0, \quad 0<w_{1}^{*}(\boldsymbol{\nu})<1 \\
\leq 0, \quad w_{1}^{*}(\boldsymbol{\nu})=0
\end{array}\right. \\
\lambda^{* T}\left(\mathbf{P}_{a v}-E\left[w_{1}^{*}(\boldsymbol{\nu}) \mathbf{p}_{1}^{*}(\boldsymbol{\nu})+\left(1-w_{1}^{*}(\boldsymbol{\nu})\right) \mathbf{p}_{2}^{*}(\boldsymbol{\nu})\right]\right)=0 \\
s^{*}\left(E\left[w_{1}^{*}(\boldsymbol{\nu})\right]-\left(1-\epsilon_{p}\right)\right)=0 \\
\tau^{*}(\boldsymbol{\nu}) w_{1}^{*}(\boldsymbol{\nu})\left(\mathcal{P}_{p}(g)-\boldsymbol{\beta}^{T} \mathbf{p}_{1}^{*}(\boldsymbol{\nu})\right)=0 \\
\mathbf{p}_{k}^{*}(\boldsymbol{\nu}) \succeq \mathbf{0}, k=1,2 \\
0 \leq w_{1}^{*}(\boldsymbol{\nu}) \leq 1 \\
\mathbf{r}_{k}^{*} \in \mathcal{C}_{M A C}\left(\boldsymbol{\nu}, \mathbf{p}_{k}^{*}(\boldsymbol{\nu})\right), k=1,2 .
\end{gathered}
$$




\section{C.5 Proof of Lemma 4.3.1}

For an arbitrary feasible probabilistic power scheme $\mathbf{P}(\boldsymbol{\nu})$, we can always construct another feasible scheme $\mathbf{P}^{\prime}(\boldsymbol{\nu})$ which is randomized among deterministic power schemes $\mathbf{p}_{k}(\boldsymbol{\nu})$ with time-sharing factors $\psi_{k}(\boldsymbol{\nu}), k=1,2,3$, and performs equally well or better than $\mathbf{P}(\boldsymbol{\nu})$.

As $\operatorname{Pr}\left\{\mathbf{P}^{\prime}(\boldsymbol{\nu})=E\left[\mathbf{P}(\boldsymbol{\nu}) \mid \mathbf{r}_{o} \in \mathcal{C}_{M A C}(\boldsymbol{\nu}, \mathbf{p}(\boldsymbol{\nu})), \boldsymbol{\nu}\right] \mid \boldsymbol{\nu}\right\}=\psi_{1}(\boldsymbol{\nu})+\psi_{2}(\boldsymbol{\nu})$, it implies that $\operatorname{Pr}\left\{\mathbf{r}_{o} \in \mathcal{C}_{M A C}\left(\boldsymbol{\nu}, \mathbf{P}^{\prime}(\boldsymbol{\nu})\right)\right\}=E\left[\operatorname{Pr}\left\{\mathbf{r}_{o} \in \mathcal{C}_{M A C}\left(\boldsymbol{\nu}, \mathbf{P}^{\prime}(\boldsymbol{\nu})\right) \mid \boldsymbol{\nu}\right\}\right]=E\left[\psi_{1}(\boldsymbol{\nu})+\psi_{2}(\boldsymbol{\nu})\right] \geq$ $1-\epsilon_{s}$.

For ATPC, it is obvious that $E\left[\mathbf{P}^{\prime}(\boldsymbol{\nu})\right]=E\left[\sum_{k=1}^{4} \psi_{k}(\boldsymbol{\nu}) \mathbf{p}_{k}(\boldsymbol{\nu})\right]=E[\mathbf{P}(\boldsymbol{\nu})] \preceq \mathbf{P}_{a v}$.

Now, we will show that $r_{p}\left(\boldsymbol{\nu}, E\left[\mathbf{P}(\boldsymbol{\nu}) \mid r_{p}(\boldsymbol{\nu}, \mathbf{p}(\boldsymbol{\nu})) \geq r_{p}^{0}, \boldsymbol{\nu}\right]\right) \geq r_{p}^{0}$. Note that when $r_{p}(\boldsymbol{\nu}, \mathbf{p}(\boldsymbol{\nu})) \geq r_{p}^{0}$, it implies $\boldsymbol{\beta}^{T} \mathbf{p}(\boldsymbol{\nu}) \leq \mathcal{P}_{p}(g)$, so the possible solution lies in a halfspace. Further, notice that

$E\left[\mathbf{P}(\boldsymbol{\nu}) \mid r_{p}(\boldsymbol{\nu}, \mathbf{p}(\boldsymbol{\nu})) \geq r_{p}^{0}, \mathbf{r}_{o} \in \mathcal{C}_{M A C}(\boldsymbol{\nu}, \mathbf{p}(\boldsymbol{\nu})), \boldsymbol{\nu}\right]$ is a convex combination of all possible solutions that satisfy $\mathbf{r}_{o} \in \mathcal{C}_{M A C}(\boldsymbol{\nu}, \mathbf{p}(\boldsymbol{\nu}))$ and $r_{p}(\boldsymbol{\nu}, \mathbf{p}(\boldsymbol{\nu})) \geq r_{p}^{0}$. Hence, it implies that $r_{p}^{0} \leq r_{p}\left(\boldsymbol{\nu}, \mathbf{p}_{1}(\boldsymbol{\nu})\right)$. In other words, we can write $\psi_{1}(\boldsymbol{\nu})\left(\mathcal{P}_{p}(g)-\boldsymbol{\beta}^{T} \mathbf{p}_{1}(\boldsymbol{\nu})\right) \geq$ 0.

Likewise, we can also write $\psi_{3}(\boldsymbol{\nu})\left(\mathcal{P}_{p}(g)-\boldsymbol{\beta}^{T} \mathbf{p}_{3}(\boldsymbol{\nu})\right) \geq 0$ due to the same reason.

For POC, since $\operatorname{Pr}\left\{\mathbf{P}^{\prime}(\boldsymbol{\nu})=E\left[\mathbf{P}(\boldsymbol{\nu}) \mid r_{p}(\boldsymbol{\nu}, \mathbf{p}(\boldsymbol{\nu})) \geq r_{p}^{0}, \boldsymbol{\nu}\right] \mid \boldsymbol{\nu}\right\}=\psi_{1}(\boldsymbol{\nu})+\psi_{3}(\boldsymbol{\nu})$, $\operatorname{Pr}\left\{r_{p}\left(\boldsymbol{\nu}, \mathbf{P}^{\prime}(\boldsymbol{\nu})\right)\right\}=E\left[\operatorname{Pr}\left\{r_{p}\left(\boldsymbol{\nu}, \mathbf{P}^{\prime}(\boldsymbol{\nu})\right) \mid \boldsymbol{\nu}\right\}\right]=E\left[\psi_{1}(\boldsymbol{\nu})+\psi_{3}(\boldsymbol{\nu})\right] \geq 1-\epsilon_{p}$.

Also, by the definition in (4.38), we have $\mathbf{r}_{o} \in \mathcal{C}_{M A C}\left(\boldsymbol{\nu}, \mathbf{p}_{1}(\boldsymbol{\nu})\right)$. Likewise, $\mathbf{r}_{o} \in$ $\mathcal{C}_{M A C}\left(\boldsymbol{\nu}, \mathbf{p}_{2}(\boldsymbol{\nu})\right)$

We can then show that $\mathcal{C}_{M A C}(\boldsymbol{\nu}, \mathbf{P}(\boldsymbol{\nu})) \subseteq \mathcal{C}_{M A C}\left(\boldsymbol{\nu}, \mathbf{P}^{\prime}(\boldsymbol{\nu})\right)$ because of the concavity of the $\log$ function in the definition of $\mathcal{C}_{M A C}(\boldsymbol{\nu}, \mathbf{P}(\boldsymbol{\nu}))$.

\section{C.6 Proof of convexity of the set $\mathcal{Q}_{C}\left(\mathbf{r}_{o}\right)$}

For a given rate vector $\mathbf{r}_{o}$, consider the two points $\left(\bar{\varepsilon}_{s}^{(1)}, \varepsilon_{p}^{(1)}, \overline{\mathbf{P}}^{(1)}\right)$ and $\left(\bar{\varepsilon}_{s}^{(2)}, \varepsilon_{p}^{(2)}, \overline{\mathbf{P}}^{(2)}\right)$ in the set $\mathcal{Q}_{C}\left(\mathbf{r}_{o}\right)$. By Definition 4.3.1 and Lemma 4.3.1, there must exist power allocation policies $\mathbf{P}^{(1)}(\boldsymbol{\nu})$ and $\mathbf{P}^{(2)}(\boldsymbol{\nu})$ in the form expressed in Lemma 4.3.1 such that, for all $l=1,2, \bar{\varepsilon}_{s}^{(l)}=E\left[\psi_{1}^{(l)}(\boldsymbol{\nu})+\psi_{2}^{(l)}(\boldsymbol{\nu})\right], 1-\varepsilon_{p}^{(l)}=E\left[\psi_{1}^{(l)}(\boldsymbol{\nu})+\psi_{3}^{(l)}(\boldsymbol{\nu})\right]$ and 
$\overline{\mathbf{P}}^{(l)}=E\left[\mathbf{P}^{(l)}(\boldsymbol{\nu})\right]=E\left[\sum_{k=1}^{4} \psi_{k}^{(l)}(\boldsymbol{\nu}) \mathbf{p}_{k}^{(l)}\right]$. To prove convexity of $\mathcal{Q}_{C}\left(\mathbf{r}_{o}\right)$, we must show that $\left(\delta \bar{\varepsilon}_{s}^{(1)}+(1-\delta) \bar{\varepsilon}_{s}^{(2)}, \delta \varepsilon_{p}^{(1)}+(1-\delta) \varepsilon_{p}^{(2)}, \delta \overline{\mathbf{P}}^{(1)}+(1-\delta) \overline{\mathbf{P}}^{(2)}\right) \in \mathcal{Q}_{C}\left(\mathbf{r}_{o}\right)$, where $\delta \in[0,1]$. To do so, we define another random power allocation $\mathbf{P}^{(x)}(\boldsymbol{\nu})$ that is randomized using the power policy $\mathbf{P}^{(1)}(\boldsymbol{\nu})$ with probability $\delta$ and the policy $\mathbf{P}^{(2)}(\boldsymbol{\nu})$ with probability $1-\delta$. Obviously, $\operatorname{Pr}\left\{\mathbf{r}_{o} \in \mathcal{C}_{M A C}\left(\boldsymbol{\nu}, \mathbf{P}^{(x)}(\boldsymbol{\nu})\right)\right\}=E\left[\delta\left(\psi_{1}^{(1)}(\boldsymbol{\nu})+\right.\right.$ $\left.\left.\psi_{2}^{(1)}(\boldsymbol{\nu})\right)+(1-\delta)\left(\psi_{1}^{(2)}(\boldsymbol{\nu})+\psi_{2}^{(2)}(\boldsymbol{\nu})\right)\right]=\delta \bar{\varepsilon}_{s}^{(1)}+(1-\delta) \bar{\varepsilon}_{s}^{(2)}, \operatorname{Pr}\left\{r_{p}\left(\boldsymbol{\nu}, \mathbf{P}^{(x)}(\boldsymbol{\nu})\right) \geq r_{p}^{0}\right\}=$ $E\left[\delta\left(\psi_{1}^{(1)}(\boldsymbol{\nu})+\psi_{3}^{(1)}(\boldsymbol{\nu})\right)+(1-\delta)\left(\psi_{1}^{(2)}(\boldsymbol{\nu})+\psi_{3}^{(2)}(\boldsymbol{\nu})\right)\right]=\delta\left[1-\varepsilon_{p}^{(1)}\right]+(1-\delta)\left[1-\varepsilon_{p}^{(2)}\right]$ and $E\left[\mathbf{P}^{(x)}(\boldsymbol{\nu})\right]=\delta \overline{\mathbf{P}}^{(1)}+(1-\delta) \overline{\mathbf{P}}^{(2)}$. Thus, we have $\left(\delta \bar{\varepsilon}_{s}^{(1)}+(1-\delta) \bar{\varepsilon}_{s}^{(2)}, \delta \varepsilon_{p}^{(1)}+(1-\right.$ $\left.\delta) \varepsilon_{p}^{(2)}, \delta \overline{\mathbf{P}}^{(1)}+(1-\delta) \overline{\mathbf{P}}^{(2)}\right) \in \mathcal{Q}_{C}\left(\mathbf{r}_{o}\right)$ and $\mathcal{Q}_{C}\left(\mathbf{r}_{o}\right)$ is convex.

\section{C.7 KKT conditions corresponding to Problem}

\section{(4.43)}

From the associated (necessary and sufficient) KKT conditions, we have

$$
\begin{gathered}
\frac{\partial l(\ldots)}{\partial p_{k, i}^{*}(\boldsymbol{\nu})}\left\{\begin{array}{c}
=0, \quad p_{k, i}^{*}(\boldsymbol{\nu})>0 \\
\leq 0, \quad p_{k, i}^{*}(\boldsymbol{\nu})=0
\end{array}, \quad k=1,2,3,4 .\right. \\
\frac{\partial l(\ldots)}{\partial \psi_{k}^{*}(\boldsymbol{\nu})}\left\{\begin{array}{c}
=0, \quad \psi_{k}^{*}(\boldsymbol{\nu}) \geq 0 \\
\leq 0, \quad \psi_{k}^{*}(\boldsymbol{\nu})=0
\end{array}, \quad k=1,2,3,4 .\right. \\
\lambda^{* T}\left(\mathbf{P}_{a v}-E\left[w_{1}^{*}(\boldsymbol{\nu}) \mathbf{p}_{1}^{*}(\boldsymbol{\nu})+\left(1-w_{1}^{*}(\boldsymbol{\nu})\right) \mathbf{p}_{2}^{*}(\boldsymbol{\nu})\right]\right)=0 \\
s^{*}\left(E\left[\psi_{1}^{*}(\boldsymbol{\nu})+\psi_{3}^{*}(\boldsymbol{\nu})\right]-\left(1-\epsilon_{p}\right)\right)=0 \\
\varphi_{k}^{*}(\boldsymbol{\nu}) \psi_{k}^{*}(\boldsymbol{\nu})\left(\mathcal{P}_{p}(g)-\boldsymbol{\beta}^{T} \mathbf{p}_{k}^{*}(\boldsymbol{\nu})\right)=0, \quad k=1,3 \\
\Gamma^{*}(\boldsymbol{\nu})\left[1-\sum_{k=1}^{4} \psi_{k}^{*}(\boldsymbol{\nu})\right]=0 \\
\mathbf{p}_{k}^{*}(\boldsymbol{\nu}) \succeq \mathbf{0}, k=1,2,3,4 \\
\psi_{k}^{*}(\boldsymbol{\nu}) \geq 0, k=1,2,3,4 \\
\mathbf{r}_{o} \in \mathcal{C}_{M A C}\left(\boldsymbol{\nu}, \mathbf{p}_{k}^{*}(\boldsymbol{\nu})\right), k=1,2 .
\end{gathered}
$$




\section{C.8 Proof of Proposition 4.3.2}

Note that $\Gamma^{*}(\boldsymbol{\nu}), s^{*}$ and $\varphi_{k}^{*}(\boldsymbol{\nu}) P_{p}(g)$ are independent of $\mathbf{p}_{k}(\boldsymbol{\nu})$. Thus, subproblems (4.45) to (4.48) can be rewritten as follows:

$$
\text { Subproblem for } \mathbf{p}_{4}^{*}: \min _{\mathbf{p}_{4}(\boldsymbol{\nu})} \lambda^{* T} \mathbf{p}_{4}(\boldsymbol{\nu}) \text {. }
$$

$$
\begin{aligned}
\text { Subproblem for } \mathbf{p}_{3}^{*}: & \min _{\mathbf{p}_{3}(\boldsymbol{\nu})}\left[\boldsymbol{\lambda}^{* T}+\varphi^{*}(\boldsymbol{\nu}) \boldsymbol{\beta}^{T}\right] \mathbf{p}_{3}(\boldsymbol{\nu}) \\
& \text { s.t. } \varphi_{3}^{*}(\boldsymbol{\nu})\left[P_{p}(g)-\boldsymbol{\beta}^{T} \mathbf{p}_{3}(\boldsymbol{\nu})\right]=0 .
\end{aligned}
$$

$$
\begin{aligned}
\text { Subproblem for } \mathbf{p}_{2}^{*}: & \min _{\mathbf{p}_{2}(\boldsymbol{\nu})} \lambda^{* T} \mathbf{p}_{2}(\boldsymbol{\nu}) \\
& \text { s.t. } \mathbf{r}_{o} \in \mathcal{C}_{M A C}\left(\boldsymbol{\nu}, \mathbf{p}_{2}(\boldsymbol{\nu})\right) .
\end{aligned}
$$

$$
\begin{aligned}
\text { Subproblem for } \mathbf{p}_{1}^{*}: & \min _{\mathbf{p}_{1}(\boldsymbol{\nu})}\left[\boldsymbol{\lambda}^{* T}+\varphi_{1}^{*}(\boldsymbol{\nu}) \boldsymbol{\beta}^{T}\right] \mathbf{p}_{1}(\boldsymbol{\nu}) \\
\text { s.t. } & \mathbf{r}_{o} \in \mathcal{C}_{M A C}\left(\boldsymbol{\nu}, \mathbf{p}_{1}(\boldsymbol{\nu})\right), \\
& \varphi_{1}^{*}(\boldsymbol{\nu})\left[P_{p}(g)-\boldsymbol{\beta}^{T} \mathbf{p}_{1}(\boldsymbol{\nu})\right]=0 .
\end{aligned}
$$

From (C.30) and (C.31), it is obvious that $\mathbf{p}_{4}^{*}(\boldsymbol{\nu})=\mathbf{p}_{3}^{*}(\boldsymbol{\nu})=\mathbf{0}$. Now, Lemma 4.3.2 can be applied to solve for $\mathbf{p}_{2}^{*}(\boldsymbol{\nu})$ and $\mathbf{p}_{1}^{*}(\boldsymbol{\nu})$. For a given rate vector $\mathbf{r}_{o}$ and channel state $\boldsymbol{\nu}$, we define the set of received powers that can support $\mathbf{r}_{o}, \tilde{\mathscr{G}}\left(\mathbf{r}_{o}^{*}\right)$, as follows:

$$
\tilde{\mathscr{G}}\left(\mathbf{r}_{o}^{*}\right)=\left\{\mathbf{q}: q_{i}=\bar{h}_{i} p_{i}, \mathbf{r}_{o} \in \mathcal{C}_{M A C}(\boldsymbol{\nu}, \mathbf{p})\right\}
$$

which is a contra-polymatroid with rank function: $f(S)=\exp \left(\mathbf{r}_{o}(S)\right)-1$.

Substitute each $p_{k, i}$ by $\frac{q_{k, i}}{h_{i}}$ and utilize Lemma 4.3.2 above. Thus, the solution for $\mathbf{p}_{2}^{*}$ is as follows:

$$
p_{2, \pi(i)}^{*}= \begin{cases}\frac{1}{h_{\pi(i)}}\left[\exp \left(r_{o, \pi(i)}\right)-1\right], & i=1 \\ \frac{1}{h_{\pi(i)}}\left[\exp \left(\sum_{m=1}^{i} r_{o, \pi(m)}\right)-\exp \left(\sum_{m=1}^{i-1} r_{o, \pi(m)}\right)\right], & i=2, \ldots, M\end{cases}
$$

where the permutation $\pi$ satisfies $\frac{\lambda_{\pi(1)}^{*}}{h_{\pi(1)}} \geq \ldots \geq \frac{\lambda_{\pi(M)}^{*}}{\bar{h}_{\pi(M)}}$. For $\mathbf{p}_{1}^{*}$, we have

$$
p_{1, \pi(i)}^{*}= \begin{cases}\frac{1}{\bar{h}_{\pi(i)}}\left[\exp \left(r_{o, \pi(i)}\right)-1\right], & i=1 \\ \frac{1}{h_{\pi(i)}}\left[\exp \left(\sum_{m=1}^{i} r_{o, \pi(m)}\right)-\exp \left(\sum_{m=1}^{i-1} r_{o, \pi(m)}\right)\right], & i=2, \ldots, M\end{cases}
$$




$$
\varphi_{1}^{*}(\boldsymbol{\nu})\left[\boldsymbol{\beta}^{T} \mathbf{p}_{1}^{*}-\mathcal{P}_{p}(g)\right]=0
$$

where the permutation $\pi$ satisfies $\frac{\eta_{\pi(1)}}{h_{\pi(1)}} \geq \ldots \geq \frac{\eta_{\pi(M)}}{h_{\pi(M)}}$.

\section{C.9 Proof of Lemma 4.3.3}

For an arbitrary feasible probabilistic power scheme $\mathbf{P}(\boldsymbol{\nu})$, we can always construct another feasible scheme $\mathbf{P}^{\prime}(\boldsymbol{\nu})$ which is randomized among deterministic power schemes $\mathbf{p}_{k}(\boldsymbol{\nu})$ with time-sharing factors $\psi_{k}(\boldsymbol{\nu})$, and performs equally well or better than $\mathbf{P}(\boldsymbol{\nu})$.

Since $\operatorname{Pr}\left\{\mathbf{P}^{\prime}(\boldsymbol{\nu})=E\left[\mathbf{P}(\boldsymbol{\nu}) \mid \mathscr{S}_{n}(j), \boldsymbol{\nu}\right] \mid \boldsymbol{\nu}\right\}=\zeta_{1}(\boldsymbol{\nu}, j)+\zeta_{2}(\boldsymbol{\nu}, j)$, it means that $\operatorname{Pr}\left\{r_{i}(\boldsymbol{\nu}, \mathbf{P}(\boldsymbol{\nu})) \geq r_{o, i}\right\}=\sum_{j: i \in \mathscr{S}_{n}(j)} E\left[\operatorname{Pr}\left\{\mathscr{S}_{n}(j)\right\}\right]=\sum_{j: i \in \mathscr{S}_{n}(j)} E\left[\zeta_{1}(\boldsymbol{\nu}, j)+\zeta_{2}(\boldsymbol{\nu}, j)\right] \geq$ $1-\epsilon_{s, i}$ for all $i$.

For ATPC, it is obvious that $E\left[\mathbf{P}^{\prime}(\boldsymbol{\nu})\right]=E\left[\sum_{j=0}^{2^{M}-1} \sum_{k=1}^{2} \zeta_{k}(\boldsymbol{\nu}, j) \mathbf{p}_{k}(\boldsymbol{\nu}, j)\right]=E[\mathbf{P}(\boldsymbol{\nu})] \preceq$ $\mathbf{P}_{a v}$.

Now, we will show that $r_{p}\left(\boldsymbol{\nu}, E\left[\mathbf{P}(\boldsymbol{\nu}) \mid r_{p}(\boldsymbol{\nu}, \mathbf{p}(\boldsymbol{\nu})) \geq r_{p}^{0}, \mathscr{S}_{n}(j), \boldsymbol{\nu}\right]\right) \geq r_{p}^{0}$. Note that when $r_{p}(\boldsymbol{\nu}, \mathbf{p}(\boldsymbol{\nu})) \geq r_{p}^{0}$, it implies $\boldsymbol{\beta}^{T} \mathbf{p}(\boldsymbol{\nu}) \leq \mathcal{P}_{p}(g)$, so the possible solution lies in a halfspace. Further, notice that

$E\left[\mathbf{P}(\boldsymbol{\nu}) \mid r_{p}(\boldsymbol{\nu}, \mathbf{p}(\boldsymbol{\nu})) \geq r_{p}^{0}, \mathscr{S}_{n}(j), \boldsymbol{\nu}\right]$ is a convex combination of all possible solutions in that halfspace weighted by the probability of each solution. Hence, $E\left[\mathbf{P}(\boldsymbol{\nu}) \mid r_{p}(\boldsymbol{\nu}, \mathbf{p}(\boldsymbol{\nu})) \geq r_{p}^{0}, \mathscr{S}_{n}(j), \boldsymbol{\nu}\right]$ also lies in the same halfspace due to convex set properties, which means $\zeta_{1}(\boldsymbol{\nu}, j)\left(\mathcal{P}_{p}(g)-\boldsymbol{\beta}^{T} \mathbf{p}_{1}(\boldsymbol{\nu}, j)\right) \geq 0$.

For POC, since $\operatorname{Pr}\left\{\mathbf{P}^{\prime}(\boldsymbol{\nu})=E\left[\mathbf{P}(\boldsymbol{\nu}) \mid r_{p}(\boldsymbol{\nu}, \mathbf{p}(\boldsymbol{\nu})) \geq r_{p}^{0}, \boldsymbol{\nu}\right] \mid \boldsymbol{\nu}\right\}=\sum_{j=0}^{2^{M}-1} \sum_{k=1}^{2} \zeta_{1}(\boldsymbol{\nu}, j)$, $\operatorname{Pr}\left\{r_{p}\left(\boldsymbol{\nu}, \mathbf{P}^{\prime}(\boldsymbol{\nu})\right)\right\}=E\left[\operatorname{Pr}\left\{r_{p}\left(\boldsymbol{\nu}, \mathbf{P}^{\prime}(\boldsymbol{\nu})\right) \mid \boldsymbol{\nu}\right\}\right]=\sum_{j=0}^{2^{M}-1} E\left[\zeta_{1}(\boldsymbol{\nu}, j)\right] \geq 1-\epsilon_{p}$

Also, we have the following constraints on $\mathbf{p}_{1}(\boldsymbol{\nu}, j)$ and $\mathbf{p}_{2}(\boldsymbol{\nu}, j)$, i.e. $\boldsymbol{\xi}(j) \mathbf{r}_{o} \in$ $\mathcal{C}_{M A C}\left(\boldsymbol{\nu}, \mathbf{p}_{1}(\boldsymbol{\nu}, j)\right)$ and $\boldsymbol{\xi}(j) \mathbf{r}_{o} \in \mathcal{C}_{M A C}\left(\boldsymbol{\nu}, \mathbf{p}_{2}(\boldsymbol{\nu}, j)\right)$ by the definition.

We can then show that $\mathcal{C}_{M A C}(\boldsymbol{\nu}, \mathbf{P}(\boldsymbol{\nu})) \subseteq \mathcal{C}_{M A C}\left(\boldsymbol{\nu}, \mathbf{P}^{\prime}(\boldsymbol{\nu})\right)$ because of the concavity of the $\log$ function in the definition of $\mathcal{C}_{M A C}(\boldsymbol{\nu}, \mathbf{P}(\boldsymbol{\nu}))$. 


\section{C.10 Proof of convexity of the set $\mathcal{Q}_{I}\left(\mathbf{r}_{o}\right)$}

For a given rate vector $\mathbf{r}_{o}$, consider the two points $\left(\bar{\varepsilon}_{s}^{(1)}, \varepsilon_{p}^{(1)}, \overline{\mathbf{P}}^{(1)}\right)$ and $\left(\overline{\boldsymbol{\varepsilon}}_{s}^{(2)}, \varepsilon_{p}^{(2)}, \overline{\mathbf{P}}^{(2)}\right)$ in the set $\mathcal{Q}_{I}\left(\mathbf{r}_{o}\right)$. By Definition 4.3.2 and Lemma 4.3.3, there must exist power allocation policies $\mathbf{P}^{(1)}(\boldsymbol{\nu})$ and $\mathbf{P}^{(2)}(\boldsymbol{\nu})$ in the form expressed in Lemma 4.3.3 such that, for all $l=1,2, \bar{\varepsilon}_{s, i}^{(l)}=\sum_{j: i \in \mathscr{S}_{n}(j)} E\left[\zeta_{1}(\boldsymbol{\nu}, j)+\zeta_{2}(\boldsymbol{\nu}, j)\right]$, for $1 \leq i \leq M$ and $0 \leq j \leq 2^{M}-1$. Also, $1-\varepsilon_{p}^{(l)}=E\left[\sum_{i=0}^{2^{M}-1} \psi_{1}^{(l)}(\boldsymbol{\nu}, j)\right]$ and $\overline{\mathbf{P}}^{(l)}=E\left[\mathbf{P}^{(l)}(\boldsymbol{\nu})\right]=$ $E\left[\sum_{j=0}^{2^{M}-1} \sum_{k=1}^{2} \zeta_{k}(\boldsymbol{\nu}, j) \mathbf{p}_{k}(\boldsymbol{\nu}, j)\right]$. To prove convexity of $\mathcal{Q}_{I}\left(\mathbf{r}_{o}\right)$, we must show that $\left(\delta \overline{\boldsymbol{\varepsilon}}_{s}^{(1)}+(1-\delta) \overline{\boldsymbol{\varepsilon}}_{s}^{(2)}, \delta \varepsilon_{p}^{(1)}+(1-\delta) \varepsilon_{p}^{(2)}, \delta \overline{\mathbf{P}}^{(1)}+(1-\delta) \overline{\mathbf{P}}^{(2)}\right) \in \mathcal{Q}_{I}\left(\mathbf{r}_{o}\right)$, where $\delta \in[0,1]$. Again, we define another random power policy $\mathbf{P}^{(x)}(\boldsymbol{\nu})$ that is randomized using the power policy $\mathbf{P}^{(1)}(\boldsymbol{\nu})$ with probability $\delta$ and the policy $\mathbf{P}^{(2)}(\boldsymbol{\nu})$ with probability $1-\delta$. By repeating a similar procedure to Appendix C.6, eventually, we can show that $\mathcal{Q}_{I}\left(\mathbf{r}_{o}\right)$ is a convex set. Finally, the convexity of $\mathcal{Q}_{I}\left(\mathbf{r}_{o}\right)$ also implies that the individual usage probability region $\overline{\mathcal{O}}_{I}\left(\mathbf{P}_{a v}, \epsilon_{p}, \mathbf{r}_{o}\right)$ is convex.

\section{C.11 KKT conditions corresponding to Problem} $(4.56)$

From the associated (necessary and sufficient) KKT conditions, we have

$$
\begin{gathered}
\frac{\partial l(\ldots)}{\partial p_{k, i}^{*}(\boldsymbol{\nu}, j)}\left\{\begin{array}{cc}
=0, & p_{k, i}^{*}(\boldsymbol{\nu}, j)>0 \\
\leq 0, & p_{k, i}^{*}(\boldsymbol{\nu}, j)=0
\end{array}, \quad \forall j, k\right. \\
\frac{\partial l(\ldots)}{\partial \zeta_{k}^{*}(\boldsymbol{\nu}, j)}\left\{\begin{array}{ll}
=0, & \zeta_{k}^{*}(\boldsymbol{\nu}, j) \geq 0 \\
\leq 0, & \zeta_{k}^{*}(\boldsymbol{\nu}, j)=0
\end{array}, \quad \forall j, k\right. \\
\lambda^{* T}\left(\mathbf{P}_{a v}-E\left[\sum_{j=0}^{2^{M}-1} \sum_{k=1}^{2} \zeta_{k}^{*}(\boldsymbol{\nu}, j) \mathbf{p}_{k}^{*}(\boldsymbol{\nu}, j)\right]\right)=0 \\
s^{*}\left(E\left[\sum_{j=0}^{2^{M}-1} \zeta_{1}^{*}(\boldsymbol{\nu}, j)\right]-\left(1-\epsilon_{p}\right)\right)=0 \\
\phi^{*}(\boldsymbol{\nu}, j)\left[\zeta_{1}^{*}(\boldsymbol{\nu}, j)\left(\mathcal{P}_{p}(g)-\boldsymbol{\beta}^{T} \mathbf{p}_{1}^{*}(\boldsymbol{\nu}, j)\right)\right]=0, \forall j, k
\end{gathered}
$$




$$
\begin{gathered}
\Gamma^{*}(\boldsymbol{\nu})\left[1-\sum_{j=0}^{2^{M}-1} \sum_{k=1}^{2} \zeta_{k}^{*}(\boldsymbol{\nu}, j)\right]=0, \forall j \\
\mathbf{p}_{k}^{*}(\boldsymbol{\nu}, j) \succeq \mathbf{0}, \forall j, k \\
\zeta_{k}^{*}(\boldsymbol{\nu}, j) \geq 0, \forall j, k \\
\boldsymbol{\xi}(j) \mathbf{r}_{o} \in \mathcal{C}_{M A C}\left(\boldsymbol{\nu}, \mathbf{p}_{k}^{*}(\boldsymbol{\nu}, j)\right), \forall j, k .
\end{gathered}
$$

\section{C.12 Proof of Proposition 4.3.4}

Note that $N_{j}\left(\sum_{i \in \mathscr{S}_{n}(j)} \mu_{i}\right), \Gamma^{*}(\boldsymbol{\nu}), s^{*}$ and $\phi^{*}(\boldsymbol{\nu}, j) P_{p}(g)$ are all independent of $\mathbf{p}_{k}(\boldsymbol{\nu}, j)$. Thus, the subproblems can be reformulated as

$$
\begin{array}{ll}
\text { Subproblem for } \mathbf{p}_{2}(\boldsymbol{\nu}, j): & \min _{\mathbf{p}_{2}(\boldsymbol{\nu}, j)} \lambda^{* T} \mathbf{p}_{2}(\boldsymbol{\nu}, j) \\
& \text { s.t. } \boldsymbol{\xi}(j) \mathbf{r}_{o} \in \mathcal{C}_{M A C}\left(\boldsymbol{\nu}, \mathbf{p}_{2}(\boldsymbol{\nu}, j)\right) .
\end{array}
$$

Subproblem for $\mathbf{p}_{1}(\boldsymbol{\nu}, j): \min _{\mathbf{p}_{1}(\boldsymbol{\nu}, j)} \boldsymbol{\eta}(j)^{* T} \mathbf{p}_{1}(\boldsymbol{\nu}, j)$

$$
\begin{array}{ll}
\text { s.t. } & \xi(j) \mathbf{r}_{o} \in \mathcal{C}_{M A C}\left(\boldsymbol{\nu}, \mathbf{p}_{1}(\boldsymbol{\nu}, j)\right), \\
& \phi^{*}(\boldsymbol{\nu}, j)\left[P_{p}(g)-\boldsymbol{\beta}^{T} \mathbf{p}_{1}(\boldsymbol{\nu}, j)\right]=0 .
\end{array}
$$

For a given rate vector $\mathbf{r}_{o}$ and channel state $\boldsymbol{\nu}$, we define the set of received powers that can support $\mathbf{r}_{o}, \tilde{\mathscr{G}}\left(\boldsymbol{\xi}(j) \mathbf{r}_{o}^{*}\right)$, as follows:

$$
\tilde{\mathscr{G}}\left(\boldsymbol{\xi}(j) \mathbf{r}_{o}^{*}\right)=\left\{\mathbf{q}: q_{i}=\bar{h}_{i} p_{i}, \boldsymbol{\xi}(j) \mathbf{r}_{o} \in \mathcal{C}_{M A C}(\boldsymbol{\nu}, \mathbf{p})\right\}
$$

which is a contra-polymatroid with rank function: $f(S)=\exp \left(\xi(j) \mathbf{r}_{o}(S)\right)-1$.

Then, we can apply Lemma 4.3.2 to solve for $\mathbf{p}_{1}^{*}(\boldsymbol{\nu}, j)$ and $\mathbf{p}_{2}^{*}(\boldsymbol{\nu}, j)$ in a similar manner as previously shown Appendix C.8. 


\section{Appendix D}

\section{Proofs in Chapter 5}

\section{D.1 Proof of Lemma 5.2.1}

Proof. The proof of CPS is based on the nearest neighbourhood condition as a given $\hat{\nu}$ corresponds to only one power level $p_{j}$, i.e.

$$
\begin{aligned}
\mathcal{R}_{j}=\{(x, \Gamma) \mid & {\left[1\left(p_{j}<\frac{c_{S U}}{\Gamma}\right)+\lambda p_{j}+s 1\left(p_{j}>x\right)\right] } \\
< & {\left.\left[1\left(p_{i}<\frac{c_{S U}}{\Gamma}\right)+\lambda p_{i}+s 1\left(p_{i}>x\right)\right], \forall i \neq j\right\} }
\end{aligned}
$$

For simplicity, define $l\left(p_{j}\right)=1\left(p_{i}<\frac{c_{S U}}{\Gamma}\right)+\lambda p_{i}+s 1\left(p_{i}>x\right)$.

For given $\lambda, s$ and power codebook $\mathbf{P}$, if SU allows an outage, it uses the lowest power level $p_{L}$. Otherwise, it can use the lowest power level $p_{j, \min }$ such that $p_{j, \min } \geq$ $\frac{c_{S U}}{\Gamma}$. For $x<p_{L}$, the $\mathrm{PU}$ will face an outage regardless of the power level that SU uses. Also, for $x \geq p_{j, \min }$, using power level $p_{j, \text { min }}$ does not disturb PU as the interference cannot pull PU to be in outage. Therefore in these two cases, SU transmits with $p_{j}$ when $\Gamma \in\left[\frac{c_{S U}}{p_{j}}, \frac{c_{S U}}{p_{j+1}}\right)$ for $j \neq L$ while using $p_{L}$ when $\Gamma \in$ $\left[0, \frac{c_{S U}}{p_{1}}\right) \cup\left[\frac{c_{S U}}{p_{L}}, \infty\right]$

It is important to note that $p_{1} \leq p_{L}+\frac{1}{\lambda}$, which can be proved by a contradiction. First, assume that $p_{1}>p_{L}+\frac{1}{\lambda}$. Then, for a given channel state that $\Gamma \in\left(\frac{c_{S U}}{p_{1}}, \frac{c_{S U}}{p_{2}}\right]$ and $x<p_{L}$. Thus, the Lagrangian from $p_{1}$ (non-outage case) becomes $l\left(p_{1}\right)=\lambda p_{1}>$ $l\left(p_{L}\right)=1+\lambda p_{L}$, implying that even though a feedback resource is dedicated to $p_{1}$, it would never be used.

The more complicated case is when $p_{L} \leq x<p_{j, \min }$ and $s>0$, since the SU can either make PU in outage but meet SU's target rate or use $p_{L}$ to protect PU but allows outage at SU. To determine this, SU compares the Lagrangian regarding to 
$p_{j}$ and $p_{L}$. SU will transmit with $p_{j}$ to support its target rate if

$$
\begin{aligned}
l\left(p_{L}\right) & >l\left(p_{j}\right) \\
1+\lambda p_{L} & >\lambda p_{j, \min }+s \\
p_{L}+\frac{1-s}{\lambda} & >p_{j, \text { min }}
\end{aligned}
$$

Otherwise, $\mathrm{SU}$ will allow an outage if $p_{j, \min }>p_{L}+\frac{1-s}{\lambda}$, i.e. $\mathrm{SU}$ tends to save power and protect $\mathrm{PU}$ links rather than trying to meet target rate with very high power $p_{j, \text { min }}$. For simplicity, define $k=\arg \min _{j: p_{j}>p_{L}+\frac{1-s}{\lambda}} p_{j}$. This implies that there is no need for the SU to check the condition in (D.2) for $k+1 \leq j \leq L$.

\section{D.2 All KKT conditions for a locally optimal so- lution}

For $j=1$,

$$
\begin{aligned}
0 & =-\frac{c_{S U}}{p_{1}^{2}} F_{\Gamma}^{\prime}\left(\frac{c_{S U}}{p_{1}}\right)+\tau_{G}\left(\frac{c_{S U}}{p_{1}^{2}} F_{\Gamma}^{\prime}\left(\frac{c_{S U}}{p_{1}}\right)\left[F_{\tilde{X}}\left(p_{1}\right)-F_{\tilde{X}}\left(p_{2}\right)\right]+F_{\tilde{X}}^{\prime}\left(p_{1}\right)\left[F_{\Gamma}\left(\frac{c_{S U}}{p_{2}}\right)-F_{\Gamma}\left(\frac{c_{S U}}{p_{1}}\right)\right]\right) \\
& +\lambda\left\{\left[F_{\Gamma}\left(\frac{c_{S U}}{p_{2}}\right)-F_{\Gamma}\left(\frac{c_{S U}}{p_{1}}\right)\right]-\frac{c_{S U}}{p_{1}^{2}} p_{L} F_{\Gamma}^{\prime}\left(\frac{c_{S U}}{p_{1}}\right)+\tau_{G}\left(p_{1}-p_{2}\right)\left[F_{\tilde{X}}\left(p_{1}\right)-F_{\tilde{X}}\left(p_{L}\right)\right] \frac{c_{S U}}{p_{1}^{2}} F_{\Gamma}^{\prime}\left(\frac{c_{S U}}{p_{1}}\right)\right\} \\
& -\lambda \tau_{G}\left\{\left(\left[F_{\Gamma}\left(\frac{c_{S U}}{p_{2}}\right)-F_{\Gamma}\left(\frac{c_{S U}}{p_{1}}\right)\right]\left[F_{\tilde{X}}\left(p_{1}\right)-F_{\tilde{X}}\left(p_{2}\right)\right]+\left(p_{1}-p_{2}\right)\left[F_{\Gamma}\left(\frac{c_{S U}}{p_{2}}\right)-F_{\Gamma}\left(\frac{c_{S U}}{p_{1}}\right)\right] F_{\tilde{X}}^{\prime}\left(p_{1}\right)\right)\right\}
\end{aligned}
$$

For $1<j<k+1$,

$$
\begin{gathered}
0=\tau_{G}\left[\left[F_{\tilde{X}}\left(p_{j}\right)-F_{\tilde{X}}\left(p_{j-1}\right)\right] \frac{c_{S U}}{p_{j}^{2}} F_{\Gamma}^{\prime}\left(\frac{c_{S U}}{p_{j}}\right)+\left(F_{\Gamma}\left(\frac{c_{S U}}{p_{j+1}}\right)-F_{\Gamma}\left(\frac{c_{S U}}{p_{j}}\right)\right) F_{\tilde{X}}^{\prime}\left(p_{j}\right)\right] \\
+\lambda\left\{\left[F_{\Gamma}\left(\frac{c_{S U}}{p_{j+1}}\right)-F_{\Gamma}\left(\frac{c_{S U}}{p_{j}}\right)\right]+\left(p_{j}-p_{j-1}\right) \frac{c_{S U}}{p_{j}^{2}} F_{\Gamma}^{\prime}\left(\frac{c_{S U}}{p_{j}}\right)\right. \\
-\tau_{G}\left(\left[F_{\Gamma}\left(\frac{c_{S U}}{p_{j+1}}\right)-F_{\Gamma}\left(\frac{c_{S U}}{p_{j}}\right)\right]\left(F_{\tilde{X}}\left(p_{j}\right)-F_{\tilde{X}}\left(p_{L}\right)\right)\right. \\
+\left(p_{j}-p_{L}\right)\left[F_{\Gamma}\left(\frac{c_{S U}}{p_{j+1}}\right)-F_{\Gamma}\left(\frac{c_{S U}}{p_{j}}\right)\right] F_{\tilde{X}}^{\prime}\left(p_{j}\right) \\
\left.\left.-\left(p_{j}-p_{L}\right)\left[F_{\tilde{X}}\left(p_{j}\right)-F_{\tilde{X}}\left(p_{L}\right)\right] \frac{c_{S U}}{p_{j}^{2}} F_{\Gamma}^{\prime}\left(\frac{c_{S U}}{p_{j}}\right)\right)\right\}
\end{gathered}
$$


For $j=k+1$,

$$
\begin{aligned}
& 0=-\tau_{G}\left(F_{\tilde{X}}\left(p_{k}\right)-F_{\tilde{X}}\left(p_{L}\right)\right) \frac{c_{S U}}{p_{k+1}^{2}} F_{\Gamma}^{\prime}\left(\frac{c_{S U}}{p_{k+1}}\right) \\
&+s \tau_{G}\left\{\left[F_{\Gamma}\left(\frac{c_{S U}}{p_{k+2}}\right)-F_{\Gamma}\left(\frac{c_{S U}}{p_{k+1}}\right)\right] F_{\tilde{X}}^{\prime}\left(p_{k+1}\right)+\left[F_{\tilde{X}}\left(p_{k+1}\right)-F_{\tilde{X}}\left(p_{L}\right)\right] \frac{c_{S U}}{p_{k+1}^{2}} F_{\Gamma}^{\prime}\left(\frac{c_{S U}}{p_{k+1}}\right)\right\} \\
&+\lambda\left\{\left[F_{\Gamma}\left(\frac{c_{S U}}{p_{k+2}}\right)-F_{\Gamma}\left(\frac{c_{S U}}{p_{k+1}}\right)\right]+\left[p_{k+1}-p_{k}\right] \frac{c_{S U}}{p_{k+1}^{2}} F_{\Gamma}^{\prime}\left(\frac{c_{S U}}{p_{k+1}}\right)\right. \\
&\left.\quad+\tau_{G}\left(p_{k}-p_{L}\right)\left[F_{\tilde{X}}\left(p_{k+1}\right)-F_{\tilde{X}}\left(p_{L}\right)\right] \frac{c_{S U}}{p_{k+1}^{2}} F_{\Gamma}^{\prime}\left(\frac{c_{S U}}{p_{k+1}}\right)\right\}
\end{aligned}
$$

For $k+1<j<L$,

$$
\begin{aligned}
0= & +s \tau_{G}\left[\left(F_{\Gamma}\left(\frac{c_{S U}}{p_{j+1}}\right)-F_{\Gamma}\left(\frac{c_{S U}}{p_{j}}\right)\right) F_{\tilde{X}}^{\prime}\left(p_{j}\right)+\left[F_{\tilde{X}}\left(p_{j}\right)-F_{\tilde{X}}\left(p_{j-1}\right)\right] \frac{c_{S U}}{p_{j}^{2}} F_{\Gamma}^{\prime}\left(\frac{c_{S U}}{p_{j}}\right)\right] \\
& +\lambda\left[\left(F_{\Gamma}\left(\frac{c_{S U}}{p_{j+1}}\right)-F_{\Gamma}\left(\frac{c_{S U}}{p_{j}}\right)\right)+\frac{c_{S U}}{p_{j}^{2}} F_{\Gamma}^{\prime}\left(\frac{c_{S U}}{p_{j}}\right)\left[p_{j}-p_{j-1}\right]\right]
\end{aligned}
$$

For $j=L$,

$$
\begin{aligned}
& 0=-\tau_{G} F_{\tilde{X}}^{\prime}\left(p_{L}\right)\left(\sum_{j=1}^{k}\left[F_{\Gamma}\left(\frac{c_{S U}}{p_{j+1}}\right)-F_{\Gamma}\left(\frac{c_{S U}}{p_{j}}\right)\right]\right) \\
& +s \tau_{G}\left\{F_{\tilde{X}}^{\prime}\left(p_{L}\right)-\right. \\
& \left.\quad \sum_{j=k+1}^{L-1}\left[F_{\Gamma}\left(\frac{c_{S U}}{p_{j+1}}\right)-F_{\Gamma}\left(\frac{c_{S U}}{p_{j}}\right)\right] F_{\tilde{X}}^{\prime}\left(p_{L}\right)-\frac{c_{S U}}{p_{L}^{2}} F_{\Gamma}^{\prime}\left(\frac{c_{S U}}{p_{j}}\right)\left[F_{\tilde{X}}\left(p_{L-1}\right)-F_{\tilde{X}}\left(p_{L}\right)\right]\right\} \\
& +\lambda\left\{\left[1-F_{\Gamma}\left(\frac{c_{S U}}{p_{1}}\right)-F_{\Gamma}\left(\frac{c_{S U}}{p_{L}}\right)\right]+\left(p_{L}-p_{L-1}\right) \frac{c_{S U}}{p_{L}^{2}} F_{\Gamma}^{\prime}\left(\frac{c_{S U}}{p_{j}}\right)\right. \\
& +\tau_{G} \sum_{j=1}^{k}\left(\left[F_{\Gamma}\left(\frac{c_{S U}}{p_{j+1}}\right)-F_{\Gamma}\left(\frac{c_{S U}}{p_{j}}\right)\right]\left[F_{\tilde{X}}\left(p_{j}\right)-F_{\tilde{X}}\left(p_{L}\right)\right]\right. \\
& \left.\left.\quad+\left(p_{j}-p_{L}\right)\left[F_{\Gamma}\left(\frac{c_{S U}}{p_{j+1}}\right)-F_{\Gamma}\left(\frac{c_{S U}}{p_{j}}\right)\right] F_{\tilde{X}}^{\prime}\left(p_{j}\right)\right)\right\}
\end{aligned}
$$

\section{D.3 Proof of Lemma 5.2.2}

We adopt a similar proof technique to that used in [61]. 
Proof. First, (D.7) can be rewritten as

$$
\begin{aligned}
& \frac{c_{S U}}{p_{L}^{2}} F_{\Gamma}^{\prime}\left(\frac{c_{S U}}{p_{L}}\right)\left[s \tau_{G}\left[F_{\tilde{X}}\left(p_{L-1}\right)-F_{\tilde{X}}\left(p_{L}\right)\right]+\lambda\left(p_{L-1}-p_{L}\right)\right] \\
& =F_{\tilde{X}}^{\prime}\left(p_{L}\right) \tau_{G}\left[s\left(1-F_{\Gamma}\left(\frac{c_{S U}}{p_{L}}\right)+F_{\Gamma}\left(\frac{c_{S U}}{p_{1}}\right)\right)+\sum_{j=1}^{k}\left[\lambda\left(p_{j}-p_{L}\right)-(1-s)\right]\left(F_{\Gamma}\left(\frac{c_{S U}}{p_{j+1}}\right)-F_{\Gamma}\left(\frac{c_{S U}}{p_{j}}\right)\right)\right] \\
& \quad+\lambda\left[1-F_{\Gamma}\left(\frac{c_{S U}}{p_{L}}\right)+\tilde{G}_{O}\right] \\
& \geq \lambda\left[1-F_{\Gamma}\left(\frac{c_{S U}}{p_{L}}\right)+\tilde{G}_{O}\right]
\end{aligned}
$$

where $\tilde{G}_{O}$ is the value of $G_{O}$ at the optimum. Note that the inequality follows from the fact that the positive term is discarded.

Now taking the limit as $L \rightarrow \infty$ and assume that $\lim _{L \rightarrow \infty} p_{L}=\delta_{L}>0$. Then, we have that

$$
\begin{aligned}
& \lim _{L \rightarrow \infty} \frac{c_{S U}}{p_{L}^{2}} F_{\Gamma}^{\prime}\left(\frac{c_{S U}}{p_{L}}\right)\left[s \tau_{G}\left[F_{\tilde{X}}\left(p_{L-1}\right)-F_{\tilde{X}}\left(p_{L}\right)\right]+\lambda\left(p_{L-1}-p_{L}\right)\right] \\
& \geq \lim _{L \rightarrow \infty} \lambda\left[1-F_{\Gamma}\left(\frac{c_{S U}}{p_{L}}\right)+\tilde{G}_{O}\right] \\
& \geq \lambda\left[1-F_{\Gamma}\left(\frac{c_{S U}}{p_{L}}\right)+\tilde{G}_{O}^{F-C S I T}\right] \\
& >0
\end{aligned}
$$

where $\tilde{G}_{O}^{F-C S I T}$ is the value of the objective function at the optimum when SU has perfect CSI.

As $p_{1}>p_{2}>\ldots>p_{L}>0$, the sequence $\left\{p_{j}\right\}_{j=1}^{L}$ is a monotonically decreasing sequence and bounded below by $\delta_{L}$, thereby converging to its greatest lower bound $\delta_{L}$ as $L \rightarrow \infty$. Hence, $\left(p_{L-1}-p_{L}\right) \rightarrow 0$ as $L \rightarrow \infty$, implying that

$$
\begin{aligned}
& \lim _{L \rightarrow \infty} \frac{c_{S U}}{p_{L}^{2}} F_{\Gamma}^{\prime}\left(\frac{c_{S U}}{p_{L}}\right)\left[s \tau_{G}\left[F_{\tilde{X}}\left(p_{L-1}\right)-F_{\tilde{X}}\left(p_{L}\right)\right]+\lambda\left(p_{L-1}-p_{L}\right)\right] \\
& =\frac{c_{S U}}{\delta_{L}^{2}} F_{\Gamma}^{\prime}\left(\frac{c_{S U}}{\delta_{L}}\right)\left[s \tau_{G}\left[F_{\tilde{X}}\left(p_{L-1}\right)-F_{\tilde{X}}\left(p_{L}\right)\right]+\lambda\left(p_{L-1}-p_{L}\right)\right] \\
& =0
\end{aligned}
$$

Obviously, the result in (D.10) contradicts (D.9) and the prior assumption that $\lim _{L \rightarrow \infty} p_{L}=\delta_{L}>0$. 


\section{D.4 GLA+SFA}

This algorithm is based on the generalized Lloyd's algorithm together with using a sigmoid function for indicator functions approximation, first proposed in [70]. More specifically, this algorithm again starts with a random power codebook to employ the nearest neighbourhood condition in Lloyd's algorithm as explained earlier. Next, the power codebook is updated by $p_{j} \approx \min _{p_{j} \geq 0} E\left[\sigma\left(K\left(p_{j}-\frac{c_{S U}}{\Gamma}\right)\right)+\lambda p_{j}+s \sigma\left(K\left(p_{j}+x\right)\right) \mid \mathcal{R}_{j}\right]$ for $j=1, \ldots, L$ where $\sigma(w)=\frac{1}{1+\exp (w)}$ is the sigmoid function and the coefficient $K$ controls the steepness of the approximation. ( [70] provides more details on how to select the value of $K)$.

Note that, in any given region $\mathcal{R}_{j}$, searching for $p_{j}$ is not completely exhaustive. For $j<L$, all channel state $\nu \in \mathcal{R}_{j}$ must result in non-outage, so the range of $p_{j}$ lies between $\frac{c_{S U}}{\Gamma_{\max , j}}$ and $\frac{c_{S U}}{\Gamma_{\min , j}}$, where $\Gamma_{\max , j}=\max _{\nu \in \mathcal{R}_{j}} \Gamma(\nu)$ and $\Gamma_{\min , j}=\min _{\nu \in \mathcal{R}_{j}} \Gamma(\nu)$. For $j=L, \mathcal{R}_{j}$ consists of the non-outage region $\left(\mathcal{R}_{L}^{n}\right)$ and the outage region $\left(\mathcal{R}_{L}^{o}\right)$. Thus the updated power codebook must lie in $\left[0, \frac{c_{S U}}{\min _{\nu \in \mathcal{R}_{L}^{n}} \Gamma}\right]$. Note that the range starts from zero power for the case that SU will transmit with the lowest power level when it allows outage. After the power codebook is updated, the algorithm repeats these two steps until convergence.

\section{D.5 All KKT conditions for ZFLP}

For $j=1$,

$$
\begin{aligned}
0= & -\frac{c_{S U}}{p_{1}^{2}}\left(1-\tau_{G} F_{\tilde{X}}\left(p_{1}\right)\right) F_{\Gamma}^{\prime}\left(\frac{c_{S U}}{p_{1}}\right)+\tau_{G}\left(F_{\Gamma}\left(\frac{c_{S U}}{p_{2}}\right)-F_{\Gamma}\left(\frac{c_{S U}}{p_{1}}\right)\right) F_{\tilde{X}}^{\prime}\left(p_{1}\right) \\
+\lambda & \left(F_{\Gamma}\left(\frac{c_{S U}}{p_{2}}\right)-F_{\Gamma}\left(\frac{c_{S U}}{p_{1}}\right)\right)\left[1-\tau_{G} F_{\tilde{X}}\left(p_{1}\right)-p_{1} \tau_{G} F_{\tilde{X}}^{\prime}\left(p_{1}\right)\right] \\
& \left.+p_{1}\left(1-\tau_{G} F_{\tilde{X}}\left(p_{1}\right)\right) \cdot \frac{c_{S U}}{p_{1}^{2}} F_{\Gamma}^{\prime}\left(\frac{c_{S U}}{p_{1}}\right)\right]
\end{aligned}
$$

For $1<j<k+1$,

$$
\begin{aligned}
0= & \tau_{G}\left[\left(F_{\Gamma}\left(\frac{c_{S U}}{p_{j+1}}\right)-F_{\Gamma}\left(\frac{c_{S U}}{p_{j}}\right)\right) F_{\tilde{X}}^{\prime}\left(p_{j}\right)+\frac{c_{S U}}{p_{j}^{2}} F_{\Gamma}^{\prime}\left(\frac{c_{S U}}{p_{j}}\right)\left[F_{\tilde{X}}\left(p_{j}\right)-F_{\tilde{X}}\left(p_{j-1}\right)\right]\right] \\
+\lambda & {\left[\left(F_{\Gamma}\left(\frac{c_{S U}}{p_{j+1}}\right)-F_{\Gamma}\left(\frac{c_{S U}}{p_{j}}\right)\right)\left[1-\tau_{G} F_{\tilde{X}}\left(p_{j}\right)-p_{j} \tau_{G} F_{\tilde{X}}^{\prime}\left(p_{j}\right)\right]\right.} \\
& \left.+\left[p_{j}\left(1-\tau_{G} F_{\tilde{X}}\left(p_{j}\right)\right)-p_{j-1}\left(1-\tau_{G} F_{\tilde{X}}\left(p_{j-1}\right)\right)\right] \cdot \frac{c_{S U}}{p_{j}^{2}} F_{\Gamma}^{\prime}\left(\frac{c_{S U}}{p_{j}}\right)\right]
\end{aligned}
$$


For $j=k+1$,

$$
\begin{aligned}
& 0=-e^{-g_{T}} F_{\tilde{X}}\left(p_{k}\right) \frac{c_{S U}}{p_{k+1}^{2}} F_{\Gamma}^{\prime}\left(\frac{c_{S U}}{p_{k+1}}\right) \\
&+ s\left(\tau_{G}\left[\left(F_{\Gamma}\left(\frac{c_{S U}}{p_{k+2}}\right)-F_{\Gamma}\left(\frac{c_{S U}}{p_{k+1}}\right)\right) F_{\tilde{X}}^{\prime}\left(p_{k+1}\right)+\frac{c_{S U}}{p_{k+1}^{2}} F_{\Gamma}^{\prime}\left(\frac{c_{S U}}{p_{k+1}}\right) F_{\tilde{X}}\left(p_{k+1}\right)\right]\right) \\
&+\lambda {\left[\left(F_{\Gamma}\left(\frac{c_{S U}}{p_{k+2}}\right)-F_{\Gamma}\left(\frac{c_{S U}}{p_{k+1}}\right)\right) F_{\tilde{X}}^{\prime}\left(p_{k+1}\right)+\frac{c_{S U}}{p_{k+1}^{2}} F_{\Gamma}^{\prime}\left(\frac{c_{S U}}{p_{k+1}}\right)\left[p_{k+1}-p_{k}\right]\right.} \\
&\left.\quad+\frac{c_{S U}}{p_{k+1}^{2}} F_{\Gamma}^{\prime}\left(\frac{c_{S U}}{p_{k+1}}\right)\left[p_{k}\left(1-\tau_{G} F_{\tilde{X}}\left(p_{k}\right)\right)\right]\right] \\
&=-e^{-g_{T}} F_{\tilde{X}}\left(p_{k}\right) \frac{c_{S U}}{p_{k+1}^{2}} F_{\Gamma}^{\prime}\left(\frac{c_{S U}}{p_{k+1}}\right) \\
&+s\left(\tau_{G}\left[\left(F_{\Gamma}\left(\frac{c_{S U}}{p_{k+2}}\right)-F_{\Gamma}\left(\frac{c_{S U}}{p_{k+1}}\right)\right) F_{\tilde{X}}^{\prime}\left(p_{k+1}\right)+\frac{c_{S U}}{p_{k+1}^{2}} F_{\Gamma}^{\prime}\left(\frac{c_{S U}}{p_{k+1}}\right) F_{\tilde{X}}\left(p_{k+1}\right)\right]\right) \\
&+\lambda\left[\left(F_{\Gamma}\left(\frac{c_{S U}}{p_{k+2}}\right)-F_{\Gamma}\left(\frac{c_{S U}}{p_{k+1}}\right)\right) F_{\tilde{X}}^{\prime}\left(p_{k+1}\right)+\frac{c_{S U}}{p_{k+1}^{2}} F_{\Gamma}^{\prime}\left(\frac{c_{S U}}{p_{k+1}}\right)\left[p_{k+1}-p_{k} \tau_{G} F_{\tilde{X}}\left(p_{k}\right)\right]\right]
\end{aligned}
$$

For $k+1<j<L-1$,

$$
\begin{aligned}
0 & =0+s\left(\tau_{G}\left[\left(F_{\Gamma}\left(\frac{c_{S U}}{p_{j+1}}\right)-F_{\Gamma}\left(\frac{c_{S U}}{p_{j}}\right)\right) F_{\tilde{X}}^{\prime}\left(p_{j}\right)+\frac{c_{S U}}{p_{j}^{2}} F_{\Gamma}^{\prime}\left(\frac{c_{S U}}{p_{j}}\right)\left[F_{\tilde{X}}\left(p_{j}\right)-F_{\tilde{X}}\left(p_{j-1}\right)\right]\right]\right) \\
& +\lambda\left[\left(F_{\Gamma}\left(\frac{c_{S U}}{p_{j+1}}\right)-F_{\Gamma}\left(\frac{c_{S U}}{p_{j}}\right)\right)+\frac{c_{S U}}{p_{j}^{2}} F_{\Gamma}^{\prime}\left(\frac{c_{S U}}{p_{j}}\right)\left[p_{j}-p_{j-1}\right]\right]
\end{aligned}
$$

For $j=L-1$,

$$
\begin{aligned}
0 & =0+s\left(\tau_{G}\left[\left(1-F_{\Gamma}\left(\frac{c_{S U}}{p_{L-1}}\right)\right) F_{\tilde{X}}^{\prime}\left(p_{L-1}\right)+\frac{c_{S U}}{p_{L-1}^{2}} F_{\Gamma}^{\prime}\left(\frac{c_{S U}}{p_{L-1}}\right)\left[F_{\tilde{X}}\left(p_{L-1}\right)-F_{\tilde{X}}\left(p_{L-2}\right)\right]\right]\right) \\
& +\lambda\left[\left(1-F_{\Gamma}\left(\frac{c_{S U}}{p_{L-1}}\right)\right)+\frac{c_{S U}}{p_{L-1}^{2}} F_{\Gamma}^{\prime}\left(\frac{c_{S U}}{p_{L-1}}\right)\left[p_{L-1}-p_{L-2}\right]\right]
\end{aligned}
$$

\section{D.6 Proof of Lemma 5.3.1}

Proof. For $1<j<k+1$, we first simplify (D.4) as follows

$$
\begin{aligned}
0 & =\left\{\lambda+\left(\lambda\left(F_{\tilde{X}}\left(p_{L}\right)-F_{\tilde{X}}\left(p_{j}\right)\right)+F_{\tilde{X}}^{\prime}\left(p_{j}\right)\left(1-\lambda\left(p_{j}-p_{L}\right)\right)\right) \tau_{G}\right\}\left(\frac{p_{j}}{p_{j+1}}-\frac{p_{j-1}}{p_{j}}\right) \\
& =\left(\frac{p_{j}}{p_{j+1}}-\frac{p_{j-1}}{p_{j}}\right)
\end{aligned}
$$

For $k+1<j<L$, (D.6) can be simplified as follows

$$
\begin{aligned}
0 & =s \tau_{G}\left[F_{\tilde{X}}^{\prime}\left(p_{j}\right)\left(\frac{1}{p_{j+1}}-\frac{1}{p_{j}}\right)+\left[F_{\tilde{X}}\left(p_{j}\right)-F_{\tilde{X}}\left(p_{j-1}\right)\right] \frac{1}{p_{j}^{2}}\right]+\lambda\left[\frac{1}{p_{j+1}}-\frac{p_{j-1}}{p_{j}^{2}}\right] \\
& =\left(\frac{p_{j}}{p_{j+1}}-\frac{p_{j-1}}{p_{j}}\right)
\end{aligned}
$$




\section{D.7 Proof of Lemma 5.3.2}

For $1 \leq j \leq k$,

$$
p_{j} f_{1}^{\prime}\left(p_{j}\right)\left[F_{\Gamma}\left(v_{j+1}\right)-F_{\Gamma}\left(v_{j}\right)\right]=p_{j-1}\left[\frac{f_{1}\left(p_{j-1}\right)-f_{1}\left(p_{j}\right)}{p_{j-1}-p_{j}}\right]\left(v_{j}-v_{j-1}\right) F_{\Gamma}^{\prime}\left(v_{j}\right)
$$

As $L \rightarrow \infty$, it yields $p_{j} f_{1}^{\prime}\left(p_{j}\right) F_{\Gamma}^{\prime}\left(v_{j}\right)\left[v_{j+1}-v_{j}\right] \approx p_{j-1} f_{1}^{\prime}\left(p_{j-1}\right)\left(v_{j}-v_{j-1}\right) F_{\Gamma}^{\prime}\left(v_{j}\right)$. Thus, we have $\frac{p_{j}}{p_{j+1}} \approx \frac{p_{j-1}}{p_{j}}=\theta=1+\epsilon_{\theta}$, where $\theta$ can be approximated from solving

$$
\left[\frac{1-f_{1}\left(p_{1}\right)+p_{1} f_{1}^{\prime}\left(p_{1}\right)}{p_{1} f_{1}^{\prime}\left(p_{1}\right)}\right] \approx \frac{v_{2}}{v_{1}}=\theta=\left[\frac{1-f_{1}\left(\frac{p_{0}}{\theta}\right)+\frac{p_{0}}{\theta} f_{1}^{\prime}\left(\frac{p_{0}}{\theta}\right)}{\frac{p_{0}}{\theta} f_{1}^{\prime}\left(\frac{p_{0}}{\theta}\right)}\right]
$$

where $p_{0} \triangleq \frac{1}{\lambda}$. Note that $f_{1}\left(p_{0}\right)=1, v_{j}=p_{j-1}\left(\frac{v_{j}-v_{j-1}}{p_{j-1}-p_{j}}\right)$.

\section{D.8 Proof of Lemma 5.3.3}

For $j=k+1$,

$$
p_{k+1} f_{2}^{\prime}\left(p_{k+1}\right)\left[F_{\Gamma}\left(v_{k+2}\right)-F_{\Gamma}\left(v_{k+1}\right)\right]=p_{k}\left[\frac{f_{1}\left(p_{k}\right)-f_{2}\left(p_{k+1}\right)}{p_{k}-p_{k+1}}\right]\left(v_{k+1}-v_{k}\right) F_{\Gamma}^{\prime}\left(v_{k+1}\right)
$$

As $L \rightarrow \infty, \frac{v_{k+2}}{v_{k+1}}=\frac{p_{k+1}}{p_{k+2}} \approx\left[\frac{f_{1}\left(p_{k}\right)-f_{2}\left(\frac{p_{k}}{\kappa}\right)+\frac{p_{k}}{\kappa} f_{2}^{\prime}\left(\frac{p_{k}}{\kappa}\right)}{\frac{p_{k}}{\kappa} f_{1}^{\prime}\left(\frac{p_{k}}{\kappa}\right)}\right]=\kappa=1+\epsilon_{\kappa}$. Therefore,

$$
\begin{aligned}
\kappa & =\frac{\lambda p_{k}+\frac{c_{o}\left(1-\lambda p_{k}\right)}{1+c_{o} p_{k}} e^{-g_{T}} p_{k}-s e^{-g_{T}} \frac{\left(c_{o} p_{k+1}\right)^{2}}{\left(1+c_{o} p_{k+1}\right)^{2}}}{p_{k+1}\left[\lambda+s e^{-g_{T}} \frac{c_{o}}{\left(1+c_{o} p_{k+1}\right)^{2}}\right]} \\
& =\theta p_{k+1} \frac{\lambda+\frac{c_{o}\left(1-\theta \lambda p_{k+1}\right)}{1+\theta c_{o} p_{k+1}} e^{-g_{T}-s e^{-g_{T}} \frac{c_{o}^{2} p_{k+1}}{\left(1+c_{o} p_{k+1}\right)^{2}}}}{p_{k+1}\left[\lambda+s e^{-g_{T}} \frac{c_{o}}{\left(1+c_{o} p_{k+1}\right)^{2}}\right]} \\
& =\theta \cdot \frac{\lambda+e^{-g_{T}} \frac{c_{o}}{\left(1+c_{o} p_{k+1}\right)^{2}}\left[\frac{\left(1-\theta \lambda p_{k+1}\right)\left(1+c_{o} p_{k+1}\right)^{2}}{1+\theta c_{o} p_{k+1}}-s c_{o} p_{k+1}\right]}{\left[\lambda+s e^{-g_{T}} \frac{c_{o}}{\left(1+c_{o} p_{k+1}\right)^{2}}\right]} \\
& \stackrel{(b)}{\approx} \theta
\end{aligned}
$$

where $(b)$ is from the fact that $\lim _{L \rightarrow \infty} \theta=1+\lim _{L \rightarrow \infty} \epsilon_{\theta} \approx 1$ and $\lim _{L \rightarrow \infty} p_{k+1}=\frac{1-s}{\lambda}$, making $\lim _{L \rightarrow \infty}\left(1-\theta \lambda p_{k+1}\right) \approx s$ and $\lim _{L \rightarrow \infty} \frac{\left(1+c_{o} p_{k+1}\right)^{2}}{1+\theta c_{o} p_{k+1}} \approx 1+c_{o} p_{k+1}$. 


\section{D.9 Expressions of $\mathcal{F}_{P O C}(\theta, k)$ and $\mathcal{F}_{A T P C}(\theta, k)$}

For POC, (5.14) can be approximated by integration as follows.

$$
\begin{aligned}
& \epsilon_{p}-\left(1-e^{-g_{T}}\right) \\
& \approx e^{-g_{T}} \sum_{j=k+1}^{L-1} F_{\tilde{X}}\left(p_{j}\right) F_{\Gamma}^{\prime}\left(v_{j}\right)\left[v_{j+1}-v_{j}\right] \\
& =e^{-g_{T}} \sum_{j=k+1}^{L-1}\left[\frac{c_{o}}{1+c_{0} \frac{c_{S U} U}{v_{j}}}\right]\left(e^{\left.-N_{0} v_{j} \frac{N_{0}\left(1+P_{c} v_{j}\right)+P_{c}}{\left(1+P_{c} v_{j}\right)^{2}}\right) v_{j}[\theta-1]}\right. \\
& \approx e^{-g_{T}} c_{o} c_{S U}(\theta-1) \sum_{j=0}^{L-k-2} \frac{\theta^{j}}{\theta^{j}+\frac{c_{o}{ }^{c} S U}{v_{k+1}}}\left[\frac{N_{0}}{1+\theta^{j} P_{c} v_{k+1}}+\frac{P_{c}}{\left(1+\theta^{j} P_{c} v_{k+1}\right)^{2}}\right] \exp \left(-N_{0} v_{k+1} \theta^{j}\right) \\
& \approx e^{-g_{T}} c_{o} c_{S U}(\theta-1) \int_{z=0}^{L-k-1} \frac{\theta^{z}}{\theta^{z}+\frac{c_{0} c_{S U}}{v_{k+1}}}\left[\frac{N_{0}}{1+\theta^{z} P_{c} v_{k+1}}+\frac{P_{c}}{\left(1+\theta^{z} P_{c} v_{k+1}\right)^{2}}\right] \exp \left(-N_{0} v_{k+1} \theta^{z}\right)
\end{aligned}
$$

Let $t(y)=\frac{1}{y+\frac{c_{0} C_{S} U}{v_{k+1}}}\left[\frac{N_{0}}{1+y P_{c} v_{k+1}}+\frac{P_{c}}{\left(1+y P_{c} v_{k+1}\right)^{2}}\right] \exp \left(-N_{0} v_{k+1} y\right)$ and $s(z)=\theta^{z} t\left(\theta^{z}\right)$. By changing of variable $u=\theta^{z}$ and $d u=u \ln \theta d z$. Thus,

$$
\epsilon_{p}-\left(1-e^{-g_{T}}\right) \approx \ln \theta \int_{z=0}^{L-k-1} s(z) d z=A_{1}\left(v_{k+1}\right)-A_{1}\left(v_{k+1} \theta^{L-k-1}\right)=\mathcal{F}_{P O C}(\theta, k)
$$

where

$$
\begin{aligned}
A_{1}(z)= & \frac{N_{0}+\frac{P_{c}}{1-P_{c} c_{o} c_{S}}}{1-P_{c} c_{o} c_{S U}}\left[\exp \left(N_{0} c_{o} c_{S U}\right) E_{1}\left(N_{0}\left(c_{o} c_{S U}+z\right)\right)\right] \\
& -\frac{P_{c}}{1-P_{c} c_{o} c_{S U}}\left[\frac{\exp \left(-N_{0} z\right)}{1+P_{c} z}+\frac{\exp \left(\frac{N_{0}}{P_{c}}\right)}{1-P_{c} c_{o} c_{S U}} E_{1}\left(N_{0}\left[\frac{1}{P_{c}}+z\right]\right)\right]
\end{aligned}
$$

Note that as $L$ is large, $\lim _{L \rightarrow \infty} v_{k+1}=\frac{c_{S U} \lambda}{1-s}$.

Likewise, ATPC in (5.15) can be approximated as follows.

$$
\begin{aligned}
P_{a v} & \approx \sum_{j=k+1}^{L-1} p_{j} F_{\Gamma}^{\prime}\left(v_{j}\right)\left[v_{j+1}-v_{j}\right]+\sum_{j=1}^{k} p_{j}\left(1-e^{-g_{T}} F_{\tilde{X}}\left(p_{j}\right)\right) F_{\Gamma}^{\prime}\left(v_{j}\right)\left[v_{j+1}-v_{j}\right] \\
& \approx \sum_{j=0}^{L-k-2}\left(e^{-N_{0} v_{k+1} \theta^{j}} \frac{N_{0}\left(1+P_{c} v_{k+1} \theta^{j}\right)+P_{c}}{\left(1+P_{c} v_{k+1} \theta^{j}\right)^{2}}\right) c_{S U}[\theta-1] \\
& +\sum_{j=0}^{k-1}\left(1-e^{-g_{T}}+\frac{\theta^{j} e^{-g_{T}}}{\theta^{j}+c_{o} p_{1}}\right)\left(e^{-N_{0} v_{1} \theta^{j}} \frac{N_{0}\left(1+P_{c} v_{1} \theta^{j}\right)+P_{c}}{\left(1+P_{c} v_{1} \theta^{j}\right)^{2}}\right) c_{S U}[\theta-1] \\
\approx & c_{S U}\left(N_{0}+P_{c}\right)\left[e^{-g_{T}} E_{1}\left(N_{0} v_{1} \theta^{k}\right)-E_{1}\left(N_{0} v_{k+1} \theta^{L-k-1}\right)\right] \\
& +\left(1-e^{-g_{T}}\right)\left(N_{0}+P_{c}\right) c_{S U} E_{1}\left(N_{0} v_{1}\right) \\
& +\left[A_{2}\left(v_{k+1} \theta^{L-k-1}\right)-A_{2}\left(v_{k+1}\right)\right]+\left[A_{3}\left(v_{1} \theta^{k}\right)-A_{3}\left(v_{1}\right)\right]
\end{aligned}
$$




$$
\begin{aligned}
& \stackrel{(d)}{=} c_{S U}\left(N_{0}+P_{c}\right)\left[e^{-g_{T}} E_{1}\left(N_{0} v_{0} \theta^{k+1}\right)-E_{1}\left(N_{0} v_{k+1} \theta^{L-k-1}\right)\right] \\
& +\left(1-e^{-g_{T}}\right)\left(N_{0}+P_{c}\right) c_{S U} E_{1}\left(N_{0} v_{0} \theta\right) \\
& +\left[A_{2}\left(v_{k+1} \theta^{L-k-1}\right)-A_{2}\left(v_{k+1}\right)\right]+\left[A_{3}\left(v_{0} \theta^{k+1}\right)-A_{3}\left(v_{0} \theta\right)\right] \\
& =\mathcal{F}_{A T P C}(\theta, k)
\end{aligned}
$$

where

$$
\begin{aligned}
A_{2}(z) & =c_{S U} P_{c}\left(e^{\frac{N_{0}}{P_{c}}} E_{1}\left(N_{0} z+\frac{N_{0}}{P_{c}}\right)+\frac{e^{-N_{0} z}}{1+P_{c} z}\right) \\
A_{3}(z) & =\frac{c_{S U}}{P_{c} c_{o} c_{S U}-1}\left[\left(N_{0}-\frac{P_{c}}{P_{c} c_{o} c_{S U}-1}\right) e^{-g_{T}} e^{N_{0} c_{o} c_{S U}} E_{1}\left(N_{0}\left(z+c_{o} c_{S U}\right)\right)\right] \\
& +\frac{P_{c} c_{S U}}{P_{c} c_{o} c_{S U}-1}\left[\left(\frac{e^{-g_{T}+\left(1-e^{-g_{T}}\right)\left(P_{c} c_{o} c_{S U}-1\right)^{2}}}{P_{c} c_{o} c_{S U}-1}\right) e^{\frac{N_{0}}{P_{c}}} E_{1}\left(N_{0} z+\frac{N_{0}}{P_{c}}\right)-\frac{1-\left(1-e^{-g_{T}}\right) P_{c} c_{o} c_{S U}}{1+P_{c} z} e^{-N_{0} z}\right]
\end{aligned}
$$

and the third approximation in (D.23) is from the approximation by integration and the first equality in (D.23) is claimed by $v_{0}=v_{1} \theta$. 


\section{Bibliography}

[1] "Australian radiofrequency spectrum plan," Australian Communications and Media Authority, Tech. Rep., 2008. [Online]. Available: http://www.acma.gov.au/scripts/nc.dll?WEB/STANDARD/1001/pc=PC_2713

[2] "Spectrum policy task force," Federal Communications Commission (FCC), Rep. ET Docket no. 02-135, Tech. Rep., Nov. 2002.

[3] J. Mitola, "The software radio architecture," IEEE communication Magazine, vol. 33, no. 5, pp. 26-38, Nov. 1995.

[4] —, "Cognitive radio: An integrated agent architecture for software defined radio," Ph.D. dissertation, KTH, Stockholm, Sweden, Dec 2000.

[5] S. Haykin, "Cognitive radio: brain-empowered wireless communications," IEEE Journal on Selected Areas in Communications, vol. 23, no. 2, pp. 201-220, Feb 2005.

[6] A. Goldsmith, S. Jafar, I. Marić, and S. Srinivasa, "Breaking spectrum gridlock with cognitive radios: An information theoretic perspective," Proceedings of IEEE, vol. 97, no. 5, pp. 894 - 914, May 2009.

[7] N. Devroye, P. Mitran, and V. Tarokh, "Achievable rates in cognitive radio channels," IEEE Transactions on Information Theory, vol. 52, no. 5, pp. 18131827, May 2006.

[8] C. Shannon, "A mathematical theory of communication," Bell System Technical Journal, vol. 27, pp. 379-423, 623-656, 1948.

[9] J. Wolfowitz, Coding Theorems of Information Theory. Secaucus, NJ, USA: Springer-Verlag New York, Inc., 1964. 
[10] A. Goldsmith and P. Varaiya, "Capacity of fading channel with channel side information," IEEE Transactions on Wireless Communications, vol. 43, pp. 1986-1992, 1995.

[11] T. Cover and J. Thomas, Elements of Information Theory, 2nd ed. Hoboken, New Jersey.: John Wiley \& Sons, 2006.

[12] D. Tse and P. Viswanath, Fundamentals of Wireless Communication. Cambridge, U.K.: Cambridge Univ. Press, 2005.

[13] R. Knopp and P. Humblet, "Information capacity and power control in singlecell multiuser communications," in IEEE ICC, vol. 1, Seattle, USA, 18-22 June 1995, pp. 331-335.

[14] D. Tse and S. Hanly, "Multiaccess fading channels part I: Polymatroid structure, optimal resource allocation and throughput capacities," IEEE Transactions on Information Theory, vol. 44, no. 7, pp. 2796-2815, Nov. 1998.

[15] D. Tse, "Optimal power allocation over parallel gaussian broadcast channels," in IEEE ISIT, Ulm, Germany, 29 Jun-4 Jul 1997.

[16] L. Li and A. Goldsmith, "Capacity and optimal resource allocation for fading broadcast channels Part I: Ergodic capacity," IEEE Transactions on Information Theory, vol. 47, no. 3, pp. 1083-1101, March 2001.

[17] K. S. Gilhousen, I. M. Jacobs, R. Padovani, A. J. Viterbi, L. Weaver, and C. E. Wheatley, "On the capacity of a cellular cdma system," IEEE Transactions on Vehicular Technology, vol. 40, no. 2, pp. 303-312, May 1991.

[18] R. D. Yates, "A framework for uplink power control in cellular radio systems," IEEE Journal on Selected Areas in Communications, vol. 13, no. 7, pp. 13411347, Sept. 1995.

[19] S. Hanly and D. Tse, "Multiaccess fading channels part II: Delay-limited capacities," IEEE Transactions on Information Theory, vol. 44, no. 7, pp. 2816-2831, November 1998. 
[20] A. Goldsmith, Wireless Communications. Cambridge, U.K.: Cambridge Univ. Press, 2005.

[21] G. Caire, G. Taricco, and E. Biglieri, "Optimum power control over fading channels," IEEE Transactions on Information Theory, vol. 5, pp. 1468-1489, 1999.

[22] L. Li, N. Jindal, and A. Goldsmith, "Outage capacities and optimal power allocation for fading multiple-access channels," IEEE Transactions on Information Theory, vol. 4, no. 4, pp. 1326-1347, April 2005.

[23] L. Li and A. Goldsmith, "Capacity and optimal resource allocation for fading broadcast channels Part II: Outage capacity," IEEE Transactions on Information Theory, vol. 47, no. 3, pp. 1103-1127, 2001.

[24] N. Jindal, S. Vishwanath, and A. Goldsmith, "On the duality of gaussian multiple-access and broadcast channels," IEEE Transactions on Information Theory, vol. 50, no. 5, pp. 768-783, May 2004.

[25] J. Luo, R.Yates, and P. Spasojevic, "Service outage based power and rate allocation for parallel fading channels," IEEE Transactions on Information Theory, vol. 51, no. 7, pp. 2594-2611, July 2005.

[26] K. Chakraborty, S. S. Dey, and M. Franceschetti, "Service-outage-based power and rate control for poisson fading channels," IEEE Transactions on Information Theory, vol. 55, no. 5, pp. 2304-2318, May 2009.

[27] Y. Zeng, Y.-C. Liang, A. Hoang, and R. Zhang, "A review on spectrum sensing for cognitive radio: Challenges and solutions," EURASIP Journal on Advances in Signal Processing, vol. 2010, pp. 1-15, 2010.

[28] Y.-C. Liang, Y. Zeng, E. C. Y. Peh, and A. T. Hoang, "Sensing - throughput tradeoff for cognitive radio networks," IEEE Transactions on Wireless Communications, vol. 7, no. 4, pp. 1326-1337, April 2008. 
[29] O. Simeone, U. Spagnolini, and Y. Bar-Ness, "Stable throughput of cognitive radios with and without relaying capability," IEEE Transactions on Communications, vol. 55, no. 12, pp. 2351-2360, Dec 2007.

[30] Y. Pei, Y.-C. Liang, K. C. Teh, and K. H. Li, "How much time is needed for wideband spectrum sensing?" IEEE Transactions on Wireless Communications, vol. 8, no. 11, pp. 5466-5471, Nov 2009.

[31] S. I. Gel'fand and M. S. Pinsker, "Coding for channel with random parameters," Problem of Control and Information Theory, vol. 9, no. 1, pp. 19 -31, 1980.

[32] T. Han and K. Kobayashi, "A new achievable rate region for the interference channel," IEEE Transactions on Information Theory, vol. 27, no. 1, pp. 49-60, Jan 1981.

[33] M. Costa, "Writing on dirty paper," IEEE Transactions on Information Theory, vol. 29, no. 3, pp. 439-441, May 1983.

[34] H. Weingarten, Y. Steinberg, and S. Shamai(Shitz), "The capacity region of the gaussian MIMO broadcast channel," IEEE Transactions on Information Theory, vol. 52, no. 9, pp. 3936 - 3964, Sept. 2006.

[35] A. Jovičić and P. Viswanath, "Cognitive radio: An information-theoretic perspective," IEEE Transactions on Information Theory, vol. 55, no. 9, pp. 39453958, Sept. 2009.

[36] Q. Zhao and B. Sadler, "A survey of dynamice spectrum access," IEEE Signal Processing Magazine, vol. 24, pp. 79-89, 2007.

[37] M. Gastpar, "On capacity under receive and spatial spectrum-sharing constraints," IEEE Transactions on Information Theory, vol. 53, no. 2, pp. 471487, Feb. 2007.

[38] A. Ghasemi and E. S. Sousa, "Fundamental limits of spectrum-sharing in fading environments," IEEE Transactions on Wireless Communications, vol. 6, no. 2, pp. 649-658, Feb 2007. 
[39] X. Kang, Y.-C. Liang, A. Nallanathan, H. Garg, and R. Zhang, "Optimal power allocation for fading channels in cognitive radio networks: Ergodic capacity and outage capacity," IEEE Transactions on Wireless Communications, vol. 8, no. 2, pp. 940-950, Feb. 2009.

[40] X. Kang, Y.-C. Liang, A. Nallanathan, H. Garg, and L. Zhang, "Sensing-based spectrum sharing in cognitive raio networks," IEEE Transactions on Vehicular Technology, vol. 58, no. 8, pp. 4649-4654, Oct. 2009.

[41] L. Musavian and S. Aïssa, "Quality-of-service based power allocation in spectrum-sharing channels," in IEEE GLOBECOM, New Orleans, LO, 30 Nov.4 Dec. 2008 , pp. $1-5$.

[42] D. Wu and R. Negi, "Effective capacity : A wireless link model for support of Quality of Service," IEEE Transactions on Wireless Communications, vol. 2, no. 4, pp. 630-643, July 2003.

[43] R. Zhang, "Optimal power control over fading cognitive radio channels by exploiting primary user CSI," in IEEE GLOBECOM, New Orleans, USA, Nov. 2008, pp. 1-5.

[44] X. Kang, R. Zhang, Y.-C. Liang, and H. Garg, "Optimal power allocation strategies for fading cognitive radio channels with primary user outage constraint," IEEE Journal on Selected Areas in Communications, vol. 29, no. 2, pp. $374-383$, Feb. 2011.

[45] K. Eswaran, M. Gastpar, and K. Ramchandran, "Bits through arqs: Spectrum sharing with a primary packet system," in IEEE International Symposium on Information Theory (ISIT), Nice, France, June 2007, pp. 2171-2175.

[46] G. Ganesan and Y. Li, "Cooperative spectrum sensing in cognitive radio networks," in IEEE International Symposium on New Front. Dynamic Spectrum Access Networks (DySPAN), Atlanta, GA, Nov. 2005, pp. 137-143. 
[47] V. Chakravarthy, A. Nunez, J. Stephens, A. Shaw, and M. Temple, "TDCS, OFDM, and MC-CDMA : A brief tutorial," IEEE Communications Magazine, vol. 43, no. 9, pp. S11 - S16, Sept. 2005.

[48] T. Weiss and F. Jondral, "Spectrum pooling: an innovative strategy for the enhancement of spectrum efficiency," IEEE Communications Magazine, vol. 42, no. 3, pp. S8 - S14, Mar 2004.

[49] G. Bansal, J. Hossain, and V. Bhargava, "Optimal and suboptimal power allocation schemes for OFDM-based cognitive radio systems," IEEE Transactions on Wireless Communications, vol. 7, no. 11, pp. 4710-4718, Nov. 2008.

[50] P. Wang, M. Zhao, L. Xiao, S. Zhou, and J. Wang, "Power allocation in OFDMbased cognitive radio systems," in IEEE GLOBECOM, Washington, DC, 26 -30 Nov. 2007, pp. 4061-4065.

[51] X. Kang, H. Garg, Y.-C. Liang, and R. Zhang, "Optimal power allocation for OFDM-based cognitive radio with new primary transmission protection criteria," IEEE Transactions on Wireless Communications, vol. 9, no. 6, pp. 2066$2075,2010$.

[52] R. Zhang, S. Cui, and Y.-C. Liang, "On ergodic sum capacity of fading cognitive multiple-access and broadcast channels," IEEE Transactions on Information Theory, vol. 55, no. 11, pp. 5161-5178, Nov. 2009.

[53] X. Kang, Y.-C. Liang, and H. Garg, "Fading cognitive multiple access channels : Outage capacity regions and optimal power allocation," IEEE Transactions on Wireless Communications, vol. 9, no. 7, pp. 2382 - 2391, July 2010.

[54] M. Sharif and B. Hassibi, "On the capacity of MIMO broadcast channels with partial side information," IEEE Transactions on Information Theory, vol. 51, no. 2, pp. 506 - 522, Feb 2005.

[55] C. Cornell, "Engineering in seismic risk analysis," Bulletin of the Seismological Society of America, vol. 58, no. 5, pp. 1583-1606, Oct. 1968. 
[56] E. of tail-related risk measures for heteroscedastic financial time series: an extreme value approach, "A. j. mcneil and rudiger frey," Journal of Empirical Finance, vol. 3, no. 7, p. 271300, Nov. 2000.

[57] R. Zhang and Y.-C. Liang, "Investigation on multiuser diversity in spectrum sharing based cognitive radio networks," IEEE Communications Letters, vol. 14, no. 2, February 2010.

[58] E. Nekouei, H. Inaltekin, and S. Dey, "Throughput scaling in cognitive multiple access with power and interference constraints," IEEE Transactions on Signal Processing, vol. 60, no. 2, pp. 927-946, Feb 2011.

[59] M. A. Khojastepour, G. Yue, X. Wang, and M. Madihian, "Optimal power control in MIMO systems with quantized feedback," IEEE Transactions on Wireless Communications, vol. 7, no. 12, pp. 4859-4866, December 2008.

[60] Y. He and S. Dey, "Power allocation in spectrum sharing cognitive radio networks with quantized channel information," IEEE Transactions on Communications, vol. 59, no. 6, pp. 1644-1656, June 2011.

[61] — , "Power allocation for outage minimization in cognitive radio network with limited-feedback," IEEE Transactions on Communications, 2011, submitted.

[62] — _ "Efficient algorithms for outage minimization in parallel fading channels with limited feedback," in 47th Annual Allerton Conference on Communication, Control, and Computing, Monticello, IL, 30 Sept.-2 Oct. 2009, pp. 762 - 770.

[63] — - "Throughput maximization in cognitive radio under peak interference constraints with limited feedback," IEEE Transactions on Vehicular Technology, vol. 9, pp. 1287-1305, Mar 2012.

[64] A. Narula, M. Lopez, M. D. Trott, and G. W. Wornell, "Efficient use of side information in multiple-antenna data transmission over fading channels," IEEE Journal on Selected Areas in Communications, vol. 16, no. 8, pp. 1423-1436, 1998. 
[65] D. J. Love and R. W. Heath, "Grassmannian beamforming for multipleinput multiple-output wireless systems," IEEE Transactions on Information Theory, vol. 49, no. 10, pp. 2735-2747, Oct. 2003.

[66] P. Xia and G. B. Giannakis, "Design and analysis of transmitbeamforming based on limited-rate feedback," IEEE Transactions on Signal Processing, vol. 54, no. 5, pp. 1853-1863, May 2006.

[67] T. T. Kim and M. Skoglund, "Diversity-multiplexing tradeoff in MIMO channels with partial CSIT," IEEE Transactions on Information Theory, vol. 53, no. 8, pp. 2743-2759, Aug. 2007.

[68] S. Bhashyam, A. Sabharwal, and B. Aazhang, "Feedback gain in multiple antenna systems," IEEE Transactions on Communications, vol. 50, no. 5, pp. 785-798, May 2002.

[69] A. Khoshnevis and A. Sabharwal, "On the asymptotic performance of multiple antenna channels with quantized feedback," IEEE Transactions on Wireless Communications, vol. 7, no. 10, pp. 3869-3877, Oct. 2008.

[70] B. Khoshnevis and W. Yu, "Joint power control and beamforming codebook design for MISO channels with limited feedback," in IEEE GLOBECOM, Honolulu, Hawaii, 30 Nov.-4 Dec. 2009.

[71] K. Huang and R. Zhang, "Cooperative feedback for multiantenna cognitive radio networks," IEEE Transactions on Signal Processing, vol. 59, no. 2, pp. 747-758, Feb 2011.

[72] J. Jang and K. B. Lee, "Transmit power adaptation for multiuser ofdm systems," IEEE Journal on Selected Areas in Communications, vol. 21, no. 2, pp. $171-178$, Feb. 2003.

[73] X. Kang, R. Zhang, Y.-C. Liang, and H. K. Garg, "Optimal power allocation for cognitive radio under primary user's outage loss constraint," in IEEE International Conference on Communications, Dresden, Germany, June 14-18, 2009, pp. $1-5$. 
[74] — _ "On outage capacity of secondary users in fading cognitive radio networks with primary users outage constraint," in IEEE GLOBECOM, Hawaii, USA, Nov 2009, pp. 1-5.

[75] L. Musavian, S. Aïssa, and S. Lambotharan, "Adaptive modulation in spectrum-sharing channels under delay quality-of-service constraints," IEEE Transactions on Vehicular Technology, vol. 60, no. 3, pp. 901 - 911, March 2011.

[76] A. Limmanee, S. Dey, and J. Evans, "Service-outage capacity maximization in cognitive radio," in Proceedings of the IEEE International Conference on Communications (ICC), Kyoto, Japan, June 2011, pp. 1-6.

[77] K. Eswaran, M. Gastpar, and K. Ramchandran, "Bits through ARQs: Spectrum sharing with a primary packet system," in IEEE International Symposium on Information Theory, June 2007, pp. $2171-2175$.

[78] K. Ahn and R. W. Heath, "Performance analysis of maximum ratio combining with imperfect channel estimation in the presence of cochannel interferences," IEEE Transactions on Wireless Communications, vol. 8, no. 8, pp. 1080 - 1085, March 2009.

[79] T. W. Ban, W. Choi, B. C. Jung, and D. K. Sung, "Multi-user diversity in a spectrum sharing system," IEEE Transactions on Wireless Communications, vol. 8, no. 8, pp. 102-106, January 2009.

[80] H. Wang, J. Lee, S. Kim, and D. Hong, "Capacity of secondary users exploiting multispectrum and multiuser diversity in spectrum-sharing environments," IEEE Transactions on Vehicular Technology, vol. 59, no. 2, pp. 1030-1036, February 2010.

[81] E. Nekouei, H. Inaltekin, and S. Dey, "Throughput scaling in cognitive multiple access networks with power and interference constraints," in IEEE International Conference on Communications, Kyoto, Japan, 5 - 9 June 2011, accepted. 
[82] M. R. Leadbutter, G. Lindgren, and H. Rootzen, Extremes and Related Properties of Random Sequences and Processes. Springer-Verlag, New York, 1983.

[83] H. A. David and H. N. Nagaraja, Order Statistics. John Wiley and Sons, New Jersey, 2003.

[84] L. Zhang, Y. Xin, Y.-C. Liang, and H. V. Poor, "Cognitive multiple access channels: optimal power allocation for weighted sum rate maximization," IEEE Transactions on Communications, vol. 57, no. 9, pp. 2754-2762, Sep. 2009.

[85] W. Wang, Q. Lu, K. G. Shin, and T. Peng, "Geometry-based optimal power control of fading multiple acccess channels for maximum sum-rate in cognitive radio networks," IEEE Transactions on Wireless Communications, vol. 9, no. 6, pp. 1843-1848, June 2010.

[86] M. Médard, "The effect upon channel capacity in wireless communications of perfect and imperfect knowledge of the channel," IEEE Transactions on Information Theory, vol. 46, no. 3, pp. 933-945, May 2000.

[87] L. Musavian and S. Aïssa, "Fundamental capacity limits of cognitive radio in fading environments with imperfect channel information," IEEE Transactions on Commuications, vol. 57, no. 11, pp. 3472 - 3480, Nov. 2009.

[88] H. Suraweera, P. Smith, and M. Shafi, "Capacity limits and performance analysis of cognitive radio with imperfect channel knowledge," IEEE Transactions on Vehicular Technology, vol. 59, no. 4, pp. 1811-1822, May 2010.

[89] J. C. Spall, "Implementation of the simultaneous perturbation algorithm for stochastic optimization," IEEE Transactions on Aerospace and Electronic Systems, vol. 34, no. 3, pp. 817-823, July 1998.

[90] S. Boyd and L. Vandenberghe, Convex Optimization. Cambridge, U.K.: Cambridge Univ. Press, 2004. 


\section{University Library}

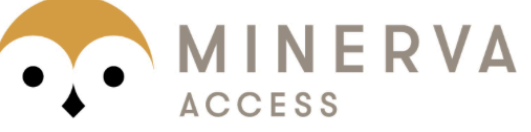

A gateway to Melbourne's research publications

Minerva Access is the Institutional Repository of The University of Melbourne

Author/s:

LIMMANEE, ATHIPAT

Title:

Resource allocation in cognitive radio networks

Date:

2012

Citation:

Limmanee, A. (2012). Resource allocation in cognitive radio networks. PhD thesis,

Department of Electrical and Electronic Engineering, The University of Melbourne.

Persistent Link:

http://hdl.handle.net/11343/38071

Terms and Conditions:

Terms and Conditions: Copyright in works deposited in Minerva Access is retained by the copyright owner. The work may not be altered without permission from the copyright owner. Readers may only download, print and save electronic copies of whole works for their own personal non-commercial use. Any use that exceeds these limits requires permission from the copyright owner. Attribution is essential when quoting or paraphrasing from these works. 